\title{
Calibration of the ATLAS Forward Calorimeter and $a$ \\ Study of the Top Quark Production Cross-section Measurement in the Dilepton Channel
}

\author{
by
}

\section{Louise Heelan}

\author{
A thesis submitted to the \\ Faculty of Graduate Studies and Research \\ in partial fulfillment of the requirements \\ for the degree of \\ Doctor of Philosophy \\ Ottawa-Carleton Institute for Physics \\ Department of Physics \\ Carleton University \\ Ottawa, Canada \\ December, 2010
}

(C)Copyright 2010 Louise Heelan 
Library and Archives

Canada

Published Heritage

Branch

395 Wellington Street

Ottawa ON K1A ON4

Canada
Bibliothèque et

Archives Canada

Direction du

Patrimoine de l'édition

395 , rue Wellington

Ottawa ON K1A ON4

Canada
Your file Votre reférence

ISBN: 978-0-494-79632-0

Our file Notre référence

ISBN: $978-0-494-79632-0$
NOTICE:

The author has granted a nonexclusive license allowing Library and Archives Canada to reproduce, publish, archive, preserve, conserve, communicate to the public by telecommunication or on the Internet, loan, distribute and sell theses worldwide, for commercial or noncommercial purposes, in microform, paper, electronic and/or any other formats.

The author retains copyright ownership and moral rights in this thesis. Neither the thesis nor substantial extracts from it may be printed or otherwise reproduced without the author's permission.

\section{AVIS:}

L'auteur a accordé une licence non exclusive permettant à la Bibliothèque et Archives Canada de reproduire, publier, archiver, sauvegarder, conserver, transmettre au public par télécommunication ou par l'Internet, prêter, distribuer et vendre des thèses partout dans le monde, à des fins commerciales ou autres, sur support microforme, papier, électronique et/ou autres formats.

L'auteur conserve la propriété du droit d'auteur et des droits moraux qui protège cette thèse. $\mathrm{Ni}$ la thèse ni des extraits substantiels de celle-ci ne doivent être imprimés ou autrement reproduits sans son autorisation.
In compliance with the Canadian Privacy Act some supporting forms may have been removed from this thesis.

While these forms may be included in the document page count, their removal does not represent any loss of content from the thesis.
Conformément à la loi canadienne sur la protection de la vie privée, quelques formulaires secondaires ont été enlevés de cette thèse.

Bien que ces formulaires aient inclus dans la pagination, il n'y aura aucun contenu manquant. 


\section{Abstract}

Over the last 20 years the ATLAS detector has gone from an idea to an operational detector. In that time thousands of people have contributed by way of research, development, installation, calibration, maintenance, and analysis. This thesis describes my contribution to that effort.

A test beam took place in 2003 with the ATLAS forward calorimeter. With this data the electromagnetic response of FCall was confirmed to be within $\pm 1.5 \%$ from unity in the range of energies from $10 \mathrm{GeV}$ to $200 \mathrm{GeV}$. The intrinsic energy resolution of the FCal was found to be $(27.0 \pm 1.0) \% / \sqrt{E} \oplus(3.5 \pm 0.1) \%$ for electrons and $(86.5 \pm 3.6) \% / \sqrt{E} \oplus(7.2 \pm 1.1) \%$ for hadrons (with a simple hadronic weighting scheme applied). Variations in parameters of the topological clustering algorithm were studied, and a suggestion is made that the energy cut parameter of the algorithm be increased from the default $500 \mathrm{MeV}$ to about $1 \mathrm{GeV}$. A study with electrons and hadrons confirms that using calorimeter shower shape information, a likelihood can be built and used to help identify these two types of showers. Monte Carlo and data comparisons find that the electromagnetic energy scale of the Monte Carlo agrees with the data within $\pm 2 \%$ for $10 \mathrm{GeV}$ to $200 \mathrm{GeV}$ hadrons. However, the Monte Carlo showers tend to be more dense, more narrow, and shorter than the data for high energy hadrons (with energies $\geq 60 \mathrm{GeV}$ ). Using the test beam data and Monte Carlo, a study was made on the characteristics of energy deposition at large values of 
$|\eta|$ near the LHC beam pipe. A correction for the energy loss is suggested in order to improve the response by $13 \%$ and the energy resolution by $12 \%$ for hadrons striking the FCal front face at $4.3<|\eta|<4.9$.

This thesis also examines the expected performance of the ATLAS detector to a $t \bar{t}$ cross-section measurement using the dilepton decay channel, with either electrons or muons in the final state along with jets and missing transverse energy. The choice of generator and parton shower algorithm used for Monte Carlo generation does affect the final event kinematics, with differences between next-to-leading order and leading order generators having the largest discrepancy. If a transverse momentum cut of $20 \mathrm{GeV}$ is applied to the reconstructed electrons and muons, the trigger efficiency for single electrons is found to be $98.7 \%$, and for single muons $82.1 \%$. Using the single lepton triggers the signal-to-background ratio was found to be 2.6 for the ee channel, 2.7 for the $\mu \mu$ channel, and 5.3 for the $e \mu$ channel; similar results were found with the dilepton triggers, motivating the desire of having these triggers in the ATLAS trigger menu. A measurement of the $t \bar{t}$ cross-section using the dilepton channels will be possible with $200 \mathrm{pb}^{-1}$ of data with $\sqrt{s(p p)}=7 \mathrm{TeV}$, but in the early stages of ATLAS data taking this measurement will be limited by the luminosity uncertainty. 


\section{Acknowledgements}

Going back five years (and maybe more...) I would like to express my thanks to Richard Hemingway and the other members of the Carleton University SNO collaboration. That experience at Carleton working on SNO was so life changing and inspiring that I willingfully signed myself up for another five years as a Ph.D. student on the ATLAS experiment with Gerald Oakham.

They say your graduate supervisor will make or break your experience as a graduate student. And I have absolutely had a fantastic experience as a Ph.D. student at Carleton. I could not have asked for more. Gerald - I would like to express my deepest gratitude for your support, wisdom, and encouragement. You gave me so many great opportunities to take on tasks and responsibilities that allowed me to grow as a researcher and individual. Aside from supervising me, over the years I have watched you handle with grace the taxing roles you have played as supervisor, graduate chair, head of our group. I have silently watched and learned from your patience and leadership, skills that I hope will follow me into the future years of my career.

I would like to thank the members of the forward calorimeter test beam with whom I worked. The people from the University of Arizona: John Rutherfoord, and Alexandre Savine for their attention to detail and push for perfection, and to Walter Lampl for help with test beam software and databases. The people from 
the University of Toronto: Peter Krieger and Peter Thompson, you both helped my analysis by providing steady support and encouragement. And the people from within Carleton University: J.P. Archambault, Dag Gillberg, Mohsen Khakzad, Tom McCarthy, and Malachi Schram.

I would sincerely like to thank the entire department at Carleton University. Each member has helped me grow into the person I am today, professionally and personally. In some way all of you of have touched my life. A big thank you to Heather Logan for carefully reviewing the first chapter of this thesis. I would like to thank the people who act behind the scenes to support the work being done, these people include the secretaries, laboratory and computing staff. I would especially like to acknowledge Wade, Yun-Ha, and Bill. Thank you to the past and present members of the Carleton ATLAS group. To the current and future inhabitants of HP1154, never give up those heaters ... never!

To Olivier, thank you. You had a profound influence on my life here at Carleton, and I will never forget or regret the laughs and tears we shed. Thank you for all the life's lessons, from culinary adventures, to trips abroad and local, to your computing tips and tricks, to your help with our courses. You have always inspired me to be the best. I will always have a special place in my heart for you.

Thank you to my Genf family (you know who you are); you all maintain an incredible balance between work and play, thank you for keeping me sane through all the years (although I know y'all don't think I'm that sane).

I would like to thank my closest friends Laura and Kristi. You are both strong and independent women who have shown me that as a women you can rise to the top as a professional, wife, and mother. Thank you for the years of support and encouragement.

I would like to thank Eddie for giving me the support and push needed to finish 
this work. Thank you for being my ear and listening as I talked through my analysis problems. At this point you probably know the FCal just as well as me! As I end this work and put the final ink on the pages, I can't wait to move forward with you and see what the next chapter of our lives will hold.

Finally, I would like to thank my family. To my Dad, thank you for your love and support throughout all of the years; did you ever imagine that little girl from Kilcoole would end up here? To my brother David, you have such focus and you constantly surprise me with your hidden talents, you need to find your opportunity as I did here, let go and trust in the people around you, they will be there for you. To my sister Pauline, you amaze and inspire me and I could not imagine my life without you. Thank you for fighting to survive, and fighting for others to survive. You are a hero to me and so many others. I have to thank Tim, my step father, for being a good listener and observer, somehow you can read my emotions like a book and support whatever emotions emerge from those pages with an open heart and mind. To my Mom, if there is anyone to whom this great achievement is dedicated to, it is you. You are my greatest supporter, my greatest friend. You have always reminded me what a rare and unique opportunity this has been. There is no way this thesis would exist without you, and that is the absolute truth.

I have been so fortunate to have so much love in my life, and to those people (named and unnamed above) I can never thank you enough for sharing this journey. Thank you for letting me into your world and sharing your joys and sorrows, as I do here, welcoming you into my world of physics, something that has brought me joy, happiness, pain, and pride. 


\section{Contents}

$\begin{array}{lll}\text { Abstract } & \text { ii }\end{array}$

Acknowledgments $\quad$ iv

List of Tables $\quad$ xi

List of Figures $\quad$ xiv

List of Acronyms xxi

1 The Standard Model of Particle Physics 1

1.1 Quarks and Leptons . . . . . . . . . . . . . . . . 2

1.2 Fundamental Interactions $\ldots \ldots \ldots \ldots \ldots$

1.2.1 Electromagnetism . . . . . . . . . . . . . . . . 4

1.2 .2 Strong Interaction $\ldots \ldots \ldots \ldots \ldots \ldots$

1.2.3 (Electro)Weak Interaction and the Higgs Mechanism . . . . 6

1.3 Summary . . . . . . . . . . . . . . . . . . . 11

1.4 A Look Ahead . . . . . . . . . . . . . . . . . . . . . 12

2 The Large Hadron Collider and the ATLAS Detector 14

2.1 The Large Hadron Collider . . . . . . . . . . . . . . . . 15

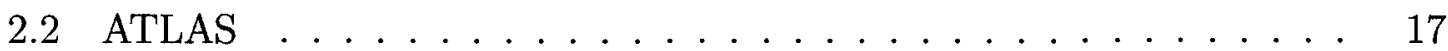

2.2 .1 Coordinate System . . . . . . . . . . . . . . . 18

2.2 .2 Magnet System . . . . . . . . . . . . . . . . . . . 19

2.2 .3 Inner Detector . . . . . . . . . . . . . . . . . . . . 21

2.2 .4 Calorimeters . . . . . . . . . . . . . . . . . 23

2.2 .5 Muon Spectrometer . . . . . . . . . . . . . . . 28 
2.3 Software for Analysis . . . . . . . . . . . . . . . 30

3 Calibration of the ATLAS Forward Calorimeter 32

3.1 Particle Interactions in Matter . . . . . . . . . . . . . . . . 33

3.1.1 Massive Particle Interactions in Matter . . . . . . . . . . . 33

3.1.2 Electron Interactions in Matter . . . . . . . . . . . 35

3.1.3 Photon Interactions in Matter . . . . . . . . . . . . . 37

3.2 Particle Showers . . . . . . . . . . . . . . . . . . . 39

3.2 .1 Electromagnetic Showers . . . . . . . . . . . . . . . 39

3.2 .2 Hadronic Showers . . . . . . . . . . . . . . . . . 43

3.3 Calorimeters for Particle Detection . . . . . . . . . . . . . 45

3.4 The ATLAS Forward Calorimeter . . . . . . . . . . . . . . 48

3.4 .1 Electronics Chain . . . . . . . . . . . . . . . . 52

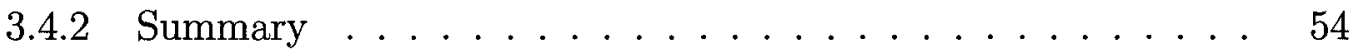

3.5 Test Beam Introduction $\ldots \ldots \ldots \ldots \ldots \ldots$

3.5.1 Beam Line Detectors and Associated Data Quality Cuts . . . 58

3.5 .2 Event Timing . . . . . . . . . . . . . . . 62

3.5.3 Summary of Runs Taken . . . . . . . . . . . . . . . 64

3.6 Reconstruction of the Electronic Signal . . . . . . . . . . . . 65

3.6.1 Optimal Filtering Technique . . . . . . . . . . . . 67

3.6 .2 Pedestal and Noise . . . . . . . . . . . . . . . . 69

3.6 .3 Summary . . . . . . . . . . . . . . . . . 72

3.7 Channel Clustering . . . . . . . . . . . . . . . . . . 75

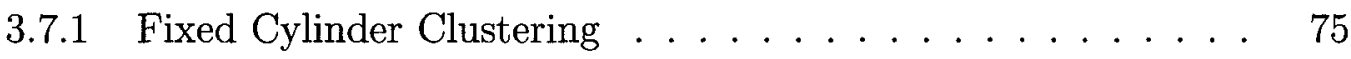

3.7 .2 Topological Clustering . . . . . . . . . . . . . . . . 81

3.8 Intrinsic Response of the Forward Calorimeter . . . . . . . . . . 85

3.8 .1 Electron Analysis . . . . . . . . . . . . . . . 85

3.8 .2 Hadron Analysis . . . . . . . . . . . . . . . . . . 96

3.8.3 Correction to Previous Published Results . . . . . . . . . . . 102

3.9 Investigation of the Topological Clustering Algorithm . . . . . . . . 104

3.9.1 Comparison of Cylinder and Topological Clustering . . . . . 104

3.9.2 Topological Clustering Shower Shape Variables . . . . . . . . 111

3.9.3 Topological Cluster Splitting Algorithm . . . . . . . . . . 116

3.9.4 Summary of Topological Clustering Results . . . . . . . 123 
3.10 Monte Carlo Method . . . . . . . . . . . . . . . . . . . . . . 124

3.10.1 Data and Monte Carlo Comparisons . . . . . . . . . . 129

3.10.2 Data and Monte Carlo Topological Cluster Shower Shapes . . 134

3.11 Response of the Forward Calorimeter at High $|\eta| \ldots \ldots$. . . . . . 140

3.11.1 Cluster Correction for Inner Edge Energy Loss . . . . . . . . 147

3.11.2 Data and Monte Carlo Comparisons of Energy Deposition . . 150

3.11.3 Data and Monte Carlo Comparisons of Cluster Multiplicities . 155

3.11 .4 Summary . . . . . . . . . . . . . . . 157

3.12 Summary of the Forward Calorimeter Test Beam Analysis . . . . 158

4 Determination of the Top Quark Pair Cross-section in ATLAS 160

4.1 The Role of the Top Quark in the Standard Model . . . . . . . . . 161

4.1 .1 Top Quark Production . . . . . . . . . . . . . . . . . . . 163

4.1 .2 Top Quark Decays . . . . . . . . . . . . . . . . . . . . 165

4.1.3 Current Status of Experimental Results . . . . . . . . . 167

4.2 Monte Carlo Data Sets . . . . . . . . . . . . . . . . . . . 168

4.2 .1 Generators . . . . . . . . . . . . . . . . . . . 168

4.2 .2 Signal Samples . . . . . . . . . . . . . . . . . . 171

4.2 .3 Background Samples . . . . . . . . . . . . . . . . . 179

4.3 Introduction to the Experimental Calculation of the Cross-section . . 180

4.4 Reconstructed Object Identification . . . . . . . . . . . . . . . 180

4.4 .1 Tau Leptons . . . . . . . . . . . . . . . . . . . . . . . . 182

4.4 .2 Electrons . . . . . . . . . . . . . . . . . . . . 182

4.4 .3 Muons . . . . . . . . . . . . . . . . . . . 188

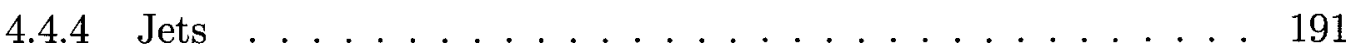

4.4 .5 Object Overlap Removal . . . . . . . . . . . . . . . . 195

4.4 .6 Missing Transverse Energy . . . . . . . . . . . . . . . . 197

4.4.7 Overlap Removal and Missing Transverse Energy . . . . . . 199

4.5 The ATLAS Trigger . . . . . . . . . . . . . . . 200

4.5 .1 Naming Convention . . . . . . . . . . . . . . . . . . 202

4.5.2 Trigger Menu and Trigger Rates . . . . . . . . . . . . . 202

4.5 .3 Electron Triggers . . . . . . . . . . . . . . . . . . . 203

4.5 .4 Muon Triggers. . . . . . . . . . . . . . . . . 205

4.5 .5 Trigger Efficiencies . . . . . . . . . . . . . . 208 
4.5.6 Object Trigger Efficiencies: Single Lepton Triggers _... . . 211

4.5.7 Object Trigger Efficiencies: Dilepton Triggers . . . . . . . . 217

4.5 .8 Object Trigger Efficiencies Summary . . . . . . . . . . . . 220

4.6 Event Selection and Expected Number of Signal and Background Events220

4.7 Cross-section Calculation . . . . . . . . . . . . . . . . . . . . . . . 229

4.7 .1 Reconstruction Efficiency . . . . . . . . . . . . . 230

4.7 .2 Trigger Efficiency . . . . . . . . . . . . . . . . . . 232

4.7.3 Luminosity and Associated Systematic Uncertainty . . . . 236

4.7.4 Variation with Monte Carlo Generator . . . . . . . . . 241

4.7 .5 Summary of Uncertainties . . . . . . . . . . . . . . . 242

4.7.6 Comparison of the Cross-section Calculation . . . . . . . . 243

4.7.7 Summary and Outlook . . . . . . . . . . . . . . . . . 244

5 Conclusion $\quad 246$

A Monte Carlo Data Set Information for Top Physics 247

B Efficiency Uncertainty $\quad 252$

B.1 The Standard Methods . . . . . . . . . . . . . . . . . . . . 252

B.1.1 Binomial Statistics . . . . . . . . . . . . . . . 252

B.1.2 Poisson Statistics . . . . . . . . . . . . . . . . . . 253

B.1.3 Bayesian Statistics . . . . . . . . . . . . . . . . . 254

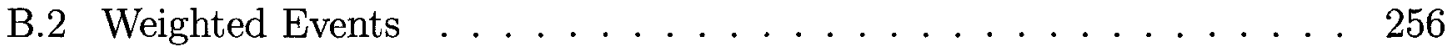

B.3 Example . . . . . . . . . . . . . . . . . . . . . 257

$\begin{array}{ll}\text { References } & 259\end{array}$ 


\section{List of Tables}

1.1 Charge, mass, and spin of the leptons. . . . . . . . . . . 2

1.2 Charge, mass, and spin of the quarks. . . . . . . . . . . 3

1.3 Properties of interactions and associated carrier particles. . . . . . . 12

2.1 Parameters of the Large Hadron Collider. . . . . . . . . . . 16

3.1 Definition of the variables in the Bethe-Bloch equation for energy loss of massive charged particles in matter. . . . . . . . . . . 34

3.2 Summary of the selected properties of the forward calorimeter. . . . . 54

3.3 Test beam impact points, and associated beam particle types and en-

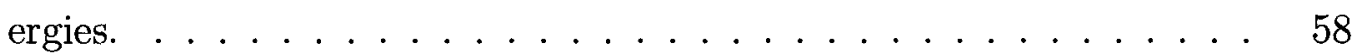

3.4 Alignment of the forward calorimeter (using cluster center coordinates) and the beam projection chambers (using track projections). . . . . 81

3.5 Total number of events after all event selection cuts for the $4 \mathrm{~L}$ electron data analysis. . . . . . . . . . . . . . . .

3.6 Mean and standard deviation of the electron energy distributions in position $4 \mathrm{~L} . \ldots \ldots \ldots \ldots \ldots \ldots \ldots \ldots$

3.7 Mean reconstructed energy and uncertainty for electron versus positron data. . . . . . . . . . . . . . . . . . .

3.8 Double Gaussian fit results to the electon data when clustered with a $120 \mathrm{~mm}$ and $160 \mathrm{~mm}$ radial cylinder. . . . . . . . . . . . . . . 92

3.9 Total number of events after all event selection cuts for the $4 \mathrm{~L}$ hadron

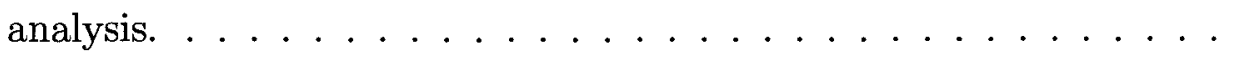

3.10 Mean and standard deviation of the hadron energy distributions, after the application of hadronic flat weights. . . . . . . . . . . 100 
3.11 Noise-subtracted energy resolution fit results for the stochastic and constant terms, for the default cylinder clustering and different configurations of the topological clustering for electrons in position $4 \mathrm{~L} . .$.

3.12 Fit values for the slope and intercept for the electron linearity data clustered with five topological clustering configurations of the seed, neighbour, perimeter parameters. . . . . . . . . . . . . . . 107

3.13 Noise-subtracted energy resolution fit results for the stochastic and constant terms, for the default cylinder clustering and different configurations of the topological clustering for hadrons in position $4 \mathrm{~L}$. . . .

3.14 Total number of events after all event selection cuts for the inner edge hadron analysis. . . . . . . . . . . . . . . . .

3.15 Fraction of total clustered energy in each FCal module, average number of clusters per event, and fraction of energy in the maximum energy cluster for hadrons in positions $1,2,3$, and $4 \mathrm{~L} \ldots \ldots \ldots$. . . . . . 142

4.1 The theoretical cross-sections for $t \bar{t}$ production at the Tevatron ( $p \bar{p}$ $\sqrt{s}=1.96 \mathrm{TeV})$ and the LHC $(p p \sqrt{s}=7 \mathrm{TeV}, 10 \mathrm{TeV}, 14 \mathrm{TeV}) . \ldots 166$

4.2 Branching ratios for $t \bar{t}$ decay modes. . . . . . . . . . . . . 167

4.3 Top quark Monte Carlo signal samples used in the analysis, including those used for systematic studies. . . . . . . . . . . . . . . . . . 172

4.4 Background samples considered for the $t \bar{t}$ cross-section analysis. . . . 180

4.5 Reconstructed electron selection criteria. . . . . . . . . . . . . 186

4.6 Reconstructed electron efficiency and purity for Loose, Medium, Medium Isolated, and Tight electrons (including detector acceptance) using $t \bar{t}$ dilepton events. . . . . . . . . . . . . . . . . . 188

4.7 Reconstructed muon selection criteria. . . . . . . . . . . . . . . 191

4.8 Average number of reconstructed jets per event and average $p_{\mathrm{T}}$, for six different jet reconstruction methods. . . . . . . . . . . . . . . . 194

4.9 Reconstructed jet selection criteria. . . . . . . . . . . . . . . . 195

4.10 Jet reconstruction efficiency and purity for the AntiKt4 and Cone4 algorithms. . . . . . . . . . . . . . . . . . 196

4.11 Selected L1, L2, and EF trigger items from the trigger menu. . . . . . 203

4.12 Trigger efficiency for electrons in selected electron-type triggers, and muons in muon-type triggers, in the plateau region of the trigger turnon curves. . . . . . . . . . . . . . . . . . . . . . . . 214 
4.13 Event selection criteria for the $t \bar{t}$ dilepton $e e, \mu \mu$, and $e \mu$ channels. . .

4.14 Total number of Monte Carlo events used for the signal and each of the backgrounds (before scaling to $200 \mathrm{pb}^{-1}$ at $\sqrt{s}=7 \mathrm{TeV}$ ). . . .

4.15 Signal and background number of events after the ee event selection for $200 \mathrm{pb}^{-1}$ at $7 \mathrm{TeV} \ldots \ldots \ldots \ldots \ldots \ldots$. . . . . . . . . 226

4.16 Signal and background number of events after the $\mu \mu$ event selection for $200 \mathrm{pb}^{-1}$ at $7 \mathrm{TeV} \ldots \ldots \ldots \ldots \ldots \ldots \ldots$ 228

4.17 Signal and background number of events after the $e \mu$ event selection for $200 \mathrm{pb}^{-1}$ at $7 \mathrm{TeV} \ldots \ldots \ldots \ldots \ldots \ldots$

4.18 Reconstruction efficiency with respect to the true dilepton channel for the $e e, \mu \mu$, and $e \mu$ channels. . . . . . . . . . . . . . . 231

4.19 Variations in the in the $t \bar{t}$ cross-section measurement by changing the jet energy 4 -vector by $\pm 5 \%$ and $\pm 10 \% \ldots \ldots \ldots \ldots 233$

4.20 Trigger efficiencies for the $e e, \mu \mu$, and $e \mu$ channels. . . . . . . . . 235

4.21 Break down of the systematic uncertainties to the top pair cross-section measurement. . . . . . . . . . . . . . . . . . . . 242

A.1 Additional Monte Carlo information for the $t \bar{t}$ samples. . . . . . . . 247

A.2 Additional Monte Carlo information for the $Z \rightarrow l l$ samples. . . . . . 248

A.3 Additional Monte Carlo information for the $W \rightarrow l \nu$ samples. . . . . 249

A.4 Additional Monte Carlo information for the diboson ( $W W, Z Z, W Z$ )

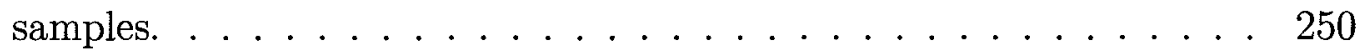

A.5 Additional Monte Carlo information for the single top and all hadronic $t \bar{t}$ samples. . . . . . . . . . . . . . . . . . . 250

A.6 Additional Monte Carlo information for the QCD samples. . . . . . 251

B.1 Example of different ways to calculate the error on an efficiency calculation. . . . . . . . . . . . . . . . . 257 


\section{List of Figures}

2.1 Layout of the LHC accelerator complex. . . . . . . . . . . 17

2.2 ATLAS coordinate system. . . . . . . . . . . . . . 18

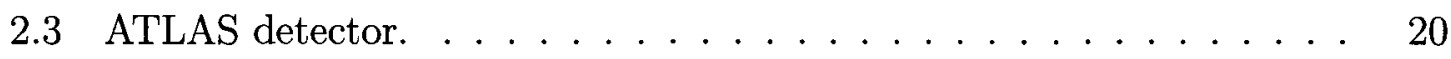

2.4 ATLAS magnet system. . . . . . . . . . . . . . . . 21

2.5 The inner detector: the pixel detector, the silicon central tracker, and the transition radiation tracker. . . . . . . . . . . . . 22

2.6 Layout of the calorimeter systems in ATLAS. . . . . . . . . . 24

2.7 The liquid argon electromagnetic calorimeter. . . . . . . . . . 25

2.8 Amount of material upstream of the presampler and electromagnetic barrel calorimeter. . . . . . . . . . . . . . . 26

2.9 A slice of the tile calorimeter. . . . . . . . . . . . . . 27

2.10 The liquid argon endcap calorimeter. . . . . . . . . . . . . 29

2.11 Components of the muon system. . . . . . . . . . . . . . . 29

3.1 Typical electron energy loss methods for a range of incident electron energies in lead. . . . . . . . . . . . . . . . 36

3.2 Typical photon energy loss methods as a function of incident photon

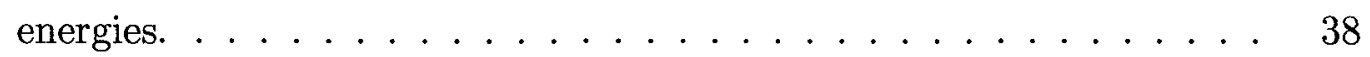

3.3 A diagram of simple electromagnetic shower development, showing pair production from photons and production of Bremsstrahlung photons from electrons, as a function of particle depth. . . . . . . . .

3.4 A schematic of an electrode and the hexagonal electrode pattern in FCal1. . . . . . . . . . . . . . . . . . . . . . . 50

3.5 The front face of FCal1, showing the electrodes, inner and outer edges of the FCal. . . . . . . . . . . . . . . . . . . . 51

3.6 A schematic of the cold electronics for a typical FCall readout channel. 53 
3.7 The location of the five beam impact points on the front face of the FCal. . . . . . . . . . . . . . . . . 56

3.8 Regions of each FCal module that were read out during the test beam. 57

3.9 Test beam experimental beam line setup . . . . . . . . . . . 59

3.10 An example of the response of three upstream scintillators to events that were accepted and rejected. . . . . . . . . . . . . 60

3.11 An example of a subset of the test beam data showing the time-todigital converter units versus timing phase, used to calculate the wrap around constant. . . . . . . . . . . . . . . . .

3.12 Wrap around constant stability over the full test beam data taking period. . . . . . . . . . . . . . . . . . 64

3.13 Normalized pulse shapes for FCal1, FCal2, and FCal3. . . . . . . 66

3.14 Example of the seven samples of the pulse shape taken from a channel in FCal1 with a physics signal from the test beam. Superimposed are fits to a parabola and the optimal filtering technique. . . . . . . 67

3.15 Example distribution of the first sample for one channel in one run. . 70

3.16 Average pedestal values for all data and channels used in this analysis. 71

3.17 Average noise calculated using the first sample of physics and random triggered events, and reconstructed random triggered events, as a function of test beam run number. . . . . . . . . . . . . . .

3.18 Average noise versus channel number averaged over all good runs, where the noise was calculated using the variation in the first sample in physics runs. . . . . . . . . . . . . . . . 74

3.19 Example of energy weighted cluster centers in $x$ and $y . \ldots \ldots .78$

3.20 Difference between projected tracks on the FCall front face and the channel energy weighted cluster centers. . . . . . . . . .

3.21 Forward calorimeter and beam projection chamber alignment versus run number for the $x$ and $y$ coordinates for position $2 . \ldots \ldots \ldots$

3.22 An example of a two-dimensional cluster formed using the topological clustering algorithm. . . . . . . . . . . . . . 83

3.23 $193.1 \mathrm{GeV}$ electron data in position $4 \mathrm{~L}$ before and after event selection. 86

3.24 Electron energy distributions, superimposed with double Gaussian fits,

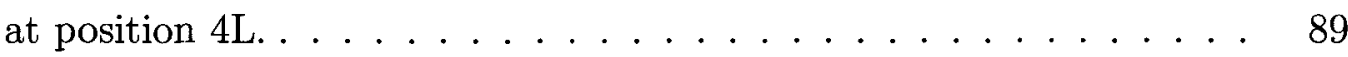

3.25 Electron linearity and residual. . . . . . . . . . . . 90 
3.26 Noise that is subtracted for each beam energy and the noise-subtracted energy resolution for electron data. . . . . . . . . . . .

3.27 Hadron energy distributions, superimposed with double Gaussian fits, at position 4 L. . . . . . . . . . . . . . . .

3.28 Flat weights for each FCal module extracted at each of the beam en-

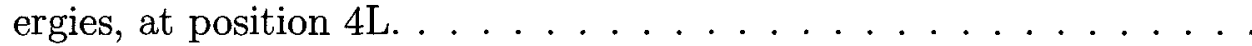

3.29 Hadron linearity with the flat weight hadronic scheme applied, at position 4 L. . . . . . . . . . . . . . . . . . . . 101

3.30 Energy resolution and noise subtracted for hadrons with flat weights applied, position 4L. . . . . . . . . . . . . . . . . . . 102

3.31 Noise-subtracted energy resolution and the residual from best fit linear line, for cylinder clustering and different configurations of the topological clustering for electrons in position $4 \mathrm{~L} . \ldots \ldots$. . . . . . . . 106

3.32 Noise-subtracted energy resolution for cylinder clustering and different configurations of topological clustering for hadrons in position 4L. . . 108

3.33 Linearity for the default cylinder clustering and different configurations of the topological clustering for hadrons in position $4 \mathrm{~L}$. . . . . . . .

3.34 Qualitative picture of the shower axis and cluster center for an arbitrary cluster. . . . . . . . . . . . . . . . . .

3.35 Topological clustering shower shape variables for the highest energy electron and hadron test beam data, position $4 \mathrm{~L} . \ldots . . . . .$.

3.36 A comparison of event cluster multiplicities when the topological cluster splitter is enabled and disabled using the highest energy electron and hadron data in position $4 \mathrm{~L} . \ldots \ldots \ldots \ldots \ldots$

3.37 A comparison of cluster centers in $x$ and $y$ when the topological cluster splitter is enabled and disabled for the highest energy electron and hadron data in position $4 \mathrm{~L} . \ldots \ldots \ldots$. . . . . . . . 118

3.38 A comparison the fraction of energy in the maximum energy cluster when the topological cluster splitter is enabled and disabled using the highest energy electron and hadron data in position 4L. . . . . . . 119

3.39 Expected electronic noise for the ATLAS calorimeters. . . . . . . . . 120

3.40 Selected quantities used to demonstrate the effect of changing the topological cluster splitter parameter called the energy cut, as applied to the highest energy electron and hadron data in position $4 \mathrm{~L}$. . . . . 
3.41 Energy distributions for hadron data and Monte Carlo at position 4L. 130

3.42 Hadron energy response at the electromagnetic scale for the data and Monte Carlo for beam energies from $10 \mathrm{GeV}$ to $200 \mathrm{GeV}$, in position $4 \mathrm{~L} .131$

3.43 Fractional energy deposition in each FCal module for $200 \mathrm{GeV}$ and $60 \mathrm{GeV}$ hadron data and Monte Carlo. . . . . . . . . . . . . . . 132

3.44 The cumulative sum of the energy as a function of radial distance from the impact position for $200 \mathrm{GeV}$ hadron data and Monte Carlo. . . . 133

3.45 The number of channels and channel energies for $200 \mathrm{GeV}$ hadron data and Monte Carlo in FCal1, FCal2, and FCal3. . . . . . . . . 135

3.46 Topological clustering density shower shape variables for hadron test beam data and Monte Carlo. . . . . . . . . . . . . . . . 136

3.47 Topological clustering transverse shower shape variables for hadron test beam data and Monte Carlo. . . . . . . . . . . . . . . . 138

3.48 Topological clustering longitudinal shower shape variables for hadron test beam data and Monte Carlo. . . . . . . . . . . . . . . . . . 139

3.49 Channel positions and energies for channels clustered in one $200 \mathrm{GeV}$ hadron at impact position 1. . . . . . . . . . . . . . . . 142

3.50 Energy distributions at the electromagnetic scale for $200 \mathrm{GeV}$ hadrons at the five impact positions. . . . . . . . . . . . . . . . 143

3.51 The mean reconstructed energy as a function of radial distance from the center of the beam line using track projections. . . . . . . . 146

3.52 The mean reconstructed energy as a function of radial distance from the center of the beam line as calculated with calorimeter only quantities. 147

3.53 Parameterizing the energy lost at the inner edge of the calorimeter with an error function and three free parameters. . . . . . . . . . 149

3.54 Energy distribution for $200 \mathrm{GeV}$ hadrons for position 1, 2, and 3 before and after the inner edge correction. . . . . . . . . . 150

3.55 Mean reconstructed energy as a function of radial distance from the center of the beam line for the data and Monte Carlo. . . . . . . . . 151

3.56 Qualitative description of hadron shower development at the inner edge.151

3.57 The average reconstructed energy versus radial distance from the beam line center for Monte Carlo with real and square beam profiles. . . . 152 
3.58 The fit results for the threshold and width of the inner edge correction function as a function of hadron beam energy, where the impact position on the front face of the FCal was calculated using truth information. 153

3.59 The fit results for the threshold and width of the inner edge correction function as a function of hadron beam energy, where the radial distance from the center of the beam line was calculated using the cluster center.154

3.60 Cluster multiplicity for the data and Monte Carlo at increasing values of $|\eta|$.

3.61 Cluster multiplicity for the data and Monte Carlo at increasing values of $|\eta|$ with the topological cluster splitter enabled. . . . . . . . . . .

4.1 Feynman diagram showing the one-loop correction to the $W$ boson mass arising from fermions in the Standard Model. . . . . . . . . . 163

4.2 Feynman diagrams showing the one-loop correction to the $W$ boson mass arising from the Higgs boson. . . . . . . . . . . . . . . 163

4.3 Leading order Feynman diagrams for the production of top quark pairs at the LHC. . . . . . . . . . . . . . . . . . . . . . . . . . . . 164

4.4 Next to leading order Feynman diagrams for the production of top quark pairs at the LHC. . . . . . . . . . . . . . . . 165

4.5 Feynman diagram for the decay of the top quark. . . . . . . . . . 167

4.6 Top quark $p_{T}$ and $\eta$ distributions for top quarks in the dilepton channel for four Monte Carlo generator samples. . . . . . . . . . . . . . 174

4.7 The $p_{T}$ and $\eta$ for charged leptons $(e, \mu, \tau)$ from $W$ boson decays in $t \bar{t}$ dilepton events for four Monte Carlo generator samples. . . . . . . 175

4.8 True missing transverse energy from the two neutrinos and the invariant mass of the charged leptons in the dilepton channel, from $W$ boson decays in $t \bar{t}$ dilepton events for four Monte Carlo generator samples. .

177

4.9 True jet transverse momentum, $\eta$, and number of jets, using the AntiKt4 jet algorithm, for $t \bar{t}$ dilepton events for four Monte Carlo generator samples . . . . . . . . . . . . . . . . . . . 178

4.10 Geometrical distance and momentum difference between reconstructed electrons and the closest true electrons. . . . . . . . . . . . . 187

4.11 Geometrical distance and momentum difference between reconstructed muons and the closest true muons. . . . . . . . . . . . . . . . 189 
4.12 Calorimeter and track isolation distributions for isolated and nonisolated muons in $t \bar{t}$ dilepton events. . . . . . . . . . . . . . .

4.13 Geometrical distance and momentum difference between reconstructed jets and the closest true jets. . . . . . . . . . . . .

4.14 Reconstructed missing transverse energy difference with respect to the truth and linearity. . . . . . . . . . . . . . . . . . . . . 199

4.15 Event efficiency as a function of transverse missing energy cut. . . . . 200

4.16 Layout of the resistive plate chambers and thin gap chambers used for triggering muons. . . . . . . . . . . . . . . . 206

4.17 $\Delta R$ between reconstructed electrons and the closest trigger region for each trigger level in the e5 medium trigger chain, and $\Delta R$ between reconstructed muons and the closest trigger region for each trigger level in the mu6 trigger chain. . . . . . . . . . . . . . . . . . 210

4.18 Trigger efficiency turn-on curves for the EF_e10_medium and EF_mu10 triggers, for different true $t \bar{t}$ dilepton final states. . . . . . . . . . 212

4.19 Trigger efficiency for electrons with the EF_e10 medium trigger as a function of $E_{\mathrm{T}}$ cut applied to the reconstructed electron, and the muon trigger efficiency using EF_mu10 trigger as a function of $p_{\mathrm{T}}$ cut. . . .

4.20 Electron trigger efficiency as a function of reconstructed electron $E_{\mathrm{T}}$, $\eta, \phi$, and the electron isolation. . . . . . . . . . . . 215

4.21 Muon trigger efficiency as a function of reconstructed muon $p_{\mathrm{T}}, \eta, \phi$, and the calorimeter isolation. . . . . . . . . . . . . . 216

4.22 EF_2e5_medium trigger efficiency as a function of reconstructed elec-

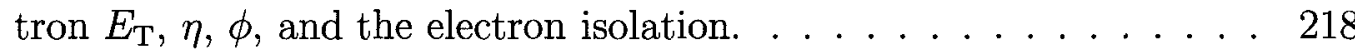

4.23 EF_2mu4 trigger efficiency as a function of reconstructed muon $p_{\mathrm{T}}, \eta$, $\phi$, and muon calorimeter isolation. . . . . . . . . . . . . . 219

4.24 Electron $p_{\mathrm{T}}$, missing $E_{\mathrm{T}}$, the invariant mass of the di-electrons, and the number of jets for the $t \bar{t}$ signal channel and backgrounds for the $e e$ channel.

4.25 Muon $p_{\mathrm{T}}$, and event missing $E_{\mathrm{T}}$, the invariant mass of the di-muons, and the number of jets for the $t \bar{t}$ dilepton signal and backgrounds for the $\mu \mu$ channel. . . . . . . . . . . . . . . . . . . . . 224

4.26 Missing $E_{\mathrm{T}}$ and the number of jets for the $t \bar{t}$ dilepton signal and backgrounds for the $e \mu$ channel. 
4.27 Two-dimensional trigger efficiency for EF_e10_medium and EF_mu10 in $\eta-\phi$ space. . . . . . . . . . . . . . . . . . 234

4.28 An example of the instantaneous luminosity as a function of time using three ATLAS luminosity detectors, with normalization to Pythia MC predictions, during a run on April 4, 2010 . . . . . . . . . . . 239

B.1 Example trigger efficiency distribution when the error on the efficiency is calculated using Poisson, Binomial, and Bayesian statistics. . . . . 258 


\section{List of Acronyms}

$\begin{array}{ll}\text { ADC } & \text { Analogue-to-Digital Converter } \\ \text { ALICE } & \text { A Large Ion Collider Experiment } \\ \text { AOD } & \text { Analysis Object Data } \\ \text { ATLAS } & \text { A Large Toroidal LHC ApparatuS } \\ \text { AU } & \text { Arbitrary Unit } \\ \text { BPC } & \text { Beam Projection Chamber } \\ \text { CEDAR } & \text { ČErenkov Differential counter with Achromatic Ring focus } \\ \text { CERN } & \text { European Organization for Nuclear Research } \\ \text { CMS } & \text { Compact Muon Solenoid } \\ \text { CP } & \text { Charge conjugation and Parity symmetry } \\ \text { CSC } & \text { Cathode Strip Chamber } \\ \text { EF } & \text { Event Filter } \\ \text { EMEC } & \text { ElectroMagnetic Endcap Calorimeter } \\ \text { FCal } & \text { Forward Calorimeter } \\ \text { HEC } & \text { Hadronic Endcap Calorimeter } \\ \text { HLT } & \text { High Level Trigger } \\ \text { HV } & \text { High Voltage } \\ \text { IP } & \text { Interaction Point } \\ \text { L1 } & \text { Level 1 } \\ \text { L2 } & \text { Level } 2 \\ \text { LCG } & \text { LHC Computing Grid } \\ \text { LHC } & \text { Large Hadron Collider } \\ \text { LO } & \text { Leading Order } \\ \text { LUCID } & \text { Luminosity measurement Using Cerenkov Integrating Detector } \\ \text { MBTS } & \text { Minimum Bias Trigger Scintillator } \\ \text { MC } & \text { Monte Carlo } \\ \text { MDT } & \text { Monitored Drift Tube } \\ \end{array}$




$\begin{array}{ll}\text { NLO } & \text { Next-to-Leading Order } \\ \text { NNLO } & \text { Next-to-Next-to-Leading Order } \\ \text { OF } & \text { Optimal Filtering } \\ \text { PDF } & \text { Parton Distribution Function } \\ \text { PSB } & \text { Proton Synchrotron Booster } \\ \text { QCD } & \text { Quantum Chromodynamics } \\ \text { QED } & \text { Quantum Electrodynamics } \\ \text { QGS } & \text { Quark-Gluon String } \\ \text { RPC } & \text { Resistive Plate Chamber } \\ \text { SCT } & \text { Silicon Central Tracker } \\ \text { SM } & \text { Standard Model (of particle physics) } \\ \text { SPS } & \text { Super Proton Synchrontron } \\ \text { TDC } & \text { Time-to-Digital Converter } \\ \text { TGC } & \text { Thin Gap Chamber } \\ \text { TRT } & \text { Transition Radiation Tracker } \\ \text { TTC } & \text { Trigger Timing Clock } \\ \text { WAC } & \text { Wrap Around Constant }\end{array}$




\section{Chapter 1}

\section{The Standard Model of Particle}

\section{Physics}

Particle physics is the theoretical and experimental branch of science that studies the fundamental particles and the interactions between them. Going back to 1869, the publication of the periodic table of elements by D. Mendeleev is one of the earliest attempts to organize 'fundamental' particles (of that time) by their properties. In the original publication there were gaps present in the table that represented predicted, yet undiscovered materials. As the decades passed these holes were filled with the observed materials, until finally 70 years later, after a few false claims, the last naturally occurring element, francium, was discovered and added to the table.

The historical development of the periodic table of elements mirrors that of the current theory of fundamental matter, known as the Standard Model of particle

physics (SM). It too groups fundamental particles into categories based on their theoretical and proven experimental traits. Today, in 2010, there still remains one gap in this SM table; it is a particle called the Higgs boson. 


\begin{tabular}{|c|c|c|c|}
\hline \hline Particle & Electric Charge $(e)$ & Mass $[\mathrm{MeV}]$ & Spin \\
\hline$e$ & -1 & 0.511 & $1 / 2$ \\
$\mu$ & -1 & 105.7 & $1 / 2$ \\
$\tau$ & -1 & 1776.8 & $1 / 2$ \\
\hline$\nu_{e}$ & 0 & $<2 \times 10^{-6}$ & $1 / 2$ \\
$\nu_{\mu}$ & 0 & $<2 \times 10^{-6}$ & $1 / 2$ \\
$\nu_{\tau}$ & 0 & $<2 \times 10^{-6}$ & $1 / 2$ \\
\hline \hline
\end{tabular}

Table 1.1: The electric charge, mass and spin of the leptons. Masses are based on the most current results from the Particle Data Group [1]. The particles are denoted using their variable name. The electric charge is in units of the magnitude of the electron charge $e=1.602 \times 10^{-19} \mathrm{C}$.

\subsection{Quarks and Leptons}

The SM is an effective field theory that has successfully predicted and explained all of the observed fundamental particles and their associated interactions in nature, except for gravity (and the recently confirmed neutrino oscillations). There exist 12 fundamental particles that can be divided into two groups: fermions which form the building blocks of matter and bosons which mediate the interactions between particles. The fermion group can itself be organized into two types, leptons $(l)$ and quarks $(q)$.

There are six known leptons, three are charged and each have a neutral neutrino partner. The lightest, and therefore stable, charged lepton is the electron $(e)$, increasing in mass is the muon $(\mu)$, followed by the tau lepton $(\tau)$. Their corresponding neutrino partners are $\nu_{e}, \nu_{\mu}$, and $\nu_{\tau}$. The neutrinos are known to have a tiny mass, so close to zero that experiments have been unable to measure their mass.. The properties of the six leptons are listed in Table 1.1.1

The second group of fermions, called quarks, are a family with three generations.

\footnotetext{
${ }^{1}$ Throughout this thesis natural units are used, such that $c=\hbar=1$.
} 


\begin{tabular}{|c|c|c|c||c|c|c|c|}
\hline \hline & $\begin{array}{c}\text { Electric } \\
\text { Charge }(e)\end{array}$ & $\begin{array}{c}\text { 'Mass' } \\
{[\mathrm{MeV}]}\end{array}$ & Spin & & $\begin{array}{c}\text { Electric } \\
\text { Charge }(e)\end{array}$ & $\begin{array}{c}\text { 'Mass' } \\
{[\mathrm{MeV}]}\end{array}$ & Spin \\
\hline$u$ & $+2 / 3$ & $1.5-3.3$ & $1 / 2$ & $d$ & $-1 / 3$ & $3.5-6.0$ & $1 / 2$ \\
$c$ & $+2 / 3$ & 1270 & $1 / 2$ & $s$ & $-1 / 3$ & 92.4 & $1 / 2$ \\
$t$ & $+2 / 3$ & 171300 & $1 / 2$ & $b$ & $-1 / 3$ & 4200 & $1 / 2$ \\
\hline \hline
\end{tabular}

Table 1.2: The electric charge, mass and spin of quarks [1]. The particles are denoted using their variable name. The electric charge is in units of the magnitude of the electron charge $e=1.602 \times 10^{-19} \mathrm{C}$. They are grouped together in their doublet pairs.

There is the charge $+2 / 3$ family consisting of the up $(u)$, charm $(c)$, and top $(t)$ quarks that are partnered with the $-1 / 3$ charged quarks the down $(d)$, strange $(s)$, and bottom (b) quarks.

Quarks can be combined in a triplet state $(q q q, \bar{q} \bar{q})$ to form baryons, or quark and antiquark pairs $(q \bar{q})$ to form mesons. The fractional charge of the quarks ensures that hadrons (baryons and mesons) have integer electric charge (in units of the electron charge). For example, the proton is composed of the uud quarks; by simply summing their charges the proton obtains a charge of +1 . The masses, charges, and spins of the quarks are listed in Table 1.2. Quarks do not exist in a free state, therefore the masses quoted are calculated indirectly from hadronic properties (many measurable quantities such as cross-sections have some dependence on the mass of the interacting particles).

Each of the twelve fundamental fermions have an antimatter partner with the same mass and spin, however the other quantum numbers (such as charge) differ by a factor of -1 . 


\subsection{Fundamental Interactions}

In the SM particle interactions proceed via the exchange of force carrier particles, the type of which is determined by the interaction. The four known interactions that exist between the particles are electromagnetic, strong, weak, and gravitational. Although, as mentioned, only the first three are included in the SM.

\subsubsection{Electromagnetism}

The electromagnetic interaction is quantized in a theory called quantum electrodynamics (QED). QED demands local gauge invariance of spin 1/2 particles, with charge $Q e$ (where $Q$ is the electron charge in Coulombs):

$$
\psi \rightarrow \psi e^{\imath Q \lambda(x)}
$$

The transformation $e^{\imath Q \lambda(x)}$ depends on the (local) location in space-time $x$. Gauge invariance implies the physically observed quantities, such as charge and cross-sections, remain unchanged after this local phase transformation. This demand is satisfied by the introduction of a vector field $A_{\mu}$ which itself must transform as:

$$
A_{\mu} \rightarrow A_{\mu}+\partial_{\mu} \lambda
$$

to maintain local gauge invariance. The Lagrangian describing $\psi$ and $A_{\mu}$ can be written as:

$$
\mathcal{L}_{\mathrm{QED}}=\bar{\psi}\left(i \gamma^{\mu} D_{\mu}-m\right) \psi-F_{\mu \nu} F^{\mu \nu}
$$

where $\bar{\psi}=\psi^{\dagger} \gamma^{\circ}$, and $\gamma^{\mu}$ are the Dirac matrices. Also in $\mathcal{L}_{\mathrm{QED}}$ are the covariant derivative defined as $D_{\mu}=\partial_{\mu}+i Q A_{\mu}$, and the term $F_{\mu \nu}=\partial_{\mu} A_{\nu}-\partial_{\nu} A_{\mu}$. This 
Lagrangian describes the interaction between massive, charged spin 1/2 particles (with mass $m$ ) and a massless vector field (the photon), with coupling proportional to $Q$. The $F_{\mu \nu} F^{\mu \nu}$ term in the Lagrangian can be used to produce Maxwell's equations. No mass term can be added for the vector field in the Lagrangian without violating local gauge invariance.

\subsubsection{Strong Interaction}

Quantum chromodynamics (QCD) is the theory of strong interactions, which are only experienced by quarks and gluons. It is the force responsible for holding together quarks in baryons and mesons. To quantify this force, quarks have an additional quantum number, a colour charge which is the source of the field. There are three of these colours which are typically classified as red, green, and blue. Quarks are colour triplets and combine to form colourless hadrons.

The theory requires the Lagrangian describing a quark $q$ of some colour and flavour be invariant under a local gauge invariance of an $S U(3)$ transformation:

$$
q \rightarrow q e^{\left(-i g_{s} \sum_{\imath=1}^{8} T_{\imath} \chi_{\imath}(x)\right)}
$$

with $T_{i}=\lambda_{i} / 2$ where $\lambda_{i}$ are the $3 \times 3$ unitary traceless matrix generators of the $S U(3)$ group, $g_{s}$ is the QCD colour coupling strength, and $\chi_{\imath}(x)$ the arbitrary local transformations. In the following the sum over $i=1, \ldots, 8$ is implied where not explicitly written.

The $S U(3)$ invariant QCD Lagrangian for one flavour of quark is written as:

$$
\mathcal{L}_{\mathrm{QCD}}=-\frac{1}{4} F_{\mu \nu}^{i} F^{i \mu \nu}+i \bar{q} \gamma^{\mu}\left(D_{\mu}\right) q-m \bar{q} q
$$


As before the covariant derivative is necessary for local gauge invariance and here is equal to: $D_{\mu}=\partial_{\mu}+i g_{s} T_{2} A_{\mu}^{2}$; it gives rise to the coupling between the quarks and gluons with coupling strength proportional to $g_{s}$. The $F_{\mu \nu}^{2}$ describe the selfinteractions of the eight $A_{\mu}^{2}$ (gluon force carrier) fields:

$$
F_{\mu \nu}^{2}=\partial_{\mu} A_{\nu}^{2}-\partial_{\nu} A_{\mu}^{2}-g_{s} f_{\imath \jmath k} A_{\mu}^{\jmath} A_{\nu}^{k}
$$

containing $f_{\imath \jmath k}$, the structure constants of the group. There are triplet and quartic gluon couplings, unlike QED; a consequence of the fact that the gluons themselves carry colour charge (unlike the photon which is electrically neutral).

\subsection{3 (Electro)Weak Interaction and the Higgs Mechanism}

The charged weak interaction couples to the left-handed components of quarks and leptons. A left-handed particle is one in which the direction of its spin is opposite to its momentum, where as the spin and momentum are aligned for right-handed particles. The leptons in Table 1.1 can be written as a left-handed doublet:

$$
\left(\begin{array}{c}
e \\
\nu_{e}
\end{array}\right)_{L}\left(\begin{array}{c}
\mu \\
\nu_{\mu}
\end{array}\right)_{L}\left(\begin{array}{l}
\tau \\
\nu_{\tau}
\end{array}\right)_{L},
$$

and a right-handed singlet, to which only the charged massive leptons contribute (assuming the neutrino is massless): $(e)_{R} \quad(\mu)_{R} \quad(\tau)_{R}$. Similarly, the quarks in Table 1.2 can be written as right-handed singlets and left-handed doublets. For example, the right-handed quark singlets for the first family are $(u)_{R}$ and $(d)_{R}$. The 
left-handed quark doublets are written as:

$$
\left(\begin{array}{l}
u \\
d^{\prime}
\end{array}\right)\left(\begin{array}{l}
c \\
s^{\prime}
\end{array}\right)\left(\begin{array}{c}
t \\
b^{\prime}
\end{array}\right),
$$

with $d_{\imath}^{\prime}=\sum_{\jmath} U_{\imath, \jmath} d_{\jmath}$, where the $d_{\jmath}$ are the left-handed down-type quarks $(d, s, b)$ and $U_{\imath, 3}$ is a $3 \times 3$ matrix representing the degree of mixing between the left-handed down-type families.

The symmetry group that allows the interaction between weak bosons and the left-handed doublets is the $S U(2)$ group. Following from the examples of QED and $\mathrm{QCD}$, requiring local gauge invariance of the doublet wavefunction would yield a Lagrangian that would not contain mass terms for the gauge bosons responsible for mediating the field. However the gauge mediators of the weak force are the massive $Z^{o}$ and $W^{ \pm}$bosons. If a mass term were to be added for the bosons, then the theory would become unrenormalizable, meaning that calculations of observed quantities such as cross-sections would contain infinite terms that could not be absorbed into a re-definition of the couplings. This is clearly a problem because the observed gauge bosons are quite massive.

To account for this the electroweak theory incorporating the Higgs mechanism was developed. The electroweak theory was developed by S. Glashow, S. Weinberg, and A. Salam in the 1960s and combines electromagnetism and the weak interaction under the $S U(2)_{L} \times U(1)_{Y}$ symmetry; the subscript $L$ on $S U(2)$ signifies it is the left-handed doublets that transform with this symmetry, and the subscript $Y$ denotes hypercharge, another quantum number carried by the fermions [2-4]. The electroweak Lagrangian can be broken down into pieces:

$$
\mathcal{L}_{\text {EW }}=\mathcal{L}_{\text {gauge }}+\mathcal{L}_{\text {Higgs }}+\mathcal{L}_{\text {fermion }}+\mathcal{L}_{\text {Yukawa }}
$$


Each Lagrangian will be described in the following paragraphs.

The gauge Lagrangian $\mathcal{L}_{\text {gauge }}$ describes the kinetic energy terms of the vector fields (of which there are four):

$$
\mathcal{L}_{\text {gauge }}=-\frac{1}{4} G_{\mu \nu} G^{\mu \nu}-\frac{1}{4} F_{\mu \nu}^{\imath} F^{\imath, \mu \nu}
$$

with $G_{\mu \nu}=\partial_{\mu} B_{\nu}-\partial_{\nu} B_{\mu}$ the kinetic energy term for the $U(1)_{Y}$ vector field $B_{\mu}$, and $F_{\mu \nu}^{\imath}=\partial_{\mu} W_{\nu}^{\imath}-\partial_{\mu} W_{\mu}^{\imath}+g \epsilon^{\imath \jmath} k W_{\mu}^{\jmath} W_{\nu}^{k}$ for $S U(2)_{L}$ with the three vector fields $W_{\mu}^{\imath}$ $(i=1,2,3)$. Here $g$ is the coupling between the $W_{\mu}^{\imath}$ fields, and $\epsilon^{\imath \jmath k}$ are the structure constants for the $S U(2)$ group.

To give mass to three of the gauge bosons in such a way that the Lagrangian remains gauge invariant, the gauge bosons are coupled to a complex scalar doublet $S U(2)_{L}$ field $\Phi$ with a Lagrangian:

$$
\mathcal{L}_{\text {Higgs }}=\left(D_{\mu} \Phi\right)^{\dagger}\left(D_{\mu} \Phi\right)-V\left(\Phi^{\dagger} \Phi\right)
$$

where the first term is the kinetic term of the scalar field and the second term the potential energy of the scalar field. In the kinetic term, the gauge invariant covariant derivative is:

$$
D_{\mu} \Phi=\left(\partial_{\mu}-i g \frac{\vec{\sigma}}{2} \cdot \vec{W}_{\mu}-i \frac{g^{\prime}}{2} B_{\mu}\right) \Phi
$$

where $\vec{\sigma}=\left(\sigma_{1}, \sigma_{2}, \sigma_{3}\right)$ are the three Pauli matrices. The coupling $g$ appears again, and is called the isospin coupling. The other coupling constant $g^{\prime}$ is introduced and is the weak hypercharge coupling between the $U(1)_{Y}$ field $\left(B_{\mu}\right)$ and the scalar field $(\Phi)$.

The simplest gauge invariant renormalizable potential for the scalar field is written 
as:

$$
V\left(\Phi^{\dagger} \Phi\right)=\mu^{2}\left|\Phi^{\dagger} \Phi\right|+\lambda\left(\left|\Phi^{\dagger} \Phi\right|^{2}\right)
$$

where $\mu$ and $\lambda$ are constants. The non-trivial solution to the potential that satisfies the goal of the theory (massive gauge bosons) finds a minimum value of the potential to occur at $\left|\Phi_{\mathrm{o}}\right|=v / \sqrt{2}$, where $v^{2}=-\mu^{2} / \lambda, \mu^{2}<0$ and $\lambda>0$. Choosing a gauge in which only the real neutral component of $\Phi$ is nonzero yields:

$$
\Phi=\left(\begin{array}{c}
0 \\
\frac{1}{\sqrt{2}}(v+H(x))
\end{array}\right)
$$

where $v$ is a constant known as the vacuum expectation value representing the minimum value of the potential, and the field $H(x)$ is the perturbation away from the vacuum state is called the Higgs boson.

Doing a change of basis from $W_{\mu}^{\imath}$ and $B_{\mu}$ into the physically observed fields $W^{ \pm}$, $Z$ and $A_{\mu}$ via the transformations:

$$
\begin{aligned}
W_{\mu}^{ \pm} & =\frac{1}{\sqrt{2}}\left(W_{\mu}^{1} \mp i W_{\mu}^{2}\right) \\
Z_{\mu} & =\frac{1}{\sqrt{g^{2}+g^{2}}}\left(g W_{\mu}^{3}-g^{\prime} B_{\mu}\right) \\
A_{\mu} & =\frac{1}{\sqrt{g^{2}+g^{\prime 2}}}\left(g^{\prime} W_{\mu}^{3}+g B_{\mu}\right) .
\end{aligned}
$$

Expanding the kinetic and potential energy terms of Equation 1.3, yields two massive charged vector fields, one massive neutral vector field, and one massless vector field:

$$
\begin{aligned}
M_{W^{ \pm}} & =\frac{g v}{2} \\
M_{Z} & =\frac{v}{2} \sqrt{g^{2}+g^{2}} \\
M_{A} & =0
\end{aligned}
$$


The potential term in Equation 1.4 predicts one massive scalar field, the Higgs boson with mass $M_{H}^{2}=-\mu^{2}=2 v^{2} \lambda$. The theory is said to have undergone spontaneous symmetry breaking because the Higgs field has acquired a non-zero vacuum expectation value, breaking the symmetry in the ground state. The photon $A_{\mu}$ remains massless as it does not interact with the Higgs field, therefore the $U(1)$ electromagnetic component remains gauge invariant.

The charged leptons and quarks are also massive particles and have left-handed and right-handed components that couple differently to the massive gauge bosons. To ensure gauge invariance and generate fermion masses, the same Higgs doublet is used in the fermion sector. Under a rotation in $S U(2)$ of the left-handed doublets these fermions undergo the transformation:

$$
\psi_{L} \rightarrow e^{-i \tau_{2} \theta_{2}(x)} \psi_{L}
$$

where $\tau_{i}=\sigma_{i} / 2$ are the three generators of the $S U(2)_{L}$ group. The right-handed singlets remained unchanged. Under the $U(1)_{Y}$ transformation the fermions undergo:

$$
\psi_{L, R} \rightarrow e^{-i Y_{L, R} \theta(x)} \psi_{L, R}
$$

where the left-handed and right-handed pieces transform with the different hypercharge $(Y)$ values. The hypercharge and third-component of the isospin vector are related by:

$$
Y=2\left(Q-T_{3}\right)
$$

where $Q$ is the electric charge of the particle, and $T_{3}$ is the third component of the isospin vector with values of $\pm \frac{1}{2}$ for the weak left-handed doublets and zero for the singlets. 
Returning to Equation 1.2, the kinetic energy term in the Lagrangian for fermions is:

$$
\mathcal{L}_{\text {fermion }}=i\left(\bar{\psi}_{L} D_{\mu} \gamma^{\mu} \psi_{L}+\bar{\psi}_{R} D_{\mu} \gamma^{\mu} \psi_{R}\right)
$$

with $D_{\mu} \psi_{L}=\left(\partial_{\mu}-i g \frac{\vec{\sigma}}{2} \cdot \vec{W}-i g^{\prime} \frac{Y_{L}}{2} B_{\mu}\right) \psi_{L}$ and $D_{\mu} \psi_{R}=\left(\partial_{\mu}-i g^{\prime} \frac{Y_{R}}{2} B_{\mu}\right)$. Expanding the terms reveals the allowed couplings between the fermions of each handed-ness and gauge bosons.

The most general term to generate the fermion mass terms is the Yukawa Lagrangian:

$$
\mathcal{L}_{\text {Yukawa }}=y_{e} \bar{l}_{L} \Phi e_{R}+y_{d} \bar{q}_{L} \Phi d_{R}+y_{u} \bar{q}_{L} \tilde{\Phi} u_{R}+\text { h.c. }
$$

where $l_{L}, q_{L}$ are the $S U(2)$ left-handed lepton and quark doublets, and $e_{R}, u_{R}, d_{R}$ are the right-handed singlets previously defined. The conjugate Higgs doublet is $\tilde{\Phi}=i \sigma_{2} \Phi^{*}$. The first term in the equation is for the leptons, the second term is for the down-type quarks, and the third term is for the up-type quarks (shown here for the first family of each). There is only one term for the charged leptons as neutrinos are assumed to be massless and therefore do not have right-handed states. The variables $y_{e, d, u}$ are the Yukawa couplings of the fermions to the Higgs field, and are related to the mass of a fermion by $y_{f}=\frac{M_{f}}{\sqrt{2} v}$. The coupling of the Higgs boson $H$ to a fermion (found by expanding the scalar field using Equation 1.5) is proportional to the mass of the fermion.

\section{$1.3 \quad$ Summary}

The SM combines the gauge groups $S U(2)_{L} \times U(1)_{Y} \times S U(3)_{C}$ to predict the gauge bosons (Table 1.3) that mediate the interactions between particles. The theory also gives rise to the masses of bosons and fermions via coupling to the Higgs scalar field. 


\begin{tabular}{|l|c|c|c|c|c|}
\hline \hline Force & Mediator & Electric Charge & Mass [MeV] & Spin & Relative Strength \\
\hline electromagnetic & $\gamma$ & 0 & 0 & 1 & $10^{-2}$ \\
weak & $Z, W^{ \pm}$ & $0, \pm 1$ & $91.1876,80.398$ & 1 & $10^{-5}$ \\
strong & eight gluons & 0 & 0 & 1 & 1 \\
\hline \hline
\end{tabular}

Table 1.3: Properties of the gauge bosons that mediate the forces between the quarks and leptons [1]. The relative strengths for the forces are at distance of $10^{-18} \mathrm{~cm}$ [5], not included in this table is gravity which is believed to be mediated by the graviton (currently unobserved) and has a relative strength of $10^{-39}$.

The masses of the gauge bosons are related by parameters of their coupling strengths.

The theory has been quite successful, and coupling constants and masses have been measured with fine precision, yet it does not explain all of the observed phenomena. It does not explain the mass heirarchy of the fermions, massive neutrinos, or why there are only three families. It does not account for dark matter or dark energy in the Universe. And it does not explain gravity. Alternative theories exist that require more free parameters and fine tuning of these parameters to get back the observed physics behavior that has been explained by the SM. Finally, the cornerstone of the theory relies on the existence of the Higgs boson, the only particle of the theory that has not yet been observed.

\subsection{A Look Ahead}

This chapter has described the SM and has tried to motivate the need to continue the investigation of its framework. The remainder of this thesis examines a large spectra of phenomena associated to experimental particle physics, the field which tests the predictions of the SM. The analysis is presented in the context of the ATLAS experiment, which will be described in more detail in the following chapter, Chapter 2. The ATLAS detector is an experiment part of the Large Hadron Collider, designed 
to search for the Higgs boson, to pursue specific SM measurements, and also to test for theories beyond the SM. All aspects of the detector, from hardware to software, must be understood and optimized to achieve the physics goals at hand. Chapter 3 presents the calibration of one sub-detector in ATLAS, called the forward calorimeter, using beam test data and Monte Carlo studies. The last chapter, Chapter 4, uses Monte Carlo data sets to study the top quark production cross-section using the dilepton final state in ATLAS with $200 \mathrm{pb}^{-1}$ (at $\sqrt{s}=7 \mathrm{TeV}$ ). Particular attention is given to different trigger configurations that might be used in ATLAS to detect the signal of interest, and how trigger efficiencies can be obtained and applied to the analysis. Some studies are made about the associated systematic uncertainties to the top quark cross-section measurement. 


\section{Chapter 2}

\section{The Large Hadron Collider and the ATLAS Detector}

The Large Hadron Collider (LHC) is a particle accelerator, located at the European Organization for Nuclear Research (CERN) on the French-Swiss border, near Geneva, Switzerland. Some of the primary goals of the LHC are to search for the Higgs boson, make precision SM measurements, and to seek evidence of alternative theories that may explain such things as gravity and dark matter. These goals are achieved by colliding particles together at extremely high energy density, conditions believed to exist immediately after the Big Bang. Working in conjunction with the accelerator are several detectors placed at locations where these accelerated particles collide. The detectors reconstruct the particles trajectories back to the initial interactions to understand the underlying physics of each collision. 


\subsection{The Large Hadron Collider}

The LHC is designed to accelerate protons and heavy ions to record energies. At a depth of $\sim 100 \mathrm{~m}$ underground, the LHC forms a ring of circumference $27 \mathrm{~km}$, and has two beam pipes for particles beams to travel in opposite directions. There are 1232 dipoles that operate at superconducting temperatures $(1.9 \mathrm{~K})$, providing a magnetic field of $8.36 \mathrm{~T}$, to guide the particles in circular arcs to these energies.

The protons achieve maximum energies of $7 \mathrm{TeV}$ via several stages of acceleration, as depicted in Figure 2.1. In the first stage protons are accelerated to $50 \mathrm{MeV}$ by a linear accelerator (LINAC). They are then injected into the Proton Synchrotron Booster (PSB) to attain particle energies of $1.4 \mathrm{GeV}$. They are then passed to Proton Synchrotron (PS), where they are boosted to energies of $26 \mathrm{GeV}$. The final stage before injection into the LHC is completed by the Super Proton Synchrotron (SPS) and brings the protons to energies of $450 \mathrm{GeV}$. The protons are then fed into the LHC where they can be accelerated to achieve maximum energies of $7 \mathrm{TeV}$.

The final goal of colliding protons with center of mass energies of $\sqrt{s}=14 \mathrm{TeV}$ will be achieved after several years of LHC running, with the beam energy incrementally rising. The initial energy steps took place in the fall 2009 and saw proton-proton $(p p)$ collisions with $\sqrt{s}<3 \mathrm{TeV}(900 \mathrm{GeV}, 1.18 \mathrm{TeV}, 2.36 \mathrm{TeV})$. On March 19, 2010 the LHC collided protons with energies of $\sqrt{s}=7 \mathrm{TeV}$. The LHC is expected to collide protons at $\sqrt{s}=7 \mathrm{TeV}$ until the end of 2011. Following this, there is expected to be a year long shutdown to prepare the accelerator for increased energy. When the LHC begins colliding particles in 2013 it is expected that the energy will be $\sqrt{s}=10 \mathrm{TeV}$, or possibly even the design energy of $14 \mathrm{TeV}$.

The particles in the machine are grouped together in bunches, with a maximum of 2808 bunches in each direction. Each bunch can hold a maximum of $1.1 \times 10^{11}$ protons, 


\begin{tabular}{|l|c|}
\hline \hline Property & Value \\
\hline Ring circumference & $26.658 \mathrm{~km}$ \\
Number of particles per bunch & $1.1 \times 10^{11}$ \\
Number of bunches & 2808 \\
Bunch spacing & $25 \mathrm{~ns}$ \\
Beam energy & $7 \mathrm{TeV}$ \\
Luminosity & $10^{34} \mathrm{~cm}^{-1} \mathrm{~s}^{-1}$ \\
Number of dipoles & 1232 \\
Rotation frequency & $11245 \mathrm{~Hz}$ \\
\hline \hline
\end{tabular}

Table 2.1: Selected properties of the LHC at the designed specifications. The values for the number of particles per bunch, number of bunches, bunch spacing, beam energy, and luminosity are quoted for the design specifications which will be achieved after several years of LHC operation.

and the bunches are separated by $25 \mathrm{~ns}$. At peak luminosity every bunch crossing is expected to result in $\sim 20$ inelastic $p p$ collisions per bunch crossing, yielding the production of many outward going particles from overlapping events creating what is termed pileup. A summary of some of the LHC parameters is given in Table 2.1.

The counter-rotating particle beams are focussed to collide at four points around the ring, corresponding to the location of four detectors, indicted by the filled circles in Figure 2.1. ALICE (A Large Ion Collider Experiment) has been created to study phenomena of quark-gluon plasma in heavy ion collisions. ATLAS (A Large Toroidal LHC Apparatus) and CMS (Compact Muon Solenoid) are two general purpose detectors established to study the SM and beyond the SM physics. The fourth detector, $\mathrm{LHCb}$, is a spectrometer designed to study the violation of the combined charge conjugation and parity (CP) symmetry, and rare particle decays. 


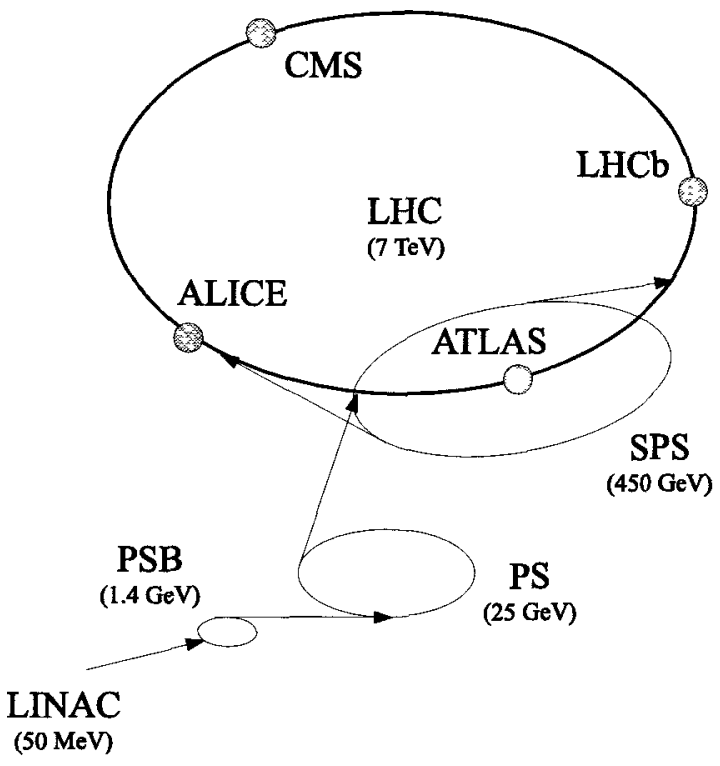

Figure 2.1: Layout of the LHC accelerator complex, including the various accelerators and detectors.

\section{$2.2 \quad$ ATLAS}

The ATLAS collaboration was formed in 1992, and is currently made of more than 3000 people from 38 countries. The ATLAS detector has several physics goals, however one of the most important ones is the discovery or exclusion of the SM Higgs boson. Depending on its mass, the SM Higgs boson preferentially decays into certain final states which are then observed in the detector. Recent experimental and theoretical constraints limit the Higgs boson mass to lie between $114 \mathrm{GeV}$ and $185 \mathrm{GeV}$ (at the $95 \%$ confidence limit), excluding the mass range from $162 \mathrm{GeV}$ and $166 \mathrm{GeV}$ [6]. For a Higgs boson with a mass less than $140 \mathrm{GeV}$ the preferred decay is into $b \bar{b}$. This channel is difficult to resolve in proton-proton collisions that yield many hadrons in the final state. However, the Higgs boson can be produced in association with distinct jets in the forward region, near the beam line. Another interesting and possibly 

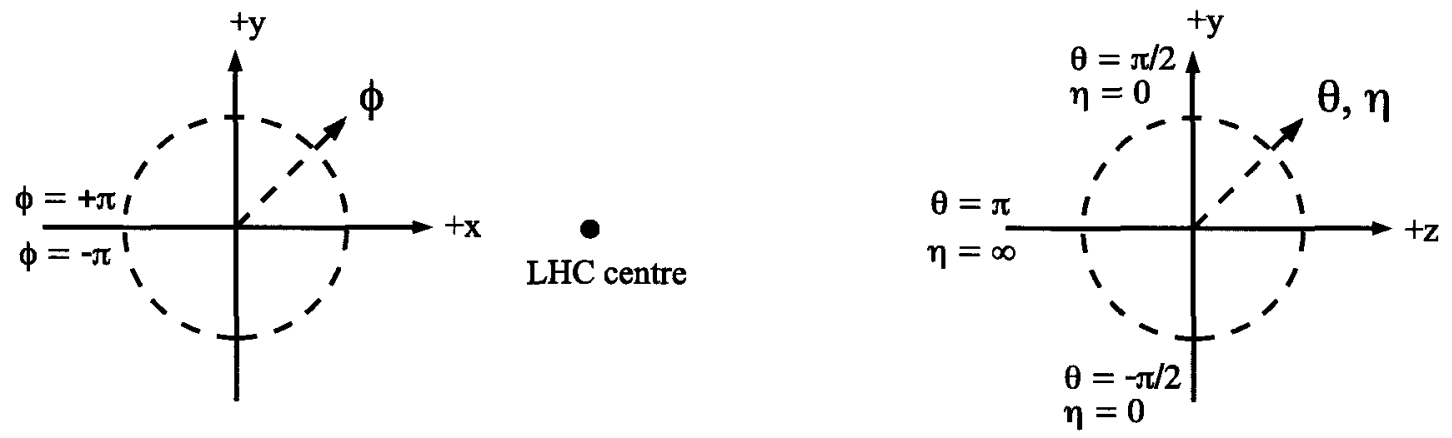

Figure 2.2: The ATLAS coordinate system.

clearer experimental signature for a low mass Higgs boson is its decay into two photons. For a Higgs boson with a mass greater than $140 \mathrm{GeV}$ the largest branching ratios are into final states of $W W$ and $Z Z$. The best observation channel will then be the subsequent decay of $Z Z$ into four leptons.

These preferred Higgs boson decay channels have driven the design of the ATLAS detector. The design is general enough such that the study of other physics analyses will be possible. Some analyses will make precise measurements of SM properties and others will search for physics that supports evidence for theories beyond the SM.

\subsubsection{Coordinate System}

The coordinate system in ATLAS is defined with the $z$-axis along the beam line. The $+x$-axis points toward the centre of the LHC ring, and the $+y$-axis points upwards. The azimuthal angle $\phi$ is measured with respect to the $+x$-axis; it increases to $+\pi$ radians going counterclockwise around the $z$-axis, and $-\pi$ radians in the clockwise direction. The polar angle $\theta$ is measured with respect to the $+z$-axis. In high energy hadron colliders $\theta$ is often written in terms of a unit-less quantity called pseudo- 
rapidity, defined as:

$$
\eta=-\ln \left[\tan \left(\frac{\theta}{2}\right)\right]
$$

It is an approximation of rapidity $(y)$ for massless particles, where

$$
y=\frac{1}{2} \ln \left(\frac{E+p_{L}}{E-p_{L}}\right)
$$

with $E$ the energy and $p_{L}$ the longitudinal momentum of the particle of interest. For hadron colliders it is not possible to measure the longitudinal components of the momentum, but it is possible to measure the angles with respect to the beam axis, therefore $\eta$ is preferred. A value $\eta=0$ corresponds to $\theta=\pi / 2$, and $\eta=\infty$ to $\theta=0$. Values of high $|\eta|$ are regions close to the LHC beam pipe. Figure 2.2 shows the coordinate convention used for the ATLAS detector.

The ATLAS detector, shown in Figure 2.3, is constructed from several subsystems, working together to provide good momentum measurements, track reconstruction, and calorimeter energy resolution. Each of these subsystems will be discussed in more detail below. The massive ATLAS detector is $44 \mathrm{~m}$ long, $25 \mathrm{~m}$ high, and weighs 7000 tonnes. A very detailed discussion of the ATLAS detector can be found in [7]. Many of the ATLAS subsystems are broken up into two sections: a barrel and an endcap. The barrel sections typically form an open-ended cylinder shape around the core. And the endcap regions typically seal off the detector at each end.

\subsubsection{Magnet System}

There are four superconducting magnet systems in ATLAS, shown in Figure 2.4. The central solenoid, which generates a $2 \mathrm{~T}$ magnetic field, lies between the inner detector and the calorimeters, with an inner and outer radius of $1.23 \mathrm{~m}$ and $1.28 \mathrm{~m}$, 


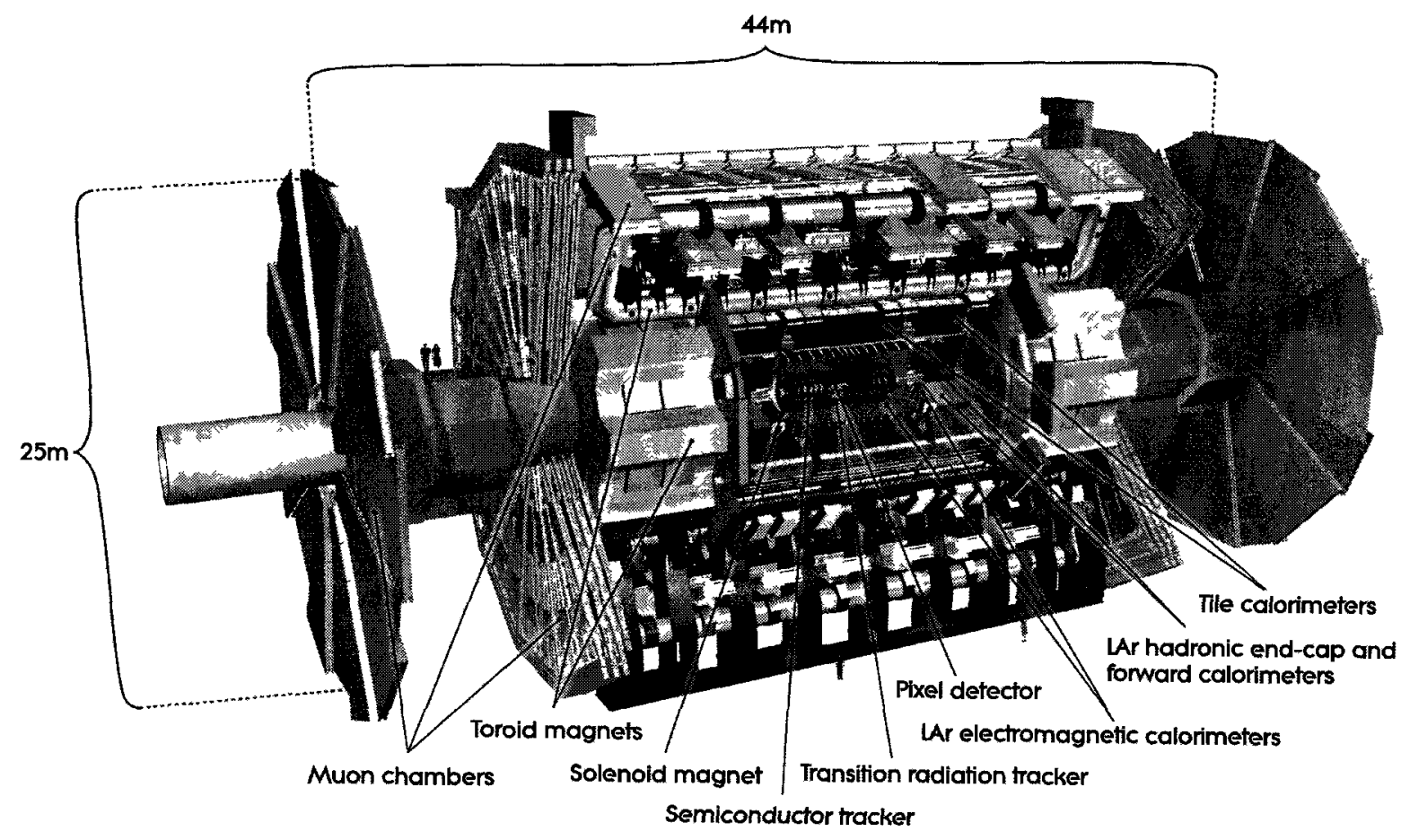

Figure 2.3: Layout of the ATLAS detector.

respectively, from the center of the beam line. The toroid system lies outside the hadronic calorimeters. It is composed of the barrel toroid system plus two endcap toroids, deflect charged particle trajectories to assist in muon identification. The barrel toroid is a system of eight coils, placed in individual cryostats. They are 25 $\mathrm{m}$ long and sit equally spaced, radially around the beam line, with inner and outer radii of $4.7 \mathrm{~m}$ and $10.05 \mathrm{~m}$. They provide a magnetic field of approximately $0.5 \mathrm{~T}$ in the region of $0<|\eta|<1.4$. Each endcap toroid is also composed of eight coils that lie perpendicular the beam line and are enclosed in the same cryostat. The endcap toroid generates a $1.0 \mathrm{~T}$ magnetic field between $1.6<|\eta|<2.7$. 


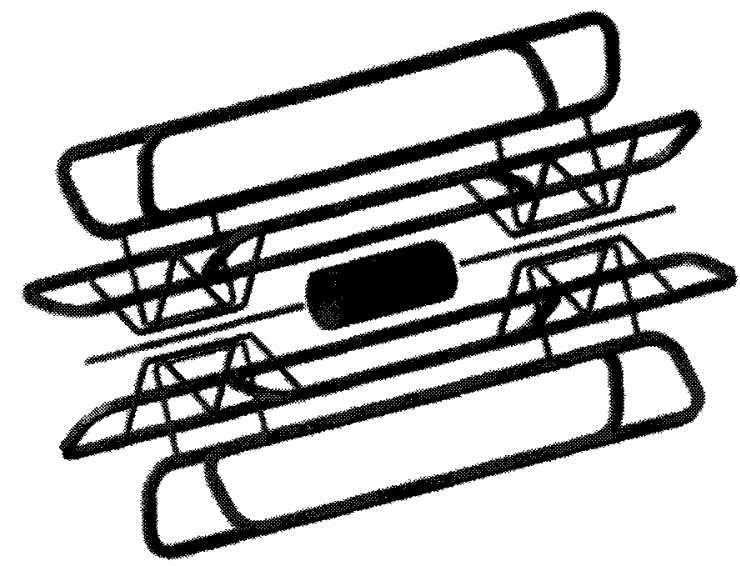

Figure 2.4: The ATLAS magnet system. The central solenoid is in the center, while the toroid system is outside.

\subsubsection{Inner Detector}

The inner detector is the closest subsystem to the collision point. It serves to reconstruct trajectories of charged particles, and measure the positions of primary and any secondary vertices. The identification of secondary vertices is used to reconstruct heavy quarks and $\tau$-leptons that travel a very limited distance from the interaction point (IP) before they decay. The inner detector combined with the magnetic field from the solenoid allow for measurements of the momentum, charge, and direction of charged particles with $|\eta|<2.5$. The inner detector is made of three systems that can be seen in Figure 2.5. Going from the inner-most system outward, is the silicon pixel detector, the silicon central tracker (SCT), and the transition radiation tracker (TRT). Each is broken up into a barrel region, with layers running concentric with the beam line, and endcap disks that are perpendicular to the beam axis.

The pixel detector has the highest granularity which is provided by pixel semiconductor silicon wafers with an area of $50 \times 400 \mu \mathrm{m}^{2}$. It has 80.4 million readout channels that are located on three barrel layers and three endcap disks, spanning radii between $45 \mathrm{~mm}$ and $240 \mathrm{~mm}$ from the center of the beam line, with $|\eta|<2.5$. The 


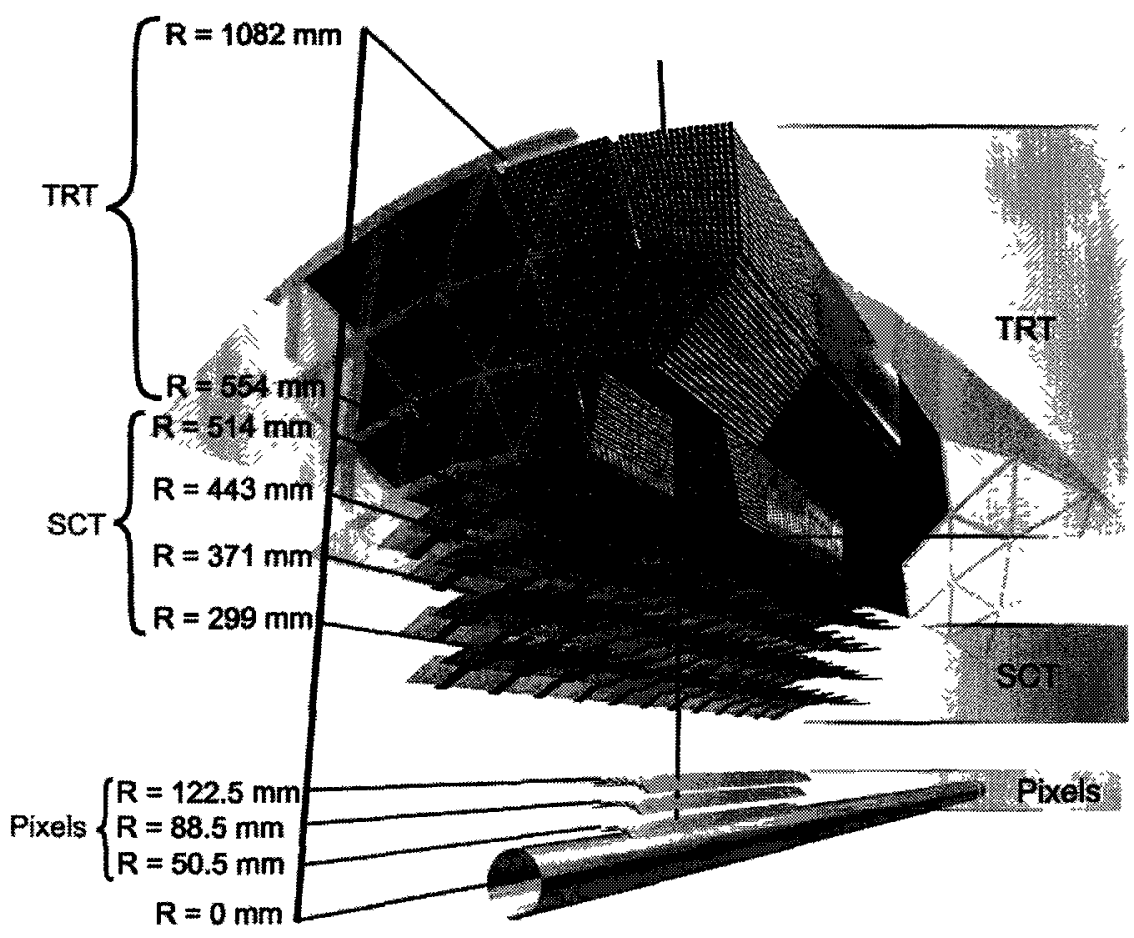

Figure 2.5: A schematic of the elements in the barrel inner detector: the pixel detector, the silicon central tracker, and the transition radiation tracker. For each subsystem the radial distance from the centre of the beam line is indicated.

position resolution of the pixel detector is $10 \mu \mathrm{m}$ in $R-\phi$ and $115 \mu \mathrm{m}$ in $z$.

Surrounding the pixel detector is the SCT, which is made of semiconductor silicon strips. The strips are positioned on four barrel layers and nine endcap disks, yielding a total of 6.3 million readout channels within $|\eta|<2.5$. In the barrel the strips lie parallel to the beam line and in the endcap they lie radially. The resolution of the SCT strips is $17 \mu \mathrm{m}$ in the $R-\phi$ coordinate and $580 \mu \mathrm{m}$ in $z$.

The TRT is composed of $144 \mathrm{~cm}$ long straw tubes in the barrel and $37 \mathrm{~cm}$ long straw tubes in the endcap, used to measure the $R-\phi$ coordinates of charged particles out to $|\eta|<2.0$. The TRT measures the transition radiation produced by relativistic charged particles as they cross regions with different dielectric constants (layers interleaved between the tubes). In the TRT the transition radiation is detected by a xenon gas mixture in the tubes. The probability for a charged particle to emit tran- 
sition radiation is proportional to its Lorentz factor $\gamma=E / m$. For particles of the same energy, lighter particles like electrons will generate more radiation than heavier particles like pions. Hence the TRT is a good detector for separating electrons from hadrons. The TRT provides continuous track measurements between $R=554 \mathrm{~mm}$ and $R=1082 \mathrm{~mm}$ in the barrel (and $R=617 \mathrm{~mm}$ and $R=1106 \mathrm{~mm}$ in the endcap). The track resolution in the TRT is $130 \mu \mathrm{m}$ per straw. A typical track will generate 30 hits in the TRT. There are approximately 351,000 TRT channels.

\subsubsection{Calorimeters}

The ATLAS calorimeter, highlighted in Figure 2.6, is used to measure the energy of particles produced at the IP. It can be divided into three categories: the electromagnetic, hadronic, and forward calorimeters. A detailed discussion on calorimetry is provided in Chapter 3. Together, the ATLAS calorimeter systems provides coverage up to $|\eta|=4.9$, and has adequate longitudinal and transverse depth to contain the highest energy particles expected in ATLAS (except muons that only minimally ionize the material of the calorimeter).

\section{Electromagnetic Calorimeter}

The electromagnetic sampling calorimeter is used to measure the energy of electrons and photons, and provide initial energy measurements of more penetrating particles that pass through. It is made of liquid argon as the active material and lead as the absorber. The alternating layers of liquid argon and lead are accordion shaped; so uniform $\phi$ coverage is attained without cracks in $\theta$. It is separated into barrel and endcap pieces, each in separate cryostats.

The barrel electromagnetic calorimeter $(|\eta|<1.475)$ itself is separated into two half-barrels, with a separation of $4 \mathrm{~mm}$ at $|\eta|=0$. The electromagnetic barrel 


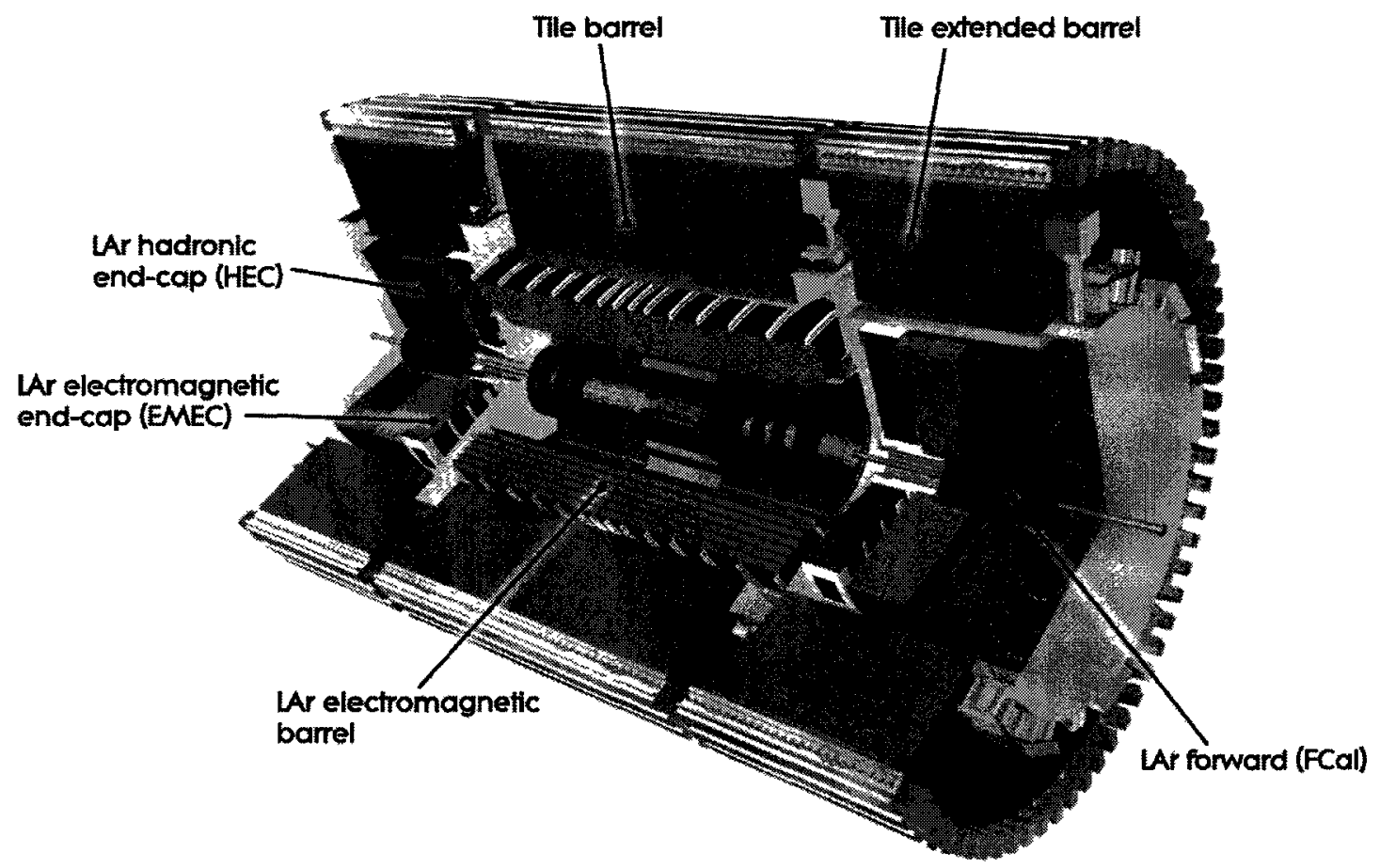

Figure 2.6: Layout of the calorimeter systems in ATLAS. The calorimeters that use liquid argon as the active material have the abbreviation LAr before their names.

calorimeter is made of three longitudinal sections called layer 1,2 , and 3 . The granularity and structure of these layers is shown in Figure 2.7. The liquid argon gap of the accordion structure is maintained at a constant size of $4.2 \mathrm{~mm}$, with an electrode positioned at the center of the gap to collect the electrons created via ionization by charged particles passing through the liquid argon. The first layer has a granularity of $\Delta \eta \times \Delta \phi=0.025 / 8 \times 0.1 \mathrm{rad}($ for $|\eta|<1.4$ ) and assists in the separation of $\pi^{o} \rightarrow \gamma \gamma$ and single photons. The second layer is the longest, and contains most of the electromagnetic shower generated by electrons or photons. The final layer is used to account for energy leakage from high energy electrons or photons not fully contained in the electromagnetic calorimeter.

The electromagnetic endcap calorimeter (EMEC) is located between $1.375<|\eta|<$ 3.2 in two endcap cryostats (one on each endcap side). For $1.5<|\eta|<2.5$ the EMEC 


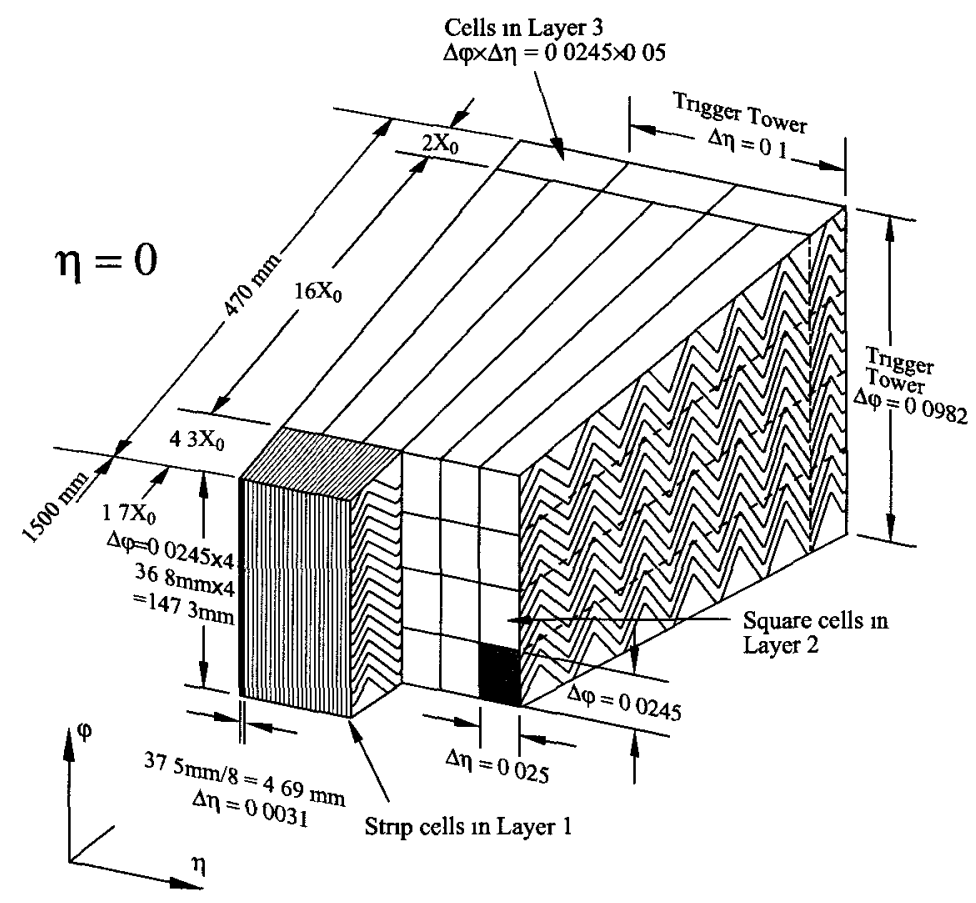

Figure 2.7: The liquid argon barrel electromagnetic calorimeter. The three layers $(1,2$, and 3 ) are visible, along with the channel granularity in $\eta-\phi$ for each.

is split into three longitudinal compartments, with the last one having granularity twice as large as the first two. The other regions of the EMEC, have two longitudinal layers and reduced granularity.

In front of the electromagnetic calorimeters $(|\eta|<1.8)$ a presampler of instrumented liquid argon is present. It is used to determine the amount of energy lost in upstream material before the calorimeters. The amount of upstream material (in radiation length $X_{o}$ ) as a function of $|\eta|$ in front of the presampler and accordion electromagnetic calorimeters is shown in Figure 2.8. A majority of the upstream material stems from the inner detector and the cryostat and their associated services. The region around $|\eta|=1.5$ is the crack between the barrel and endcap cryostats, and has the most amount of material in front of the calorimeter. Upstream material does degrade the energy resolution when the associated energy loss is not properly taken into account. 


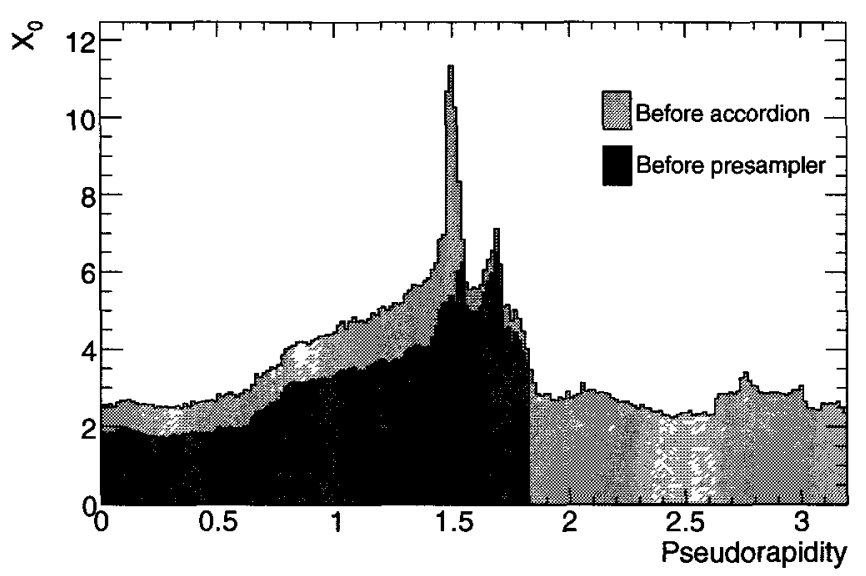

Figure 2.8: Amount of material upstream, in radiation lengths $X_{o}$, as a function of $|\eta|$ in front of the presampler and electromagnetic barrel calorimeters.

There are a combined total of approximately 173,000 readout channels between the barrel electromagnetic calorimeter, EMEC, and presampler. The overall energy resolution of the electromagnetic calorimeter is required to be:

$$
\frac{\sigma_{E}}{E}=\frac{10 \%}{\sqrt{E}} \oplus 0.7 \%
$$

where $\oplus$ means the terms are added in quadrature.

\section{Hadronic Calorimeter}

The hadronic calorimeter surrounds the electromagnetic calorimeter, and is used to measure the energy of hadron particles. The barrel component of the hadronic calorimeter is made of alternating scintillating tile and steel, as can be seen in Figure 2.9. Each tile is $3 \mathrm{~mm}$ thick and has a radial length that varies from $97 \mathrm{~mm}$ to $187 \mathrm{~mm}$ and an azimuthal size of $200 \mathrm{~mm}$ to $400 \mathrm{~mm}$. The tiles are assembled in wedge shaped modules similar to Figure 2.9 such that the tiles lie in the $R-\phi$ plane. There are 64 modules that form a cylinder around the electromagnetic cryostat and cover the region of $|\eta|<1.7$. It has a central barrel $5.8 \mathrm{~m}$ in length $(|\eta|<1.0)$. In 


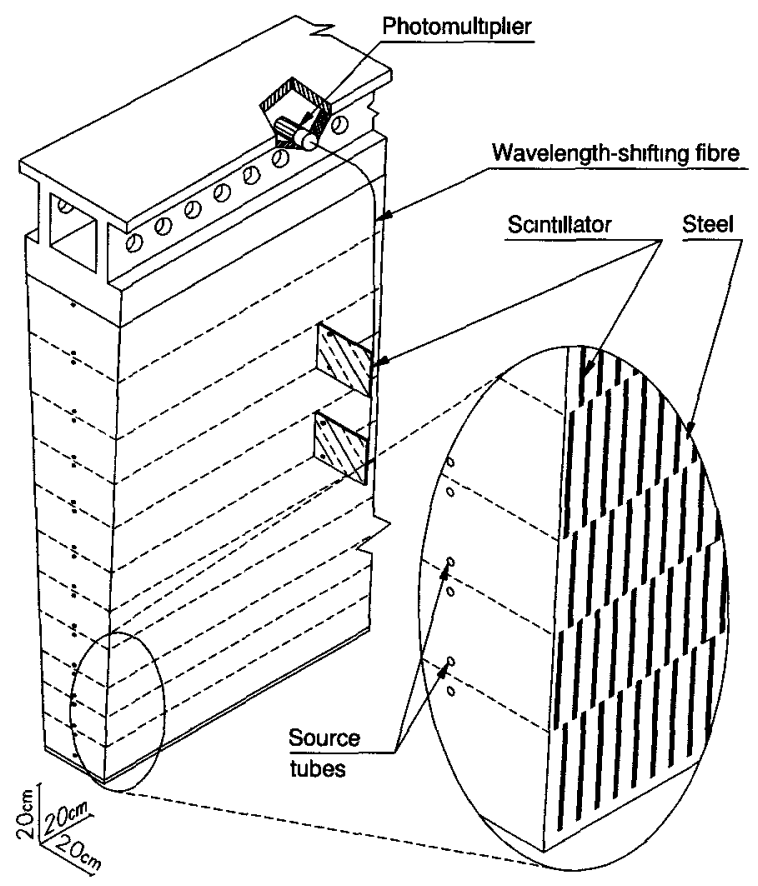

Figure 2.9: A module of the tile calorimeter showing the scintillating tile and steel structure, the wavelength shifting fibre and the photomultiplier tubes.

addition there are two extended barrels covering $0.8<|\eta|<1.7$, each $2.6 \mathrm{~m}$ in length. The gap of $0.6 \mathrm{~m}$ between the barrel and extended barrel is used for services and cables to the inner detector and liquid argon detectors, and has limited tile coverage. The scintillation light produced by charged particles in the scintillator is collected on either side of the tile by a wavelength shifting fibre that is read out by photomultiplier tubes.

In the endcap, the hadronic calorimeter (HEC) provides coverage in the range of $1.5<|\eta|<3.2$. It is positioned behind the EMEC in the same cryostat. The HEC is a sampling calorimeter made of copper and liquid argon. Unlike the electromagnetic calorimeters the alternating layers are flat, not accordion shaped. There are 32 wedgeshaped modules that form a cylinder concentric with the beam line.

The tile calorimeter and HEC systems are required to obtain an energy resolution 
of:

$$
\frac{\sigma_{E}}{E}=\frac{50 \%}{\sqrt{E}} \oplus 3 \% \text {. }
$$

\section{Forward Calorimeter}

The ATLAS forward calorimeter (FCal) is a sampling liquid argon calorimeter located in the same endcap cryostat as the EMEC and HEC. Figure 2.10 shows the endcap cryostat, and position of these three calorimeter technologies. The FCal spans a range of $3.1<|\eta|<4.9$, and has three longitudinal layers. The first layer, FCal1, is the first calorimeter traversed by particles travelling from the IP in this $\eta$ range. It is an electromagnetic calorimeter made of copper as the absorbing material. The second and third longitudinal layers called FCal2 and FCal3, are the hadronic calorimeter layers, and use tungsten as the absorbing material. Chapter 3 describes the calibration and performance of the FCal with test beam data. In that same chapter, Section 3.4 gives a detailed description of the FCal.

\subsubsection{Muon Spectrometer}

At the outer-most edge of the ATLAS detector sits the muon system. The muon spectrometer system is made of two systems, one for muon track reconstruction and the other for triggering. A schematic of the muon system (and the barrel toroid magnets for reference) is shown in Figure 2.11. For $|\eta|<2.0$ muon track reconstruction is done by Monitored Drift Tubes (MDTs), and for $2.0<|\eta|<2.7$ by Cathode Strip Chambers (CSCs). The MDTs are made with $30 \mathrm{~mm}$ diameter gaseous aluminum tubes. The CSCs are drift chambers and are better suited for the high flux of particles in the forward region. At $|\eta|=0$ there is a $30 \mathrm{~cm}$ gap in the coverage to allow essential services into the detector.

In the barrel $(|\eta|<1.4)$ the muon chambers lie parallel to the beam axis and these 


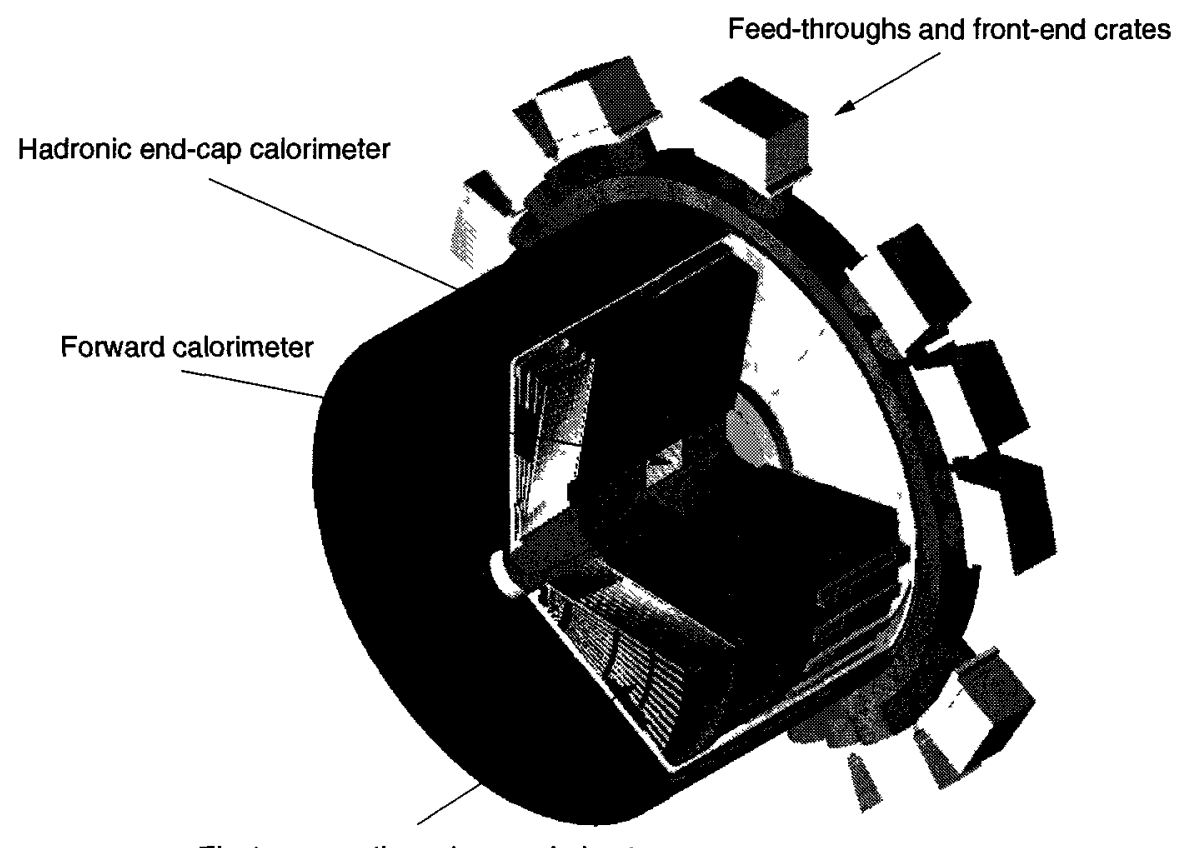

Electromagnetic end-cap calorimeter

Figure 2.10: The liquid argon endcap calorimeter, showing the cryostat containing the EMEC, HEC, and FCal. The interaction point is to the bottom-left of the figure.

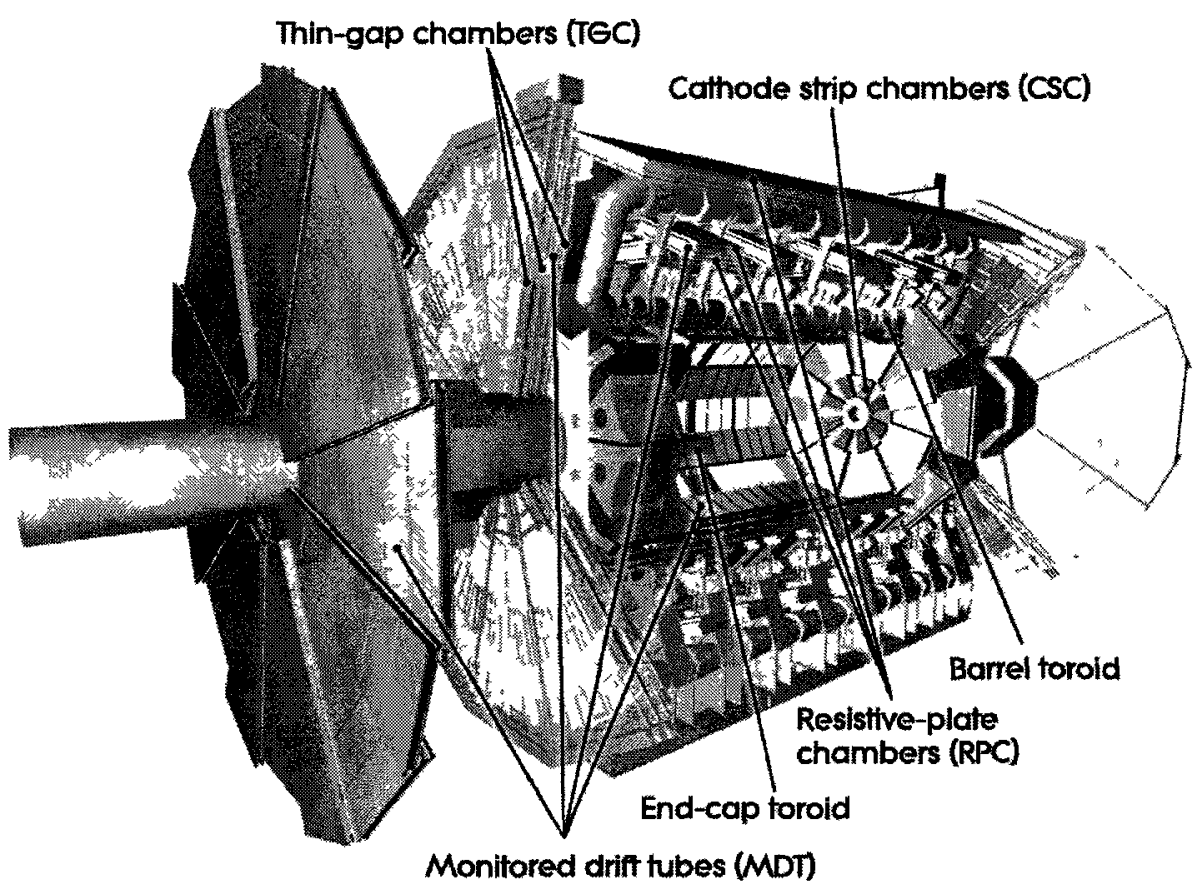

Figure 2.11: The components of the muon tracking reconstruction (MDT and CSC) and trigger (RPC and TGC) system. 
chambers are positioned inside and outside the barrel toroid at radii of $5 \mathrm{~m}, 7.5 \mathrm{~m}$, and $10 \mathrm{~m}$. Each chamber typically has three or four drift tube layers. In each endcap the muon spectrometer layers are assembled in wheels, at $|z|=7.4 \mathrm{~m}, 10.8 \mathrm{~m}, 14 \mathrm{~m}$, and $21.5 \mathrm{~m}$, on either side of the endcap toroid. In the endcap the chambers are perpendicular to the beam axis. The muon spectrometer is required to reconstruct muon momenta in the transverse plane with a resolution of $10 \%$ at $1 \mathrm{TeV}$.

For triggering the muon system uses Resistive Plate Chambers (RPCs) and Thin Gap Chambers (TGCs). The muon trigger system will be discussed in more detail in Chapter 4.

\subsection{Software for Analysis}

The software used in this thesis was developed using the ATLAS Athena framework [8], the CERN ROOT libraries [9], the g++ compiler and associated libraries.

Athena is the primary framework used in ATLAS for processing data and Monte Carlo (MC). It is used to interface MC generators (such as Pythia), detector simulation software (such as GEANT4), and analysis tools in a common framework. The software is primarily written in $\mathrm{C}++$, and the users can modify certain predefined configurable options via scripts in the Python programming language. The versions of Athena used in this thesis are 13.0.40 for the test beam data analysis, version 15.2.0 for the test beam MC analysis, and 15.6.9 for the top physics MC analysis.

The CERN ROOT framework was used to generate the plots in this thesis, and fit the data to user defined functions. The software was also used to apply and study analysis cuts. For the test beam analysis ROOT 5.14 .00 was used to produce the final plots. For the top MC analysis the version was ROOT 5.18.00.

For the test beam analysis, the raw data files were locally available at Carleton 
University. The Carleton University computing cluster was used to run the data analysis, and was used for the simulation, digitization, and reconstruction of the test beam MC.

The top quark cross-section analysis used as input MC samples that were produced by the ATLAS collaboration, in a format called analysis object data (AOD). These AOD files contain limited information about individual detector readout channels, however they do contain information about the final reconstructed physics objects, such as reconstructed electrons and muons. Information from AODs are accessed through Athena.

For the top quark cross-section analysis, small test jobs were run on the local Carleton University computing cluster, but the full statistics were analyzed using the LHC computing grid (LCG) [10], submitted via the ATLAS PanDA [11] interface. This system of running mass jobs remotely on the LCG follows the computing scheme encouraged by ATLAS management. Once the analysis was completed on the remote grid computing sites, the final files in the format of ROOT files were downloaded to the local Carleton University computers using the ATLAS Don Quijote 2 tools [12] (which allows users to view and download data and MC available on the LCG). 


\section{Chapter 3}

\section{Calibration of the ATLAS Forward}

\section{Calorimeter}

Particle detectors employ many different technologies to maximize the information about the outgoing flux of particles resulting from particle collisions. Systems of tracking detectors, magnets, and calorimeters are used to gain valuable information about each particle, such as charge, momentum, energy, and spatial location. This section describes how particles interact with matter and how this understanding is exploited in calorimetry. The basic function, design, calibration and goals of calorimeter detectors, in the context of ATLAS are described. This chapter presents the results of an analysis using FCal test beam data to find the intrinsic response and resolution of the FCal to electrons and hadrons. The data were analyzed with test beam specific and ATLAS reconstruction algorithms, with the focus on optimizing the latter. Comparisons of shower and detector properties are made to MC simulations. Finally the test beam data and MC are used to study the response at large values of $|\eta|$, near the inner edge of the FCal. 


\subsection{Particle Interactions in Matter}

The detection of particles exploits the manner in which particles interact with matter. A single particle can undergo many interactions, depending on its type and energy, and the medium that is traversed. The interactions can be via inelastic or elastic collisions, ultimately leading to energy loss of the incoming particle into the medium, and/or an altered trajectory of the incoming particle. Section 3.1 .1 will describe the general case of massive particles interacting with matter. The following Section, 3.1.2, describes the specific case of electrons, which experience additional dominant interactions due to their light mass. And the final Section, 3.1.3, details the various interactions photons can have with matter.

\subsubsection{Massive Particle Interactions in Matter}

The electromagnetic interaction is the main energy loss mechanism for massive charged particles with matter. As a charged particle enters a medium it encounters the electric field of the atomic electrons and nucleons. This results in the transfer of energy from the incoming particle to the atomic electrons, yielding ionization or excitation of the atoms in the medium.

For an incoming charged particle this energy loss $d E$ per unit length $d x$ can, on average, be approximated as [13]:

$$
-\frac{d E}{d x}=2 \pi N_{a} r_{e}^{2} m_{e} c^{2} \rho \frac{Z}{A} \frac{z^{2}}{\beta^{2}}\left[\ln \left(\frac{2 m_{e} \gamma^{2} v^{2} W_{\max }}{I^{2}}\right)-2 \beta^{2}-\delta-2 \frac{C}{Z}\right]
$$

with the definition for the variables given in Table 3.1.

Equation 3.1 is known as the Bethe-Bloch equation. The negative sign indicates the incoming particle is experiencing an energy loss per unit of distance. For high 


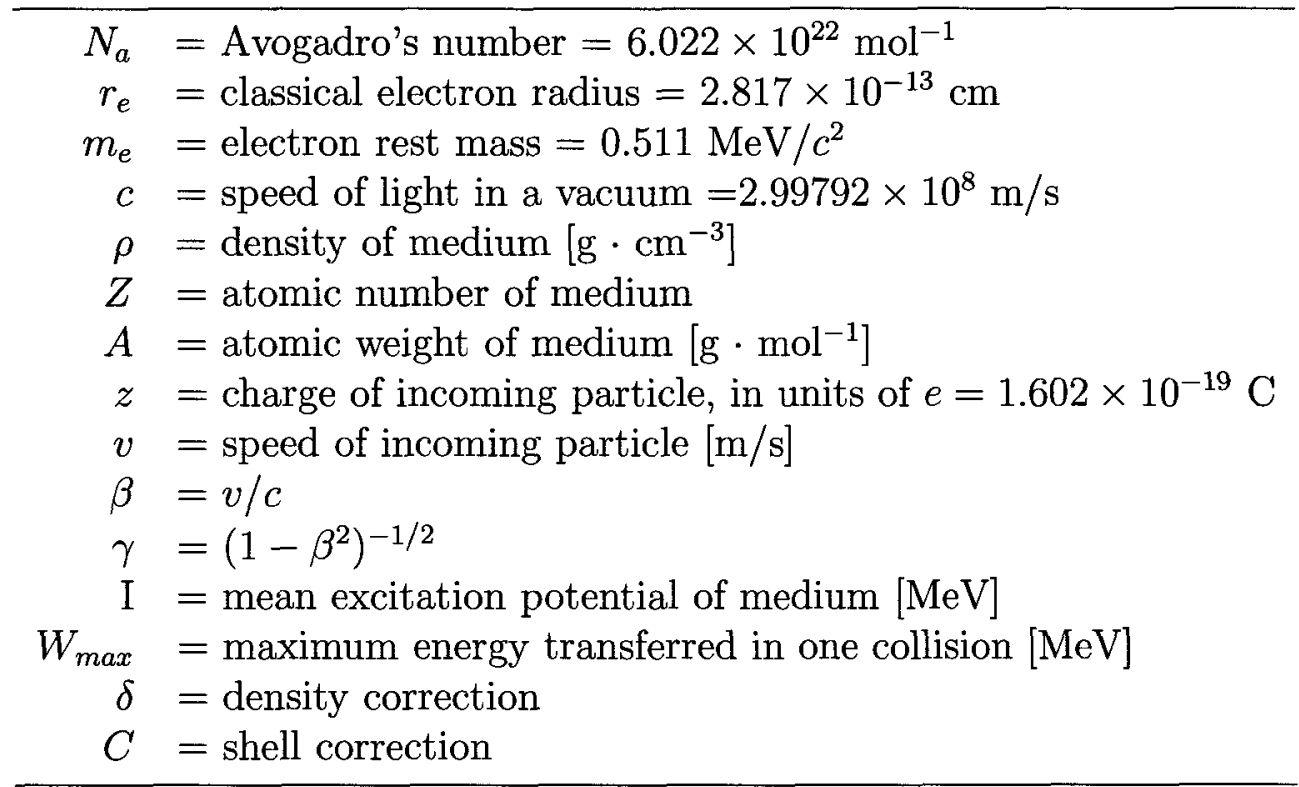

Table 3.1: The definition of the variables in the Bethe-Bloch equation for energy loss of massive charged particles in matter.

energies $(v \approx c)$ the amount of energy loss of the incident particle is dominated by the logarithm, and for low energies by the $\beta^{2}$ term. This equation is valid down to energies where $\beta \approx 0.1$. The density correction $(\delta)$ accounts for the screening of atomic electrons far from the incident particle trajectory due to polarization of atoms close to the path of the incoming particle. The shell correction $(C)$ applies to low energies, when the velocity of the incident particle is comparable to that of the (assumed stationary) orbiting bound electrons.

Neutral massive particles, such as neutral kaons, pions, and neutrons, do not experience energy loss in the material due to the electromagnetic force. Hadrons, both neutral and charged, can undergo nuclear interactions with the atomic nuclei. Short-lived unstable hadrons, such as the kaon, can decay in the material producing charged particles in the process. These massive charged particles can then lose energy by ionization, as described previously. 
Neutrons are a special case of a massive neutral particle, because of their much longer lifetime they are more likely to undergo a nuclear interaction with the material than decay via the weak force. The short-range strong force requires that the neutron pass close to a nucleus for the interaction to occur, hence neutrons can travel within a material for a longer distance than other massive particles. High energy neutrons $(>150 \mathrm{MeV})$ are likely to produce spallation interactions, striking a nucleus and producing secondary hadrons. Low energy neutrons can also be captured by the nucleus, which results in the release of gamma radiation, which will then interact with the medium. Photon interactions with matter will be described in Section 3.1.3.

\subsubsection{Electron Interactions in Matter}

Electrons and positrons are the lightest charged particles that interact predominantly in material via the electromagnetic force. The behavior of electrons and positrons as they lose energy in matter is similar, so unless otherwise stated the reactions described below for electrons also apply to positrons. Figure 3.1 shows the fraction of energy loss in lead for different processes as a function of the incoming electron energy for lead material. The general description for each of these interactions will be described, and is similar for all materials.

At low incoming electron energies the energy loss is dominated by ionization, as with massive charged particles. Equation 3.1 is slighty altered in the case of electrons to account for their light mass and the fact that there are identical particles in the initial and final states. The ionization energy loss is different for positrons, due to the slightly different cross-section of a positron (versus electron) to interact with an electron.

For high energy incident electrons $(E>100 \mathrm{MeV})$ the dominant source of energy 


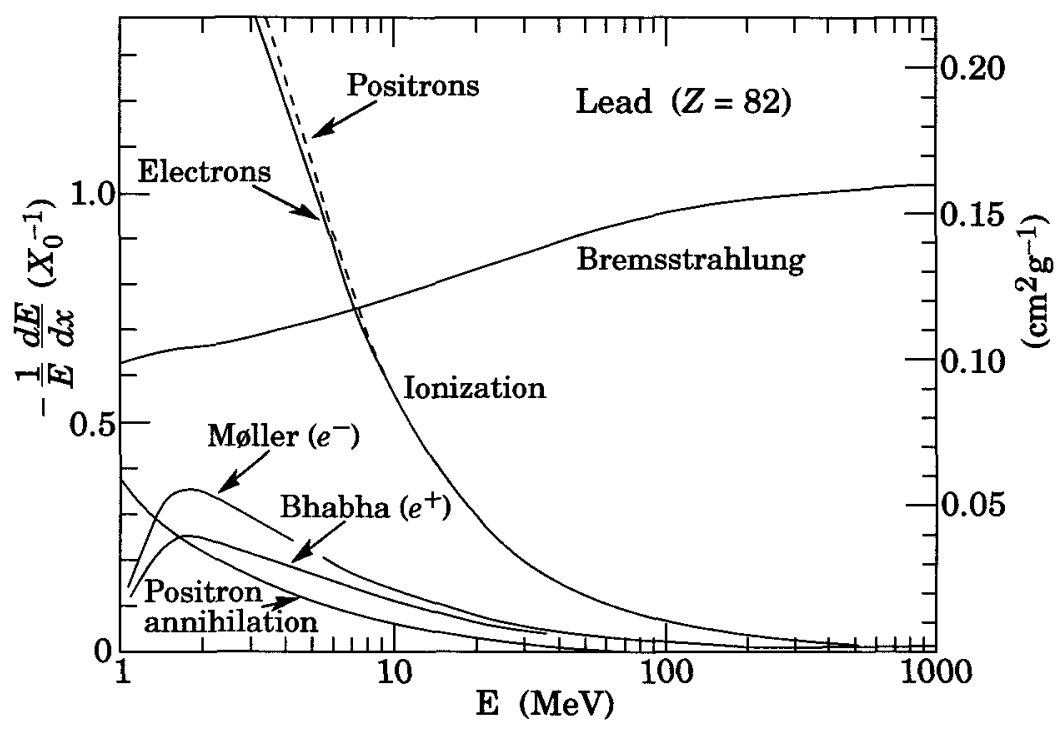

Figure 3.1: Typical electron and positron energy loss mechanisms for a range of incident electron energies in lead, from [14]. The $x$-axis shows the energy of the electron. The $y$-axis on the left shows the energy loss per unit length divided by the energy.

loss is Bremsstrahlung radiation. As the electron travels through the medium its straight-line trajectory is altered due to electromagnetic interactions with the material. This acceleration results in the release of 'breaking' radiation, the same as synchrotron radiation produced by electrons traveling in a circular orbit. The crosssection for an incoming particle of mass $M$ to produce a Bremsstrahlung photon is proportional to $1 / M^{2}$, so this type of energy loss is significant for electrons compared to heavier hadrons. The production angle of the photon with respect to the electron trajectory is inversely proportional to the initial energy of the electron. So at high energies, where this process dominates, photons tend to be produced along the direction of the electron path. There is a large variation in the possible fraction of initial energy the photon can carry away.

The energy at which ionization equals that of Bremsstrahlung is called the critical energy:

$$
\left(\frac{d E}{d X}\right)_{\text {brem. }}=\left(\frac{d E}{d X}\right)_{\text {ioniz. }}
$$


The critical energy $\left(E_{c}\right)$ for an absorbing material with atomic number $Z$ can be approximated as [1]:

$$
\begin{aligned}
& E_{c}=\frac{(610 \mathrm{MeV})}{(Z+1.24)} \quad \text { (solids and liquids) } \\
& E_{c}=\frac{(710 \mathrm{MeV})}{(Z+0.92)} \quad \text { (gases). }
\end{aligned}
$$

Substituting the atomic number for solid lead with $Z=82$, predicts the critical energy to be $7.3 \mathrm{MeV}$, which is approximately the cross-over point shown in Figure 3.1. The critical energy is an important concept when describing the cascade of particles that can result from these basic interactions, which will be detailed in Section 3.2

A measure of depth traversed by an electron in a medium is commonly called the radiation length $X_{o}$, and is defined as the thickness of the absorbing material necessary to reduce the average energy of high energy electrons (via radiation only) by a factor of $1 / e$. It reflects the electron density in the absorbing material and hence is a good description of the ability for the electron to interact via the electromagnetic force with the material.

As an electron travels through matter it interacts as described above, the net effect of these inelastic and elastic collisions on the incoming electron are continuous losses of energy and leads to a jagged trajectory.

\subsubsection{Photon Interactions in Matter}

Photons are neutral massless particles that can interact with matter via the electromagnetic interaction. An example of the various photon energy loss processes in matter as a function of photon energy is shown in Figure 3.2, in this case for a lead absorber. 


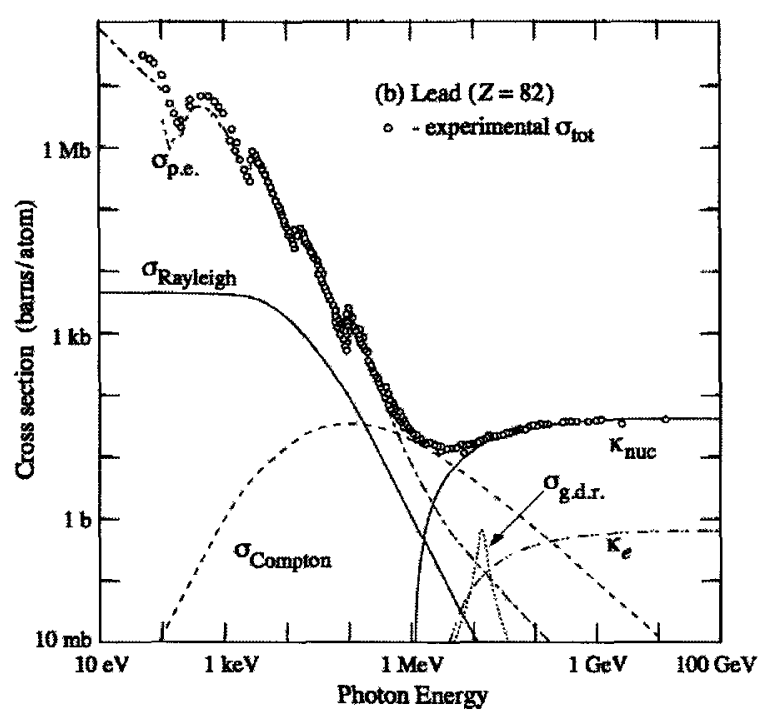

Figure 3.2: Typical photon energy loss methods for a range of incident photon energies plotted along the $x$-axis, taken from [1]. In this figure $\sigma_{\text {p.e. }}$ is the cross-section for the photoelectric effect, $\sigma_{\text {Rayleigh }}$ is for coherent scattering off an atomic electron, $\sigma_{\text {Compton }}$ is the cross-section for Compton scattering, $\kappa_{\text {nuc }}$ and $\kappa_{e}$ are the cross-sections for pair production in the presence of an atomic nuclear and atomic electron, and finally $\sigma_{\text {g.d.r }}$ is the cross-section for breaking up a nucleus. The dashed line represents the net theoretical cross-section summed over all processes and the open dots correspond to the experimental results.

For photons with $E<500 \mathrm{keV}$ the leading process for energy loss is the photoelectric effect; the process by which a photon is absorbed by an atomic electron which is then liberated from its bound state. Excess energy greater than the electron binding energy becomes the kinetic energy of the released electron. The cross-section is proportional to $1 / E^{3}[15]$, so it is dominant at low photon energies.

For intermediate photon energies $(500 \mathrm{keV}-50 \mathrm{MeV})$ the photon tends to lose most of its energy by Compton scattering off electrons in the medium. The incoming photon interacts with an atomic electron, transferring some of its energy to the electron. The recoil electron carries away some of the initial fraction of the photon's energy, and the photon is scattered and continues on an altered path.

At high energies $(E>50 \mathrm{MeV})$ the photon energy loss is dominated by pair production. Pair production is independent of photon energy, but there is a minimum 
requirement that there is enough energy to produce the particle pairs. The remaining energy is shared between the pair, but is not required to be shared equally. The production angle varies inversely with photon energy, so at high energies the pair are produced in the same direction as the photon trajectory. The electron and positron pair will then interact with matter and lose their energy as described in the previous section. The mean distance a photon travels before conversion into an electronpositron pair is $1.3 X_{o}$. The process of energy loss by an incoming particle into the production of secondary particles initiates what is called a particle shower.

\subsection{Particle Showers}

Consider a particle entering a medium with some initial energy $E_{o}$. Via the electromagnetic, strong, and weak forces, this energy can be sequentially transformed into different forms, creating new particles and transmitting energy to existing particles that themselves can produce secondary particles. Although one particle enters the medium, through its interactions with the material it can set off a chain reaction producing new particles and excited states. There are two types of particle cascades: electromagnetic shower and hadronic shower. The distinguishing trait is the type of particle initiating the cascade. Electromagnetic showers are initiated by electrons, positrons, and photons; whereas hadron showers are initiated by hadrons or gluons.

\subsubsection{Electromagnetic Showers}

When photons, electrons, and positrons interact with material a number of lower energy particles are produced. In turn, these secondary particles interact electromagnetically with each other and the material, yielding a cascade of electromagnetic particles, creating an electromagnetic shower. 
The dominant interaction for a high energy electron in matter is an interaction with an atomic electron, radiating a Bremsstrahlung photon. If the photon has sufficient energy after some depth it will interact with the medium to produce an electron-positron pair, which themselves can then continue the cycle producing Bremsstrahlung photons. The energy in the shower is split amongst its constituents, so as the number of particles grows the average energy of each particle decreases. The shower will continue to grow until a) the remaining electrons have lost sufficient energy and reached their critical energy at which point ionization will dominate the energy loss, and b) photons no longer have sufficient energy to pair produce and energy loss mechanisms such as Compton scattering and the photoelectric effect take over. In the final stage the low energy electrons and photons undergo interactions which no longer produce new particles.

A simple model of electromagnetic shower propagation is shown in Figure 3.3. A photon enters a medium from the left, with some initial energy $E_{o}$. It is assumed that after one radiation length $X_{o}$ the photon will produce an electron-positron pair. The electron and positron will split the energy of the photon equally, each having energy $E_{o} / 2$. Each lepton then produces a Bremsstrahlung photon after they have travelled a distance $X_{o}$. The lepton and Bremsstrahlung photon will each have half of the lepton's initial energy. And so on.

Using this simple model one can estimate the number of particles $N$ in the shower after $t$ radiation lengths:

$$
N(t)=2^{t}=e^{t \ln 2} .
$$

The simple model assumes there are equal numbers of photons, electrons, and positrons. Assuming the energy is always split equally between the final state particles at each 


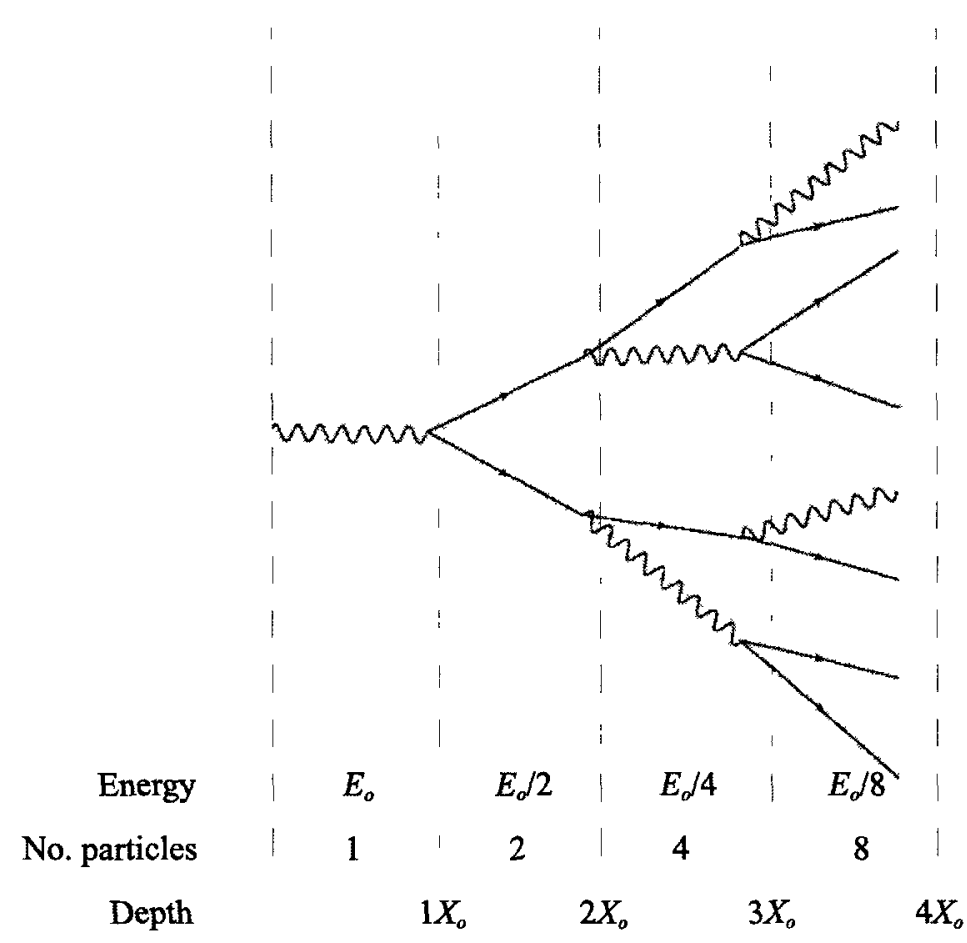

Figure 3.3: A diagram of simple electromagnetic shower development starting from an incoming photon with initial energy $E_{o}$ entering some material from the left. The simple model assumes a photon will pair produce after one radiation length, $X_{o}$, and electrons will produce a Bremsstrahlung photon after one $X_{o}$. The model assumes that at each interaction the energy is split equally between the final particles. The multiplicity of particles and energy of each particle at each radiation length is also shown.

interaction, the average energy $E$ of each particle after $t$ radiation lengths is:

$$
E(t)=\frac{E_{o}}{2^{t}}
$$

where $E_{o}$ is the initial energy of the incoming particle. The shower maximum will occur when there is the maximum number of particles in the shower. This happens at an energy of $E=E_{c}$, and corresponds to a number of radiation lengths:

$$
t_{\max }=\frac{\ln \left(E_{o} / E_{c}\right)}{\ln 2} \quad\left[X_{o}\right]
$$


Thus the longitudinal depth of the shower depends on the energy of the incoming particle and the material (which sets the value of $E_{c}$ ). The number of particles produced is proportional to the energy of the incoming particle. The higher the energy, the more secondary particles it will produce that will have sufficient energy to continue the shower development. A higher energy shower will penetrate more deeply into the material.

The above is a very simplistic model that does not take into account the fluctuations in shower development and the more complex processes at lower energy. The model is an approximation that deals with averages. Comparison with data indicate a better description of the longitudinal depth where the maximum number of particles are produced in an electromagnetic shower is [5]:

$$
t_{\max }=3.9+\ln E_{o} \quad\left[X_{o}\right],
$$

with $E_{o}$ in units of $\mathrm{GeV}$ for incoming particles with energies in the range of $2 \mathrm{GeV}$ to $300 \mathrm{GeV}$.

A shower will spread in the radial direction due to multiple scattering and pair production. In a more realistic approximation of lateral shower development, a cylinder with a radius equal to the Moliére radius contains $\sim 90 \%$ of the electromagnetic shower, where the Moliére radius $R_{M}$ is defined as:

$$
R_{M}=21 \frac{X_{o}}{E_{c}} \quad\left[X_{o}\right]
$$

where $E_{c}$ is the critical energy in $\mathrm{MeV}$.

In practical applications, shower development is predicted by complex MC algorithms that make use of the actual cross-sections for each process and particle type, 
for a given incoming particle energy and medium.

\subsubsection{Hadronic Showers}

The second classification of particle showers are hadronic showers, initiated by incoming hadrons undergoing a nuclear interaction with the atomic nuclei.

The dominant source of energy loss for high energy, massive charged particles is ionization and excitation of the medium. Some of the incoming hadron's energy is converted into the production of secondary hadrons, which themselves carry away energy and repeat the process of hadron production, decay, and ionization, expanding the shower.

The initial interaction of the hadron with the material may produce $\pi^{o}$ particles that almost exclusively decay to some combination of photons, electrons, and positrons, initiating an electromagnetic component in the hadronic shower. The secondary hadrons can also interact to produce these electromagnetically decaying hadrons. The fraction of the electromagnetic component in the hadron shower depends on many parameters such as the material, the incoming energy, but on average approximately one third of the energy in a hadronic shower is used to produce particles that will decay electromagnetically [16].

Sometimes when unstable hadrons decay they produce muons. Muons mainly interact via the electromagnetic force, but have a small probability of producing Bremsstrahlung photons, hence losing very little energy in the material over large radiation lengths. Unstable hadrons can also produce neutrinos that only weakly interact and normally leave the material without interaction. These types of particles carry away some fraction of the initial energy of the incoming particle outside of the limited depth of the material. 
Energy can be transferred to the nucleus and used to break apart the nucleons if the energy is greater than the binding energy of the nucleus (on the order of a few MeV's), resulting in energy transfer to the nuclear fragments. Some of the original binding energy can be recovered when slow neutrons get captured by other nuclei but this release of energy from the recapturing can take microseconds to occur, which is on the order of a thousand times later than most interactions (possibly outside the window of detection).

These forms of energy loss (muons, neutrinos, nuclear fragmentation) are classified as invisible energy, and can be up to $30 \%$ of the initial particle's energy. The fraction of invisible energy decreases as incoming particle energy increases.

The mean free path of a hadron without interacting in matter is called the nuclear interaction length. It is defined as [5]:

$$
\lambda=\frac{A}{N_{A} \sigma_{\mathrm{abs}}},
$$

where $A$ is the atomic mass of the material, $N_{A}$ is Avagadro's number, and $\sigma_{\text {abs }}$ is the absorption cross-section for the particle to be absorbed by the material. Similar to the radiation length for electrons, it is a useful quantity for describing hadronic shower shapes in matter as it is approximately independent of material type.

For hadronic showers one approximation of the depth at which the maximum number of shower particles are present is [5]:

$$
\lambda_{\max }=0.90+0.36 \ln E_{o} \quad\left[\mathrm{~g} \mathrm{~cm}^{-2}\right],
$$

where $E_{o}$ is the initial particle energy in $\mathrm{GeV}$. As with electromagnetic showers, as the incoming particle energy increases the shower depth also increases. 
The short-range strong force requires that hadrons be close to the atomic nuclei for interactions to occur, which is a relatively small fraction of space occupied by the atom. Therefore hadrons can travel farther without interacting, hence hadronic showers tend to be larger in the longitudinal and transverse direction than electromagnetic showers. For an electron and pion of the same energy traversing the same material, the energy deposited per unit volume in the matter for the pion shower will be less than the electron's electromagnetic shower.

\subsection{Calorimeters for Particle Detection}

A detector commonly used for measuring particle energy is referred to as a calorimeter. Calorimeters work by completely absorbing the incoming particle and converting its energy into different forms that are used to create an electronic signal that can be detected. Calorimeters allow for the measurement of energy of both charged and neutral particles. In calorimeters it is highly desirable that the collected signal be proportional to the energy of the incoming particle for the range of particle energies under study. As the measured quantity for a calorimeter is energy, calorimeters are designed to have optimal energy resolution, which can be a challenge given the variations in showers development. In the design of a calorimeter other considerations are its ability to provide particle identification, position reconstruction, signal stability, and fast signal processing.

To fully capture the energy of an incoming particle the depth and width of a calorimeter should be sufficient to fully contain both the incoming particle and the secondary shower particles it produces. Electromagnetic calorimeters are designed to capture electromagnetic showers, and are described by their radiation length $X_{o}$;

typical electromagnetic calorimeters are 15-30 $X_{o}$ deep. Hadronic showers are more 
penetrating, so the depth of a hadronic calorimeter required for good containment is usually 5-10 $\lambda$. For example, a block of lead would need to be approximately $8.42 \mathrm{~cm}$ thick for $15 X_{o}$ (with a radiation length of $0.5612 \mathrm{~cm}$ ), and it would need to be 87.95 $\mathrm{cm}$ thick for $5 \lambda$ (with a nuclear interaction length of $17.59 \mathrm{~cm}$ ). For lead, $\lambda=31.3$ $X_{o}$, demonstrating that hadronic calorimeters must be significantly thicker or made of denser material to absorb the energy of the incoming particle.

A large fraction (20-30\%) of energy in hadronic showers is invisible (not recorded by the detector). In non-compensating calorimeters the invisible energy is not recovered so the response to incoming electrons and hadrons of equal energy is not equal, with the electron having a larger amount of its energy detected. The fraction of the electromagnetic shower component in a hadronic shower depends on the energy of the incident hadron (in general more $\pi^{o}$ 's are produced at higher energy), and often hadronic calorimeters suffer from a non-linearity in the signal response as one goes from low energies (100's MeV) to high energies (100's GeV).

Calorimeters that measure the amount of ionization measure the number of ion pairs produced, which is proportional to the incoming particle energy. The statistical uncertainty on the number of ion pairs $(N)$ can be approximated on average using Poisson statistics:

$$
\sigma(N)=\sqrt{N} .
$$

The energy resolution is defined as $\sigma(E) / E$, where $\sigma$ is the uncertainty associated with the measured energy $E$. In an ideal calorimeter $E \propto N$, so the uncertainty on the energy measurement is $\sqrt{E}$. Then the energy resolution can be written as:

$$
\frac{\sigma(E)}{E}=\frac{\sqrt{E}}{E}=\frac{1}{\sqrt{E}}
$$

where $E$ is the incoming particle energy. As the energy of the incoming particle 
increases the energy resolution of a calorimeter improves.

Calorimeters can be homogeneous, where the absorbing material is fully sensitive to the incident particle energy deposition. Alternatively, calorimeters can be of the sampling type, which have alternating layers of sensitive material and heavy absorbing material. To fully contain a shower originating from a high energy particle, homogeneous calorimeters must be quite large and can be costly due to the large amount of material necessary to contain the particle, so high energy cascades are typically detected by sampling calorimeters. In sampling calorimeters the active region is typically made of scintillators, nobel liquids or gases. The absorbing layer is typically a heavy (high $Z$ ) material such as copper, uranium, tungsten, or lead.

For sampling calorimeters, the energy resolution $\sigma(E) / E$ is parameterized in the following way:

$$
\frac{\sigma(E)}{E}=\frac{a}{\sqrt{E}} \oplus b \oplus \frac{c}{E}
$$

where $\oplus$ means the terms are added in quadrature. The variables $a, b$, and $c$ are constants that reflect the characteristics of a particular calorimeter. The variable $a$ is called the stochastic term (coming from $\sigma / E \propto 1 / \sqrt{E}$ ) and accounts for fluctuations in the number of ion pairs produced and the particle interactions in the shower development. Shower fluctuations in the calorimeter can be greater in the presence of material upstream, or inactive regions of the calorimeter like the absorber. Stochastic fluctuations in shower development are larger for hadronic showers, so the $a$-term tends to be larger in hadronic calorimeters. The fluctuations in a sampling calorimeter will be larger because sampling the shower is also a statistical procedure. The stochastic term dominates the energy resolution for low energy incident particles. The second term $b$ is a constant for all energies. It is the inherent systematic error of the detector, taking into account detector non-uniformities and mis-calibrations. It is 
the dominant term at high energies. The $c$-term represents the inherent fluctuations in the response due to the construction and instrumentation of the calorimeter, it is often referred to as the noise term. Lower electronic noise is desired because it will improve the overall energy resolution.

\subsection{The ATLAS Forward Calorimeter}

The physics program at the LHC has a strong emphasis on the discovery (or exclusion) of the SM Higgs boson. One way the Higgs boson is produced is in association with two forward jets from the initial interacting partons. A forward calorimeter makes a major contribution to identifying these forward jets.

There are many precision physics analyses, such as the top pair cross-section measurement, that rely on a good measurement of the energy deposited by particles. And there are many physics discovery modes, like the search for supersymmetry, that have the distinct signature of large missing energy which is derived from the sum of energy depositions by all particles. To achieve a good calculation of missing energy requires having a large calorimeter $|\eta|$ coverage, and calorimeters with good energy resolution and energy distributions that have small tails. These physics requirements have driven the design parameters of the ATLAS FCal.

The FCal is a sampling calorimeter located in the forward region, very close to the beam pipe, spanning $3.1<|\eta|<4.9$. It is cylindrical, concentric with the beam pipe, and separated into three longitudinal segments: FCal1, FCal2, and FCal3. Each longitudinal segment has a length of $444 \mathrm{~mm}$ along the $z$-axis. The inner radius of FCal1 is only $72.3 \mathrm{~mm}$ from the center of the beam line $(78.8 \mathrm{~mm}$ for FCal2, and 85.7 $\mathrm{mm}$ for FCal3), and the outer radius for all modules is approximately $450 \mathrm{~mm}$ [17]. The front face of FCal1 is the closest to the IP, at a distance of $|z|=4.7 \mathrm{~m}$. 
There are two sets of FCal modules, one on each side of the IP (referred to as side A and C). They are housed in the endcap cryostats, which also hold the EMEC and HEC calorimeters. The muon subsystem benefits having the FCal upstream because the FCal reduces the background seen by the muon detectors.

The forward region is subject to large amounts of radiation from minimum bias events. The beam pipe and FCal are both expected to activate and emit radiation. There will also be a high neutron flux in the FCal, approximately $105 \mathrm{kHz} / \mathrm{cm}^{2}$ for neutrons with energies greater than $100 \mathrm{keV}$ at the design luminosity of $10^{34} \mathrm{~cm}^{-2} \mathrm{~s}^{-1}$ [17].

It is expected that at the LHC design luminosity the FCal will be exposed to a maximum of $160 \mathrm{kGy}$ per year [7]. With this in mind, all materials composing the FCal must be radiation hard. The sensitive region of the FCal is filled with liquid argon, a radiation hard noble liquid, that will remain stable for the lifetime of the LHC (approximately 10 to 15 years). It is also a fast medium for the collection of ion pairs, which is important as the LHC nominal bunch crossings are spaced $25 \mathrm{~ns}$ apart. At standard pressure and room temperature argon is a gas, so maintaining argon in a liquid state requires the cryostat volume to be kept at approximately $90 \mathrm{~K}$.

The FCal is a sampling calorimeter and is made up of absorbing material (copper in FCal1, and tungsten in FCal2 and FCal3) and active liquid argon regions. The signal is collected with electrodes that have cylindrical geometry and run parallel to the beam pipe, for approximately the length of a given module. A schematic of the front of an electrode as seen by a particle coming from the IP is shown in Figure 3.4. The inner rod, made of copper (tungsten) in FCal1 (FCal2, FCal3), is kept at a positive potential. This rod is surrounded by a grounded tube made of copper (in all modules). The space between the rod and tube is filled with liquid argon. To maintain a constant liquid argon gap along the length of the electrode a radiation 


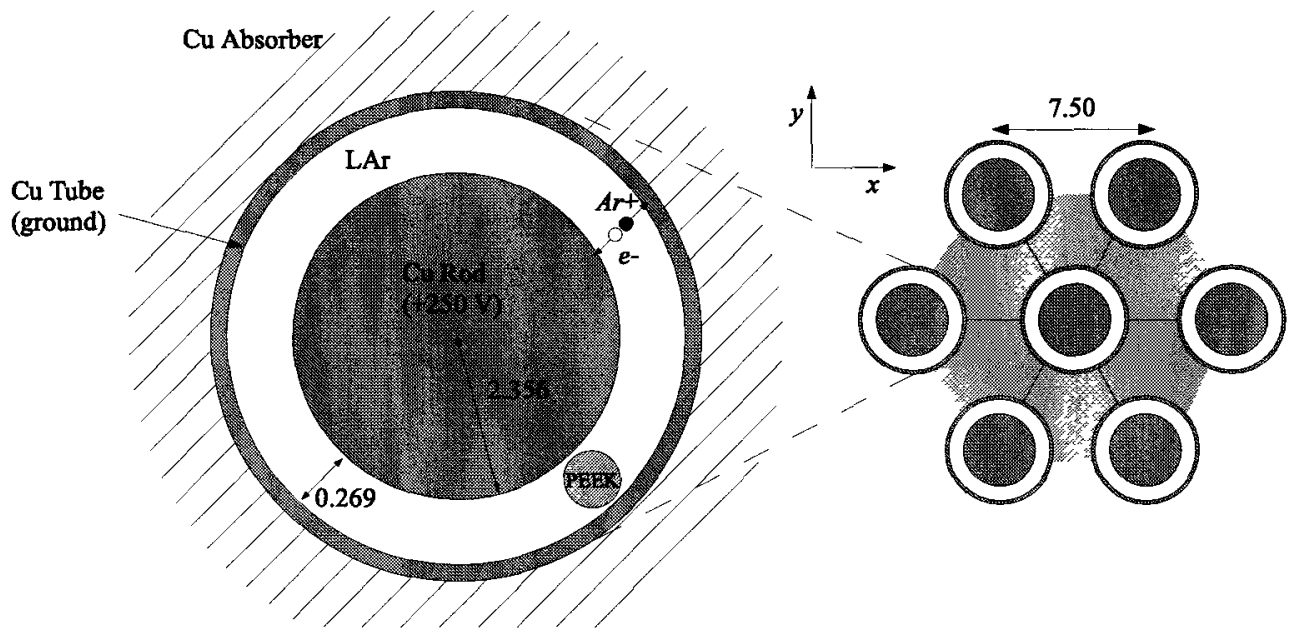

Figure 3.4: A schematic of an electrode in FCal1 is shown at the left. This forms one edge of the more general hexagonal electrode structure that is shown to the right. All units are in mm. LAr is liquid argon. Note the liquid argon gap is not drawn to scale, it is enlarged for qualitative purposes, the relative sizes are indicated on the diagrams.

hard fiber (PEEK) is helically wound around the rod.

One important and remarkable feature is the size of the liquid argon gap, which is only $269 \mu \mathrm{m}(376 \mu \mathrm{m}, 508 \mu \mathrm{m})$ in FCal1 (FCal2, FCal3). This gap size is significantly smaller than the standard liquid argon gaps in other calorimeters, which are typically on the order of millimeters. This gap size was chosen to reduce ion build up in the high radiation environment that could alter the drift time of the electrons to the rod, affecting the signal stability.

The electrode structure is repeated in the transverse direction in a hexagonal pattern with equal spacing between the centres of the electrodes, as seen to the right in Figure 3.4. In FCal1 the spacing between the electrode centre is $7.50 \mathrm{~mm}(8.18 \mathrm{~mm}$ in FCal2, and $9.00 \mathrm{~mm}$ in FCal3). Figure 3.5 is a schematic of the front face of the FCal1 looking head-on to the repeated electrode pattern. There are 12260,10200 , and 8224 electrodes in FCal1, FCal2, and FCal3, per endcap side, respectively.

The FCal1 is the first calorimeter seen by particles coming from the IP with 


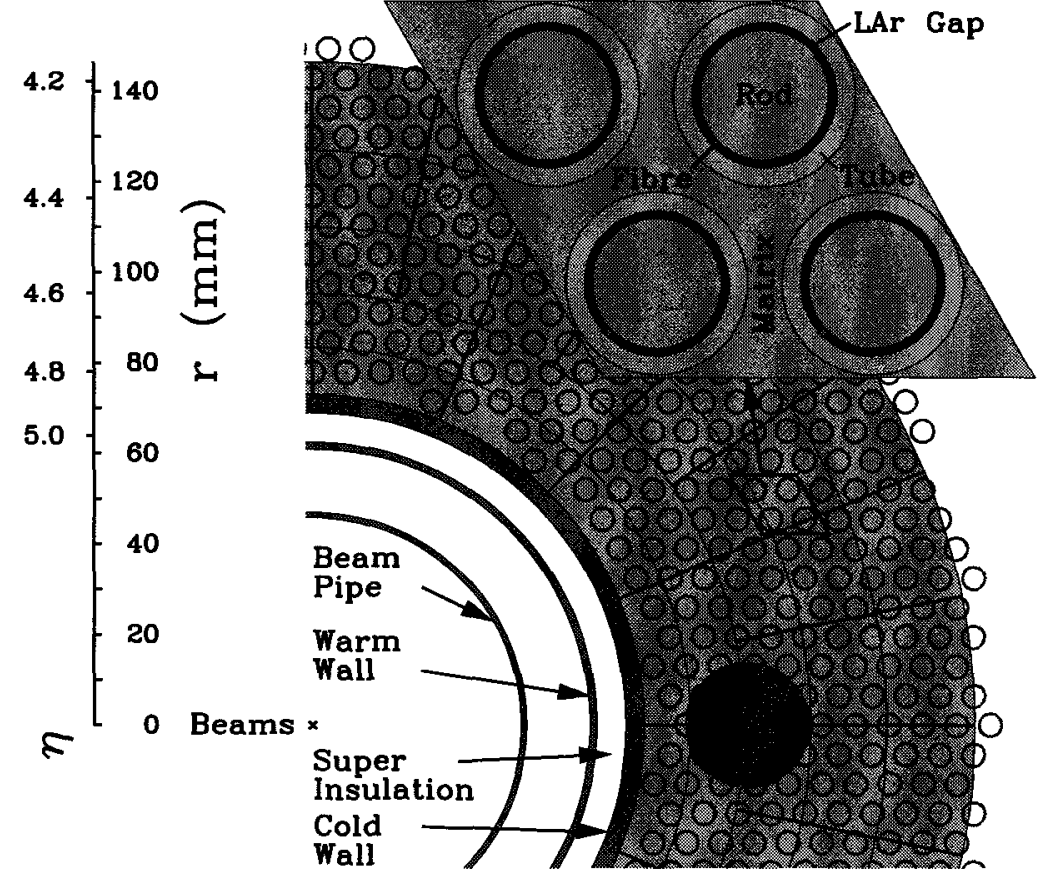

Figure 3.5: The front face of FCal1, with approximate spacings. The electrode structure can be seen, as well as a zoom of one electrode group containing four electrodes. The Moliére radius $\mathrm{R}_{\mathrm{M}}$ of FCal1(17.1 mm) is superimposed on the figure. At the inner edge the endcap cryostat wall and beam pipe can be seen. Figure taken from J. Rutherfoord.

$3.1<|\eta|<4.9$, and so it is classified as an electromagnetic calorimeter. Copper is the absorbing material in FCal1. Copper is the ideal absorber as it is a good conductor of the heat that arises from the large amounts of radiation. The Moliére radius of FCal1 is $17.1 \mathrm{~mm}$ [17] which is larger than the electrode spacing. So showers will on average spread over many electrodes which helps reduce any impact point dependence and improve the energy resolution. The total interaction length of FCal1 is $27.6 X_{o}$.

FCal2 and FCal3 are the hadronic compartments of the forward calorimeter and must be made of dense radiation hard material to fully contain the hadron showers in a longitudinal depth of only about $1.5 \mathrm{~m}$ (FCal1 + FCal2 + FCal3). Tungsten is the absorbing material that satisfies this criteria. The total nuclear interaction length of the FCal is $9.5 \lambda$. 


\subsubsection{Electronics Chain}

Ionization of the liquid argon combined with the electric field between the electrode rod and tube causes the electrons to drift towards the rod, giving rise to an electric pulse. The signal pulse is collected from each electrode by an interconnect board, as shown in Figure 3.6. On the interconnect board the signals from four (FCal1), six (FCal2), and nine (FCal3) electrodes are summed together, forming what is called an electrode group. The signal from an electrode group is carried to a summing board. For most of the FCal four interconnect boards are summed together and read out as a single channel (therefore most readout channels in FCall are composed of 16 electrodes). The exception to this are the interconnect boards at the inner and outer edges of the FCal, where the interconnect boards are not summed, due to geometry constraints and to provide the benefit of increased granularity in these transition regions.

The summing boards also provide the high voltage (HV) distributions to the electrodes along the coaxial cable. Each electrode group has its own HV supply. The four independent HV lines on each summing board ensure that in the event of a dead or faulty HV line only a fraction of the total signal collection is lost, but information about energy deposition from that geometrical region of the detector is still available. The signal from a summing board is carried by the feedthrough to the warm electronics and the front-end boards outside the cryostat via a coaxial 'pigtail' cable.

The signal passes to the front-end boards in the warm electronics, where it undergoes pre-amplification and signal shaping. The pulse entering the preamplifier is triangular in time, with a steep rise and a slow falling tail, where the full pulse shape spans approximately $65 \mathrm{~ns}$ in FCal1 (a reflection of the short drift time of electrons 


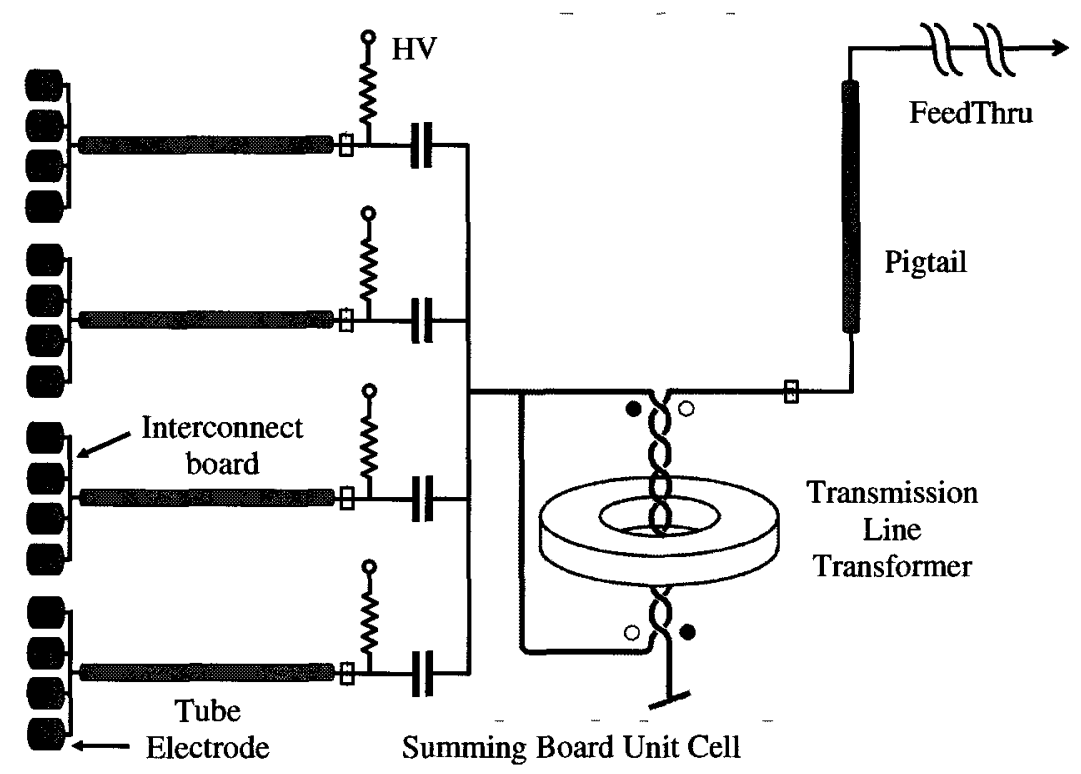

Figure 3.6: A schematic of the cold electronics located inside the cryostat for a typical FCal1 readout channel. The signal pulse from four (FCal1) electrodes is summed on the interconnect boards. For this typical channel, four such electrode groups are summed on the summing board. Note there are four independent HV lines on the summing board, one for each electrode group. Figure created by J. Rutherfoord and taken from [17].

across the liquid argon gap). The preamplifier maintains this shape and feeds it into the shaper. The shaper modifies this triangular pulse into a bipolar pulse, one that has a rounded peak followed by a rolling negative lobe. There are three gain settings in the shaper, low, medium and high, each with a gain factor of about 10 greater than the previous step. The pulse from each gain setting is sampled every $25 \mathrm{~ns}$ and the signal undergoes an analogue-to-digital converter (ADC), giving a number proportional to the height of the pulse at that time sample. The ADC value of the maximum sample is used to determine the final gain setting recorded for further analysis. The current choice is the highest gain setting that does not overflow the maximum ADC value, to maintain $\mathrm{ADC}$ linearity. The gain choice is made event-by-event and is 
called the autogain setting.

The final number of channels read out are 1008, 500, and 254 for FCal1, FCal2, and FCal3, respectively per side.

\subsubsection{Summary}

A summary of some properties for each FCal module is shown in Table 3.2. More information about the FCal can be found in [17].

\begin{tabular}{|r|c|c|c|}
\hline \hline Property & FCal1 & FCal2 & FCal3 \\
\hline Absorber material & $\mathrm{Cu}$ & $\mathrm{W}$ & $\mathrm{W}$ \\
Inner/outer radius [mm] & $72.3 / 449.4$ & $78.8 / 449.4$ & $85.7 / 449.4$ \\
Liquid argon gap [mm] & 0.269 & 0.367 & 0.508 \\
Depth [mm/ $\left.X_{o} / \lambda\right]$ & $444.1 / 27.6 / 2.65$ & $444.1 / 89.7 / 3.6$ & $444.1 / 88.0 / 3.55$ \\
Moliére radius [mm] & 17.1 & 11.9 & 12.1 \\
Electrode spacing [mm] & 7.50 & 8.18 & 9.00 \\
Number of electrodes (per endcap) & 12,260 & 10,200 & 8,224 \\
Number of channels (per endcap) & 1008 & 500 & 254 \\
\hline \hline
\end{tabular}

Table 3.2: A summary of some important properties of the forward calorimeter.

\subsection{Test Beam Introduction}

Calibration of any detector is a multi-step process involving prototypes, test beams, monitoring, and in-situ calibrations. These are done to extract calibration constants, to establish stability over time, and to test reconstruction techniques and compare the response of real data with that of the simulation.

A test beam was performed in the summer of 2003 to calibrate the FCal. It took place at CERN with one of the final set of FCal endcap modules (C-side) consisting of FCal1, FCal2, and FCal3. It was the final opportunity to test the modules directly 
with single particles before their installation into the endcap cryostat and prior to the cryostat being lowered into ATLAS. The specific goals of this test beam were to:

1. Measure the conversion between the signal response in ADC units and energy for electrons, setting the so-called electromagnetic scale of FCal1 (the response of FCal1 to electromagnetic showers). This was set using electron beams with a range of energies, and beams with minimal upstream material. The beams were directed into a location of the FCal that ensured full containment of the showers.

2. Measure the response of the detector at the electromagnetic scale across a variety of electron beam energies to test for a linear response.

3. Measure the electromagnetic and hadronic energy resolutions for a) the intrinsic detector and b) one with additional upstream material designed to represent ATLAS-like conditions.

4. Apply and study different reconstruction and hadronic weighting schemes to the reconstruction of energy in the FCal.

5. Examine the energy loss and showering behavior near the beam pipe (high $|\eta|$ ). This was done by using beams of particles impacting the calorimeter close to its inner edge.

6. Ensure that the response, as well as the electromagnetic and hadronic shower properties are correctly represented in the $\mathrm{MC}$ simulations. $\mathrm{MC}$ samples are studied to determine the signature and significance of many physics processes.

A test beam is the only time particles of known type and energy will be put into specific, known locations of the detector, so it is critical that the data be taken and analyzed to ensure the above goals are met. 


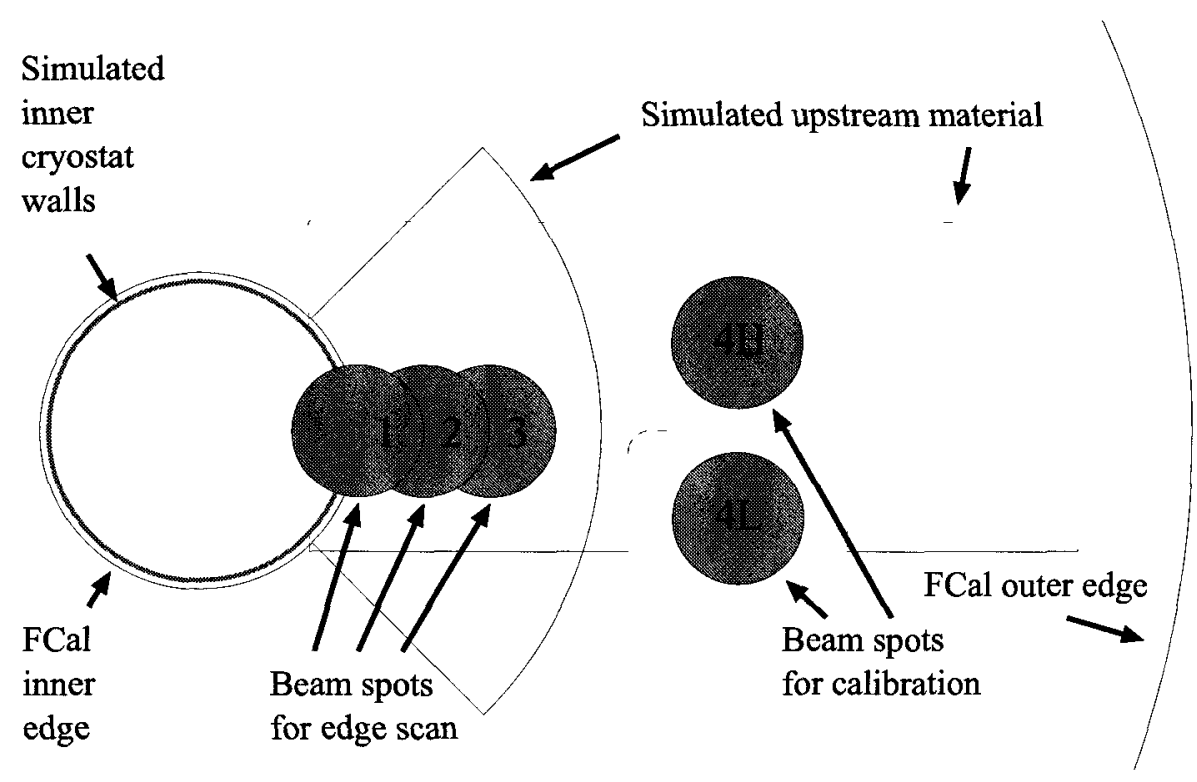

Figure 3.7: The location of the five beam impact points, labelled 1,2,3, 4H, and $4 \mathrm{~L}$, superimposed on the front face of FCal1. The inner and outer edge of the FCal can be seen.

For the test beam, particle beams of electrons and hadrons with a range of energies ( $10 \mathrm{GeV}$ to $200 \mathrm{GeV}$ ) were directed into five locations on the front face of FCal1; these five positions are shown in Figure 3.7. A wide beam profile with a width in $x$ and $y$ of $6.5 \mathrm{~cm}$ was chosen to ensure uniform sampling across the front face and reduce the impact point dependence of the quoted results [18]. The beam impact points are labelled position 1, 2, 3, 4H (high), and 4L (low). Positions 1, 2, and 3 were used to study the energy loss and showering close to the beam pipe. Positions $4 \mathrm{H}$ and $4 \mathrm{~L}$ were located in a region of the detector that is expected to fully contain electromagnetic and hadronic showers up to the maximum test beam energies of $200 \mathrm{GeV}$. Position $4 \mathrm{~L}$ had a negligible amount of upstream material (only the essential upstream triggering and monitoring detectors). The latter was used to investigate the intrinsic response of the calorimeter. Position $1,2,3$, and $4 \mathrm{H}$ had additional upstream material to simulate the expected amount of upstream material in ATLAS, such as the beam pipe and associated services, and the cryostat structure. An aluminum plate $5.0 \mathrm{~cm}$ 


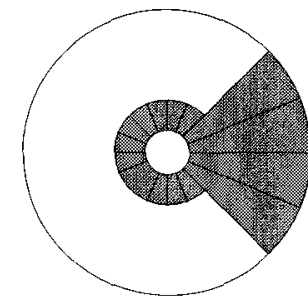

FCal1C

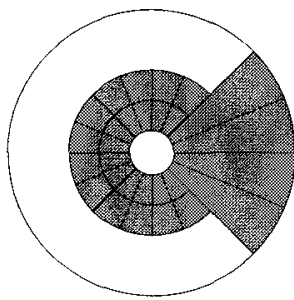

FCal2C

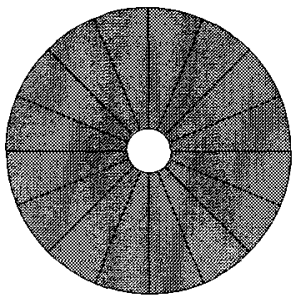

FCal3C

Figure 3.8: The shaded regions are the calorimeter regions of each FCal module that were read out during the test beam.

thick was used for all but position $4 \mathrm{~L}$ to simulate the material from the cryostat bulkhead. In front of positions 1, 2, and 3 a $10 \mathrm{~cm}$ thick polyethylene block was added to simulate shielding that exists in the ATLAS environment. At position 1, a $30 \mathrm{~mm}$ thick aluminum block was added to simulate a vacuum pump used in ATLAS, approximately $900 \mathrm{~mm}$ upstream of the FCal. The shapes and location of this upstream material can be seen in Figure 3.7.

In the test beam the FCal readout was not fully instrumented. The location of the channels that were read out in each module are represented by the shaded regions in Figure 3.8. For the test beam these areas were sufficient to collect the energy deposited by the particle showers. In FCal1, FCal2, and FCal3 there were 432, 308, and 252 channels read out, respectively, for a total of 992 active channels.

The particle types and energies used at each position are shown in Table 3.3. The electron data were composed of mixed polarity runs. The hadron beams were a mixture of positive hadron beams $\left(\pi^{+}\right.$, protons), and relatively pure $\pi^{-}$beams. The choice of polarity was largely influenced by the requirements of other test beam facilities at the same time.

The test beam was conducted at CERN, using the beam from the SPS to generate the particles. Beams from the SPS were directed into an experimental test beam facility called $\mathrm{H} 6$. The $\mathrm{H} 6$ beam line has the ability to generate secondary electron 
or hadron beams (or tertiary for lower energy electrons) with the required range of energies. The last bending magnet seen by the beam provided the vertical deflection for the three different $y$-coordinate impact points on the FCall front face.

\subsubsection{Beam Line Detectors and Associated Data Quality Cuts}

The particle beams encountered many different detectors both upstream and downstream of the FCal. These were used for particle identification, triggering, and tracking. A schematic representation of the beam line is shown in Figure 3.9. The FCal modules were situated in a cryostat filled with liquid argon. The cryostat was rotated and translated as needed for the five different impact points along the $x$ coordinate to align it to simulate beam particles originating at the ATLAS IP.

The proton beam from the SPS would strike a target, then the required particle type and energy would be directed to the $\mathrm{H} 6$ beam line using a series of magnets. If the requested beam particles at the $\mathrm{H} 6$ beam line were $100 \mathrm{GeV}$ electrons, the final beam could contain a mixture of other negatively charged particles, such as muons and $\pi^{-}$. This contamination must be reduced or removed from the analysis to get the most accurate description of the detector response to electrons and hadrons, separately.

\begin{tabular}{|c|r|r|rr|}
\hline \hline & & \multicolumn{3}{|c|}{ Nominal Beam Energy [GeV] } \\
Position & $|\eta|$ & 200 & & Hadrons \\
\hline 1 & 4.9 & 200 & & 200 \\
2 & 4.5 & 200 & & 200 \\
3 & 4.3 & Electrons & 200 \\
$4 \mathrm{H}$ & 3.7 & $10,20,40,60,80,100,150,200$ & $10,20,40,60,80,100,120,150,200$ \\
$4 \mathrm{~L}$ & 3.7 & $10,20,40,60,80,100,150,200$ & $10,40,60,80,100,120,150,200$ \\
\hline
\end{tabular}

Table 3.3: The test beam impact points, and associated beam particle types and energies. 


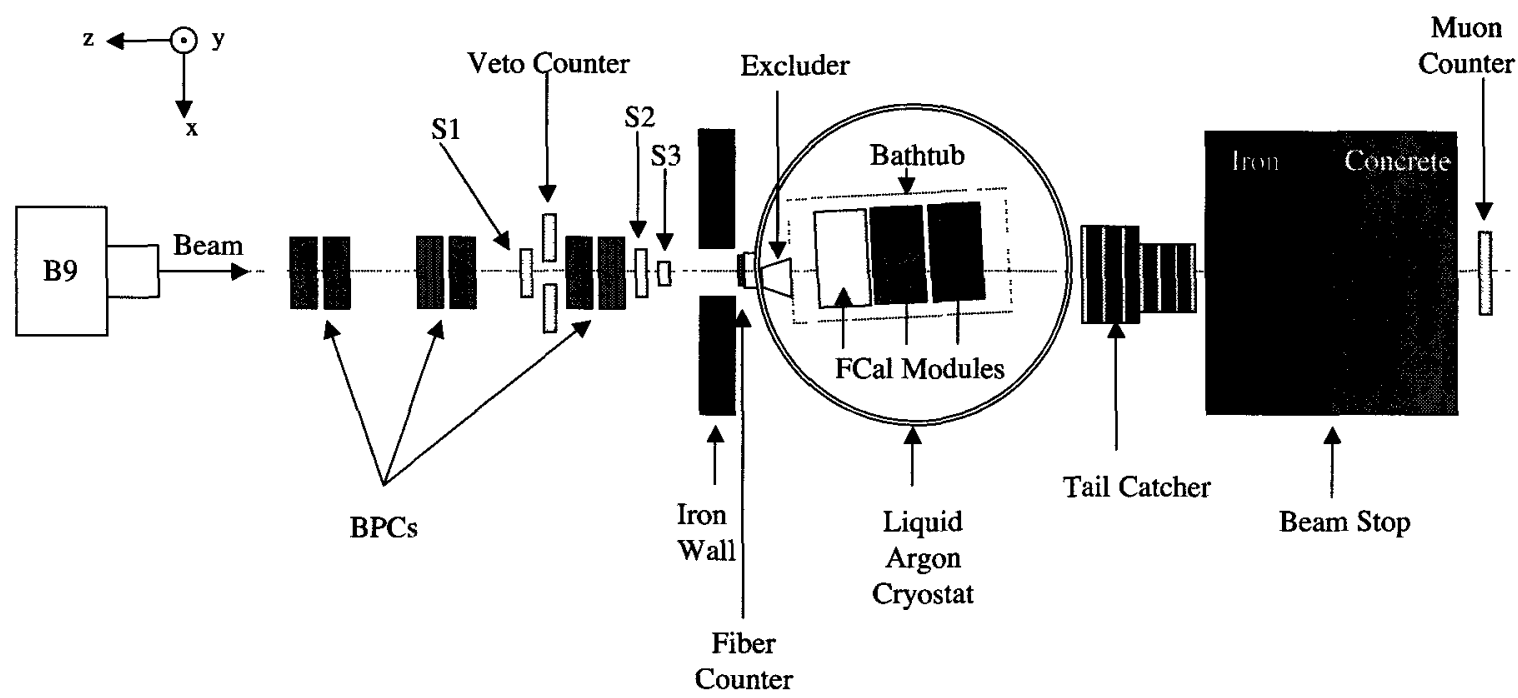

Figure 3.9: Experimental beam line setup. Not to scale. Image from [19].

It is possible that on route to the FCal, along the beam line and associated elements, single particles may undergo interactions. Upstream interactions might alter the trajectory of the incoming particle and cause it to impact the calorimeter outside the required beam spot location, possibly resulting in incomplete shower containment.

Multiple particles from upstream interactions or halo particles associated with the beam line could enter the calorimeter, instead of a single particle. This can lead to false signals in the calorimeter, and may lead to false high energy tails in the response.

The process of removing contamination, multiple interactions, and scattered particles is called beam cleaning and was achieved by examining the properties of the beam line detectors. The following describes the beam line detectors and the associated beam cleaning cuts that were applied to the data.

\section{Scintillators}

Upstream of the FCal were several scintillators. In Figure 3.9 the scintillators labelled $\mathrm{S} 1, \mathrm{~S} 2$, and $\mathrm{S} 3$ were used for coincidence triggering. The scintillator S1 was the first 

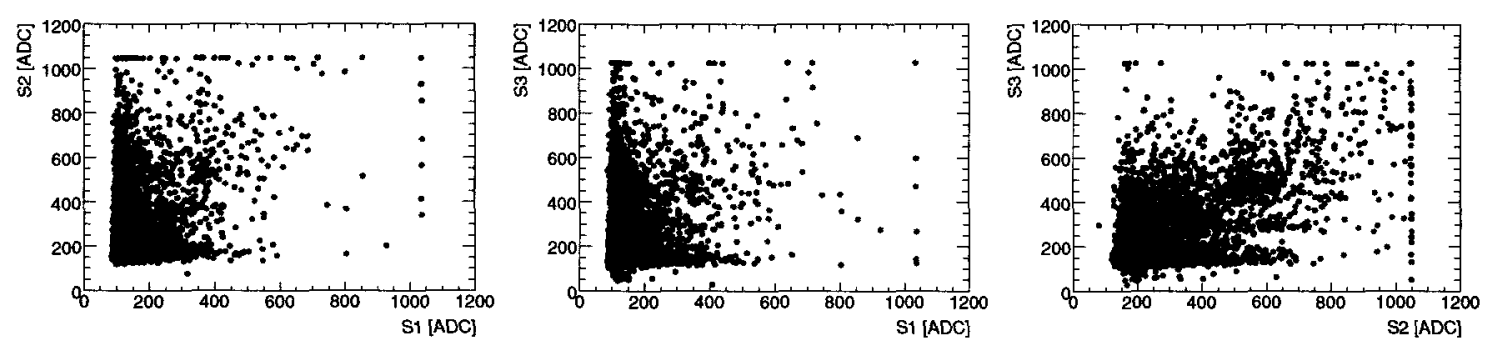

Figure 3.10: An example of the response of the three upstream scintillators S1, S2, S3 in ADC counts. Shown in the black circles are all events, shown in the lighter shade are those events which are rejected, as described in the text.

traversed by the beam and was used as a trigger for the start of the electronics readout. Each of these three scintillators were $1.0 \mathrm{~cm}$ thick.

In the analysis the scintillators $\mathrm{S} 1, \mathrm{~S} 2$, and $\mathrm{S} 3$ were required to trigger in coincidence, indicating a particle coming down the beam line, removing random particle interactions from outside sources.

The response of the three scintillators was used to reject events with multiple particles. The mean response of the scintillators to single minimum ionizing particles (muons) was determined using events in which the downstream muon trigger was fired (indicating the presence of a muon particle). Events were cut in which more than one scintillator had a response compatible with at least two minimum ionizing particles, or events in which the response in one scintillator had a signal consistent with five minimum ionizing particles [20]. Figure 3.10 show an example of the scintillator responses and the data which were cut from the analysis due to large signals in the individual, or combined, scintillators.

\section{Beam Position Chambers}

Three beam position chamber (BPC) stations, upstream of the FCal , provided a total of six measurements of both the $x$ and $y$ coordinates of the incoming particle trajectory (two at each station). The first set of BPCs were approximately $31 \mathrm{~m}$ 
upstream of the FCal, the second set about $22 \mathrm{~m}$, and the last set were approximately $3 \mathrm{~m}$ upstream of the FCal. These were used in the offline analysis for event-by-event track reconstruction and beam quality studies. The approximate location of each set of BPCs along the beam path are indicated on Figure 3.9. The position resolution of the first and last BPCs was approximately $200 \mu \mathrm{m}$, and the resolution of the middle station was $300 \mu \mathrm{m}$.

A straight line was fit to each of the six $x$ and $y$ BPC coordinates. If the sum of the $\chi^{2}$ for the $x$ and $y$ linear fit was less than 40 (with $12-2$ degrees of freedom) the event was accepted for analysis. An analysis was also performed to determine the average response of each $\mathrm{BPC}$ to minimum ionizing particles; if more than three of the 12 BPCs had a signal consistent with two tracks or more the event was cut, or if a single BPC had a response compatible with more than five tracks, the event was cut $[17]$.

\section{Veto Counter}

The veto counter was a scintillator with a circular cutout of $6.5 \mathrm{~cm}$ in diameter, used to remove events with particles that may have scattered outside the window of the beam spot. If this scintillator was triggered the event was removed from the analysis.

\section{Tail Catcher and Muon Scintillator}

Downstream of the FCal was a tail catcher made of alternating steel and scintillator layers. This additional detector allowed for energy measurement of any leakage out the back of the FCal. After the beam stop there was a scintillator used to identify muons.

The two downstream detectors were used for removing hadrons and muons from the electron beams. They were not used for the hadron analysis, as there can be legitimate decays of hadrons into muons. They can be used to calculate the amount 
of energy leaked from the back of the calorimeter or the inner radius of the FCal.

\section{CEDAR Trigger}

Not shown on Figure 3.9 is a Čerenkov differential counter with achromatic ring focus (CEDAR) positioned upstream of the magnet labelled B9 on the figure. It provided particle identification of the incoming particle.

The CEDAR was calibrated to remove protons from the positive polarity hadron beams at all energies except $200 \mathrm{GeV}$. The $60 \mathrm{GeV}$ electron beam also suffered large hadron contamination, and so the CEDAR was tuned to remove hadrons from this electron energy point only.

In addition to the above cuts, if any beam line detector actively used in the analysis was unavailable, the run or subset of events in that run removed from the analysis.

\subsubsection{Event Timing}

On the front end boards, signals from the FCal electrodes were sampled every $25 \mathrm{~ns}$. This time was recorded with the $40 \mathrm{MHz}$ trigger timing control (TTC) clock. The beam triggers arrived asynchronously with respect to the TTC clock. Exact signal timing with respect to the incoming particle was essential for signal reconstruction (as will be discussed in Section 3.6). So the relative time phase $t_{\text {phase }}$ between the beam trigger (from S1) and the TTC clock was calculated for each event as:

$$
t_{\text {phase }}=\alpha\left(t_{\mathrm{tdc}}-k\right) \quad[\mathrm{ns}]
$$

where $\alpha$ is the time-to-digital converter (TDC) calibration factor (ns/bit), an intrinsic

property of the TTC clock. The variable $t_{\mathrm{tdc}}$ is the TDC value for a given event, 


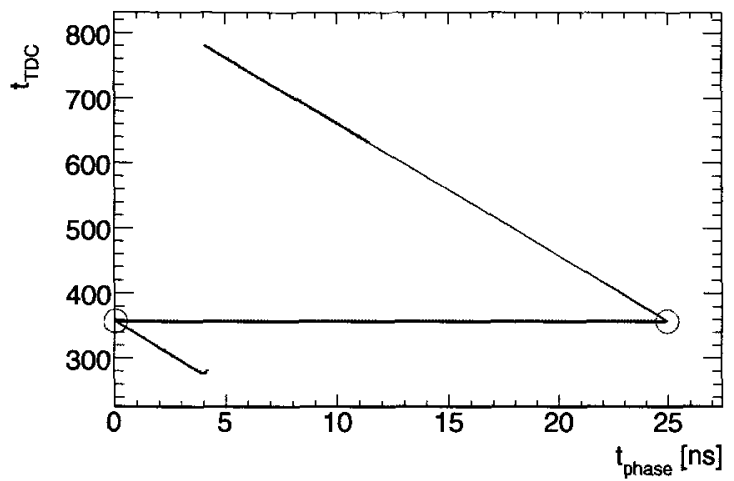

Figure 3.11: Example of a subset of the test beam data showing the TDC versus phase, used to calculate the wrap around constant. The horizontal line represents the location of the wrap around constant, taken as the average of the two extremum points in each circle.

starting from the S1 trigger and ending with the TTC clock sampling, which has a precision of 50 ps. The constant $k$ is known as the wrap around constant (WAC) in TDC counts, which signifies the difference in phase between the beam clock and the TTC clock. Figure 3.11 shows an example using a subset of the test beam data of the TDC value versus phase. The location of the split in TDC counts (approximately $360)$ is the wrap around region in this case. To find the WAC the average is taken of the two points indicated by circles.

Events that were too close to the WAC region may be ambiguous and shift the time by $25 \mathrm{~ns}$; such events were cut from the analysis. To increase statistics and maintain good event timing information a second TDC input was used, offset to the first by approximately $10 \mathrm{~ns}$. The clock which had its TDC reading farthest from its maximum, minimum, and WAC region was used for the event timing in the analysis. However even in the rare cases that the best clock choice was within 1 ns of its maximum, minimum, or WAC regions that event was excluded from the event. The stability of the WAC for both TDC counters as a function of time (run number) are shown in Figure 3.12. 


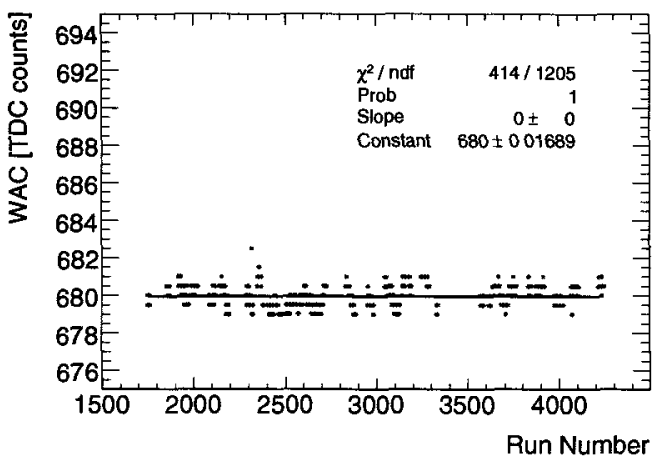

(a) Wrap around constant stability for one of the TDC clocks.

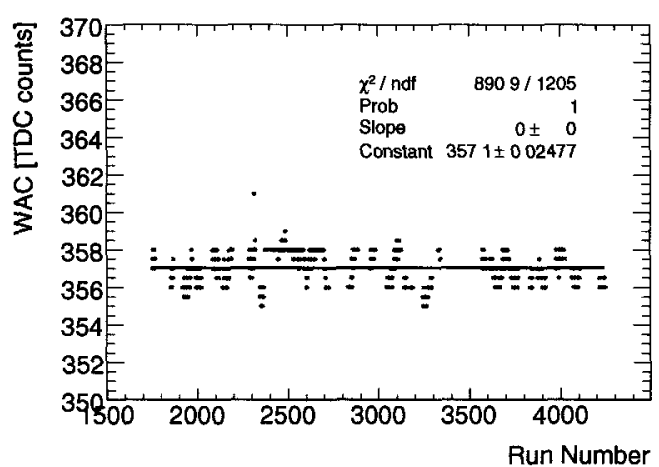

(b) Wrap around constant stability for the second TDC clock.

Figure 3.12: Wrap around constant stability over the full test beam data taking period for both TDC counters.

\subsubsection{Summary of Runs Taken}

The physics data taken had approximately $200 \mathrm{~K}$ triggers for electrons at each energy and position. For hadrons there were approximately $400 \mathrm{~K}$ triggers for each energies for position $4 \mathrm{H}$ and $4 \mathrm{~L}$, and $600 \mathrm{~K}$ for the single energy beams in positions 1,2 , and 3 . The fraction of events remaining after offline selection cuts varies with particle type and energy (a reflection of the beam quality).

There were several types of data recorded during the test beam:

Physics Runs: Using real beam particles the signal was sampled seven times every 25 ns primarily in autogain mode (for all but the highest energy electrons this meant high gain). More details on the signal sampling are discussed in the next section. Each run had approximately 12000 beam triggers.

Random Data: Within the physics runs $5 \%$ of the triggers were events triggered at random, not corresponding to the incoming particle. These were taken to calibrate the baseline readings for the readout channels.

Calibration Runs: When there was no beam present, a pulse from the calibration board was sent through the electronics to study the response of the front end 
boards and cold electronics.

The following sections describes how the signals from each channel were reconstructed.

\subsection{Reconstruction of the Electronic Signal}

The current pulse collected on the anode and read out through the electronics stems from the ionization of the liquid argon and drifting of the ionized electrons across the gap. In the FCal, the pulse was sampled every $25 \mathrm{~ns}$, with the intent of reconstructing the bipolar pulse shape. The maximum height of the pulse in a readout channel is proportional to the number of ion pairs produced, which is a measure of the energy deposited by the incoming particle in that channel. The conversion from current to ADCs is calculated channel-by-channel by inputting an electronics signal of known current and seeing the ADC response. The average response is taken as the conversion constant between the input current and ADC counts, when summing over all channels.

The FCal has a pulse shape that rises quickly in time and then falls off linearly as the electrons drift across the gap. When put through the electronics chain the resulting physics pulse shape read out for each FCal module takes the form shown in Figure 3.13. For one module the pulse shape is identical for all channels, as each channel has the same liquid argon gap size and the same shaper is applied to the pulse. The slight differences in pulse shapes between modules reflect the different gap sizes and therefore the different electron drift times.

In the test beam setup the pulse shape was sampled seven times every $25 \mathrm{~ns}$ ( 7 $\times 25 \mathrm{~ns}=175 \mathrm{~ns})$. For ATLAS data taking the nominal pulse sample rate is five times every $25 \mathrm{~ns}$. Sampling the pulse yields discrete points on the pulse, such as those shown in Figure 3.14. The maximum pulse height can be estimated by three 

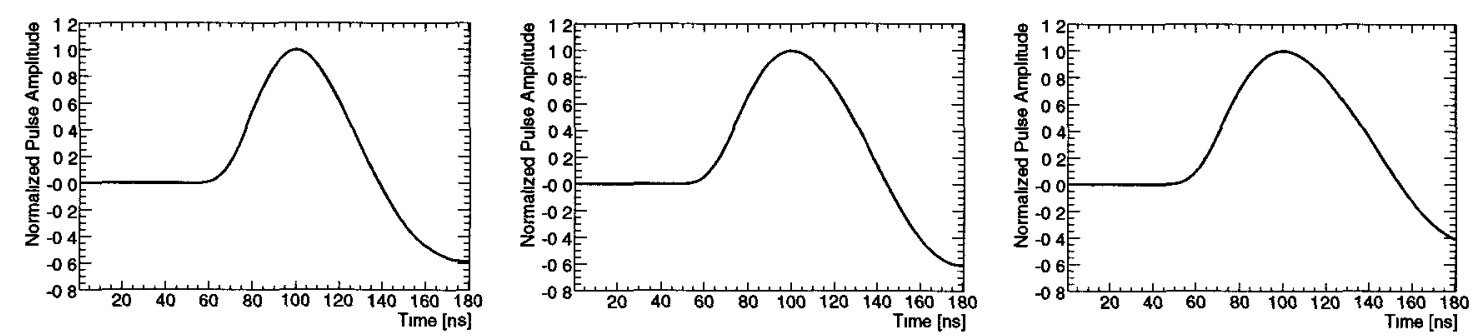

Figure 3.13: The normalized pulse shape for FCal1 (left), FCal2 (middle), and FCal3 (right), taken from SPICE simulations [21] of the FCal electronics readout [22] (and later verified using test beam data [23]).

methods:

Maximum Sample: Take the maximum sample as the maximum height of the pulse.

Parabola Fit: Fit the largest samples (sample two to five) with a parabola and find the peak of the parabola fit.

Optimal Filtering Technique: A method which reconstructs the peak amplitude using the samples and information about the true pulse shape and characteristics of the electronics [24].

Figure 3.14 demonstrates these three techniques, with the parabola fit shown with a solid line and the Optimal Filtering (OF) fit with the dashed line. Both fits are better at identifying the maximum peak than taking the maximum sample value. The OF reconstruction fits all the sample points well, whereas the parabola fits only the central points. The method that best reproduces the true pulse, and hence the true maximum is the OF technique, as it contains the most information about the true pulse characteristics, including timing, and individual channel traits. 


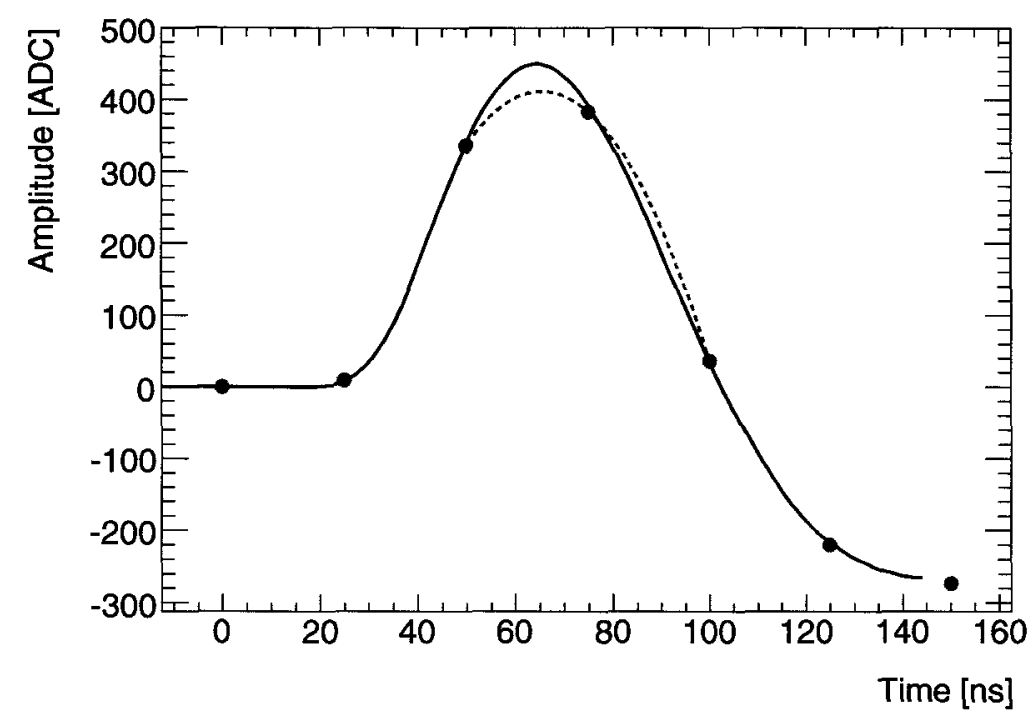

Figure 3.14: Example of the seven samples (filled dots) of the pulse shape taken from a channel in FCall with a physics signal from the test beam. Superimposed is a fit to a parabola (solid line), and the fit from the optimal filtering technique (dashed line).

\subsubsection{Optimal Filtering Technique}

The OF technique makes use of information about the pulse shape and variations of the electronics readout (noise, which is described further in Section 3.6.2) in a particular channel. The authors of this method describe the method in the following way:

"In the context of calorimeter signal processing, optimal fltering refers to the formation of linear combinations of signal samples to recover the signal parameters, namely the amplitude $A$ and start time $\tau$, while minimizing the effect of noise." [24]

Consider that the normalized physics pulse shape in one channel is given by $g(t)$, with the peak centered on some reference value (given by the true pulse shape), such as those shown in Figure 3.13. In reality the time of this peak can vary from channelto-channel with respect to this reference pulse by some small amount $\tau$ due to small differences in the time taken for the signal to be read out (particle travel time, cable 
lengths, etc.). The pulse will have some amplitude $A$, proportional to the energy of the incoming particle. The sample $S$ at time $t_{\imath}$ can be described by:

$$
S\left(t_{\imath}\right)=A g\left(t_{\imath}-\tau\right)+N_{\imath},
$$

where $N_{\imath}$ is the additional noise introduced by electronics that causes deviations from the ideal pulse shape. For small $\tau$ this can be expanded in a Taylor series to become:

$$
S\left(t_{\imath}\right)=A g\left(t_{\imath}\right)-A \tau g^{\prime}\left(t_{\imath}\right)+N_{\imath},
$$

where $g^{\prime}\left(t_{\imath}\right)=d g(t) /\left.d t\right|_{t=t_{2}}$.

The above is a description of the pulse sampled at a discrete time $t_{\imath}$. In the test beam the amplitude and time reconstruction is based on seven discrete samplings of the pulse. In general, for $n$ samples, optimal filtering coefficients $a\left(t_{\imath}\right)$ and $b\left(t_{\imath}\right)$ are defined in the following equations:

$$
\begin{aligned}
A & =\sum_{\imath=1}^{n} a\left(t_{\imath}\right) S\left(t_{\imath}\right)=\sum_{\imath=1}^{n}\left[A a\left(t_{\imath}\right) g\left(t_{\imath}\right)-A a\left(t_{\imath}\right) \tau g^{\prime}\left(t_{\imath}\right)+\left\langle N_{\imath}\right\rangle\right] \\
A \tau & =\sum_{\imath=1}^{n} b\left(t_{\imath}\right) S\left(t_{\imath}\right)=\sum_{\imath=1}^{n}\left[A b\left(t_{\imath}\right) g\left(t_{\imath}\right)-A b\left(t_{\imath}\right) \tau g^{\prime}\left(t_{\imath}\right)+\left\langle N_{\imath}\right\rangle\right]
\end{aligned}
$$

The noise is a random fluctuation about zero, hence it will average to zero after summing over all $n$ samples, in this approximation $\left\langle N_{\imath}\right\rangle=0$. To satisfy Equations 3.16 and 3.17 requires that:

$$
\begin{aligned}
& \sum_{\imath=1}^{n} a\left(t_{\imath}\right) g\left(t_{\imath}\right)=1 ; \quad \sum_{\imath=1}^{n} a\left(t_{\imath}\right) g^{\prime}\left(t_{\imath}\right)=0 \\
& \sum_{\imath=1}^{n} b\left(t_{\imath}\right) g\left(t_{\imath}\right)=0 ; \quad \sum_{\imath=1}^{n} b\left(t_{\imath}\right) g^{\prime}\left(t_{\imath}\right)=-1
\end{aligned}
$$


A further condition on the OF technique is to minimize the variance of the amplitude and timing resolution:

$$
\begin{aligned}
\sigma^{2}(A) & =\sum_{\imath=1}^{n} \sum_{\jmath=1}^{n} a\left(t_{\imath}\right) a\left(t_{\jmath}\right) R_{\imath \jmath} \\
\sigma^{2}(A \tau) & =\sum_{\imath=1}^{n} \sum_{\jmath=1}^{n} b\left(t_{\imath}\right) b\left(t_{\jmath}\right) R_{\imath \jmath}
\end{aligned}
$$

where $R_{\imath \jmath}$ is the noise autocorrelation matrix between samples $\imath$ and $j,\left\langle N_{\imath} N_{\jmath}\right\rangle$ of a channel. The noise autocorrelation between samples is obtained from calibration data for each channel between the $n$ samples. These constraints lead to the extraction of the OF coefficients.

In the test beam data these coefficients were extracted using the FCal pulse shapes in Figure 3.13 to define $g\left(t_{\imath}\right)$, and from selected channels within physics runs. The channels chosen were those with significant energy deposition over the electronic noise ( $A>5 \times \sigma)$, to ensure the resulting pulse shape was that of a physics pulse shape, and not noise. More details of this procedure are found in [23].

\subsubsection{Pedestal and Noise}

The pedestal is the mean baseline value of a readout channel in the absence of a physical energy deposition, and the variation of this pedestal is called the electronic noise. It is a consequence of the electronics chain. In this analysis the pedestal for one channel was calculated by using the first sample (of seven) read out for physics triggered events, averaged over all data for one run. The distribution of the pedestals follows a Gaussian distribution, and the one standard deviation spread of the pedestals is defined as the noise. Figure 3.15 shows an example of the first sample for all physics events in one run for one channel. 


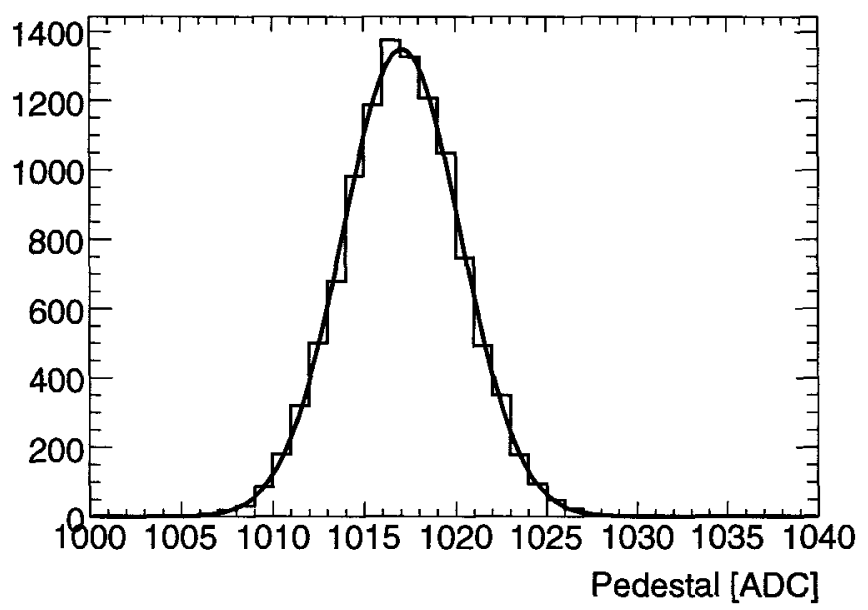

Figure 3.15: Example distribution of the first sample for one channel in one run. A Gaussian fit is superimposed. For this channel and run the pedestal is 1016.6 ADC counts (mean of the distribution) and the noise is $3.2 \mathrm{ADC}$ counts (one sigma spread of the distribution).

The run range taken for the test beam data spans from run 1749 to 4315 , and excludes runs deemed as bad, due to poor experimental conditions. The average pedestal per channel over all good runs is shown in Figure 3.16(a). The pedestal was approximately constant across all 992 active FCal channels. Figure 3.16(b) shows the stability of the pedestal averaged over all channels for the good runs used in analysis. Gaps along the $x$-axes in Figure 3.16(a) are a consequence of inactive channels, and in (b) for runs with poor experimental conditions. When the channel signal is reconstructed the pedestal for a given channel in a specific run is subtracted from the individual samples. In this way it is possible for a sample to have negative 'energy', due to fluctuations of the pedestal.

Alternative methods of calculating the pedestal and noise are by using the first sample from random triggered events, or by applying the full OF reconstruction to extract the amplitude from randomly triggered events. The latter is used for the noisesubtracted energy resolution analysis (Section 3.8), and is also used in the channel clustering decision (Section 3.7). The three methods of calculating the noise are 


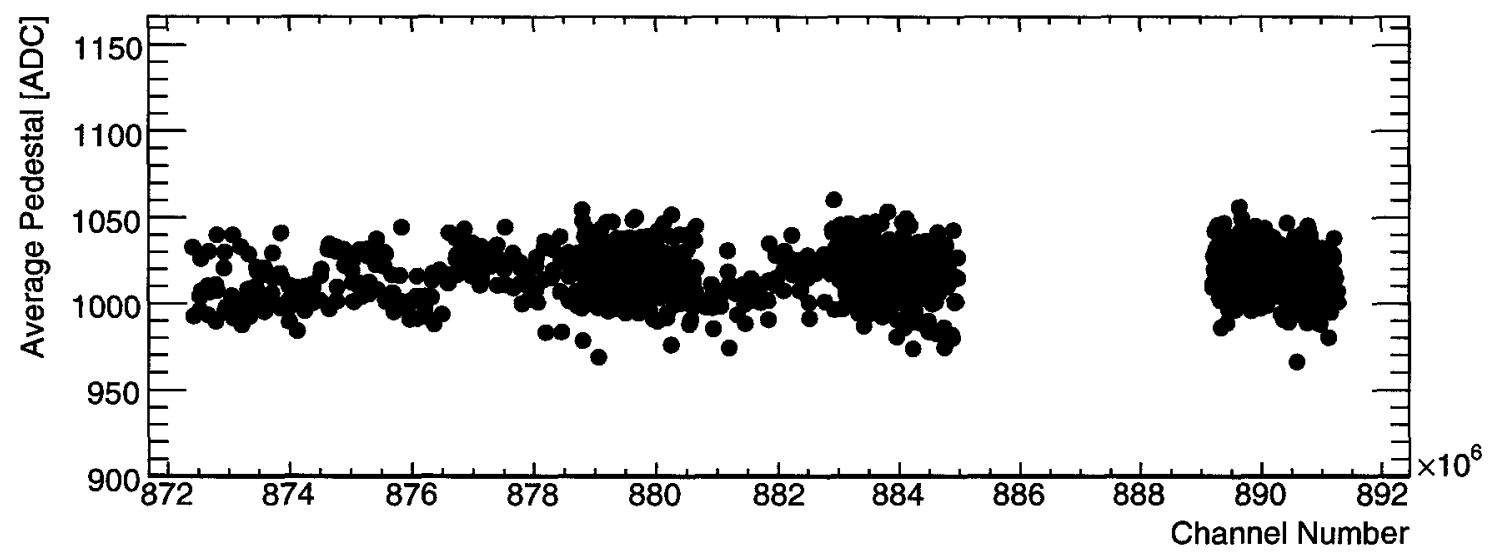

(a) Average pedestal versus channel number, averaged over all data.

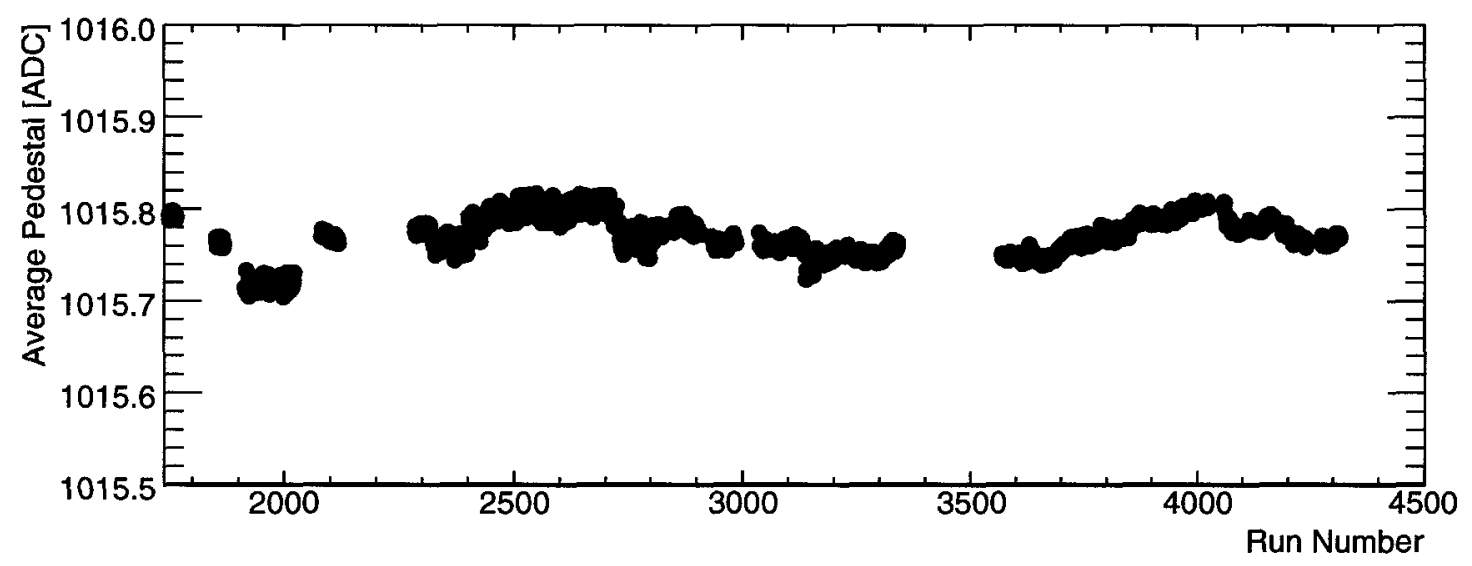

(b) Average pedestal versus run number, averaged over all channels.

Figure 3.16: Average pedestal values for all data and channels used in this analysis. 
shown in Figure 3.17 for the average (over all channels) noise reconstructed (in ADC counts) versus run number in the test beam analysis. In this figure the data points from the first sample from the physics data are equivalent to the data points of the first sample from random triggered events. So the average first sample from physics data yields equivalent noise as the random triggered data in the same run.

When the random triggered data were reconstructed using the OF technique (as was done with the physics data) the average noise is about $70 \%$ of the average noise when the first sample was used. This demonstrates the power of the OF technique in minimizing noise contributions in the reconstruction.

There were a subset of runs that had higher noise contributions due to the setup of the test beam during data taking, this problem was identified and corrected in the remainder of the runs. These runs can be seen in Figure 3.17. These limited noisy runs are a motivation for using run-by-run noise information in the following analysis of the data. The choice of channel-by-channel noise analysis was taken due to the variation in noise observed between channels. This is seen by the scatter of points in Figure 3.18, which shows the average noise versus channel number, averaged over all runs, using the first sample from physics data.

\subsubsection{Summary}

Each of the 992 FCal readout channels of the test beam were reconstructed using the OF technique, on pedestal subtracted data. The final numerical value per electronic channel was a count in ADCs, proportional to the amount of energy deposited by the incoming particle in one specific channel. One goal of the test beam analysis was to verify the predicted conversion between ADC counts and energy. This analysis will be presented in Section 3.8.1. 


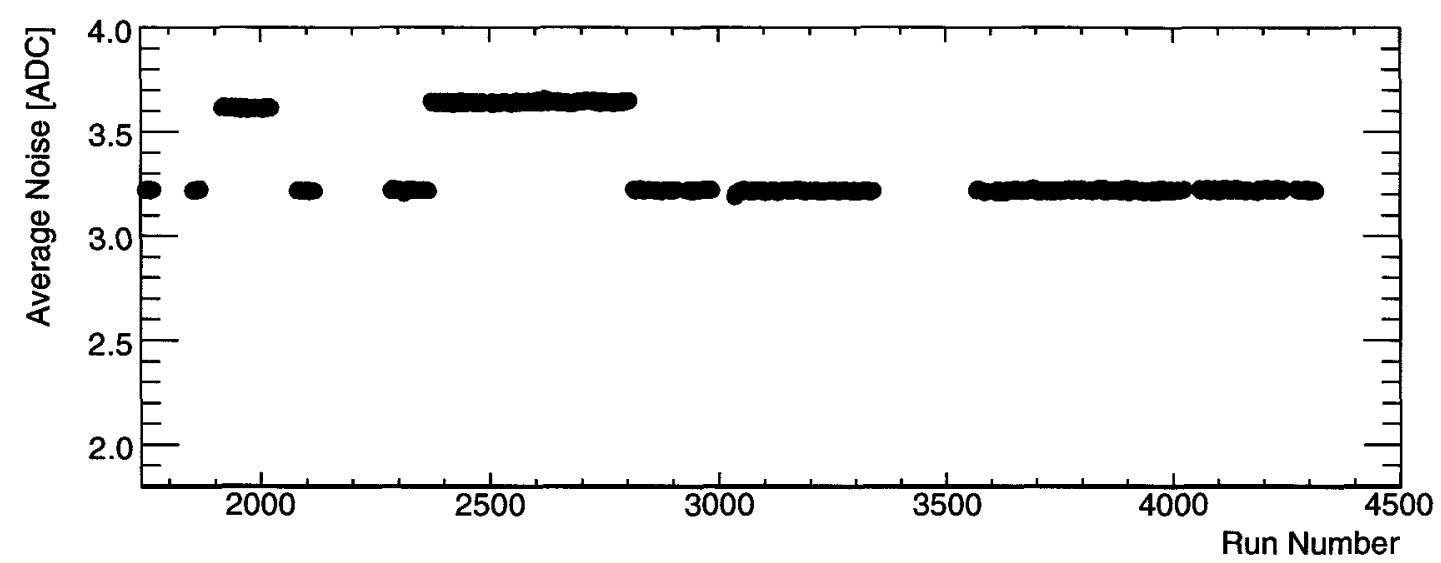

(a) First sample physics data.

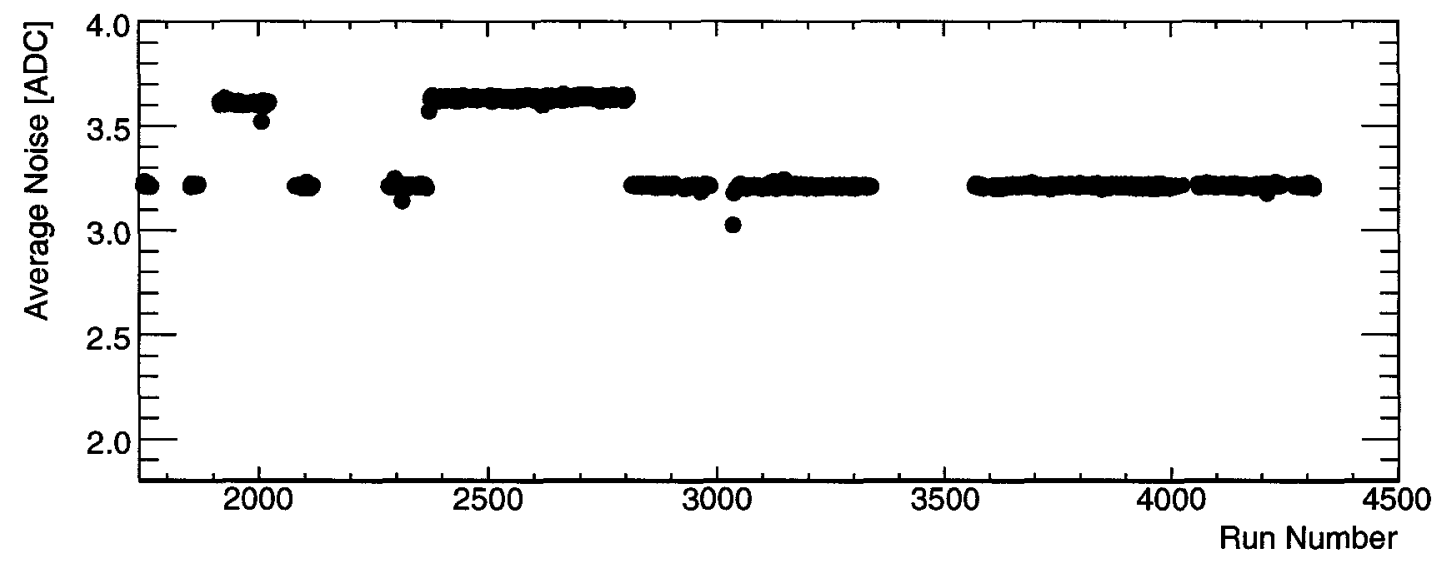

(b) First sample random triggered data.

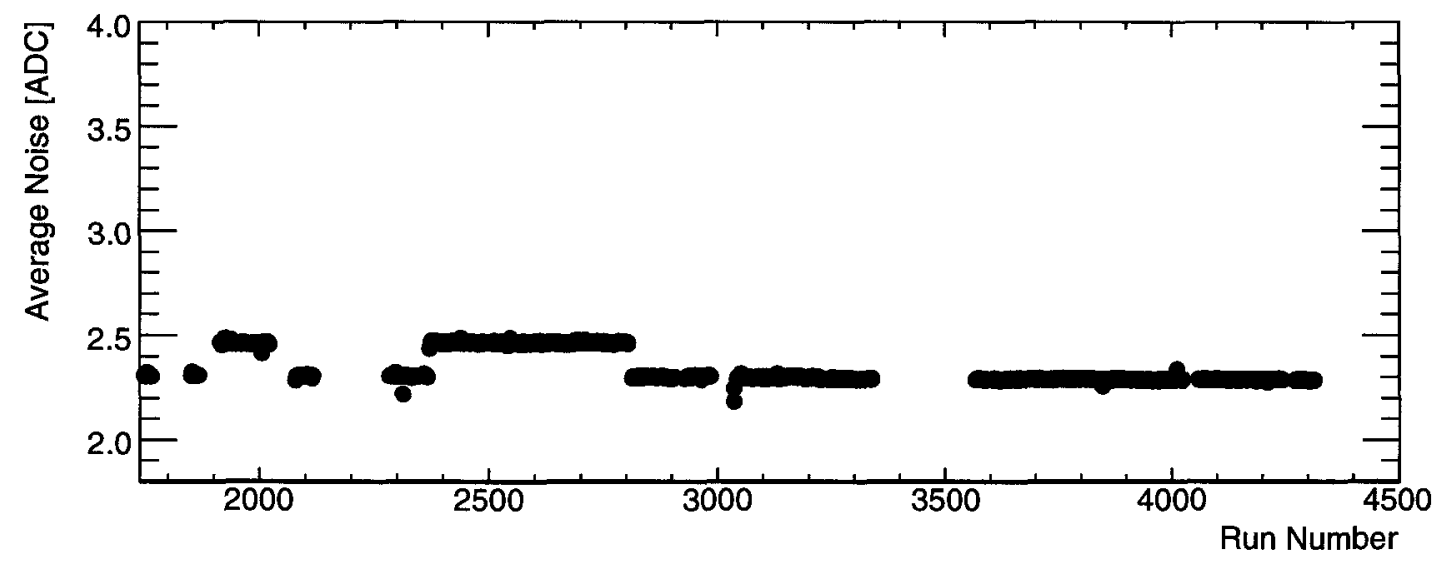

(c) Optimal filtered reconstructed random triggered data.

Figure 3.17: Average noise for all channels as a function of test beam run number. Three methods for calculating the noise are shown: (a) using the first sample (of seven) from physics data, (b) using the first sample from random triggered events, and (c) using the optimal filtering channel reconstruction of random triggered events. 


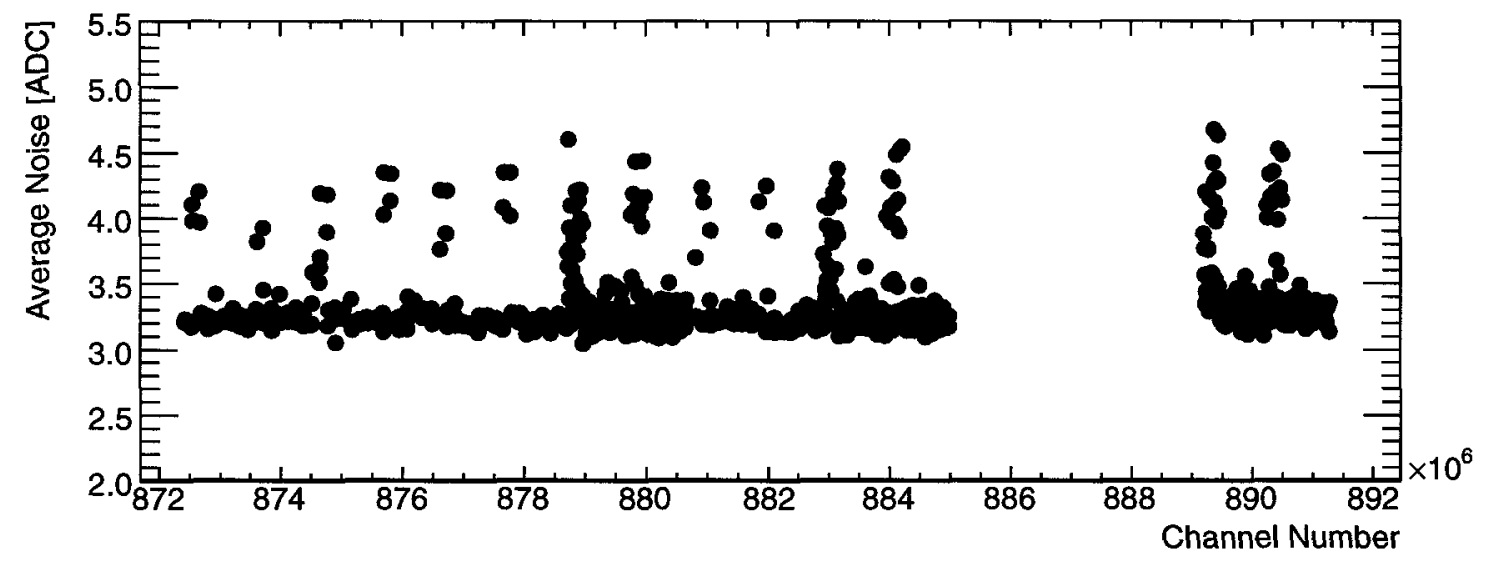

Figure 3.18: Average noise versus channel number averaged over all good runs, where the noise was calculated using the variation in the first sample in physics runs. 


\subsection{Channel Clustering}

Once the signal amplitude and timing has been reconstructed from the raw samples for each channel, it is then necessary to decide whether to sum the contributions from all channels (992 in the test beam) or choose a method that selects a subset of channels to be included in the energy sum. In the case of the test beam it is known that one particle will enter the FCal and deposit its energy in a limited number of channels. The remaining channels will reconstruct noise, which will on average contribute zero to the overall energy sum, but will degrade the width of the energy distribution, and hence worsen the energy resolution. In ATLAS there will be a multitude of particles leaving the IP and hitting the FCal, and it will be necessary to identify those calorimeter channels corresponding to the energy deposition for each outgoing particle. It will also be necessary to associate groupings of channels to different shower types, to apply the appropriate electromagnetic or hadronic shower scale calibrations. The process of selecting which channels will be summed to reconstruct the energy of a particle, or event, is called clustering.

The challenge is to identify those channels with real energy deposition, reflecting the amount of energy of the incoming particle, while minimizing the contribution from noise. In this test beam analysis two methods have been explored: a fixed radial cylindrical clustering algorithm, and a topological clustering algorithm that clusters channels based on their signal to noise ratio.

\subsubsection{Fixed Cylinder Clustering}

In a test beam event there was one particle of known type and energy incident on the front face of FCal1, with a known impact point obtained using information from the upstream BPC track reconstruction. The basic properties of particle interactions with 
matter are well understood and were described in Section 3.1. The Moliére radius of FCal1 is $17.1 \mathrm{~mm}$. For the highest energy electrons in the analysis $(193.1 \mathrm{GeV})$, the electromagnetic showers will have a maximum number of particles in the shower at an average depth of $9.2 X_{o}$ (using Equation 3.6). The depth of FCal1 is $27.6 X_{o}$, hence in almost all cases the electromagnetic showers will be fully contained in FCal1. For these reasons and to be certain all energy of incident electrons are fully collected, the cylindrical clustering algorithm when applied to electrons, will cluster all channels within a fixed radius of $80 \mathrm{~mm}$ from the particle impact position in FCall.

Hadrons have much deeper and wider showers than electrons. For the highest energy hadrons in this analysis, $200 \mathrm{GeV}$, the depth of the shower maximum is (from Equation 3.9) $2.81 \lambda$. FCal1 has a total depth of $2.65 \lambda$ and FCal2 has a total depth of $3.62 \lambda$, so on average the shower maximum will occur in FCal2.

In general the lateral shape of a hadronic shower has a large electromagnetic core out to about the Moliére radius and has a larger hadronic shower that spans a transverse size equivalent to approximately $\lambda$ [25]. Using the known depth of each module in millimeters and radiation lengths it can be calculated that $\lambda$ is $170 \mathrm{~mm}$, $124 \mathrm{~mm}$, and $127 \mathrm{~mm}$, in FCal1, FCal2, and FCal3, respectively. For these reasons in the hadron analysis all channels within a $160 \mathrm{~mm}$ radial distance from the cluster center are summed from FCal1, FCal2, and FCal3.

To perform cylindrical clustering it is necessary to identify the cluster center, and start summing all channels within the fixed cylinder size. Reconstructing a straight line through the BPC hits and projecting along $z$ to each of the modules front faces defines the cluster center. However, the BPCs have their own coordinate system, which has no relationship to the channel $x$ and $y$ positions of the FCal. The last two BPCs closest to the FCal were installed on moveable tables to be able to provide full coverage at each of the five impact positions. The FCal was rotated and translated to 
achieve the changes in $x$ for the five impact positions, requiring a different alignment between the BPC and the FCal coordinate systems for each impact position.

Using the position and energy deposited in the FCal channels, the cluster center (CC) can be calculated using an energy weighted technique, where only channels with a signal to noise ratio of five or greater were used in the cluster center calculation:

$$
\mathrm{CC}_{x}=\frac{\sum_{\imath=1}^{N} E_{\imath} x_{\imath}}{\sum_{\imath=1}^{N} E_{\imath}}
$$

where $i$ is a sum over the channels with the required signal to noise significance $(i=1,2, \ldots, N)$, and $E_{\imath}$ and $x_{\imath}$ are the channel energy and position, respectively. The same formula was applied for the $y$ cluster center coordinate. Data from the highest energy electron and hadron runs were used in this alignment (highest energy test beam data), as these were more likely to have larger lateral energy distributions, increasing the number of channels in the summation, and reducing the bias towards channel centers that forms due to discrete sampling.

A sample distribution of the cluster centers in $x$ and $y$ (for FCal1) are shown in Figures 3.19 for a subset of the electron and hadron data at impact position 4L. There are clear peaks in this distribution that stem from the coarse sampling of the FCal. In FCal1 the distance between channels is approximately $30 \mathrm{~mm}$, on average. When calculating the energy weighted cluster center, the channel with the most energy tends to pull the fit toward its center. So simply using the difference in the projected BPC tracks and the cluster centers for all events would not be correct, due to the bias in the FCal energy-weighted cluster center calculation.

The best events to use for the alignment were those that had a cluster center reconstructed at the mid-point between two channels, in this case it is likely that the energy was equally shared among the neighbouring channels. The actual channel $x$ 

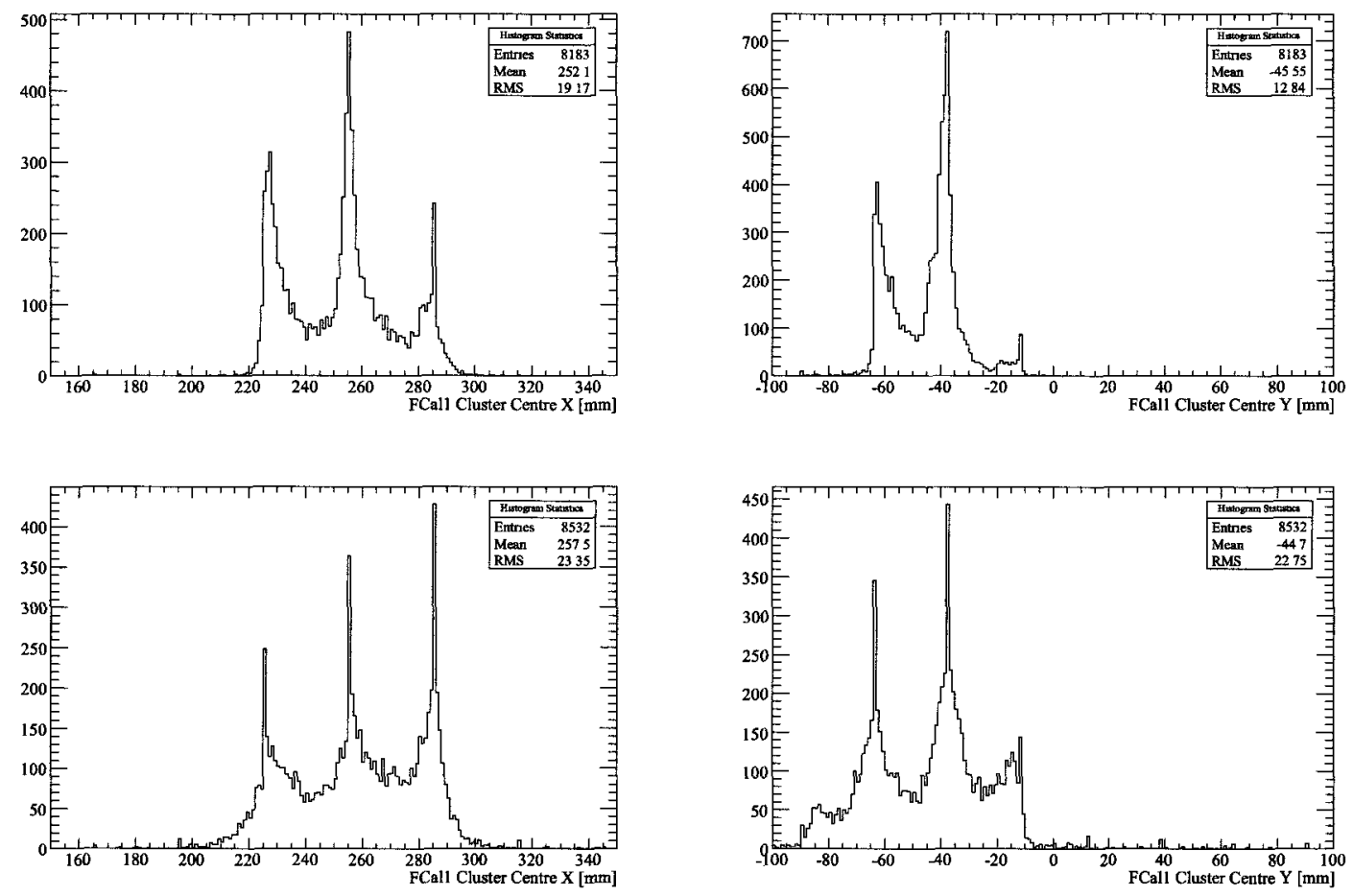

Figure 3.19: The channel energy weighted cluster centers in $x$ (left) and $y$ (right). Shown is a sample run in position $4 \mathrm{~L}$ for electrons (top) and hadrons (bottom). 

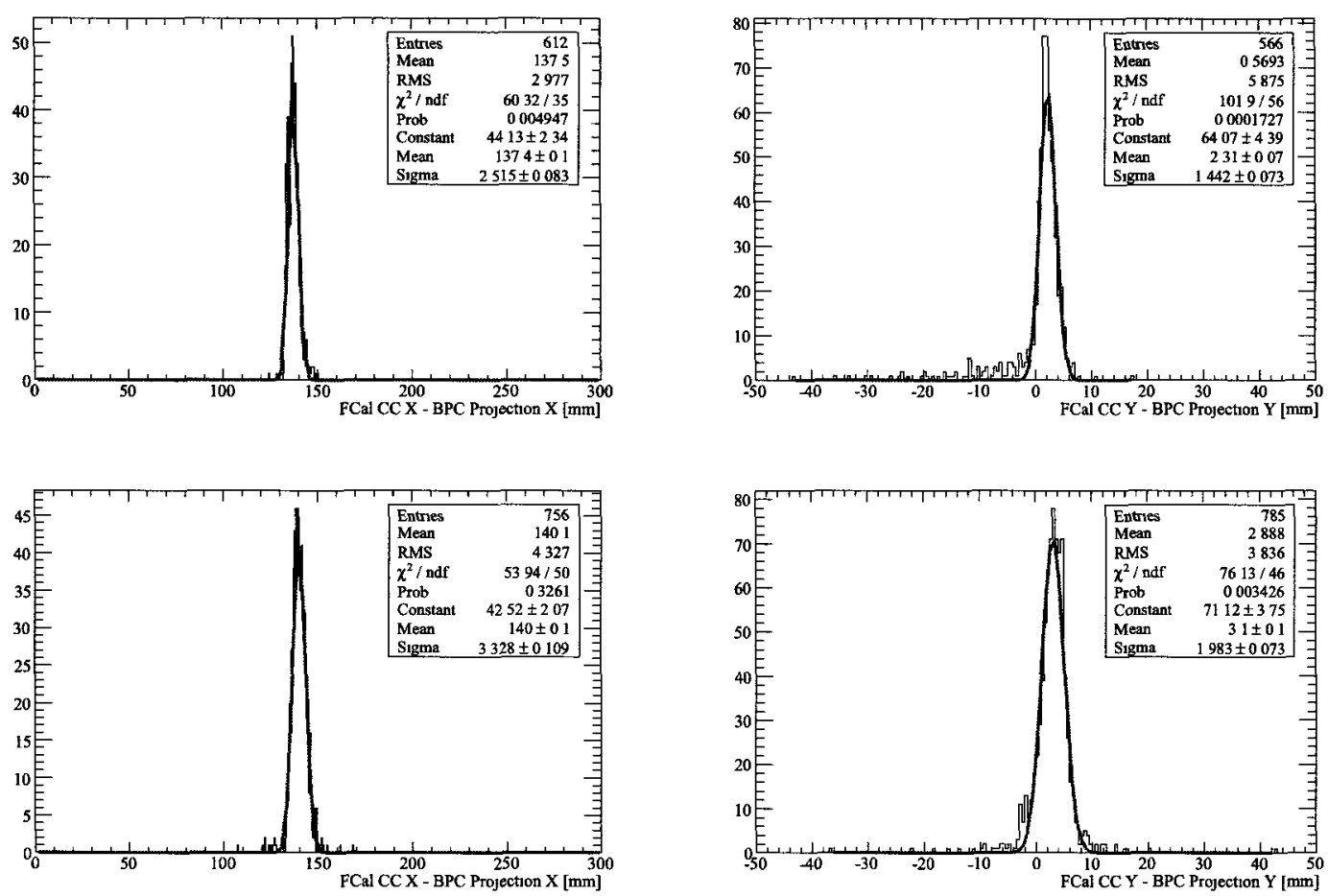

Figure 3.20: Difference between BPC projected track on the FCall front face and the channel energy weighted cluster center. Shown is a sample $200 \mathrm{GeV}$ run at impact position 3 , for electrons (top) and hadrons (bottom).

and $y$ positions are used and the mid-point between neighbouring channels (in $x$ and $y$ ) was calculated. Events that had a reconstructed cluster center in this mid-point trough $( \pm 2.5 \mathrm{~mm})$ were used to align the FCal with the projections from the BPCs. The difference between the projection and reconstructed cluster centers was taken and plotted for both $x$ and $y$. A cut is applied to ensure the linear fit to the six BPCs is good, requiring the $\chi^{2}$ fit probability is better than $0.1 \%$. A sample distribution is shown for $200 \mathrm{GeV}$ electrons in Figure 3.20 (top) and hadrons Figure 3.20 (bottom). Superimposed to the data are Gaussian fits.

The same procedure was applied for each run. The mean of the Gaussian fit was taken as the average alignment shift between the coordinate systems for that particular run. The mean alignment shifts as a function of run are shown in Figure 3.21 

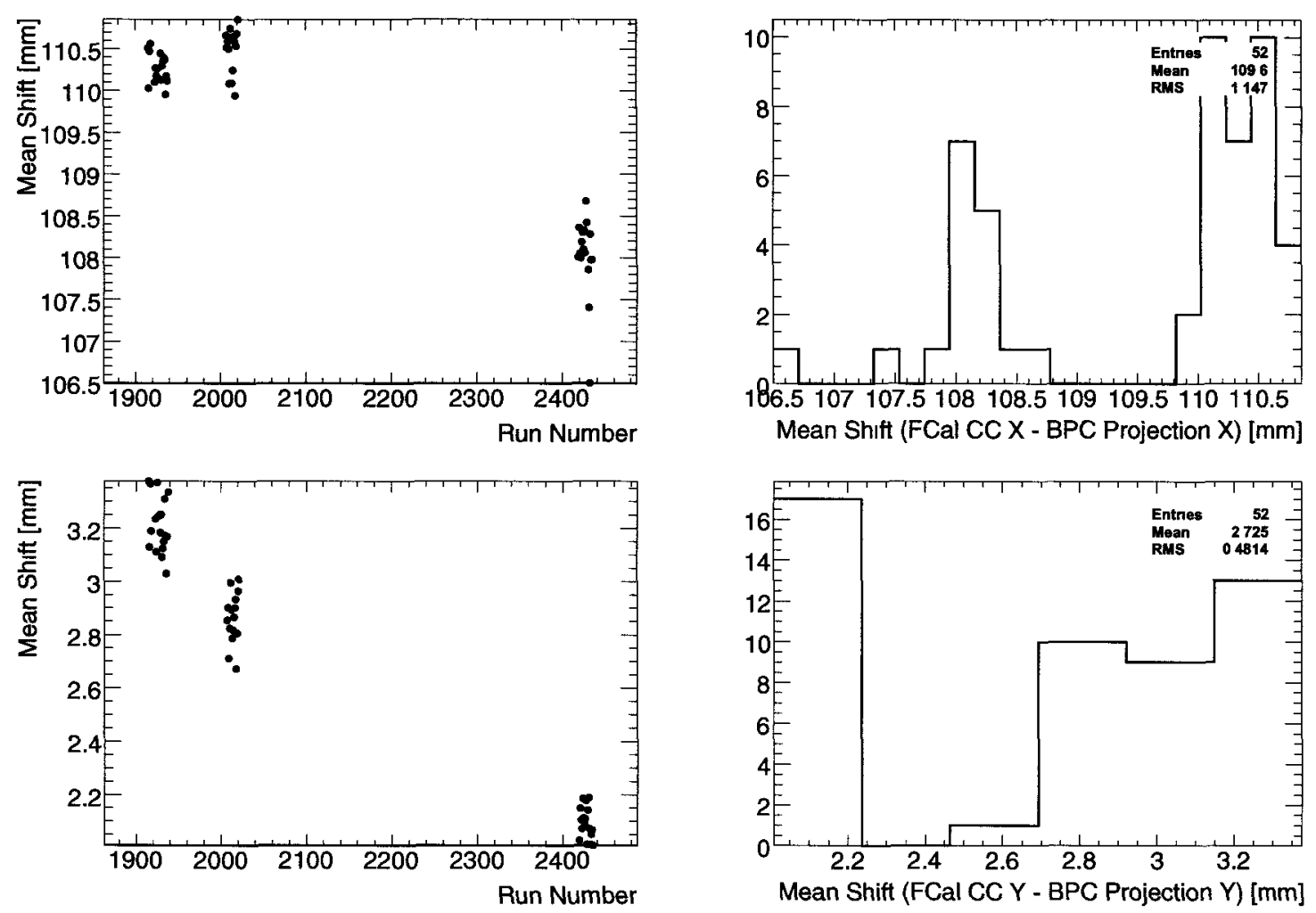

Figure 3.21: The figures on the left show the mean shift versus run number for the $x$ (top) and $y$ (bottom) coordinates at impact position 2. The gaps along the $x$-axis correspond to runs that were not included at impact position 2 . The figures to the right show the distribution of mean shifts for position 2. There are common values for the shift over consecutive run ranges. The differences in the shifts between run periods indicates small differences in the realignment and position of the BPCs and/or FCal cryostat.

for all runs at impact point 2. Similar plots exist for the other four impact positions in the test beam.

There is a spread of relative coordinate system shifts, and the largest spread occurs between run periods (where the largest typical spread is around $3 \mathrm{~mm}$ ). This is likely a consequence of repositioning the FCal cryostat and/or the movement of the BPCs on the moveable table. A test confirmed that a misalignment of $3 \mathrm{~mm}$ in the coordinate system alignment had no effect on the total clustered energy. The mean value from each position and coordinate was taken as the final alignment between coordinate systems and was used in the cylinder clustering algorithm. These final 


\begin{tabular}{|r|r|}
\hline Position and Coordinate & Mean Shift [mm] \\
\hline \hline $4 \mathrm{~L}, \mathrm{x}$ & $254.7 \pm 2.8$ \\
$4 \mathrm{~L}, \mathrm{y}$ & $1.8 \pm 0.3$ \\
\hline $4 \mathrm{H}, \mathrm{x}$ & $254.6 \pm 2.1$ \\
$4 \mathrm{H}, \mathrm{y}$ & $3.6 \pm 0.2$ \\
\hline $3, \mathrm{x}$ & $139.7 \pm 1.1$ \\
$3, \mathrm{y}$ & $2.7 \pm 1.3$ \\
\hline $2, \mathrm{x}$ & $109.6 \pm 1.1$ \\
$2, \mathrm{y}$ & $2.7 \pm 0.5$ \\
\hline $1, \mathrm{x}$ & $78.7 \pm 0.7$ \\
$1, \mathrm{y}$ & $2.9 \pm 0.5$ \\
\hline
\end{tabular}

Table 3.4: The mean shift between the cluster center coordinates and the BPC track projection coordinates, taken as an average over all runs and particle types for the highest energy beams, for each impact point.

values are quoted in Table 3.4 for each impact position. The errors quoted are the standard deviation of the fitted Gaussian distributions.

In cylindrical clustering the track reconstruction using the BPCs was performed for each event. The projection of this track onto the front face of each FCal module defined the impact position of the particle, and was used as the center of the cylinder. This is an ideal clustering algorithm and takes advantage of additional upstream detector information; unfortunately it will not be possible to use this method for ATLAS data, as there are no tracking detectors in front of the FCal.

\subsubsection{Topological Clustering}

Topological clustering is a method that uses information about the ratio of the channel energy to the expected noise. It is one of two default clustering algorithms used in ATLAS, and the only one used in the FCal. It is instructive to use this algorithm with a known data sample, such as the one from this test beam, and to compare it to alternative clustering algorithms such as the cylindrical clustering method described 
above.

A channel with energy $E$ and noise $\sigma_{\text {noise }}$ has a significance defined as:

$$
\frac{|E|}{\sigma_{\text {noise }}} .
$$

The decision to include a channel in a cluster is based on this significance. In ATLAS the noise will have contributions from electronic noise and pileup (added in quadrature). The test beam provides a simpler environment with single particles, so only electronic noise is considered in this analysis. The electronic noise varied between channels and runs (Section 3.6.2) so the input noise used for the topological clustering was evaluated run-by-run and channel-by-channel using the OF reconstructed random triggered events. The absolute value of the energy was used to remove biases that might arise from including only positive energy channels from noise fluctuations.

The starting point in this method is the identification of seed channels, which were those channels that have a significance greater than some minimum value $S$. The choice of the parameter $S$ is essentially arbitrary, but should make use of the expected energy density within a calorimeter channel for particle showers. It should be large enough to initiate sufficient true clusters to include all of the energy deposited by the incoming particle, but not too small as to cause cluster formation with channels that have purely noise contributions.

All channels that meet this seed requirement are identified as proto-cluster seeds. In the next step the clusters are grown by examining the neighbouring channels of all proto-cluster seed channels. If a neighbour channel has a significance of $N$, then that channel is included in the cluster. The realistic requirement is that $S \geq N$. The clusters continue to expand by an iterative process examining the energy of neighbouring channels. 


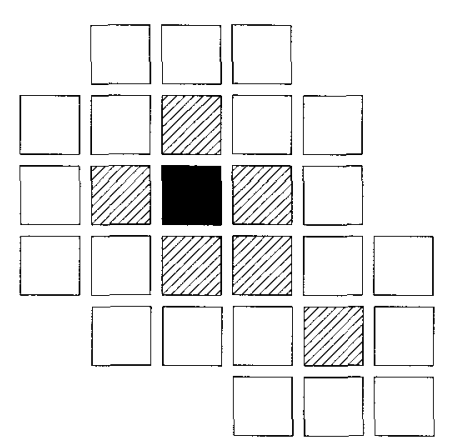

seed

neighbour

perimeter

Figure 3.22: An example of a two-dimensional cluster formed using the topological clustering algorithm. Clusters are seeded by seed channels (filled black boxes), expanded by neighbour channels (dashed boxes), and finalized by including perimeter channels (white boxes).

To ensure tails of the shower are fully included a final check of the perimeter of channels surrounding the outer-most channels already in the cluster. The channels neighbouring the outer-most clustered channels are only included as the perimeter to the cluster if they have a significance greater than $P$. If a neighbour or perimeter channel is included in more than one proto-cluster the proto-clusters in contact via this neighbour or perimeter channel are merged into one cluster.

For the FCal in ATLAS the default cuts on each cluster decision are: $S / N / P=$ $4 / 2 / 0$. As $P=0$ all channels in physical contact with the neighbour channels are included in the cluster. With this set of parameters only $0.006 \%$ of the time are clusters seeded by channels with large noise fluctuations. Variations of these parameters are studied in Section 3.9.1.

Figure 3.22 shows an example of a topological cluster algorithm that has been seeded by one channel in the center, and expanded by six neighbour channels. All channels in geometric contact with a neighbour channel are included in the cluster $(P=0)$.

Two dimensional neighbour channels are those channels that are in physical con- 
tact with each other (including corners) in the same layer. In the FCal this typically means each channel has eight neighbours. The shower expands longitudinally by defining channel neighbours in the previous and next layer that are physically touching and pointing back to the IP. This three dimensional topological clustering is used in the analysis for hadrons, whereas for electrons only the energy clustered in FCal1 is considered (as with the cylindrical clustering).

The default topological clustering algorithm used by ATLAS also activates a cluster splitter that finds local maximum within clusters and splits them, to prevent multiple showers and particles that may overlap forming one giant cluster. It is also used to distinguish electromagnetic and hadronic showers based on cluster shower shape variables. In this test beam analysis the topological splitting algorithm is investigated in Section 3.9.3.

The topological clustering technique attempts to contain all of the energy deposited by the incoming particle and its associated shower particles. Shower development is stochastic in nature, hence the final clusters have no fixed size, either in geometry or in the number of channels clustered. The topological method aims to suppress contributions from noise by requiring a certain significance of the energy to the noise. By limiting the channels clustered based on their signal to noise ratio there is a possibility that not all physical energy deposition will be collected. In ATLAS there are additional corrections for energy outside of the topological cluster, that is calibrated using MC predictions. These additional corrections were not applied in this analysis, but the variables that go into their determination will be studied (Section 3.9.2) and compared between data and MC (Section 3.10.2). 


\subsection{Intrinsic Response of the Forward Calorimeter}

The test beam impact position 4L (from Figure 3.7) is located such that particles traverse minimal upstream material, and the resulting showers are fully contained in the FCal, both in the transverse and longitudinal planes. The following sections use this position to find the intrinsic response of the FCal to electrons and hadrons at a range of energies from approximately $10 \mathrm{GeV}$ to $200 \mathrm{GeV}$.

\subsubsection{Electron Analysis}

Using the channel reconstruction and cylindrical clustering described in the previous sections the channels in FCall were clustered in a cylinder of radius $80 \mathrm{~mm}$ about the cluster center, as defined by the BPC track projections. The energy distribution for all electrons with a beam energy of $193.1 \mathrm{GeV}$ is shown as the solid line in Figure 3.23. There are several peaks in this distribution resulting from different particle contributions in the beam. The lowest energy peak centered at approximately 0 is due to muon contamination. The elongated peak in the middle of the energy distribution, centered around $100 \mathrm{GeV}$ is from hadron contamination in the electron beam. Recall hadrons tend to have a lower response in a non-compensating calorimeter. Also, the channels clustered are in FCall only and hadron showers deposit a significant fraction of energy in the hadronic calorimeters. The highest energy peak at about $190 \mathrm{GeV}$ is the main electron peak of interest.

The data were cleaned by the standard beam cleaning procedure, as described in Section 3.5.1, as well as some additional complex beam cleaning cuts defining the envelope of the electron beam (versus hadrons) using the position and track slope

of the particles, to further separate hadrons from electrons [19]. After the data selection was applied the resulting energy distribution was that represented by points 


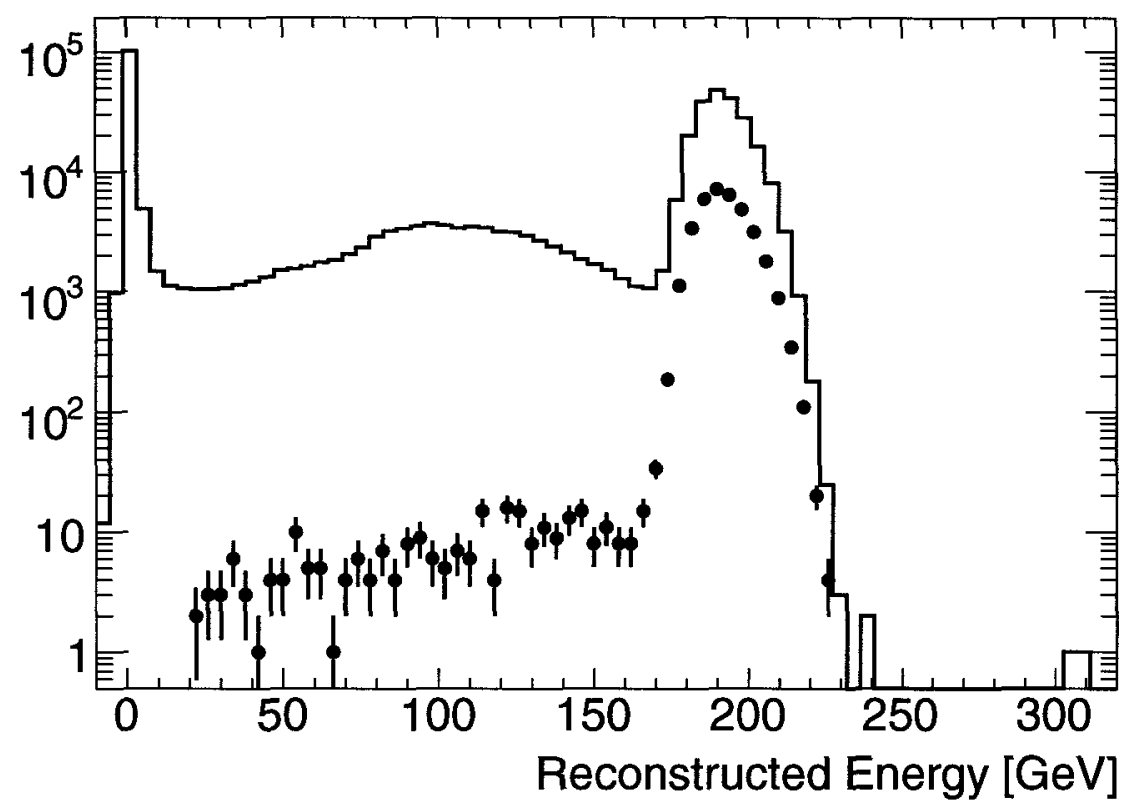

Figure 3.23: $193.1 \mathrm{GeV}$ electron data in position $4 \mathrm{~L}$ with various cuts applied. The solid black line represents all of the triggered events without any events cuts applied, while the data represented by points with error bars are the data after applying all event selection criteria.

in Figure 3.23. Table 3.5 shows the final number of events after all cuts for each beam energy in the position $4 \mathrm{~L}$ electron analysis.

The final electron energy distribution in Figure 3.23 is a relatively clean sample of electrons, with some contamination visible in the low energy tail. For this particular case it might be possible to define the mean energy as the mean value of the distribution. However not all beam energies yield such a clean sample, hence a fitting procedure has been developed to extract the signal peak (electron) and standard deviation of the energy distributions for a single beam energy.

\begin{tabular}{|r|c|c|c|c|c|c|c|c|}
\hline Beam Energy [GeV] & 10 & 20 & 40 & 60 & 80 & 100 & 147.8 & 193.1 \\
\hline \hline Number of Events & 41270 & 84067 & 29125 & 2275 & 26919 & 28829 & 14328 & 35721 \\
\hline
\end{tabular}

Table 3.5: The total number of events after all cuts for the $4 \mathrm{~L}$ electron analysis. The CEDAR trigger was used for the $60 \mathrm{GeV}$ electron beam energy. 
The first step in the procedure involves fitting the distribution to a single Gaussian function, where the range of the fit is limited to include the electron peak (to avoid fits to the hadron or muon peak at lower energies). This gives a first rough approximation for the mean position and width of the distribution. This fit was used as input to constrain the second fit, which is the sum of two Gaussian distributions. The data are best described by a double Gaussian, with one Gaussian centered on the main peak, approximately at the single Gaussian mean, and the second Gaussian encompassing the high energy tail. There is a small high energy tail in the energy distribution for the FCal, resulting from the slight impact point dependence of the detector response. When a particle interacts first with the liquid argon gap it produces a higher response, compared to when it hits the absorber first [26]. The fraction of the detector that is liquid argon is quite small $(\sim 1 \%)$, so there is a small fraction of particles that will first interact with the liquid argon gap, leading to few events in the high energy tail.

The mean value of the reconstructed energy is the first moment (expectation value) of the energy in the double Gaussian distribution with parameters defined by the fit:

$$
\langle E\rangle=\frac{\int E G(E) d E}{\int G(E) d E}
$$

where $G(E)=G_{1}(E)+G_{2}(E)$, and $G_{1}(E)$ and $G_{2}(E)$ are the two Gaussian functions, each with three fit parameters (normalization, mean, and standard deviation). The standard deviation of the distribution is calculated by:

$$
\sigma_{E}=\sqrt{\left\langle E^{2}\right\rangle-\langle E\rangle^{2}}
$$

where $\left\langle E^{2}\right\rangle$ is the second moment of the energy with the double Gaussian function. The error associated with the mean is $\sigma_{\langle E\rangle}=\sigma_{E} / \sqrt{n}$, where $n$ is the number of events 


\begin{tabular}{|r|c|c|}
\hline Beam Energy [GeV] & Mean $[\mathrm{GeV}]$ & $\sigma[\mathrm{GeV}]$ \\
\hline \hline 10 & $9.236 \pm 0.007$ & $1.464 \pm 0.005$ \\
20 & $19.098 \pm 0.006$ & $1.801 \pm 0.004$ \\
40 & $39.353 \pm 0.015$ & $2.485 \pm 0.010$ \\
60 & $58.887 \pm 0.078$ & $3.136 \pm 0.055$ \\
80 & $78.870 \pm 0.025$ & $3.974 \pm 0.017$ \\
100 & $98.816 \pm 0.029$ & $4.724 \pm 0.020$ \\
147.8 & $146.729 \pm 0.063$ & $6.322 \pm 0.045$ \\
193.1 & $191.675 \pm 0.042$ & $7.792 \pm 0.029$ \\
\hline \hline
\end{tabular}

Table 3.6: The mean and standard deviation of the electron energy distributions in position $4 \mathrm{~L}$. The errors are statistical only.

under the double Gaussian distribution. The error associated with the standard deviation is $\sigma_{\sigma_{E}}=\sigma_{E} / \sqrt{2 n}$.

The energy distributions for all electron beam energies in position $4 \mathrm{~L}$ are shown in Figure 3.24, superimposed are the fits to the double Gaussian function. The final mean and standard deviation of the electron peak in the energy distributions are shown in Table 3.6.

The data can be used to ensure that the electromagnetic response of the detector is linear, meaning that the conversion between the reconstructed peak height in ADC and energy in $\mathrm{GeV}$ is constant across the range of all relevant particle energies. The predicted conversion constant was calculated by J. Rutherfoord using SPICE simulations of the FCal electronics chain. For each module the conversion from units of ADC to $\mathrm{GeV}$ was predicted to be:

FCal1 $: 0.0833 \mathrm{GeV} /$ ADC FCal2 $: 0.1639 \mathrm{GeV} /$ ADC $\quad$ FCal3 $: 0.1852 \mathrm{GeV} /$ ADC .

This means for a channel in FCall a reconstructed pulse height of 12 ADC counts corresponds to $1 \mathrm{GeV}$ of energy deposition. These predicted conversion constants 

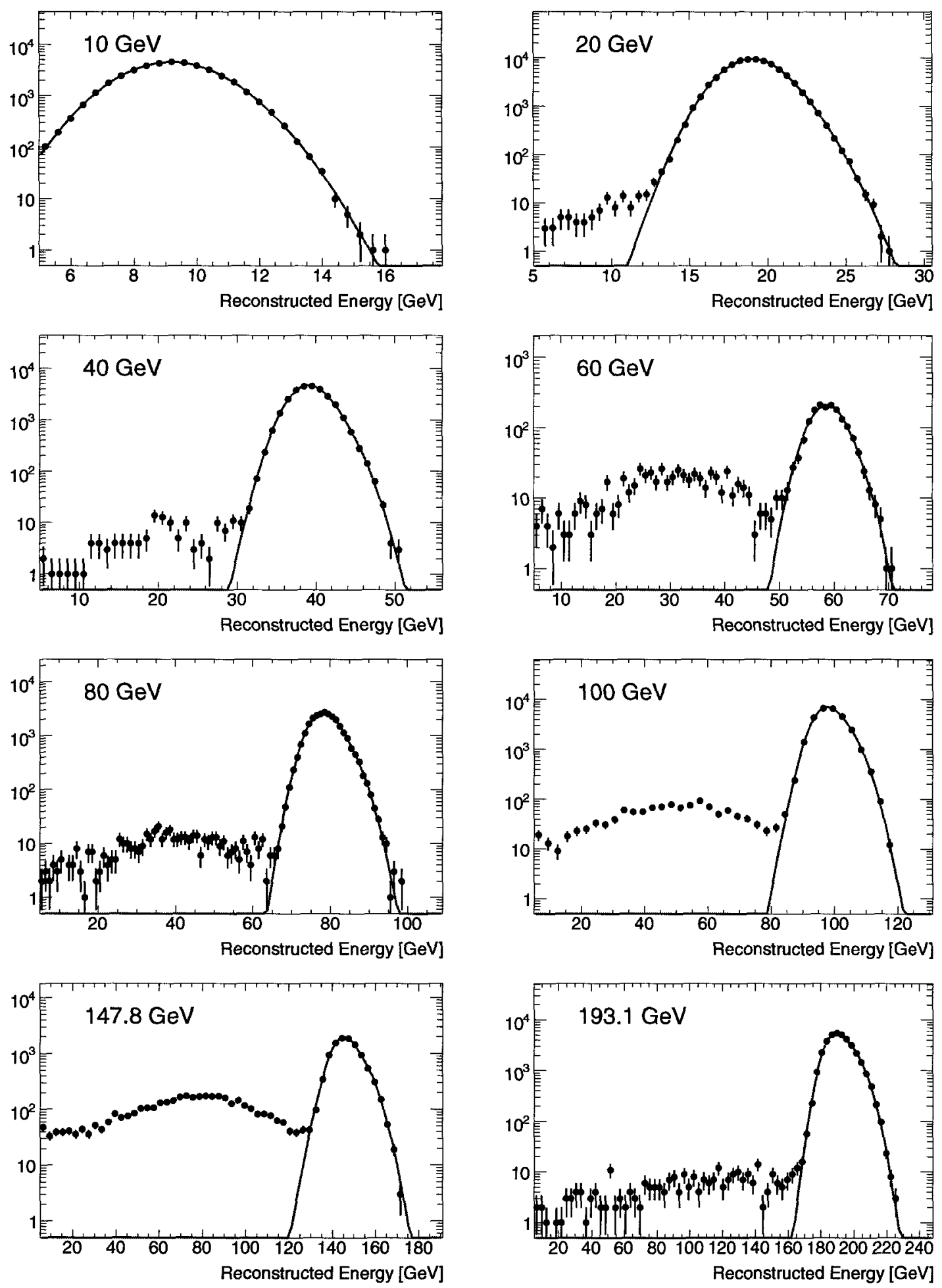

Figure 3.24: Electron energy distributions, superimposed with double Gaussian fits, at position 4L. The real beam energy is indicated on each plot in the upper left corner. 


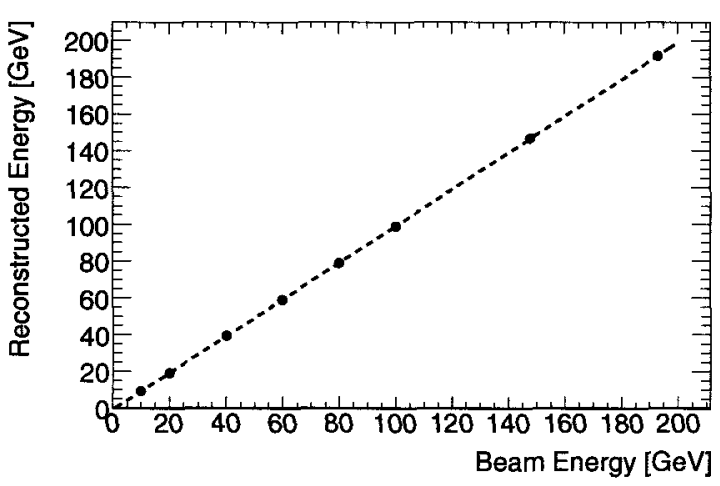

(a)

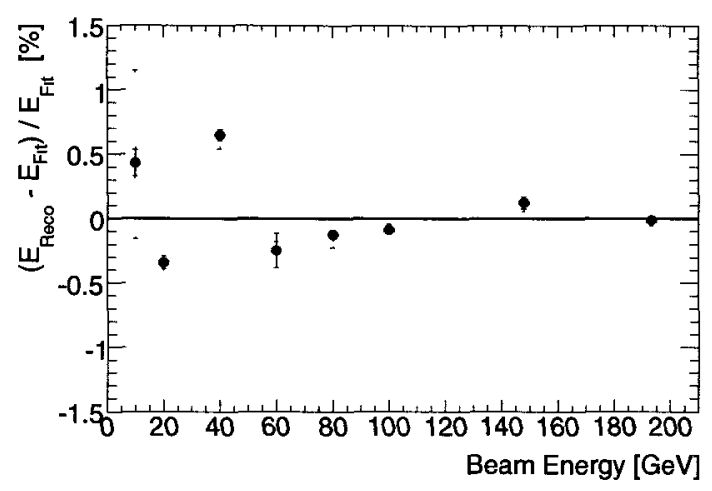

(b)

Figure 3.25: The reconstructed energy versus the beam energy with a linear fit to the data. The residual shows the percent difference between the reconstructed energy $\left(E_{\text {Reco }}\right)$ and the fit energy $\left(\mathrm{E}_{\mathrm{Fit}}\right)$. The error bars on the residual reflect the statistical errors, and the dashed lines represents the systematic uncertainties.

have been applied to the data.

The electron data can be used to test the predicted conversion in FCall by performing a linear fit to the mean reconstructed energy as a function of the beam energy. This fit should yield a slope with value unity, within errors. The plot of this linearity is shown in Figure 3.25(a). Superimposed is a linear fit to the data points, where both the slope and intercept are free parameters. To verify that the response is linear across all energy points a plot of the residual to the linear fit is shown in Figure 3.25(b). The dashed error bars on the points in Figure 3.25(b) represent the systematic errors.

There are two dominant systematic errors contributing to the linearity and residuals. The first arises from the uncertainty of the beam energy and composition delivered to the $\mathrm{H} 6$ experimental hall. The source of the low energy beams $(40 \mathrm{GeV}$ and less) was a tertiary beam, whereas the higher energy beams originated from a secondary beam. The lowest energy points supplied by the tertiary beam have the largest deviation from the fit.

There should be a very small (and negligible) difference of the response of any 
calorimeter to electrons versus positrons of the same energy. The test beam used a combination of electrons and positrons at different energies. The choice of particle type was tied to the demands of other experiments in neighbouring test areas. The $10 \mathrm{GeV}$ and $20 \mathrm{GeV}$ beam energies contained runs of mixed electrons and positrons, whereas data for all other beam energies were of either solely electrons or positrons (and associated beam contamination). The energy distributions for the $10 \mathrm{GeV}$ and $20 \mathrm{GeV}$ electron and positron data were fit separately and compared to the combination. The fit results to these subsets are shown in Table 3.7. These results indicate that there was a difference in the response to electrons and positrons. Since there is no physical motivation why a calorimeter would behave this way, it is assumed the difference is due to the supplied beam. In changing polarity the accelerator magnets needed to be degaussed and retuned, potentially causing a systematic difference observed in the results. Different combinations of beam composition were used to refit for the linearity. The largest difference between the refitted residuals and the nominal result (which uses the fit to all the data) was taken as the systematic uncertainty on the residual associated to the beam energy and composition. This was calculated for each beam energy point. It is possible that similar effects are present for the singly charged beams, but with only one type of particle it is not possible to quote an uncertainty of this type with these points.

The second dominant source of systematic error on the linearity was the choice of an $80 \mathrm{~mm}$ cylinder radius for the clustering; cylindrical clustering with radii of $120 \mathrm{~mm}$ and $160 \mathrm{~mm}$ was also studied, the results of which are shown in Table 3.8. As the radius of the cylinder used for the clustering was increased from $80 \mathrm{~mm}$ to $120 \mathrm{~mm}$ to $160 \mathrm{~mm}$ there was slightly more energy clustered (by about $1 \%$ at beam energies of $193.1 \mathrm{GeV}$ ); however there was a large increase in the width of the distribution (about $4 \%$ at $193.1 \mathrm{GeV}$ ). As previously described, one important requirement for 


\begin{tabular}{|c|l|c|c|}
\hline Beam Energy [GeV] & Particle (No. evts) & Mean $[\mathrm{GeV}]$ & $\sigma[\mathrm{GeV}]$ \\
\hline \hline \multirow{2}{*}{10} & $e-(19937)$ & $9.151 \pm 0.010$ & $1.462 \pm 0.007$ \\
& $e+(21333)$ & $9.314 \pm 0.010$ & $1.458 \pm 0.007$ \\
& combined (41270) & $9.236 \pm 0.007$ & $1.464 \pm 0.005$ \\
\hline \multirow{2}{*}{20} & $e-(38275)$ & $18.994 \pm 0.009$ & $1.793 \pm 0.006$ \\
& $e+(45792)$ & $19.184 \pm 0.008$ & $1.795 \pm 0.006$ \\
& combined (84067) & $19.098 \pm 0.006$ & $1.801 \pm 0.04$ \\
\hline \hline
\end{tabular}

Table 3.7: The reconstructed mean for each subset of runs (electrons versus positrons), and a percent difference from the current linearity fit and the beam energy. The values in brackets represent the default values, when all the data at each energy point is used (not distinguishing the particle charge). The errors are statistical uncertainties only.

\begin{tabular}{|r|r|c|c|c|}
\hline $\begin{array}{r}\text { Beam Energy } \\
\text { GeV }\end{array}$ & \multicolumn{2}{|c|}{$120 \mathrm{~mm}$ Cylinder } & \multicolumn{2}{c|}{$160 \mathrm{~mm}$ Cylinder } \\
& Mean $[\mathrm{GeV}]$ & $\sigma[\mathrm{GeV}]$ & Mean $[\mathrm{GeV}]$ & $\sigma[\mathrm{GeV}]$ \\
\hline \hline 10 & $9.314 \pm 0.010$ & $2.050 \pm 0.007$ & $9.376 \pm 0.013$ & $2.647 \pm 0.009$ \\
20 & $19.283 \pm 0.008$ & $2.299 \pm 0.006$ & $19.314 \pm 0.010$ & $2.895 \pm 0.007$ \\
40 & $39.724 \pm 0.017$ & $2.839 \pm 0.012$ & $39.809 \pm 0.020$ & $3.354 \pm 0.014$ \\
60 & $59.240 \pm 0.101$ & $4.072 \pm 0.071$ & $59.007 \pm 0.110$ & $4.503 \pm 0.078$ \\
80 & $79.557 \pm 0.027$ & $4.310 \pm 0.019$ & $79.657 \pm 0.055$ & $4.929 \pm 0.022$ \\
100 & $99.738 \pm 0.030$ & $4.971 \pm 0.021$ & $99.924 \pm 0.033$ & $5.520 \pm 0.024$ \\
147.8 & $147.996 \pm 0.065$ & $6.536 \pm 0.046$ & $148.217 \pm 0.069$ & $6.896 \pm 0.049$ \\
193.1 & $193.324 \pm 0.042$ & $7.883 \pm 0.030$ & $193.714 \pm 0.043$ & $8.115 \pm 0.031$ \\
\hline \hline
\end{tabular}

Table 3.8: The reconstructed mean energy and standard deviation for electrons of different energies, using a $120 \mathrm{~mm}$ and $160 \mathrm{~mm}$ radius for clustering the FCall channels.

a calorimeter is good energy resolution. The larger the cylinder radius, the more channels without real energy are clustered, degrading the energy resolution. The topic of energy resolution is discussed in the following pages.

Other sources of systematic errors that were studied were the technique for fitting the mean and width of the energy distributions, variations of the fit limits, bin widths, and modelling of the hadron peak.

The final linear fit to the electron data yielded a slope of 0.997 and an intercept of $0.771 \mathrm{GeV}$. The negative energy intercept reflects missing energy that could be caused 
by energy losses in upstream materials. Although position 4L had a minimal amount of upstream material, beam particles still travelled through some material, including the various upstream detectors and the cryostat wall. MC simulations (described in Section 3.10) of the test beam setup predicted energy losses of $0.3 \mathrm{GeV}$ at $10 \mathrm{GeV}$ and $1.1 \mathrm{GeV}$ at $193.1 \mathrm{GeV}$ for electrons in the upstream beam materials present for position $4 \mathrm{~L}$ [27]. If this loss was taken into account the effect would be to increase the slope closer to unity and move the intercept closer to zero. To test for possible leakage into the other modules, the analysis was run including the sum of all the energy in FCal2 and FCal3 contained in an $80 \mathrm{~mm}$ cylinder radius, the results were not significantly changed from the above, any changes were mainly due to statistical fluctuations of noise.

Taking into account the systematics described above, the intrinsic electromagnetic energy scale of the conversion between the electronics read out in ADC counts and energy in FCal1 is verified with the test beam data to be within $1 \%$ of the predicted conversion. It is linear within $1.5 \%$ across a range of electron energies from $10 \mathrm{GeV}$ to $193.1 \mathrm{GeV}$, with improved reconstruction at higher energies. The analysis of the electron linearity across the range of test beam energies in FCall confirms the SPICE predicted conversion between the detector response in units of ADC counts and reconstructed energy in units of $\mathrm{GeV}$ for the test beam electronics chain. There are some slight differences in the electronics chain in ATLAS, but validation of the simulation gives confidence in the prediction of the same conversion of the FCal used in the ATLAS environment.

The energy resolution of the FCal is found by taking the standard deviation of the energy distribution and dividing it by the mean reconstructed energy: $\sigma_{E} /\langle E\rangle$. This was done for each of the test beam energy points. The energy resolution function describing the response of the calorimeter to stochastic shower fluctuations and intrinsic 
qualities of the calorimeter is found by fitting this data to Equation 3.12. At some of the beam energies there was increased noise in the electronics chain, and so these energy points have larger contributions from noise. In the standard formula used to parametrize the energy resolution it is assumed that the noise is constant across all energies. To account for this problem, a procedure was developed to subtract the noise from the physics data in the energy resolution.

The total width of the measured energy distribution is:

$$
\sigma_{\mathrm{E}}=\sigma_{\text {total }}=\sigma_{\text {signal }} \oplus \sigma_{\text {noise }}
$$

where $\sigma_{\text {signal }}$ is the width due to the electron energy measurement and $\sigma_{\text {noise }}$ is the contribution from noise. The physics data were fit to a double Gaussian (as described earlier) and the mean and standard deviation were taken from this fit, where the measured width $\left(\sigma_{\text {total }}\right)$ arises from the signal response and the contributions from noise.

To calculate $\sigma_{\text {noise }}$ the standard data reconstruction procedure was applied to the random pedestal data. For every event in the physics data an event was chosen at random from the reconstructed random pedestal data within the same run. The channels that were clustered in the physics data were then clustered in the random pedestal data, forming a noise cluster for that event composed entirely of the same channels. The choice of using all channels from one event in the random data ensured cross-channel correlations of the noise were accounted for. The 'energy' distribution of the noise for those same clustered channels were fit to a Gaussian distribution, the width of which was $\sigma_{\text {noise }}$ (the mean was centered on zero after pedestal subtraction). The distribution of $\sigma_{\text {noise }}$ is shown in Figure 3.26(a) for each of the beam energies; it can be seen in this figure that the $80 \mathrm{GeV}, 100 \mathrm{GeV}$, and $150 \mathrm{GeV}$ energy points 


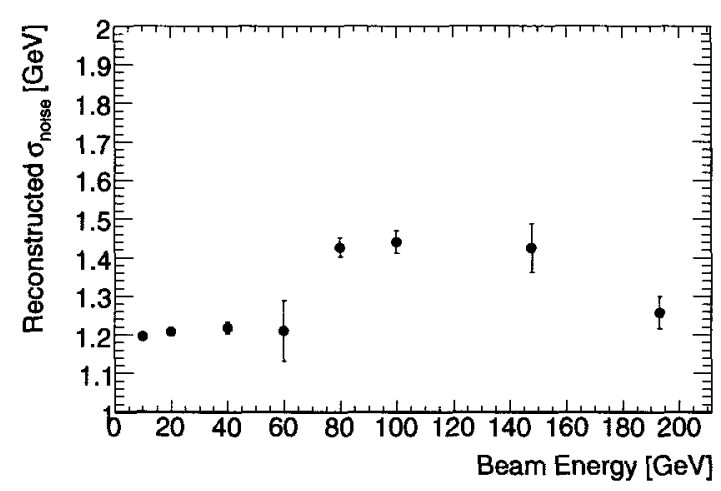

(a)

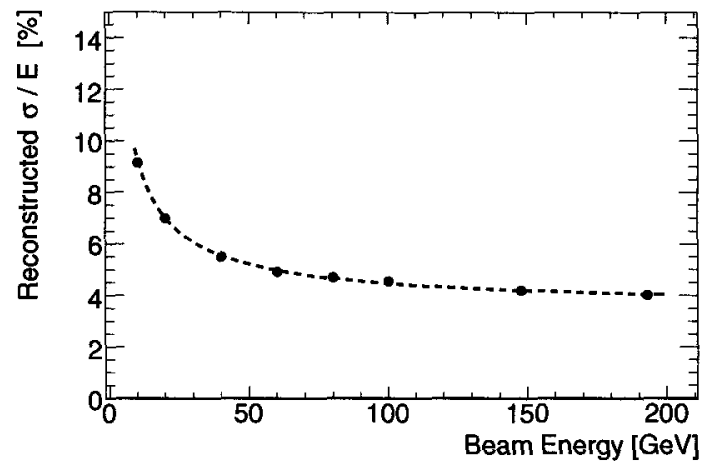

(b)

Figure 3.26: (a) Electronic noise that is subtracted for each beam energy, and (b) the noise-subtracted energy resolution.

have larger noise contributions than that of the other beam energies.

The width of the energy distribution arising from the response of the signal was found by re-arranging Equation 3.26 and solving for $\sigma_{\text {signal }}$. The parameterization of the noise-subtracted energy resolution of the electron signal is then a modified version of Equation 3.12:

$$
\frac{\sigma_{\text {signal }}(E)}{E}=\frac{\sigma(E)}{E}=\frac{a}{\sqrt{E}} \oplus b
$$

where the variable definitions are as before. The noise-subtracted energy resolution for the electron data in position $4 \mathrm{~L}$ is shown in Figure 3.26(b). Fitting these points for the stochastic term $(a)$ and constant term $(b)$ of Equation 3.27 results in:

$$
a=27.0 \pm 1.0 \% \cdot \mathrm{GeV}^{-1 / 2} \text { and } \quad b=3.5 \pm 0.1 \%
$$

Only the systematic errors are quoted as the statistical errors are negligible. The systematic errors considered are those previously described, with the choice of cluster cylinder size the largest contribution to the uncertainty. 


\subsubsection{Hadron Analysis}

The FCal was primarily designed to measure energy from jets and contribute to the measurement of the missing transverse energy. Two aspects of any hadronic calorimeter that are important to understand are its response to hadrons at the electromagnetic scale over a range of energies, and its associated energy resolution. These are studied with the test beam hadron data, along with a simple correction that accounts for energy losses associated with hadronic shower development.

The range of hadron beam energies and associated number of events after all event cuts were applied is shown in Table 3.9. In the analysis of the hadron data there were no cuts applied based on the response of the downstream muon scintillator detector, as there could have been valid decays of pions into muons. The primary beam content for the hadron beam energies were $\pi^{+}$and $\pi^{-}$. However the general case of hadrons is considered here as there was proton contamination in the $\pi^{+}$beams. The CEDAR scintillator was used for the $\pi^{+}$beam energies to reduce the amount of this contamination.

The hadron data studied in this section was taken in position $4 \mathrm{~L}$; the optimal location in the detector for full containment with minimal upstream material. The FCal channels were clustered using the cylinder clustering algorithm, with a cylinder of radius $160 \mathrm{~mm}$. The cylinder centers were taken as the projections on to the front face of each module using the BPC track reconstructions. All channels within these cylinder volumes from FCal1, FCal2, and FCal3 were summed together to get the

\begin{tabular}{|r|c|c|c|c|c|c|c|c|}
\hline Beam Energy [GeV] & 10 & 40 & 60 & 80 & 100 & 120 & 150 & 200 \\
\hline \hline Number of Events & 3648 & 7730 & 77810 & 62268 & 13135 & 84875 & 107404 & 93507 \\
\hline
\end{tabular}

Table 3.9: The total number of events after all cuts for the $4 \mathrm{~L}$ hadron analysis. 
total reconstructed energy of an event.

The energy distributions for the hadron data at the electromagnetic scale are shown in Figure 3.27. The energy distributions were fit to a double Gaussian, as with the electron data, following the same procedure. The results of these fits are superimposed on the figure, and are used to calculate the mean energy and spread of the energy distribution.

The mean reconstructed energy of the hadron beams is much less than the equivalent energy electron beams, due to the differences in shower development and secondary particle content within those showers. The widths of the hadron energy distributions are larger, a reflection of the greater variations in hadronic shower development. A simple hadronic calibration scheme was developed to improve the mean reconstruction energy and the overall energy resolution.

In this procedure one weight was derived for each module and then the reconstructed energy for each module was multiplied by its associated weight. These weights are called flat weights. There are three modules in the experimental setup, so three weights were derived such to minimize the energy resolution with the constraint that the sum of the average reconstructed energy over each module would equal the beam energy. With three weights and one constraint, there were two free parameters in the fit, $w_{1}$ and $w_{2}$. For every event $k$ the amount of energy (at the electromagnetic scale) in the $i$ th module was $E_{\imath, k}$. The function minimized was:

$$
S=\sum_{k=1}^{N_{\text {events }}}\left[\sum_{\imath=1}^{2} w_{\imath} E_{\imath, k}^{r e c o}+\frac{E^{\text {beam }} E_{3, k}^{r e c o}}{\left\langle E_{3}^{r e c o}\right\rangle}-\frac{E_{3, k}^{r e c o}}{\left\langle E_{3}^{r e c o}\right\rangle} \sum_{\imath=1}^{2} w_{\imath}\left\langle E_{\imath}^{r e c o}\right\rangle-E^{\text {beam }}\right]^{2},
$$

where $E^{\text {beam }}$ was the beam energy and $\left\langle E_{\imath}^{r e c o}\right\rangle$ was the mean energy reconstructed in 

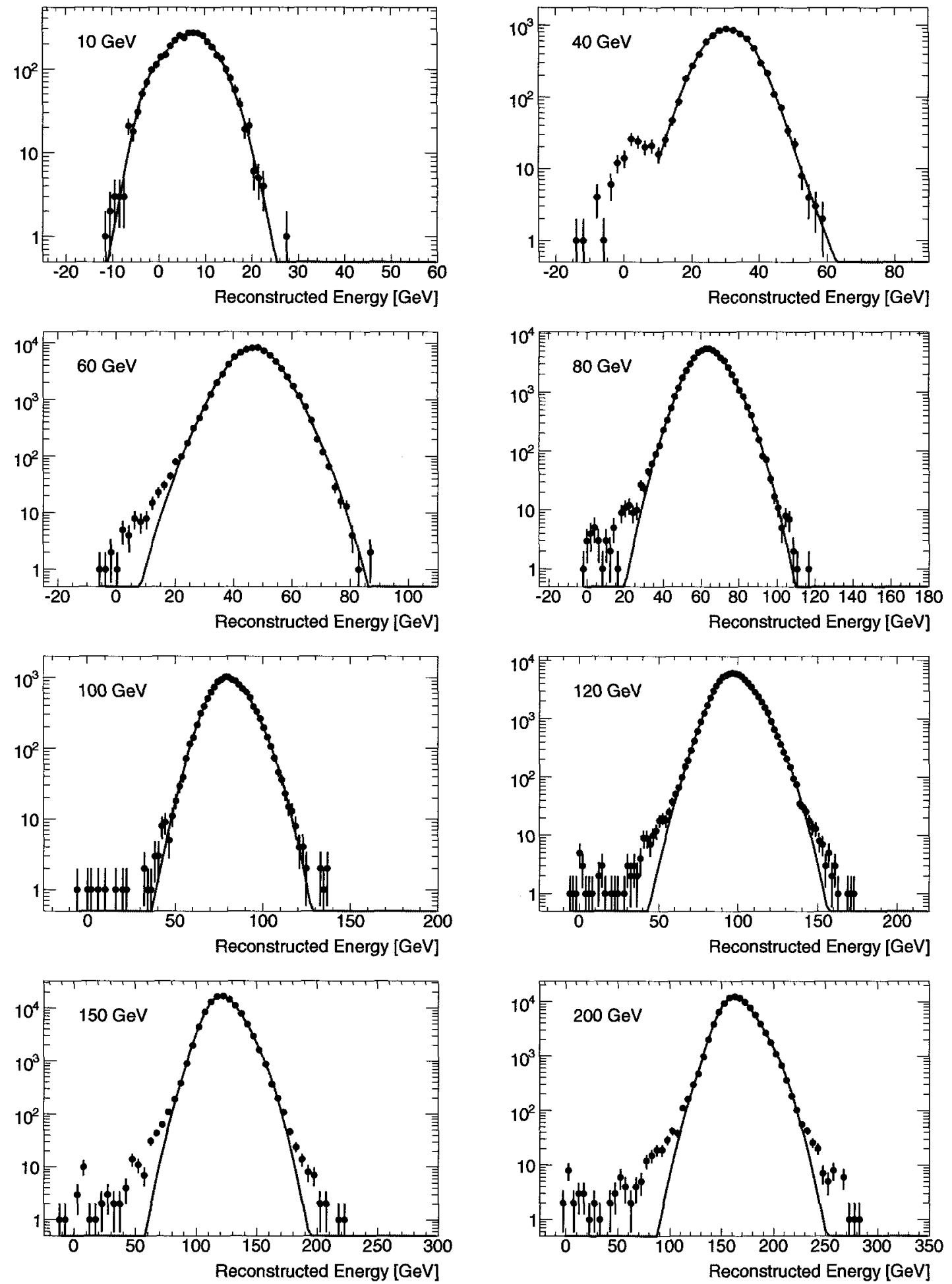

Figure 3.27: Hadron energy distributions in position $4 \mathrm{~L}$, superimposed with the double Gaussian fits used to calculate the mean and standard deviation. The real beam energy is indicated on each plot at the upper left corner. 


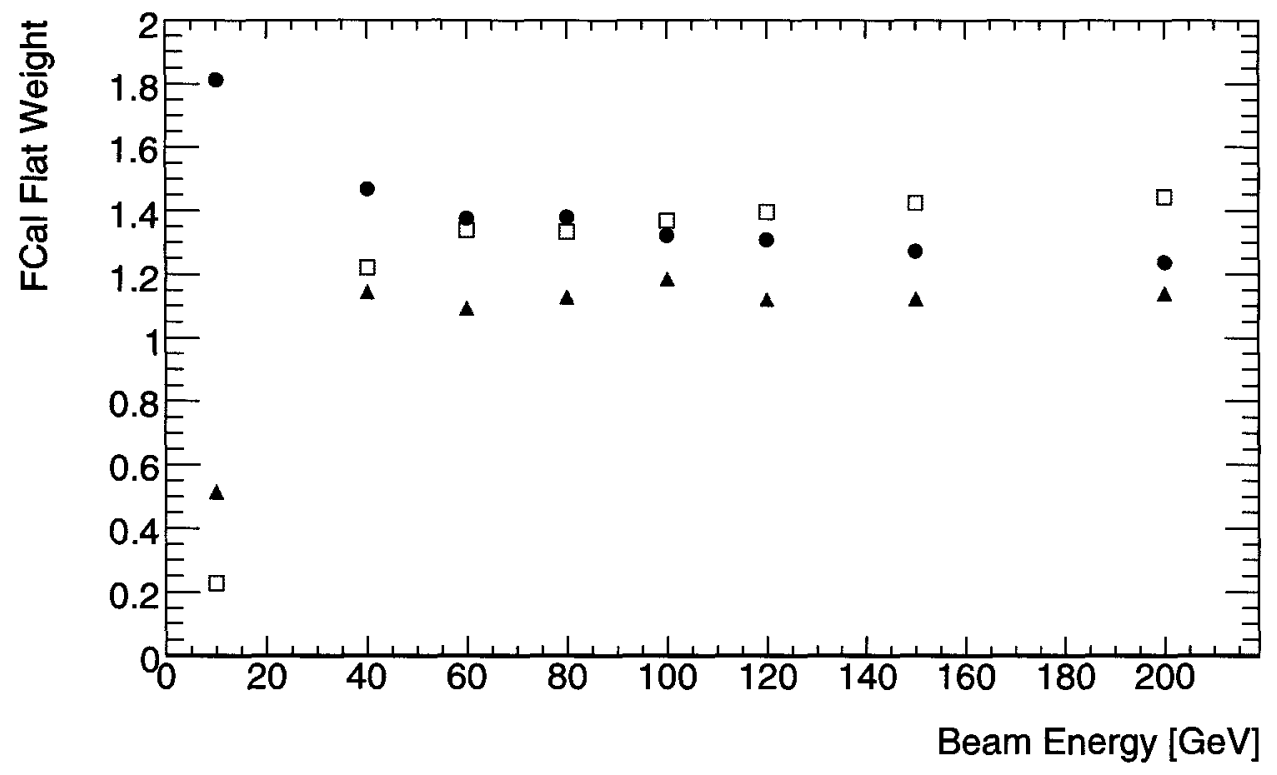

Figure 3.28: Flat weights for each FCal module extracted at each of the beam energies, at position $4 \mathrm{~L}$. The weights for FCal1 are shown as the filled circles, the weights for FCal2 as the filled triangles, and the weights for FCal3 as the open squares.

the $i$ th module. The fit results for $w_{1}$ and $w_{2}$ were then used to calculate $w_{3}$ :

$$
w_{3}=\frac{E^{\text {beam }}}{\left\langle E_{3}^{\text {reco }}\right\rangle}-\frac{1}{\left\langle E_{3}^{\text {reco }}\right\rangle} \sum_{\imath=1}^{2} w_{\imath}\left\langle E_{\imath}^{\text {reco }}\right\rangle
$$

This calibration does not account for leakage from the back of the calorimeter. An improvement to this technique would be to calibrate the downstream tail catcher and include the energy deposited in this detector. The weights were extracted for each module and for each of the eight beam energies. These fit results are shown in Figure 3.28. For the higher energy beam energies the extracted weights are similar. At the lowest beam energy, $10 \mathrm{GeV}$, the weights in each module are quite different from at other energies. This is because at such a low energy there was very little particle energy deposited in FCal2 and FCal3 (approximately 1\% of the total energy clustered is in FCal3 for the $10 \mathrm{GeV}$ beam), reflected in weights below one, suppressing mostly the noise contributions in the clustered channels. 
ATLAS does not encourage the application of energy dependent calibration schemes because of computing constraints; for these flat weights the choice was made to apply the weights extracted with the $200 \mathrm{GeV}$ beam. The energy reconstructed in FCal1, FCal2, and FCal3 (in units of GeV) was then multiplied by the flat weights of 1.238 , 1.140 , and 1.444, respectively. These distributions were then fit to a double Gaussian; the mean and standard deviation of this distribution for each beam energy with the $200 \mathrm{GeV}$ weights are listed in Table 3.10 . At $200 \mathrm{GeV}$ the mean reconstructed energy is $200.108 \mathrm{GeV}$, the weights are extracted such that the mean of the raw distribution equals $200 \mathrm{GeV}$, where as the values listed in the table stem from the fit of the data to a double Gaussian.

\begin{tabular}{|r|c|c|}
\hline Beam Energy $[\mathrm{GeV}]$ & Mean $[\mathrm{GeV}]$ & $\sigma[\mathrm{GeV}]$ \\
\hline \hline 10 & $8.251 \pm 0.108$ & $6.505 \pm 0.077$ \\
40 & $37.403 \pm 0.096$ & $8.352 \pm 0.068$ \\
60 & $57.020 \pm 0.034$ & $9.583 \pm 0.024$ \\
80 & $77.492 \pm 0.046$ & $11.588 \pm 0.033$ \\
100 & $97.813 \pm 0.116$ & $13.289 \pm 0.082$ \\
120 & $118.524 \pm 0.049$ & $14.353 \pm 0.035$ \\
150 & $148.683 \pm 0.050$ & $16.268 \pm 0.035$ \\
200 & $200.108 \pm 0.065$ & $19.820 \pm 0.046$ \\
\hline \hline
\end{tabular}

Table 3.10: The mean and standard deviation of the hadron energy distributions in position $4 \mathrm{~L}$. The errors are statistical uncertainties only.

The response of a calorimeter to hadronic showers is not linear across energies, and depends on the fraction of the electromagnetic component which increases with incoming particle energy. Figure 3.29 demonstrates the non-linearity of hadronic showers in the FCal and emphasizes the challenges with this weighting scheme; in this figure the flat weights have been applied to the data, therefore the response to $200 \mathrm{GeV}$ beams is approximately one.

The final noise-subtracted energy resolution for hadrons in position $4 \mathrm{~L}$ with the flat 


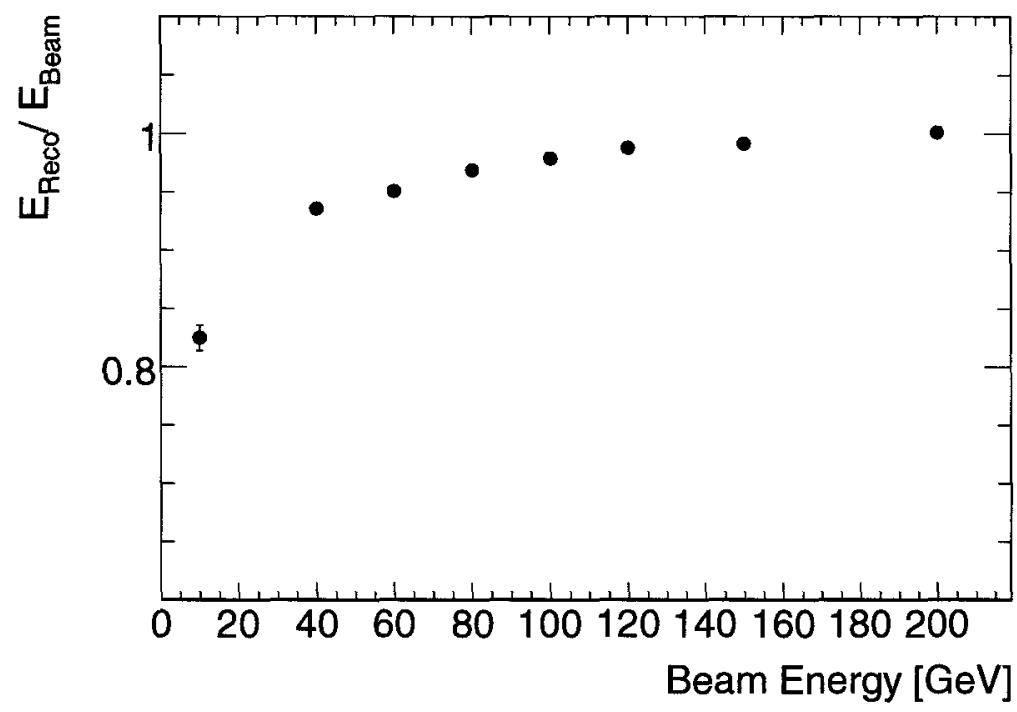

Figure 3.29: Hadron linearity with the flat weight hadronic scheme applied, at position $4 \mathrm{~L}$. $\mathrm{E}_{\text {Reco }}$ is the reconstructed energy and $\mathrm{E}_{\text {Beam }}$ is the beam energy.

hadronic weighting scheme applied is in Figure 3.30(b). The noise that is subtracted in quadrature from each beam energy point can be seen Figure 3.30(a); the flat weights have also been applied to the noise. The largest source of systematic error was the choice of flat weight to apply. The flat weights derived at the highest four energies were applied to the data, and the difference was taken as the systematic uncertainty. The energy resolution parameters achieved for the test beam hadron data at position $4 \mathrm{~L}$ with the simple hadronic weight scheme are a sampling term of $86.5 \pm 3.6$ (sys) $\% \cdot(\mathrm{GeV})^{-1 / 2}$ and a constant term of $7.2 \pm 1.1$ (sys) \%. Symmetric systematic errors are quoted, as the analysis used the highest energy beam available, and so it is assumed that equivalent errors would be observed if higher energy beam energies were used to extract the weights.

Other studies have shown that the use of more sophisticated hadronic calibration schemes that make use of the transverse segmentation of the FCal can significantly improve the energy resolution $[19,28]$. 


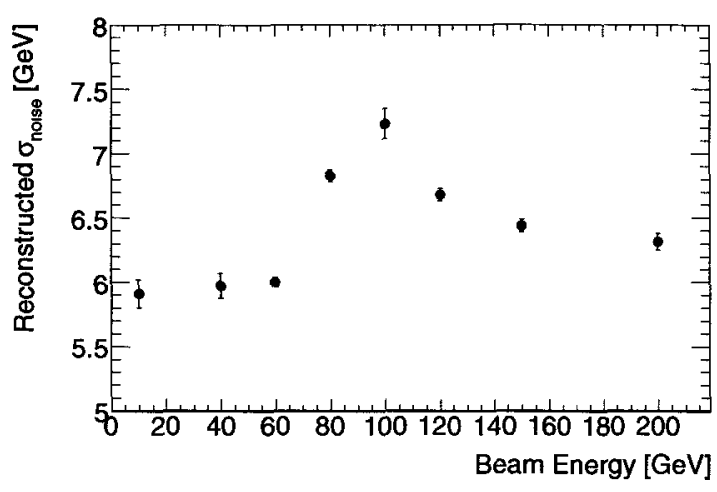

(a)

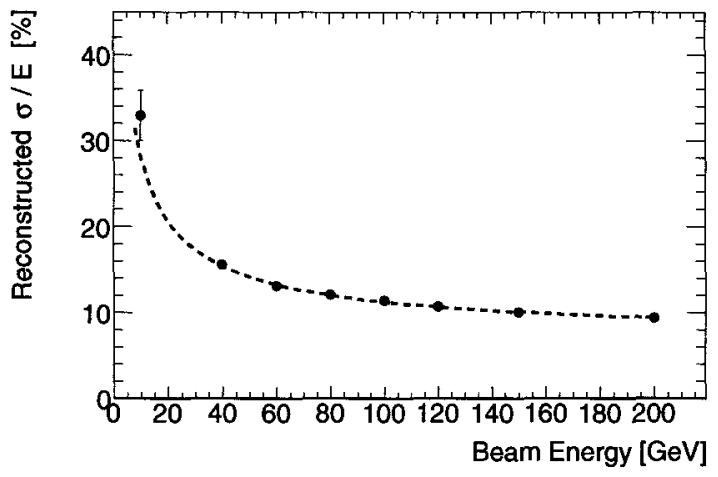

(b)

Figure 3.30: The noise subtracted from each beam energy in (a) and in (b) the noisesubtracted energy resolution for hadrons with flat weights applied in position 4L. In figure (b) the points have been fit to the noise-subtracted energy resolution Equation 3.27.

\subsubsection{Correction to Previous Published Results}

The results of the intrinsic response of the FCal to electrons and hadrons using cylindrical clustering were previously published $[19,29]$. The results presented in this thesis and in the papers cited used the same ATLAS software framework and analysis code. However in the preparation of this thesis a small bug was discovered in the software analysis.

One goal of the analysis was to verify the conversion between detector readout in ADC counts and energy for FCal1. In the initial analysis presented in the cited papers this was achieved by running the analysis chain in units of ADC counts and plotting the electron linearity in FCal1 as the reconstructed detector response in ADC counts versus beam energy in $\mathrm{GeV}$. After the signal pulse peak had been reconstructed with the OF technique, the software analysis applied a conversion between the detector response and energy in units of $\mathrm{MeV}$; in the original analysis this was set to one, to essentially keep the units in ADC counts. For computing speed and memory constraints this peak value was then cast as an integer, essentially truncating the numerical precision after the decimal point. If a channel was reconstructed with a 
peak height of 32.85 in Arbitrary Units (AU) then in the casting it to an integer the peak value would be $32 \mathrm{AU}$. This number would then propagate through the rest of the analysis chain, and from a user's perspective the final reconstructed energy in the channel would be $32 \mathrm{AU}$. The software package that does this truncation was originally used with units of $\mathrm{MeV}$, hence at most the final reconstructed channel energy would be too low by a maximum of $1 \mathrm{MeV}$. This was not the case when the software was used for the original FCal pulse reconstruction. In the original test beam analysis done in units of ADC counts, the truncation of trailing decimal places led to a loss of reconstructed energy of a maximum of 1 ADC. Unfortunately this translates to a maximum loss of $83.3 \mathrm{MeV}, 163.29 \mathrm{MeV}$, and 185.2 MeV for FCal1, FCal2, and FCal3, respectively, per channel (using the conversions in Equation 3.8.1). The more channels included in the cluster with real energy deposition (versus noise) the larger the impact.

For this thesis the choice was made to re-reconstruct the test beam data in units of $\mathrm{MeV}$, hence the quoted values for the intrinsic response vary slightly from the cited published results, the consequence of which was that on average more energy was reconstructed for each beam energy. For electrons the difference was minimal and within the quoted errors. For hadrons this effect was larger, as more channels are clustered (160 mm cylinder radius), and included channels in FCal2 and FCal3 that have a larger $\mathrm{ADC}$ to $\mathrm{MeV}$ conversion constant. By making this change for 200 $\mathrm{GeV}$ hadrons the mean reconstructed energy increased by approximately $4 \mathrm{GeV}$. This improvement was reflected in decreased stochastic and constant fit parameters for the energy resolution. 


\subsection{Investigation of the Topological Clustering Al- gorithm}

The previous section presented the results of the test beam data analysis using a clustering algorithm designed for the test beam environment. In ATLAS the default clustering algorithm in the FCal is topological clustering, described in Section 3.7.2. It is important to compare the results of the two techniques to both validate the topological clustering and its application to the FCal, and second, to be able to quote the FCal resolution and proposed calibrations in the context of the ATLAS experiment. The following sections explore varying the parameters of the topological clustering algorithm, in the context of electrons and hadrons in the test beam impact position 4L.

\subsubsection{Comparison of Cylinder and Topological Clustering}

The same analysis presented in Section 3.8.1 was run over the electron test beam data using the topological clustering in FCal1. The topological clustering parameters $S / N / P$ were varied to test the best configuration for electrons. Throughout this section the notation t $x y z$ denotes a topological clustering with parameters $S / N / P=$ $x / y / z$. The noise-subtracted energy resolution as a function of beam energy for five different txyz configurations is shown in Figure 3.31 (a); for comparison the cylinder clustering results labelled $\mathrm{c} 8$ are also shown with black circles. There is little difference between the cylinder clustering and the different topological clustering configurations for the highest energy electrons. As the cuts on $S / N / P$ parameters are increased the difference with respect to the cylinder clustering is increased. For all of the beam energies the worst resolution was obtained for t633, which has the most stringent cut 


\begin{tabular}{|r|c|c|}
\hline Clustering & Stochastic Term $\left[\% \cdot \mathrm{GeV}^{-\mathbf{1} / 2}\right]$ & Constant Term [\%] \\
\hline \hline $\mathrm{t} 420$ & 28.9 & 3.5 \\
$\mathrm{t} 430$ & 28.7 & 3.5 \\
$\mathrm{t} 431$ & 33.0 & 3.2 \\
$\mathrm{t} 633$ & 36.4 & 3.2 \\
$\mathrm{t} 630$ & 28.7 & 3.5 \\
$\mathrm{c} 8$ & 27.0 & 3.5 \\
\hline \hline
\end{tabular}

Table 3.11: The noise-subtracted energy resolution fit results for the stochastic and constant terms, for the default cylinder clustering (c8) and different configurations of the $S / N / P$ parameters of the topological clustering for electrons in position $4 \mathrm{~L}$.

on channels being included in the cluster.

The quantitative fit results for the stochastic and constant terms are in Table 3.11. The stochastic term is primarily influenced by the lower energy points, which do vary between configurations. The high energy beam points influence the constant term, and as a result the fitted constant term is similar for each clustering type. At low energies the individual channels have less energy deposition, so for topological clustering, which is based on the significance of the energy to the noise, there is a decreased probability of clustering channels that have lower energy deposits which have values near the cut-off for inclusion.

The electron linearity was refit for each of the clustering configurations considered. The fit results for the slope and intercept are shown in Table 3.12; the residual to these fits is shown in Figure 3.31 (b). The intercept tends to be more negative for the topological clustering than for cylindrical clustering, again relating to the fact that at low energies the topological clustering does not recover all of the energy from the low energy channels, leading to a reduced amount of clustered energy. For all beam energies the mean reconstructed energy from the topological clustering is less than cylinder clustering. 


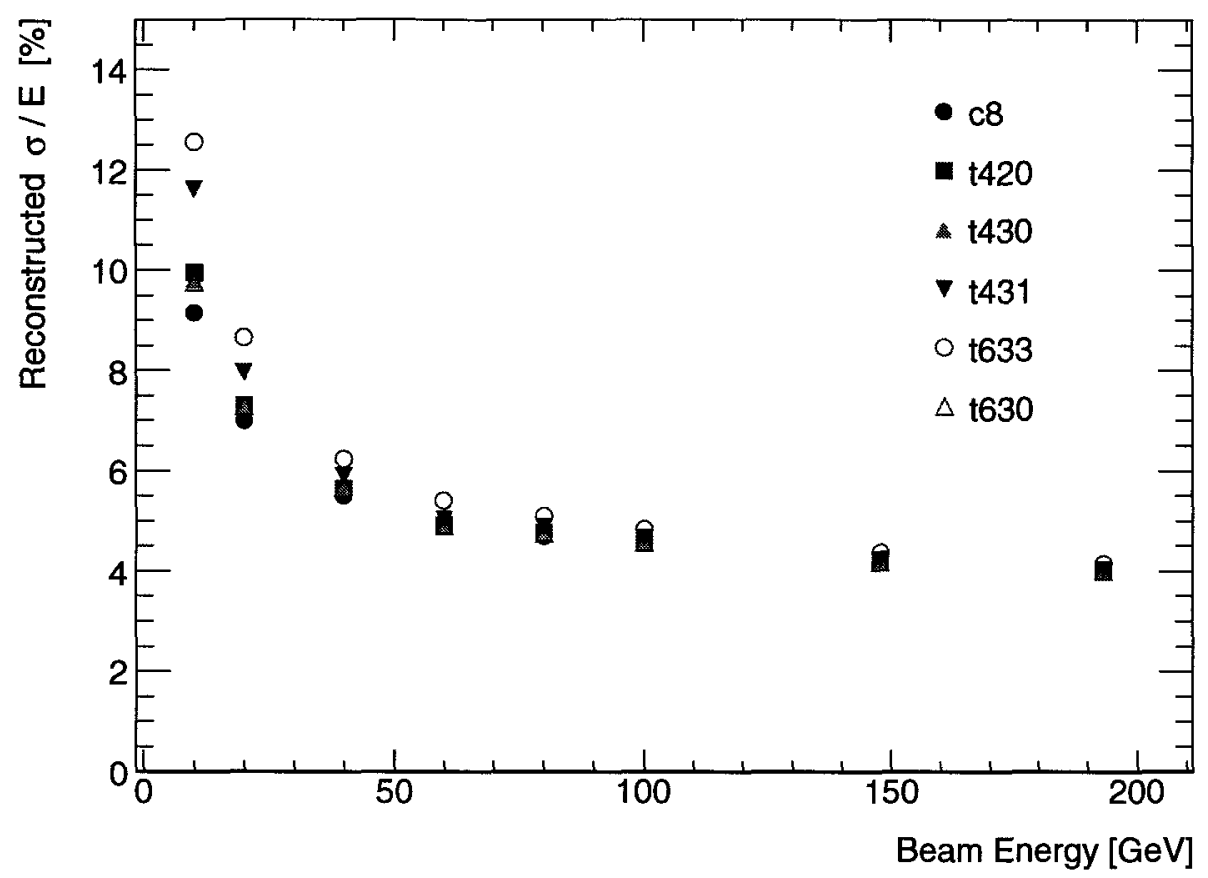

(a) The noise-subtracted energy resolution.

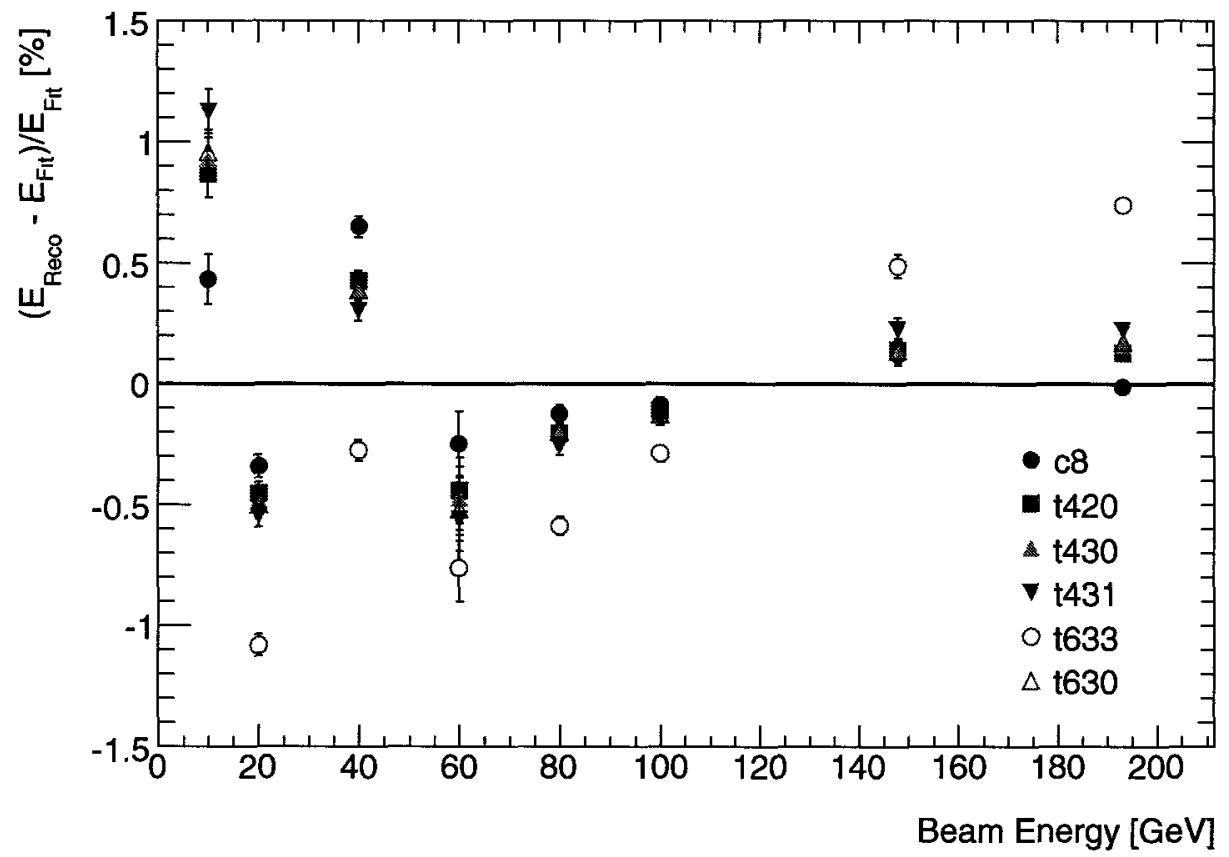

(b) The residual from the linear fit to the electron data. The $y$-axis shows the percent difference of the reconstructed energy $E_{\text {Reco }}$ from the fit energy $E_{F i t}$. The residual for the $10 \mathrm{GeV}$ t633 is off the scale at $2.56 \%$.

Figure 3.31: The (a) noise-subtracted energy resolution and (b) residual from the linear fit to the electron data for cylinder clustering and for different configurations of the $S / N / P$ parameters of the topological clustering. 


\begin{tabular}{|r|c|c|}
\hline Clustering & Slope & Intercept [GeV] \\
\hline \hline $\mathrm{t} 420$ & 1.001 & -0.96 \\
$\mathrm{t} 430$ & 0.996 & -0.99 \\
$\mathrm{t} 431$ & 0.996 & -1.12 \\
$\mathrm{t} 633$ & 0.976 & -1.96 \\
$\mathrm{t} 630$ & 0.998 & -1.00 \\
$\mathrm{c} 8$ & 0.997 & -0.77 \\
\hline
\end{tabular}

Table 3.12: Fit values for the slope and intercept for the electron linearity data clustered with five topological clustering configurations of the $S / N / P$ parameters, the results for the cylinder clustering $(\mathrm{c} 8)$ are shown for comparison.

The best configuration is one that provides the best linear response across all electron energies and minimizes the energy resolution. In ATLAS the default topological clustering for electromagnetic showers was initially t633, but has since been changed to t430. In ATLAS, the FCal is treated as a hadronic calorimeter, and the default clustering configuration for it is $\mathbf{t} 420$. There is very little difference in terms of performance for electrons between $\mathrm{t} 430$ and $\mathrm{t} 420$, and either is a good choice for topological clustering with electrons.

For electrons and photons the energy resolution in the FCal is required to be better than [26]:

$$
\frac{\sigma_{E}}{E}=\frac{35 \% \cdot \mathrm{GeV}^{1 / 2}}{\sqrt{E}} \oplus 5 \%
$$

and should be linear within $\pm 2.0 \%$. This goal is achieved for electrons in position $4 \mathrm{~L}$ for all of the tested clustering routines.

The analysis of the hadron data in position $4 \mathrm{~L}$ was analyzed in the same manner as presented in Section 3.8.2, but now entirely at the electromagnetic scale (with no flat weights applied) and with different configurations of the topological clustering $S / N / P$ parameters. A different choice of parameters were studied from those used with electrons as hadrons have wider and less dense showers and should have lower 


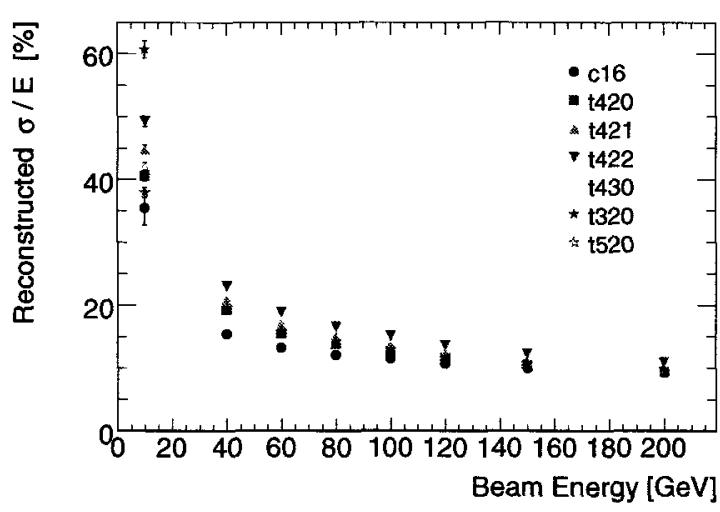

(a)

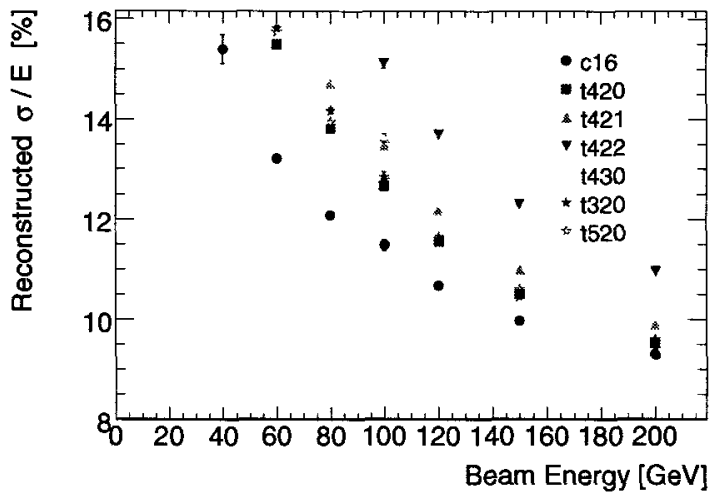

(b)

Figure 3.32: The noise-subtracted energy resolution for cylinder clustering (c16) and different configurations of topological clustering for hadrons in position $4 \mathrm{~L}$. The figure to the right shows a close up section of the higher energy beam points to emphasis the differences.

thresholds for channel clustering. The noise-subtracted energy resolutions for the various topological clustering configurations and the default cylinder clustering are shown in Figure 3.32(a), with associated fit parameters in Table 3.13. A zoom of the higher energy beam points is shown to the right in Figure 3.32(b). There is an observable difference in the resolutions for the different clustering configurations with the cylinder clustering preforming best, followed by t420, t320, and t520 which are grouped together. The linearity of the response to hadrons for each of the topological clustering cases considered is shown in Figure 3.33.

For hadrons, the energy resolution and linearity are best for the cylinder clustering. For the topological clustering cases considered here the best choices are t420, t320, and t520. This implies that the cut on the last parameter $P$ should be zero to fully cluster the energy in the shower from outlying channels that contain real but small energy depositions. To achieve the required jet energy scale resolution ATLAS requires that the energy resolution in the FCal be [30]:

$$
\frac{\sigma_{E}}{E}=\frac{100 \% \cdot \mathrm{GeV}^{1 / 2}}{\sqrt{E}} \oplus 10 \%
$$




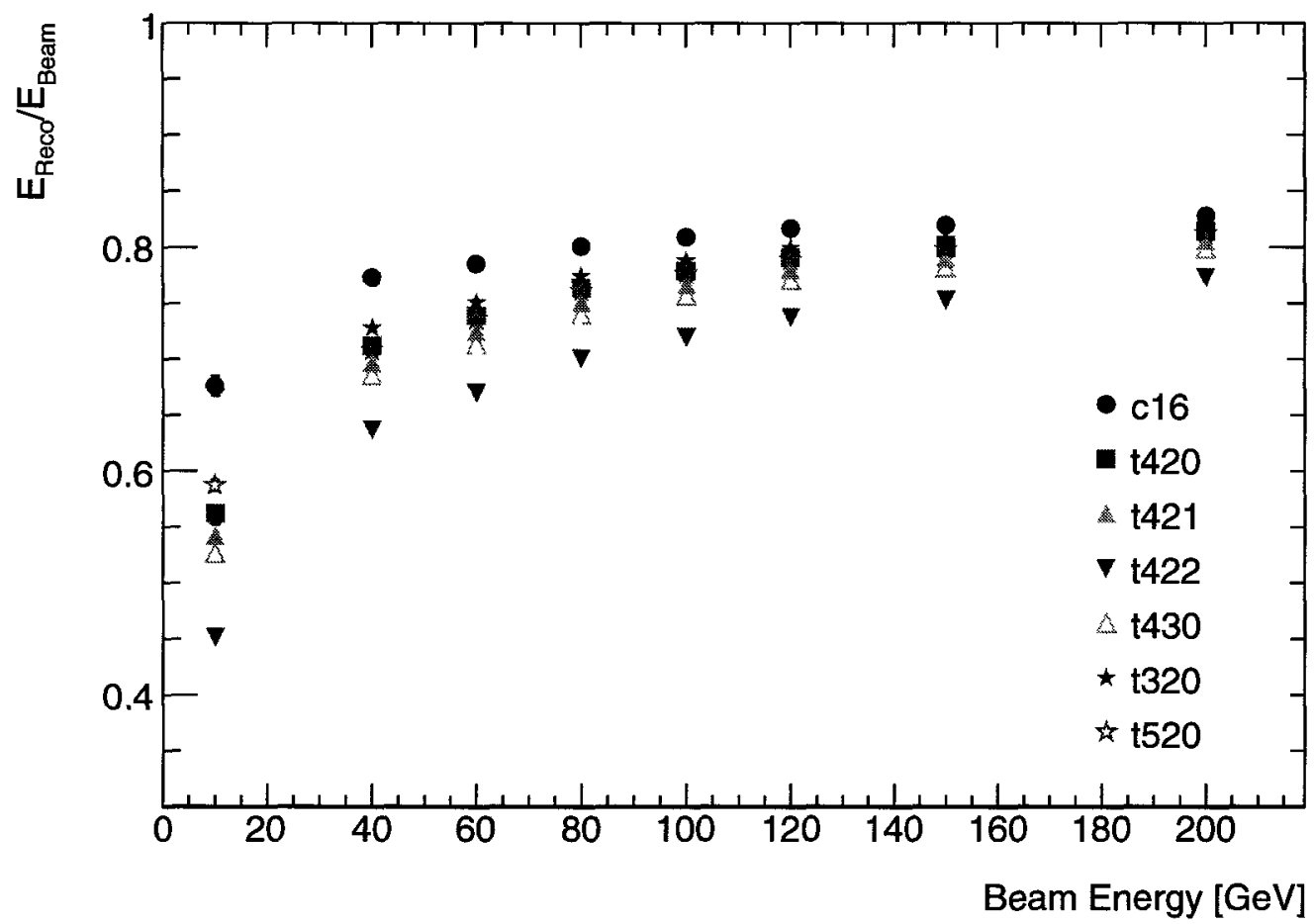

Figure 3.33: The linearity for the default cylinder clustering (c16) and six different configurations of the $S / N / P$ parameters of the topological clustering for hadrons in position $4 \mathrm{~L}$ at the electromagnetic scale. 


\begin{tabular}{|r|c|c|}
\hline Clustering & Stochastic Term $\left[\% \cdot \mathrm{GeV}^{-1 / 2}\right]$ & Constant Term [\%] \\
\hline \hline $\mathrm{t} 420$ & 114.8 & 4.9 \\
$\mathrm{t} 421$ & 125.0 & 4.3 \\
$\mathrm{t} 422$ & 143.8 & 3.9 \\
$\mathrm{t} 430$ & 126.7 & 4.6 \\
$\mathrm{t} 320$ & 119.7 & 4.0 \\
$\mathrm{t} 520$ & 116.5 & 4.8 \\
$\mathrm{c} 16$ & 88.4 & 7.0 \\
\hline \hline
\end{tabular}

Table 3.13: Noise-subtracted energy resolution fit results for the stochastic and constant terms, for the default cylinder clustering and different configurations of the $S / N / P$ parameters of the topological clustering for hadrons in position $4 \mathrm{~L}$. All values are at the electromagnetic scale.

For all of the clustering types the constant term is well below this requirement, however for the stochastic term, only the cylinder clustering meets the requirement, while the best topological clustering configurations ( $t 420, t 320, t 520)$ come close. As a reminder these results are at the electromagnetic scale. The use of a hadronic weighting scheme that corrects for fluctuations in the invisible hadronic shower component can improve these resolutions [19]. It is important to note that these results are for the impact position $4 \mathrm{~L}$, which has a minimal amount of upstream material and results in fully contained hadron showers; in moving to ATLAS there is additional upstream material in front of the FCal and leakage is possible as showers approach the inner and outer edge. The effect will be to worsen the energy resolution from the values quoted here.

Throughout the remainder of this thesis the test beam analysis will use the topological clustering algorithm with setting $S / N / P=4 / 2 / 0$ for both electrons and hadrons. 


\subsubsection{Topological Clustering Shower Shape Variables}

Once a topological cluster has been formed in ATLAS, software will be used to determine the likelihood that this cluster originated from an electromagnetic or hadronic particle. For hadrons, calibrations are applied to correct for the invisible energy losses and attempt to reduce the non-linearity of the response. Associated to the topological clustering are shower shape variables, called moments, that are used in ATLAS to help distinguish electromagnetic and hadronic showers. This section gives a brief comparison of these shower shape variables for electrons and hadrons in the position $4 \mathrm{~L}$ test beam data.

The $n$th moment of a variable $x$ for a cluster is defined as [31]:

$$
\left\langle x^{n}\right\rangle=\frac{1}{E_{\text {norm }}} \cdot \sum_{\left\{\imath \mid E_{\imath}>0\right\}} E_{\imath} x_{\imath}^{n} \quad \text { with } \quad E_{\text {norm }}=\sum_{\left\{\imath \mid E_{\imath}>0\right\}} E_{\imath},
$$

where $E_{\imath}$ is the energy deposited in the $\imath$ th channel. The sum $i$ is over all positive energy channels in that cluster. Variables of interest include those that characterize shower longitudinal depth and radial size, and shower density. The cluster center is a channel energy weighted point in 3D space $(x, y, z)$, where only positive energy channels are kept in the weighting. A cluster can also be characterized by its shower axis, the axis along which the shower is propagating (no dependence on the IP). A qualitative sketch of the shower axis $\vec{s}$ and cluster center $\vec{c}$ with respect to a channel that is a distance $\vec{x}_{\imath}$ from the IP is shown in Figure 3.34. The variables $\lambda_{\imath}$ and $r_{\imath}$ are defined below.

Figure 3.35 shows selected topological cluster moments for the highest test beam energy electron and hadron data. It is important to keep in mind that there is a small fraction of hadron and muon contamination remaining in the electron data, and muon contamination in the hadron data which can slightly alter the shapes and contribute 


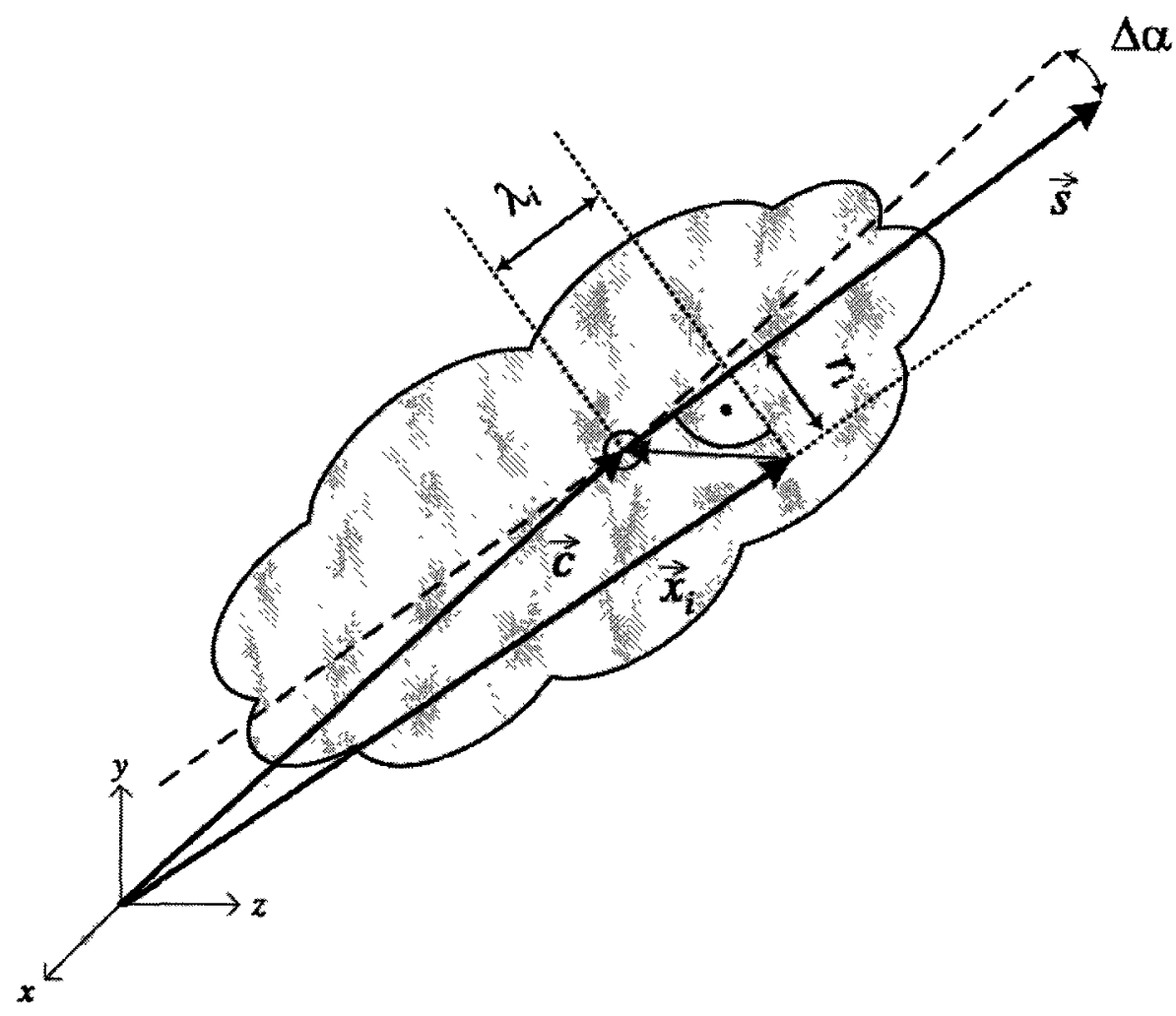

Figure 3.34: A picture of a cluster with some cloud-like shape. The shower axis is denoted by the vector $\vec{s}$ and the cluster center by the vector $\vec{c}$ which points from the cluster center to the IP. The vector for a channel $x_{i}$ is labeled as $\vec{x}_{i}$. The variables $\lambda_{i}$ and $r_{i}$ describe the longitudinal and lateral showers, and are defined in the text. Figure is taken from [32]. 
to the tails.

The first density-type moment shown is the energy density (Figure 3.35(a)), where in Equation $3.31 x^{1}=\rho=E / V$, the energy per unit volume of the channel. The second density-type moment is shown in Figure $3.35(\mathrm{~b})$, and is the fraction $\left(f_{\max }\right)$ of the total cluster energy $\left(E_{\text {total }}\right)$ in the channel with the maximum energy $\left(E_{\max }\right)$ :

$$
f_{\max }=\frac{E_{\max }}{E_{\text {total }}} .
$$

Electromagnetic showers are more dense, so as expected the average values for these moments are shifted to higher values for the electron distributions.

Hadronic showers tend to produce on average larger showers in the transverse direction. Figure 3.35(c) shows the second moment of $r$, where $r$ is the cross-product between the shower axis and channel centers, i.e. the transverse distance between channels and shower axis (shown as $\left\langle r^{2}\right\rangle$ ). The variable called 'Lateral' in Figure 3.35(d) is the normalization of the second moment of $r$ and is a complicated function defined as:

$$
\text { Lateral }=\frac{\left\langle r_{2}^{2}\right\rangle}{\left\langle r_{2}^{2}\right\rangle+\left\langle r_{\max }^{2}\right\rangle}
$$

in which

$$
\begin{aligned}
& \left.\left\langle r_{2}^{2}\right\rangle=\frac{1}{E_{\text {norm }}} \cdot \sum_{\left\{i \mid E_{\imath}>0\right\}} E_{i} r_{2, i}^{2}\right\} r_{2}=0 \text { for two most energetic channels } \\
& \left.\left\langle r_{\max }^{2}\right\rangle=\frac{1}{E_{\text {norm }}} \cdot \sum_{\left\{i \mid E_{\imath}>0\right\}} E_{i} r_{\max , i}^{2}\right\} \begin{array}{l}
r_{\max }=40 \mathrm{~mm} \text { for two most energetic channels } \\
r_{\max }=0 \text { for all other channels }
\end{array}
\end{aligned}
$$

Both lateral-type moments demonstrate that as expected the hadronic showers are on average wider in the transverse plane than electron showers. 
Hadronic showers tend to be deeper, the three longitudinal-type moments presented are described in the following sentences. The variable $\lambda$ is the dot-product of channels centers along the shower axis, and therefore is the longitudinal component of the shower (shown as $\left\langle\lambda^{2}\right\rangle$ ) in Figure 3.35(g). In Figure 3.35(e) $\lambda_{\mathrm{c}}$ is the distance from the cluster center to the calorimeter front face along a vector pointing from the cluster center to the IP (where the IP is assumed to be the ATLAS IP). The longitudinal depth of each FCal module is approximately $440 \mathrm{~mm}$. The $z$ coordinate of every channel is taken as the midpoint in the electrode. So the distance from the front face of FCal1 to each channel in FCal1, FCal2, and FCal3 is approximately $220 \mathrm{~mm}, 660 \mathrm{~mm}$, and $1100 \mathrm{~mm}$, respectively, which roughly correspond to the peaks in the $\lambda_{\mathrm{c}}$ distribution. For electrons the peak is at $220 \mathrm{~mm}$, meaning the channels clustered with significant energy are in FCal1.

The normalized 'Longitudinal' moment is similar to the Lateral moment and is defined as:

$$
\text { Longitudinal }=\frac{\left\langle\lambda_{2}^{2}\right\rangle}{\left\langle\lambda_{2}^{2}\right\rangle+\left\langle\lambda_{\max }^{2}\right\rangle},
$$

with $\left\langle\lambda_{2}^{2}\right\rangle$ has $\lambda_{i}=0$ for the two most energetic channels, $\left\langle\lambda_{\max }^{2}\right\rangle$ has $\lambda_{i}=10 \mathrm{~cm}$ for two most energetic channels and $\lambda_{i}=0$ for others in the sum over the channels in that cluster moment calculation.

The ATLAS collaboration has recently been investigating the possibility of reconstructing electrons in the forward region, including the FCal, to increase statistics for certain physics channels. The search for the decay of the $Z$ boson into two electrons $(Z \rightarrow e e)$ is one such physics channel including the FCal in the analysis. By searching for one forward $(2.5<|\eta|<4.9)$ electron in conjunction with a central electron $(|\eta|<2.5)$ the signal acceptance is expected to increase by $30 \%$ [33]. Historically, ATLAS has only been reconstructing electrons in the region of $|\eta|<2.5$, coinciding 


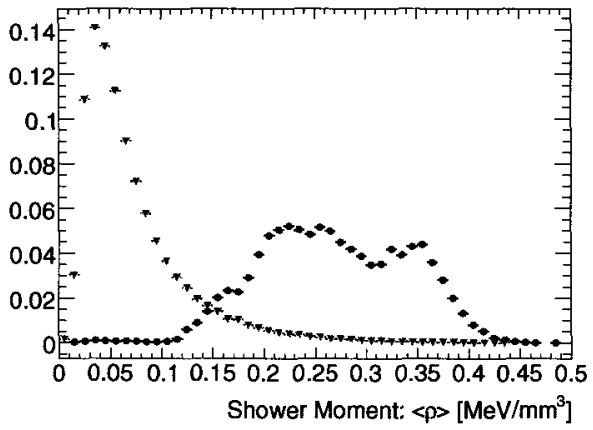

(a)

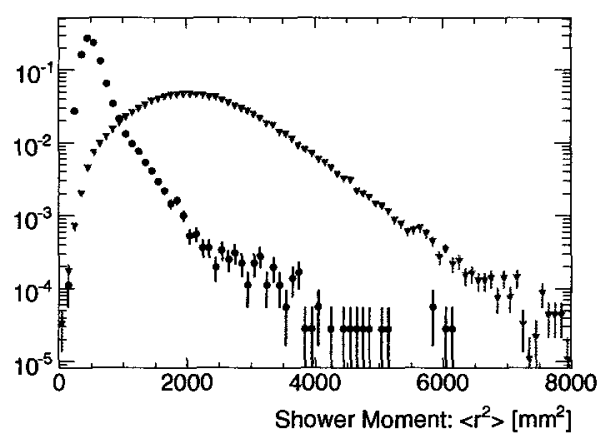

(c)

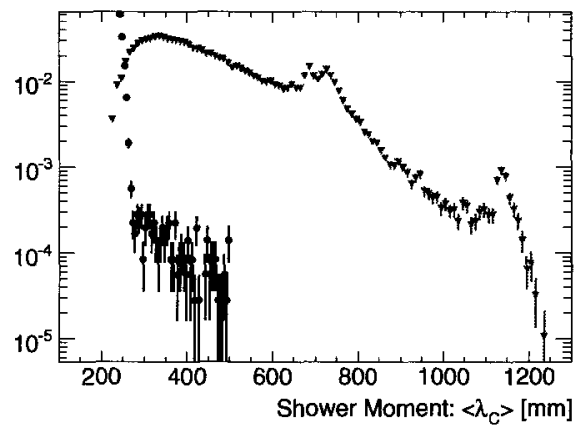

(e)

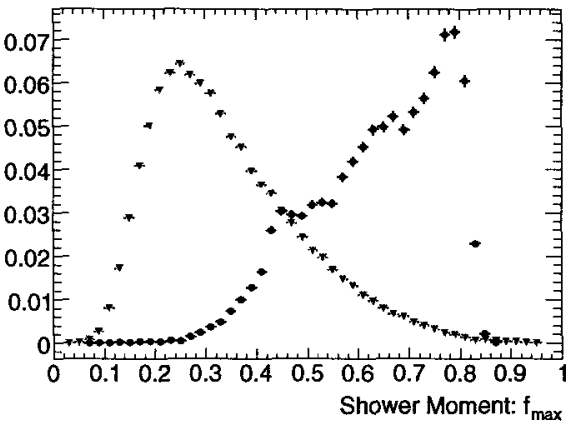

(b)

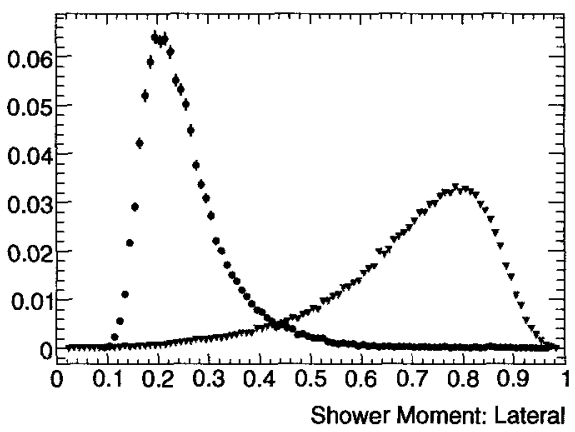

(d)

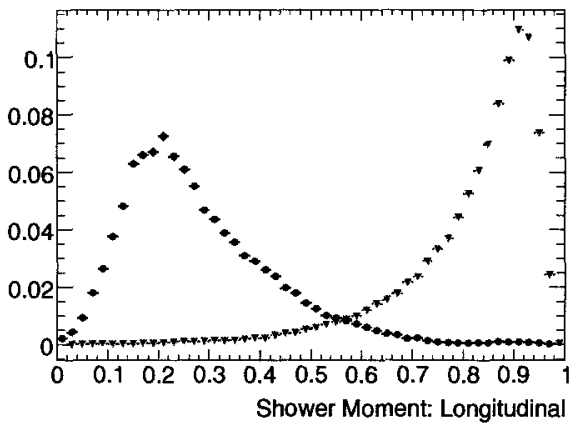

(f)

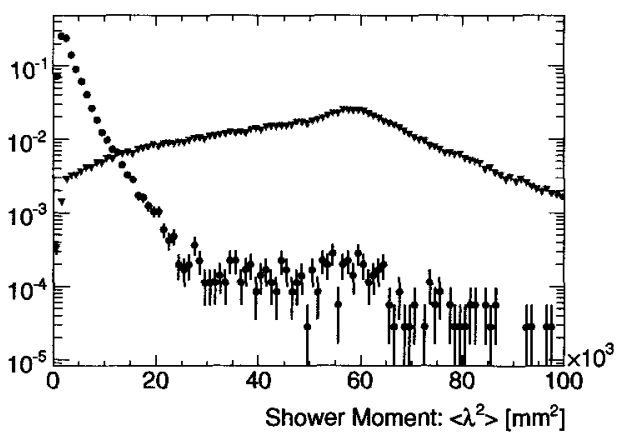

$(\mathrm{g})$

Figure 3.35: Topological clustering shower shape variables used in ATLAS to distinguish electromagnetic and hadronic showers. Shown here are the shower shape variables for the highest energy electron (black circles) and hadron (lightly shaded triangles) beam energies. 
with coverage of the inner detector tracking. In the forward regions electrons must be identified by their energy deposition in the calorimeters alone. Shower shape variables like those in Figure 3.35 are used to build a likelihood function to assign a probability for a particle to be an electron in the FCal. It is expected that the identification efficiency of electrons in the FCal will be approximately $50 \%$. The distributions shown in Figure 3.35 use real data and support the possibility that the FCal can be used to identify electrons.

\subsubsection{Topological Cluster Splitting Algorithm}

The topological cluster splitter algorithm is run by default in the ATLAS reconstruction chain [34]. It is used to split topological clusters that might have merged together from nearby objects. Hadronic showers do contain electromagnetic components and the topological splitter can be used to separate out and apply the different calibrations to them. In the splitting routine, the initial topological clusters are examined to search for regions of local maximum energy depositions that might warrant the creation of new clusters. A new local maximum is established if a channel in a cluster meets the following three requirements:

1. The channel has energy greater than $500 \mathrm{MeV}$ (called the energy cut).

2. It must have no neighbouring channels with larger energy.

3. The number of channels in the parent cluster collection must be at least four.

New clusters seeded by these local maxima are expanded by using channels already clustered using the initial topological cluster until bordering shared channels are met and split appropriately. 


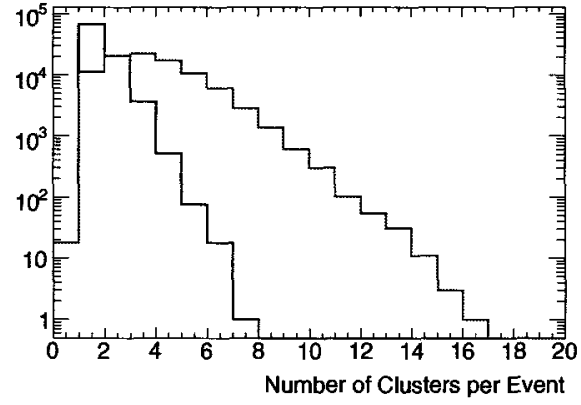

(a) Hadron data.

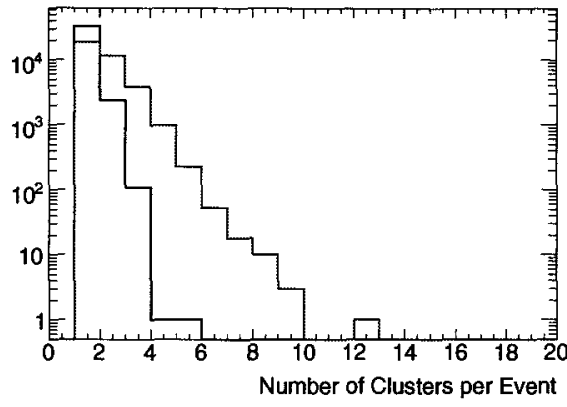

(b) Electron data.

Figure 3.36: A comparison of event cluster multiplicities when the topological cluster splitter is enabled (shaded line) and disabled (black line) to the highest energy hadron (left) and electron (right) data in position $4 \mathrm{~L}$.

In the analysis described in this section the topological cluster splitter is enabled and its effect is studied for the highest energy test beam data in position 4L. Figure 3.36(a) shows the number of clusters per event when the topological splitting algorithm is enabled (shaded line) versus disabled (solid black line) for hadron data. It is clear that the multiplicity of clusters per event significantly increases with the application of the topological cluster splitter; it is unknown whether this effect is too large, so further investigation follows.

For electrons the shower is solely due to electromagnetic components, so showers initiated by electrons should have little change in the number of clusters formed per event when the topological cluster splitter is used; the multiplicity of clusters is shown for electrons in Figure 3.36(b) when the splitter is turned off and on. The mean multiplicity increases from 1.1 to 1.7 when the splitting routine is applied to electron data. For hadrons this effect is more severe, changing the average cluster multiplicity from 1.3 to 3.4 .

To determine some of the qualities of these new clusters the geometric cluster centers in $x$ and $y$ are shown in Figures 3.37(a) - 3.37(d). For both hadrons and electrons the new clusters tend to form near the primary cluster location, where the 


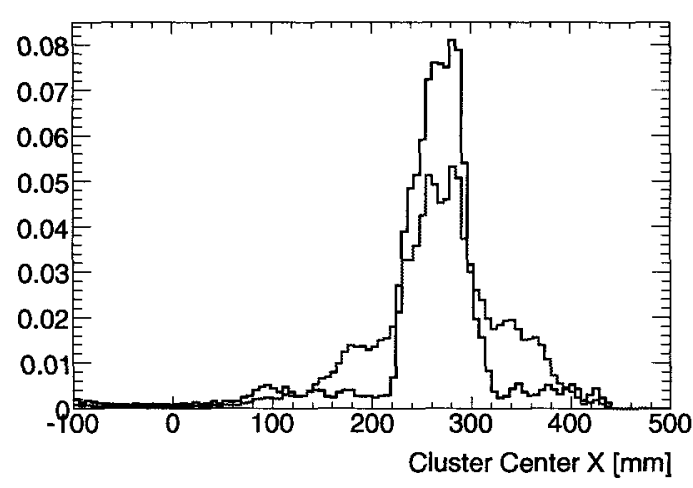

(a) Hadron data.

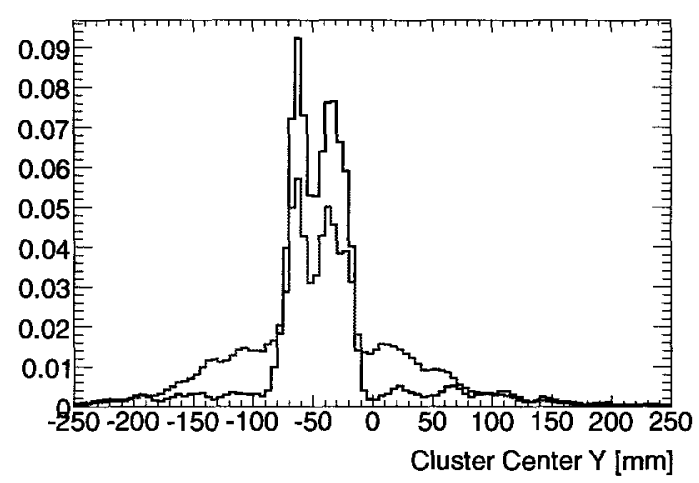

(c) Hadron data.

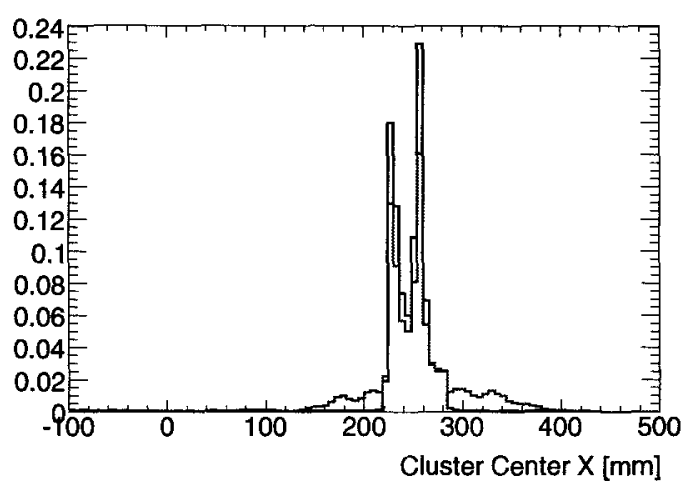

(b) Electron data.

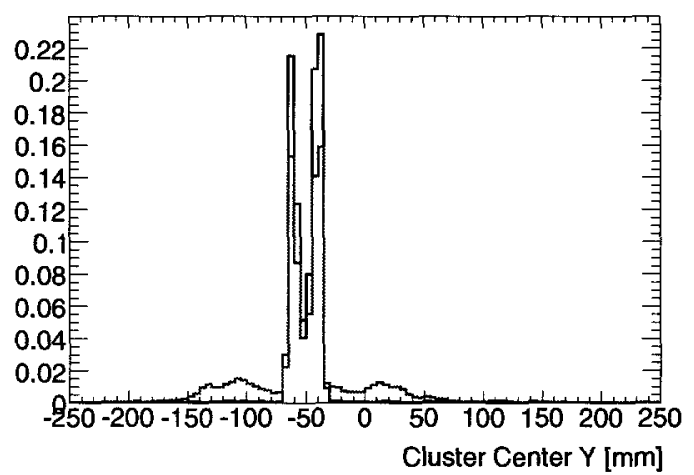

(d) Electron data.

Figure 3.37: A comparison of cluster centers in $x$ (top) and $y$ (bottom) when the topological cluster splitter is enabled (lightly shaded line) and disabled (black line) for the highest energy hadron (left) and electron (right) data in position $4 \mathrm{~L}$.

primary cluster location reflects the impact position of the incoming particle.

An interesting quantity to look at is the fraction of energy in the maximum energy cluster, shown in Figure 3.38 for electrons (right) and hadrons (left). The cluster with the maximum energy is the primary cluster, and based on the above arguments when the splitting routine is applied this primary cluster is split. For electrons the cluster energy that is lost from the maximum energy cluster is minimal. For hadrons a more interesting effect occurs, there is a tail to lower energy fractions from $100 \%$ to about $50 \%$ and then there is a steep drop off. This implies some structure to the splitting; the splitting is producing more than two clusters (as the average multiplicity almost 


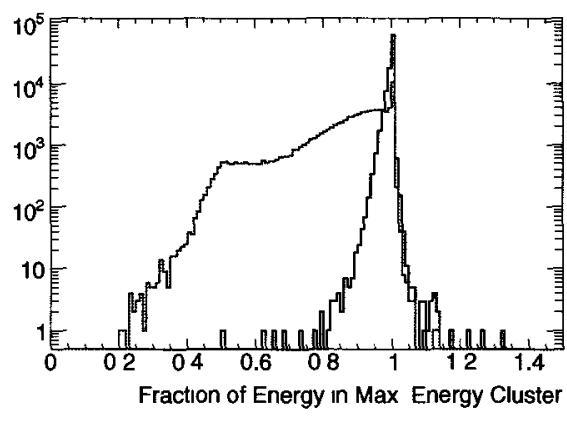

(a) Hadron data.

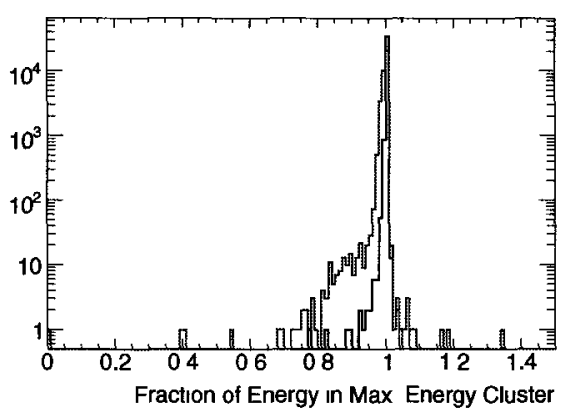

(b) Electron data.

Figure 3.38: A comparison the fraction of energy in the maximum energy cluster when the topological cluster splitter is enabled (light line) and disabled (black line) for the highest energy hadron (left) and electron (right) data in position $4 \mathrm{~L}$.

quadruples) but the primary cluster retains anywhere from $50 \%$ to $100 \%$ of the real energy, as it is depicted in Figure 3.38(a).

Variations of the default topological cluster splitter parameters were tested to investigate the significant increase in cluster multiplicity that it produced. The source of the largest variations was the energy cut requirement, which is the minimum energy for a channel to seed a local cluster maximum. In the default algorithm this energy cut is set to $500 \mathrm{MeV}$, uniformly for all ATLAS calorimeters. In FCal2 and FCal3 the noise for most channels is between about $400 \mathrm{MeV}$ and $500 \mathrm{MeV}$, whereas the expected electronic noise for the barrel calorimeters, for which this algorithm was tuned, is expected to be 10 times lower, see Figure 3.39 [7]. A random noise fluctuation in a channel in the FCal can easily fake a new local maximum.

The cluster multiplicity is quite sensitive to the choice of this energy cut parameter. Figures 3.40(a) and 3.40(b) show the cluster multiplicity for hadrons and electrons when the energy cut is set to $500 \mathrm{MeV}$ (default), $200 \mathrm{MeV}$, and $1 \mathrm{GeV}$. For reference the case where no topological splitting is used is also shown. As expected, in changing from the default energy cut $500 \mathrm{MeV}$ to $200 \mathrm{MeV}$ there is a slight increase in the number of clusters formed per event. In going from an energy cut of $500 \mathrm{MeV}$ to 1 


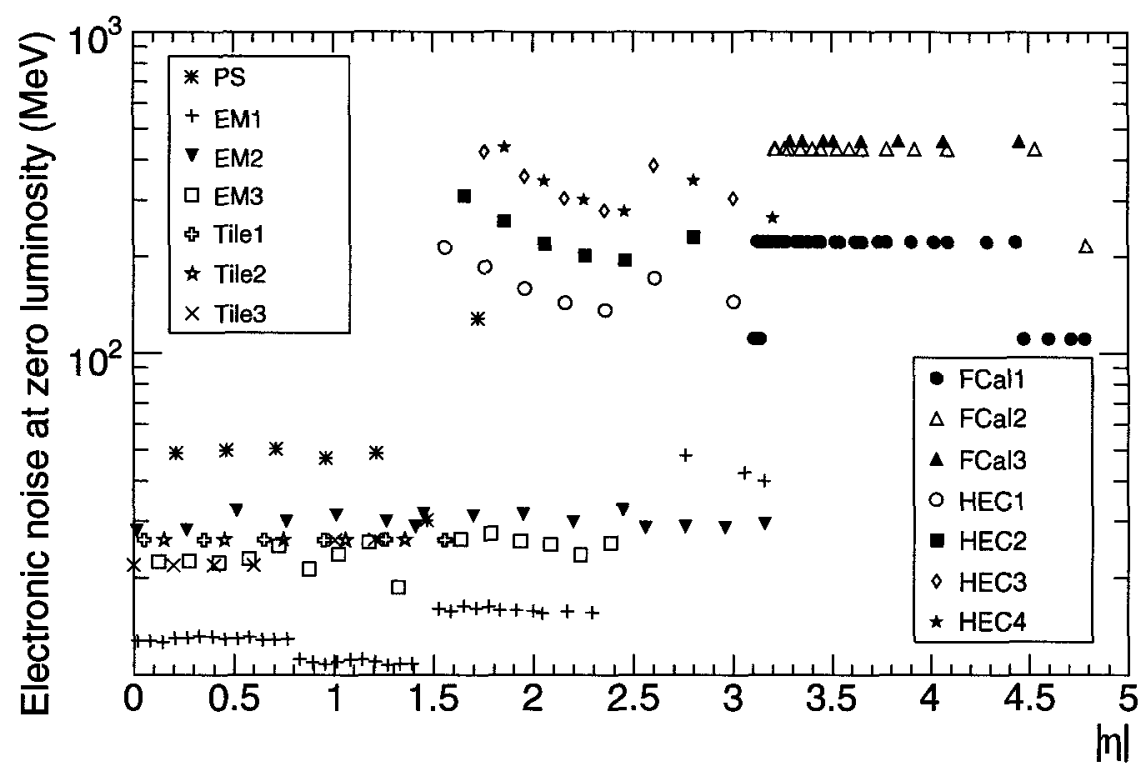

Figure 3.39: Expected electronic noise for the individual channels in the ATLAS calorimeters [7]. Notice the expected noise for the FCal is significantly greater than that in the barrel.

$\mathrm{GeV}$ there is a decreased number of clusters formed per event. For the case of the electrons the distribution using a $1 \mathrm{GeV}$ energy cut looks very similar to the case where no splitting is applied, which is the desired effect for a single electromagnetic particle entering the calorimeter.

The total reconstructed energy and resolution are unaffected by this topological cluster splitter, as long as the energy from all of the clusters are summed together, and not just the primary cluster. In ATLAS it is unlikely that all clusters in the FCal will be summed together to give the energy response to a particle, since there will be a multitude of particles from different sources and clusters seeded by pure noise. It is more likely that one or a few clusters will be summed together. The energy distribution for the cluster with the maximum energy for the case of the topological splitter off, splitter on with the default settings, and splitter on with $200 \mathrm{MeV}$ and 1 $\mathrm{GeV}$ energy cuts are shown in Figure 3.40(e) and 3.40(f) for hadrons and electrons, 


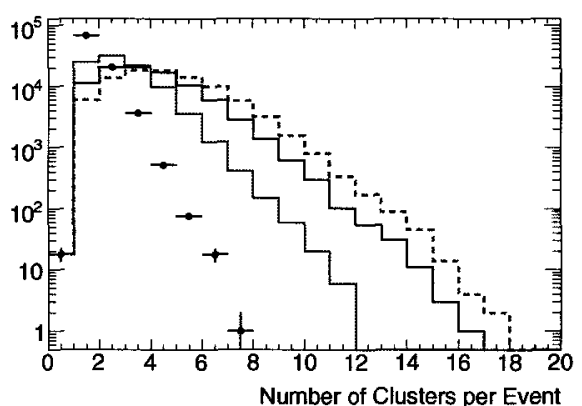

(a)

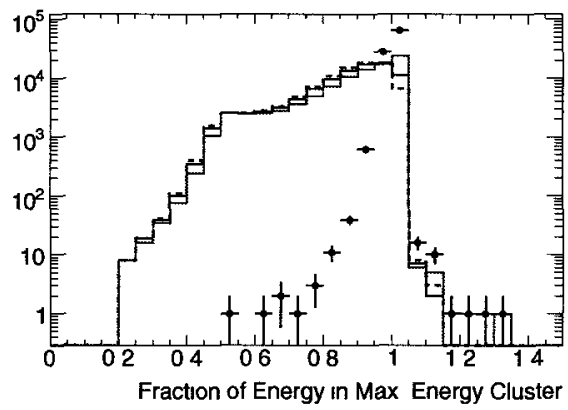

(c)

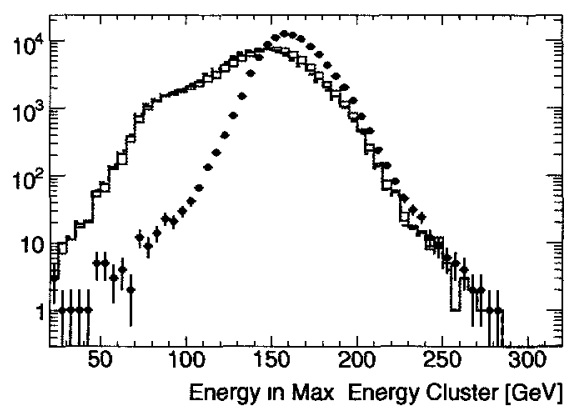

(e)

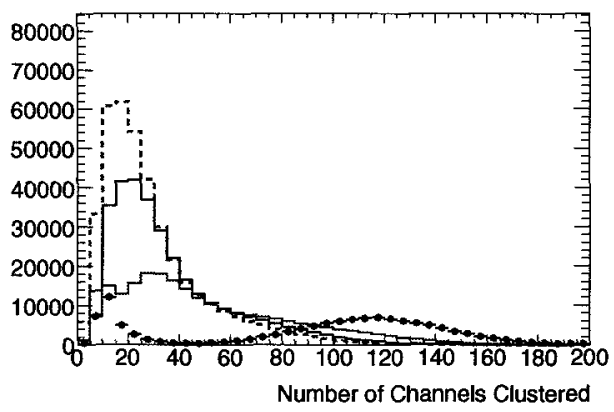

(g)

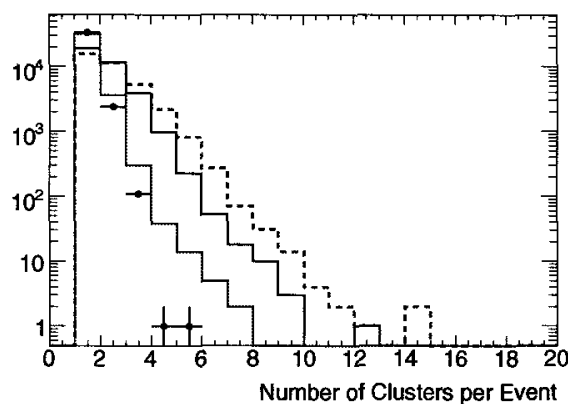

(b)

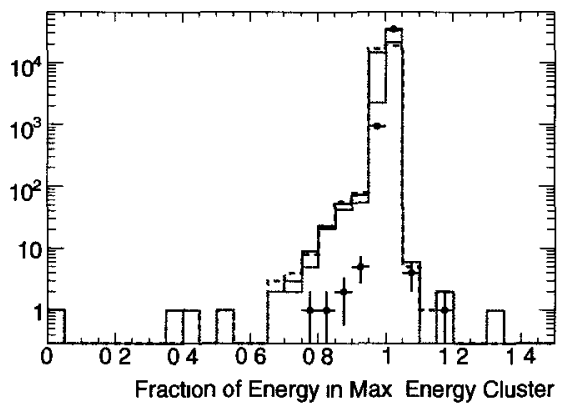

(d)

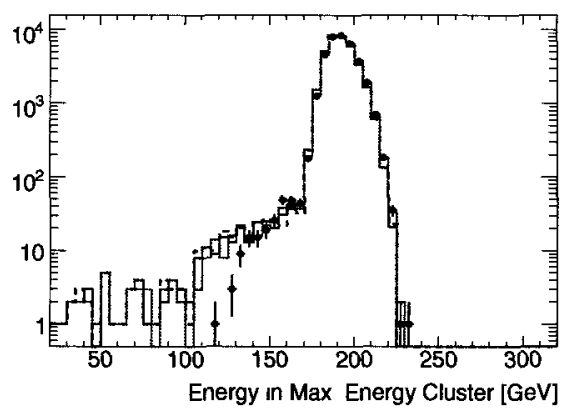

(f)

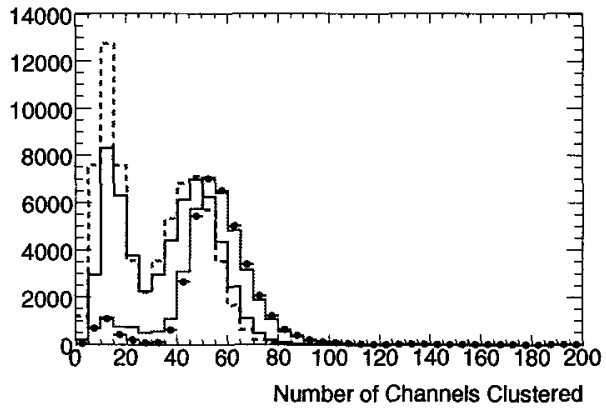

(h)

Figure 3.40: The effect of changing the topological splitter energy cut parameter from the default (black line), to $200 \mathrm{MeV}$ (shaded dashed line), to $1 \mathrm{GeV}$ (shaded solid line) as applied to the highest energy hadron (left column) and electron (right column) data. For reference the distributions when no splitting algorithm is used are shown by points. 
respectively. The shape and qualitative description of these distributions are related to the figures above showing the fraction of energy in the maximum energy cluster.

For hadrons there is a shift of the peak in the maximum energy cluster towards lower energy when the topological cluster is enabled. This could be interpreted as breaking up the primary cluster into hadronic and electromagnetic components. This splitting algorithm is normally used in conjunction with a hadronic weighting scheme that applies different weights to the electromagnetic and hadronic showers to recover the total particle energy. This topological hadronic weighting scheme is not used in the test beam analysis.

The number of channels clustered for each cluster is shown in Figures $3.40(\mathrm{~g})$ for hadrons and $3.40(\mathrm{~h})$ for electrons. Note here that the distributions are not normalized, and that the histograms representing the $200 \mathrm{MeV}$ energy cut have more entries because more clusters are produced in each event. In all cases as the energy cut is reduced the average number of channels clustered decreases, as the same number of channels are being split amongst more clusters. The peak around 10 channels per cluster represents clusters that are seeded purely by noise, and have few neighbouring channels included in the cluster. In the electron data there are approximately the same number of channels clustered for the case where the splitter is off and when it is on with a $1 \mathrm{GeV}$ energy cut. For the lower energy cuts of $500 \mathrm{MeV}$ and $200 \mathrm{MeV}$ there is a migration of the number of channels per cluster to lower values. Unlike electrons, hadrons do not have similar distributions, for the $1 \mathrm{GeV}$ energy cut and topological splitter off.

The final optimal configuration of the topological cluster splitter should be one that does not alter the number of clusters formed for electrons without its use. In this test beam analysis the final electron sample does have a very small fraction of hadron contamination, so small increases in the cluster multiplicity could be expected due to 
hadron events with electromagnetic shower components. Further studies should be made to optimize this algorithm for the FCal.

\subsubsection{Summary of Topological Clustering Results}

The topological clustering algorithm is the default clustering algorithm used by the ATLAS collaboration for the FCal. This algorithm and variations of it were applied to the test beam data to ensure the optimal choice of parameters were being used. The first investigation was of the $S / N / P$ settings. For both electrons and hadrons reconstructed in the FCal, this test beam analysis finds the choice of $S / N / P=4 / 2 / 0$ as the best configuration, which agrees with the default used in ATLAS.

Without tracking detectors in front of the FCal, particle identification must be made via characteristics of the calorimeter. Using shower shape variables computed with the clusters formed by topological clustering, it will be possible to discriminate between electrons and hadrons. The most important discriminating power comes from the properties of the channel densities, and lateral and longitudinal shower shapes.

Finally, the topological clustering splitting algorithm was studied with the test beam data. The goal of the splitter is to create separate clusters for individual particles, and to identify electromagnetic and hadronic components of the shower. In principle, applying the topological splitting algorithm to electron data should have little effect on the multiplicity of clusters created per event. The results of this analysis have found that the default topological cluster splitting algorithm is not optimized for the FCal, as there is a $55 \%$ increase in the clusters formed for electrons and a significant increase in the number of clusters formed for single hadrons. The choice of the energy cut parameter of $500 \mathrm{MeV}$, which is one quantity that determines if a new cluster should be formed, and is the same value for all of the calorimeters, is too low 
for the FCal given its higher electronic noise. The study finds an increase of the cut to $1 \mathrm{GeV}$ improves the cluster multiplicity, and yields little change in the multiplicity for electrons as compared to the case where the splitting routine is disabled. The cut of $1 \mathrm{GeV}$ has not been optimized, only shown to improve performance. A more systematic study should be conducted by scanning the effect range of energy cuts $>500 \mathrm{MeV}$.

Unless otherwise stated, the following sections of the thesis use the topological clustering algorithm $S / N / P=4 / 2 / 0$ with the topological cluster splitter turned off.

\subsection{Monte Carlo Method}

Another goal of the test beam analysis was to ensure that simulations of the FCal and physics processes occurring inside this detector were modelled correctly in the ATLAS MC software. MC simulations are routinely used to make predictions about particle production and detector reponse. These predictions are used to set limits on expected discovery and observation potentials, and so it is essential that the MC accurately models the characteristics of the data.

\section{Simulation}

The test beam used beams of known type, energy, and impact position, so beam test events can be simulated with these same beam parameters and the detector response compared to data. A MC simulation of the test beam was developed using the ATLAS Athena software framework and GEANT4 [35]. GEANT4 allows users to establish geometry volumes of materials, for which it then describes the physics processes that occur as particles travel through these materials. Particles are followed until they reach some minimum energy. That minimum energy is the energy necessary for the final particles to travel some minimum distance, called the range cut. The 
default range cut in the $\mathrm{FCal}$ is $30 \mu \mathrm{m}$.

In GEANT4, the particle interactions with matter use an assembly of models and experimental data. The combination of models are collectively called a physics list. In the ATLAS collaboration and this analysis the default GEANT4 physics list is called QGSP_BERT [36]. This physics list has been chosen as default because it has been recommended by the GEANT4 collaboration for high energy physics experiments [37], speed of computation, and it is the one that best matches the various ATLAS test beam data results.

Within a physics list there are separate models that are used for each particle type at different energy scales. Electromagnetic interactions are fairly simple and well understood, while hadronic interactions are much more complicated and therefore are much more sensitive to the choice of physics models. The following describes the models used at each energy range for high energy hadron interactions with nuclei in the QGSP_BERT physics lists.

$12 \mathrm{GeV}-100 \mathrm{TeV}$ The quark-gluon string (QGS) model is a theoretical approach to describing the result of the interaction between the incoming particle and nuclei. It is modelled by the production of one or more strings and an excited nucleus [38]. Strings connect the interacting partons in the interaction and are the basis of the fragmentation model, as the parton separate the string expands and acquires more energy. Parton pairs are produced along the string giving rise to hadronic showers. This continues until the string does not have enough energy to create new parton pairs.

$10 \mathrm{GeV}-25 \mathrm{GeV}$ For particles interacting with nuclei with energies in the range of $10 \mathrm{GeV}$ to $25 \mathrm{GeV}$ the Low Energy Parametrized (LEP) model is used to describe the fragmentation. These models parametrize experimental results for 
cross-sections, angular distributions, and multiplicities, combined with theory for conservation laws.

$100 \mathrm{MeV}-10 \mathrm{GeV}$ Intra-nuclear cascades of particles interacting with nucleons inside the nucleus are modelled using the Bertini (BERT) cascade [39]. The cross-sections and angular distributions are based on experimental data.

$<200 \mathrm{MeV}$ The de-excitation of the remaining nucleus, nucleon-nucleus interactions, and fission are described by the precompound $(\mathrm{P})$ model.

There are some regions of overlap between the models, when this occurs one model is chosen at random to decide the outcome of the interaction.

This simulation also makes use of Birk's law for recombination effects inside the liquid argon $[40,41]$. By properly including recombination effects the consequence is to reduce the overall charge collection.

The geometry of the test beam was established using volumes of specified size, location, and material reproducing the test beam environment based on the descriptions in the detailed logbook of the test beam setup. The distances along $z$ and alignment of the cryostat were such that beam particles appeared to originate from the B9 magnet. The liquid argon gaps were flagged as the active volumes, where the collection of energy measured by the detector would occur. All other parts of the FCal were designated inactive regions, and did not contribute to the energy reconstruction. Details of the test beam MC geometry and simulation calibrations can be found in [42], and were implemented primarily by J.P. Archambault (Carleton University) and $\mathrm{P}$. Thompson (University of Toronto). Note that in the simulation all of the FCal channels were read out, whereas in the real test beam only 992 channels were read out, however this should not have any impact on the analysis as no real energy was deposited in these un-instrumented regions in the data or MC. 
The original simulation included a circular beam spot with a diameter of $6.5 \mathrm{~cm}$, such that particles were equally likely to originate anywhere within that circle and propagate to the FCal. In reality the beam provided to the $\mathrm{H} 6$ line had a diameter of roughly $6.5 \mathrm{~cm}$, but often with some non-uniform distribution inside that diameter. In this analysis the real beam profile was used as input into the simulations, such that the probability of generating a particle from a particular point within the beam spot followed the same probability as that of the data. This was done to model the data conditions as closely as possible, by simulating the relative channel populations correctly, and accounting for any possible impact point dependence.

The beam population from each data run (after all event selections) were used as input for the beam profile in the simulation. For every run in the data 1,000 events were simulated in the $\mathrm{MC}$ following the beam spot distribution from that run.

For each event, the simulation returns a set of hits, which is the energy deposited in the active regions of the detector and associated time of these hits.

\section{Digitization}

After the events are simulated the energy and time of each hit in the active regions are passed along to the digitization step. The goal of this step is to convert the hits into a form similar to that of the real detector, by representing the hits as time sampled currents of the pulse shape. For each channel the following information is read from a database:

- conversion between hit energy and energy deposited in all regions of the calorimeter

(a ratio based on the amount of energy deposited in the active regions versus the total

incident energy from the simulation) 
- a conversion from energy into ADC counts (based on data results)

- pulse shape

- electronic noise

- sample autocorrelation matrix

For this test beam analysis the default values stored in the ATLAS database were used for the above parameters, and were based on results from test beam studies and early commissioning of the FCal in ATLAS. By default only five time samples were created, which differs from the real test beam environment that sampled the pulse seven times.

Following the creation of the digitized samples, the digitization step applied the OF reconstruction to the samples to extract the expected peak height, just as in the reconstruction of the real data.

The final output of the digitization stage is a reconstructed energy and time for each liquid argon channel, in a software format used by the real data.

\section{Reconstruction}

In the ATLAS MC reconstruction there are two steps. In the first step a calorimeter channel is created, holding channel identification characteristics. Once the channels are created they are supplied to the final step in the reconstruction chain, the clustering procedure. The topological clustering algorithm used in this reconstruction has identical parameters as in the data. For the MC the noise was read in from the database, using the same values as were used in the digitization.

The software used for all three steps was the same as that used in ATLAS for the official MC productions, with some small modifications where necessary to account for subtle differences in the test beam environment. 


\subsubsection{Data and Monte Carlo Comparisons}

The study of the test beam data and MC analysis focusses on comparing the properties of hadron showers as initiated by hadron data in position $4 \mathrm{~L}$ using topological clustering with configuration $4 / 2 / 0$ at the electromagnetic scale. The energy distributions for the test beam data and MC for each beam energy are shown in Figure 3.41. The high energy tail is present in the MC, which is due to the geometry of this calorimeter.

The energy distributions for the data and MC were separately fitted to a double Gaussian to parametrize the response. Figure 3.42 shows the mean reconstructed energy divided by the beam energy as a function of beam energy for the data and MC, at the electromagnetic scale. The shaded band represents the systematic error on the data, which come from examining the possible variations with respect to fitting procedure and clustering type ( $\mathrm{t} 420, \mathrm{t} 320, \mathrm{t} 520)$. The data and MC agree within $2 \%$, at the worst energy, and are within the systematic uncertainties. For all of the beam energies, the ratio of the reconstructed to beam energy is greater for the $\mathrm{MC}$ as compared to the data.

The electromagnetic response for the data and MC is close, but not exact, therefore it is insightful to compare relative quantities that are independent of the absolute scale. The fraction of the total clustered energy in FCal1, FCal2, and FCal3 is shown in Figure 3.43 for $200 \mathrm{GeV}$ and $60 \mathrm{GeV}$ hadrons. On average the $\mathrm{MC}$ hadronic showers deposit more energy in the first module. This reflects shorter longitudinal showers in the MC description of hadronic interactions with matter. On average the $60 \mathrm{GeV}$ hadrons deposit more energy in FCall than the $200 \mathrm{GeV}$ hadrons, since the longitudinal depth of a shower is correlated to the energy of the incident particle. Overall the features of the data are fairly well described by the MC. 

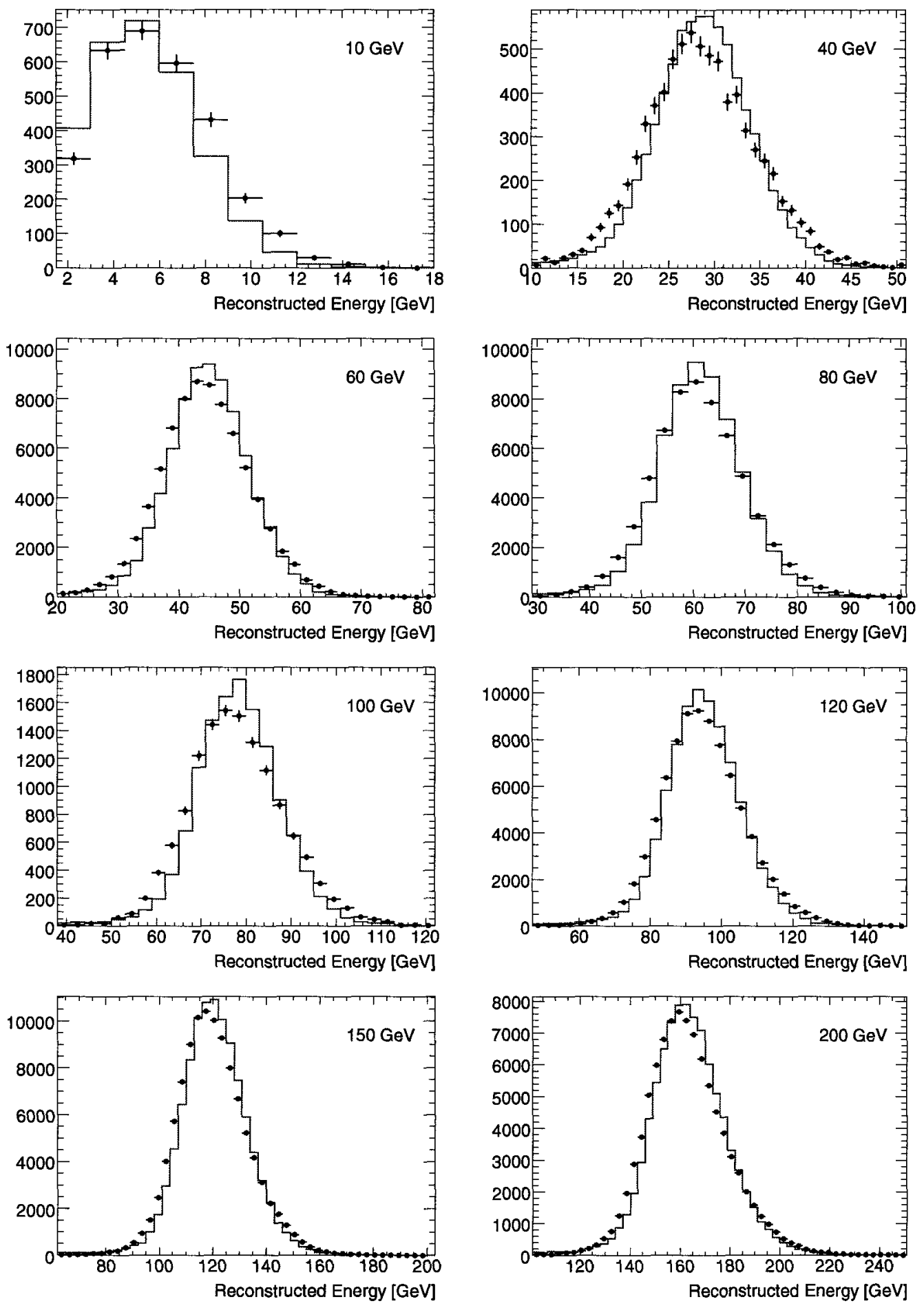

Figure 3.41: Energy distributions for hadron data (black points) and MC (histogram line) at position $4 \mathrm{~L}$. 


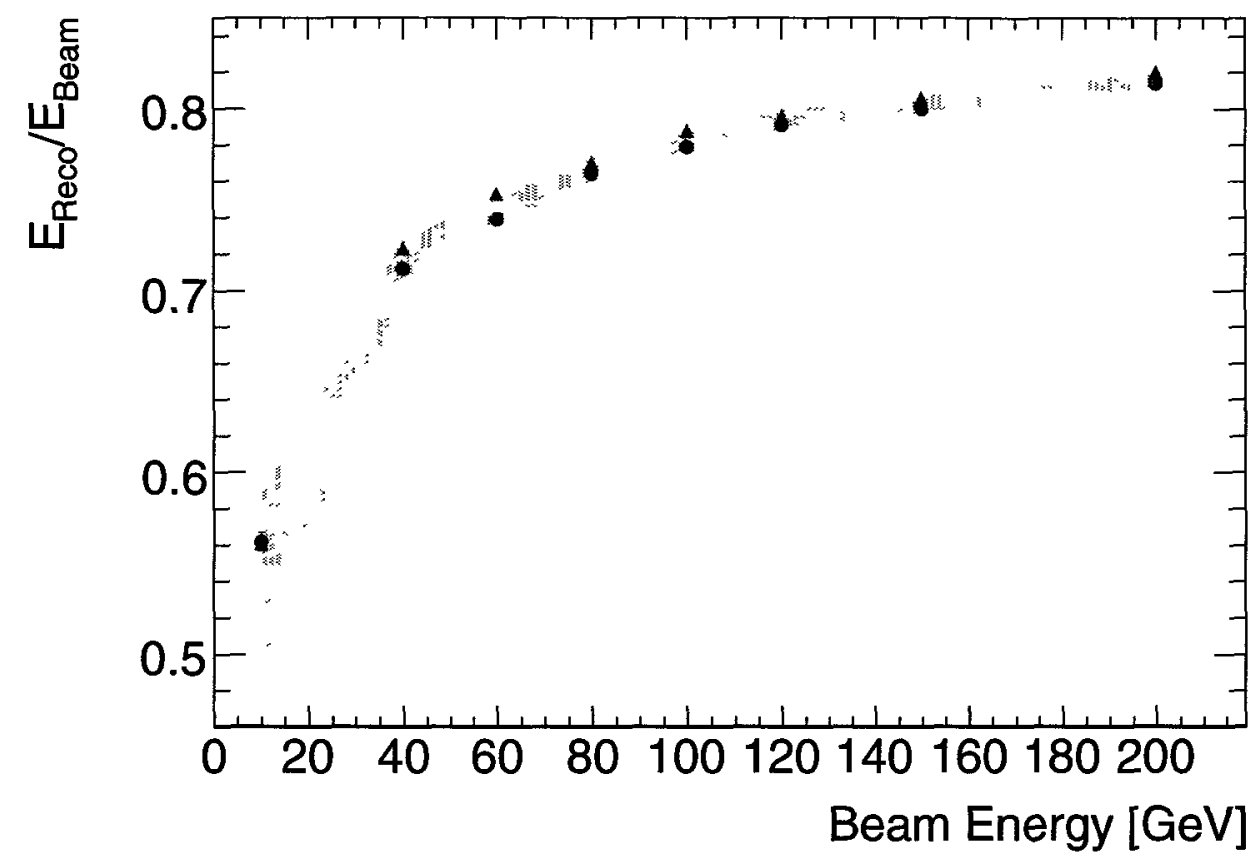

Figure 3.42: The hadron energy response for the data (points) and $\mathrm{MC}$ (triangles) at the electromagnetic scale. The shaded region represents the systematic error on the data points.

To study the transverse shower profiles the energy is summed in radial slices from the impact point of the particle, as defined using the BPC tracks for the data and true projections from the MC. A plot of the cumulative energy sum as a function of the distance from the impact point is shown in Figure 3.44 for each module normalized to the energy at a radial distance of $100 \mathrm{~mm}$. The sums include the energy from all channels in that radial distance, not just those selected by topological clustering. In FCal1 the hadron showers are narrower in the data than in the MC. In FCal1 the energy rises more quickly with radius than in FCal3, because in FCal3 the shower has a much wider geometric shape in the transverse plane. These characteristics are also tied to the longitudinal shower profiles; for the MC the showers develop much earlier, creating wider showers in FCal1.

The fraction of energy in the cluster with the maximum energy is $99.6 \%$ for both 

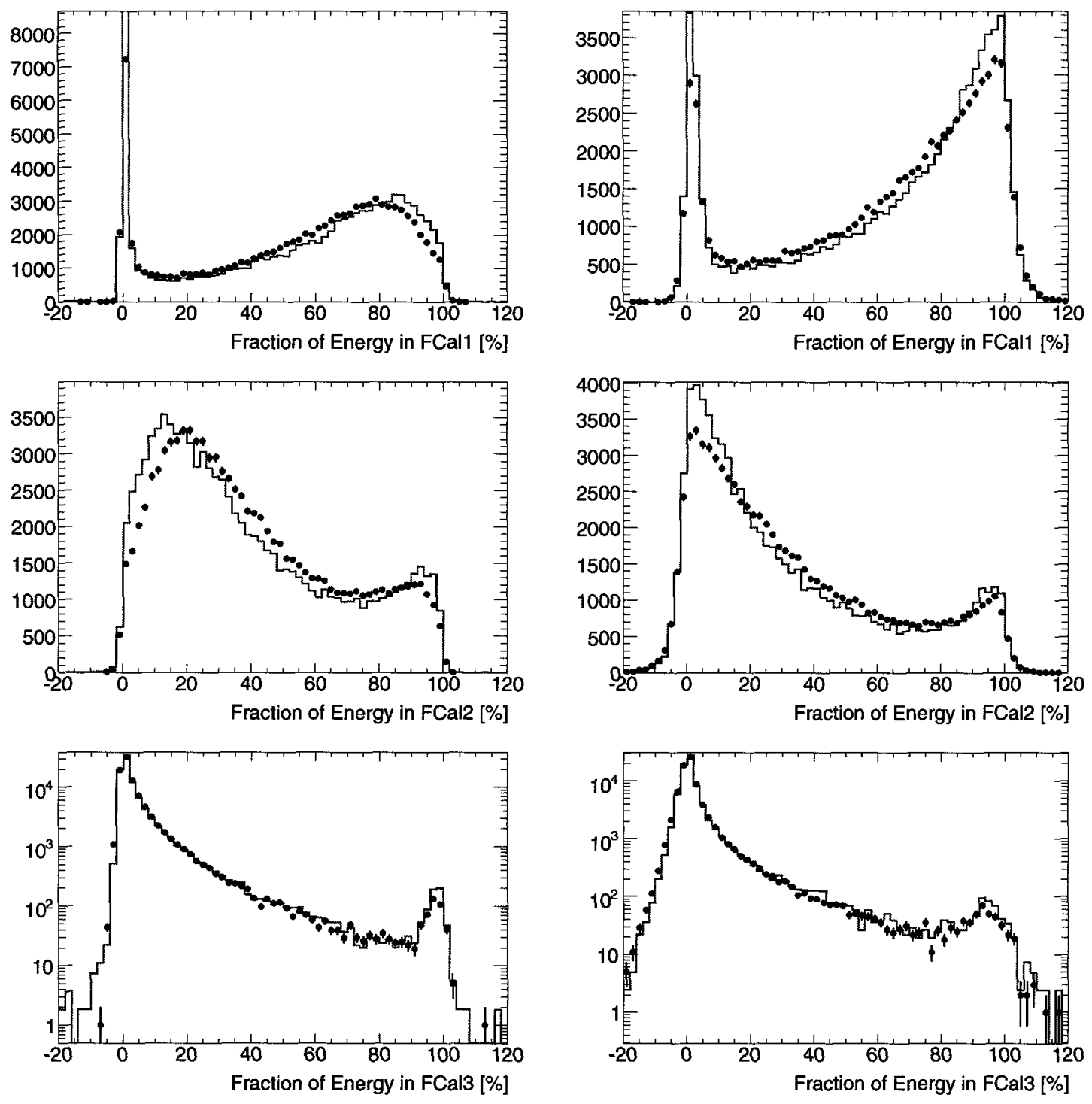

Figure 3.43: The fraction of the total energy deposited in FCal1, FCal2, and FCal3 for $200 \mathrm{GeV}$ (left column) and $60 \mathrm{GeV}$ (right column) hadron data, shown in black points, and $\mathrm{MC}$ with histogram lines. 

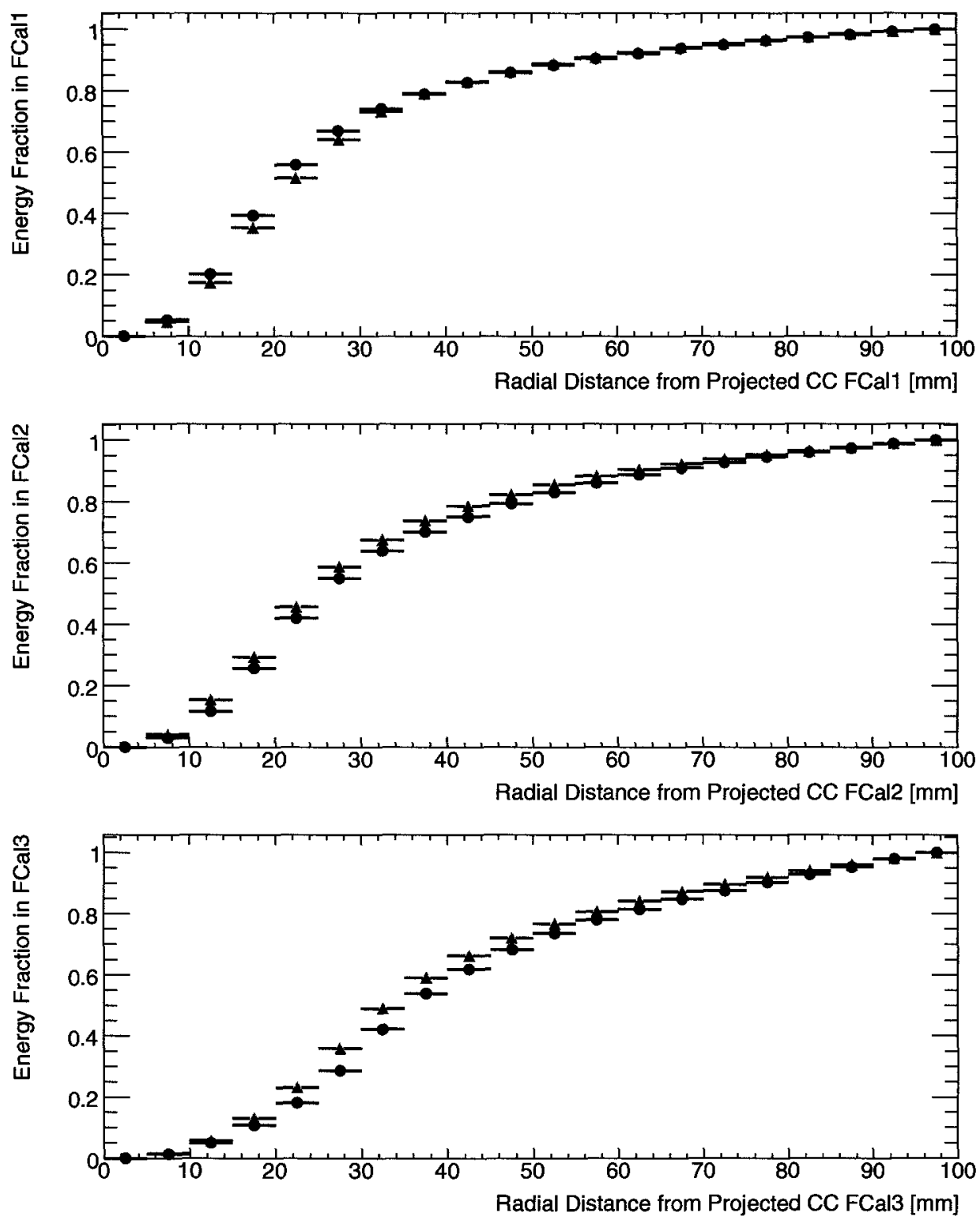

Figure 3.44: The cumulative sum of the energy as a function of radial distance from the impact position of $200 \mathrm{GeV}$ hadrons, as determined from the BPC tracking for the data and truth information for the MC. The data are shown as black dots, and the MC as triangles. 
the $200 \mathrm{GeV}$ hadron data and MC. A few cluster related quantities are discussed in the following paragraphs for this maximum energy cluster. The distribution of channel energies and the number of channels clustered in that maximum energy cluster for each module are compared for the data and MC in Figure 3.45. The data may cluster slightly more channels, but the energy of those channels clustered by the MC is greater, leading to the larger response of the MC with respect to the data. The largest difference between the data and $\mathrm{MC}$ is in the middle of the energy spectra, for low cell energies the two distributions agree well.

\subsubsection{Data and Monte Carlo Topological Cluster Shower Shapes}

In ATLAS one of the hadronic calibration schemes uses the topological cluster shower shape variables from the $\mathrm{MC}$ to identify hadronic showers to thereafter set corrections on real data. It is therefore very important to determine if these shower shapes from the MC correctly describe the shower shapes seen in the test beam data, since these calibrations will be applied to the ATLAS $p p$ collision data.

Figure 3.46 shows the distributions for the density-type shower shape variables. Figures 3.46(a) and 3.46(b) show an example of the distribution for $150 \mathrm{GeV}$ test beam data and $\mathrm{MC}$ for the cluster density $(\rho)$ and fraction of energy in the channel with the highest energy $\left(f_{\max }\right)$, respectively. Figures 3.46(c) and 3.46(d) show the mean value of the distributions for the data and $\mathrm{MC}$ across all beam energies. For comparison, the ratio of the mean value of the MC distribution divided by the mean value from the data is also shown. The density of the cluster increases with increasing energy, a reflection of the fact that the contribution from electromagnetic particles in

hadronic showers increases with energy. The MC has denser showers than the data, 

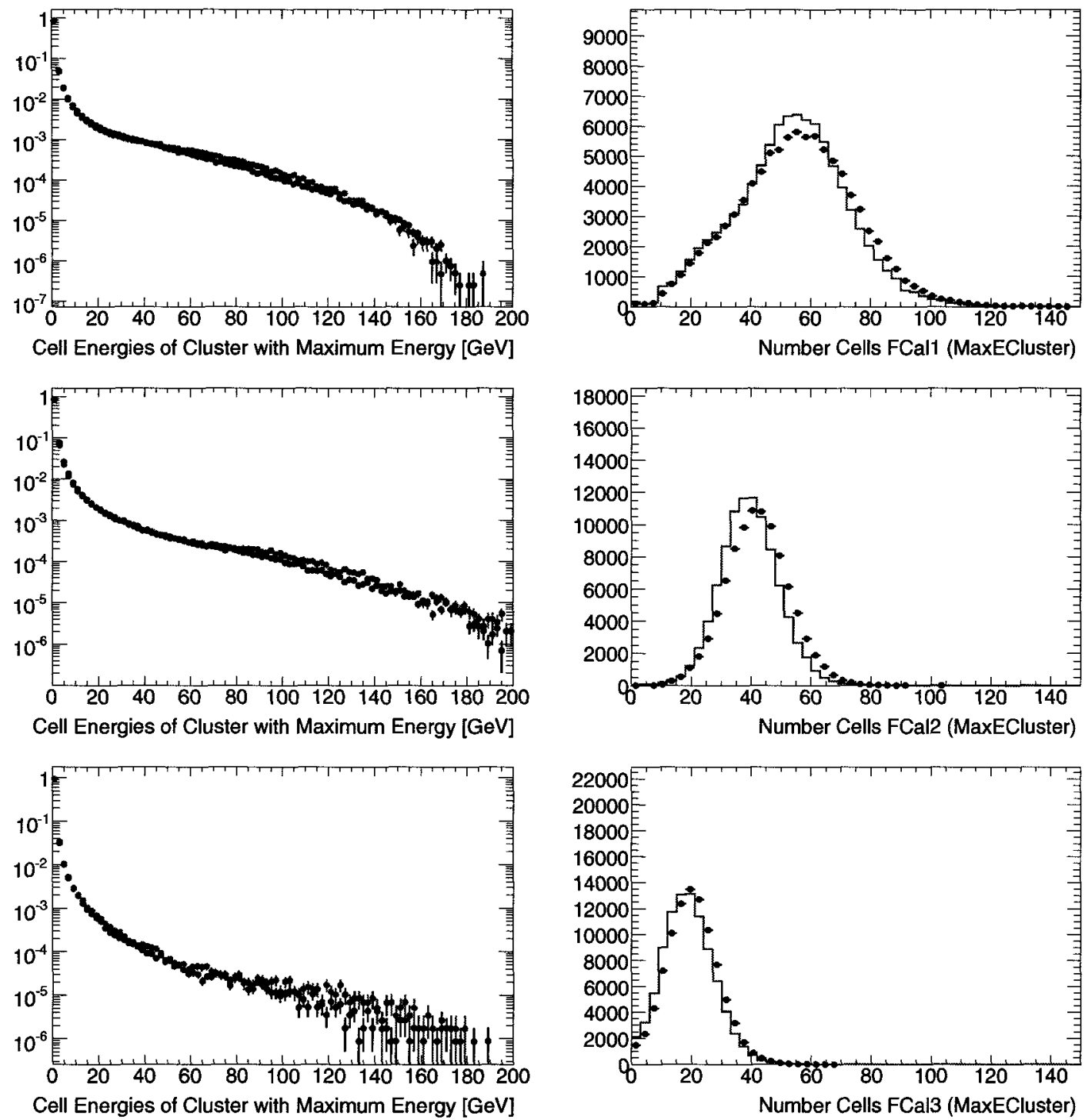

Figure 3.45: The number of channels and their energies for $200 \mathrm{GeV}$ hadron data and $\mathrm{MC}$ in FCal1, FCal2, and FCal3, for the cluster with the maximum energy in the event. The data are shown as black points, and the $\mathrm{MC}$ are in the histogram line. The term cell in the figure corresponds to channel. 


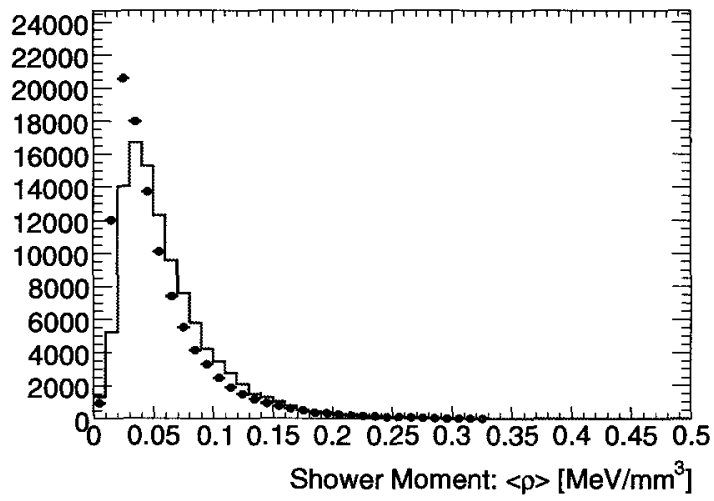

(a)

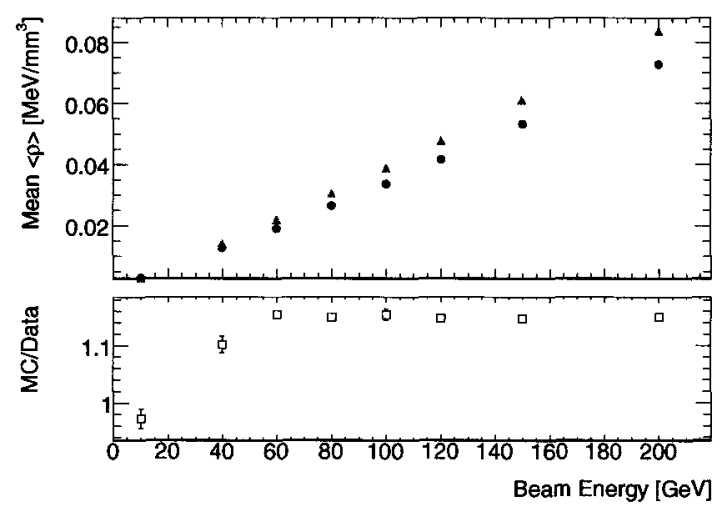

(c)

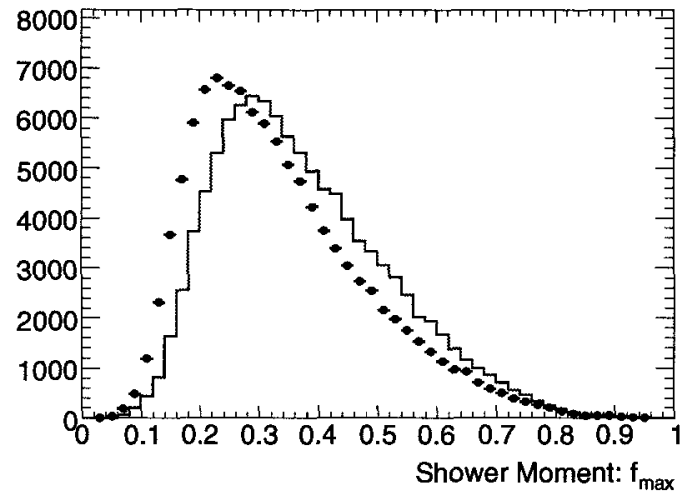

(b)

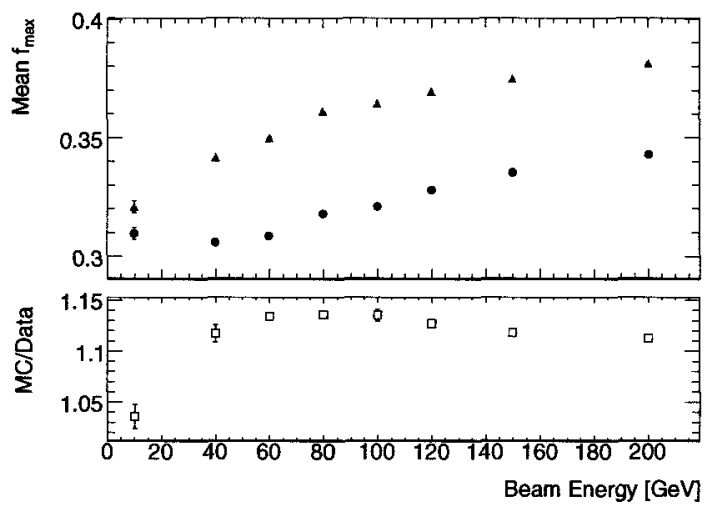

(d)

Figure 3.46: Topological clustering density shower shape variables that are used in ATLAS to distinguish electromagnetic and hadronic showers. As an example the distributions for $150 \mathrm{GeV}$ hadron test beam data (black points) and MC (histogram line) are shown in (a) and (b). Figures (c) and (d) show the mean value from each distribution as a function of beam energy for data (circles) and MC (triangles). Below, the open boxes represent the ratio of the $\mathrm{MC}$ to the data. The errors shown are statistical only.

by approximately $10 \%-16 \%$ at energies greater than $60 \mathrm{GeV}$ with a fairly constant difference. As one gets to lower energies, the ratio of the $\mathrm{MC}$ to the data tends to become closer to one.

The distributions for the topological cluster shower shape variables that describe the transverse shower shape are shown in Figure 3.47. Figures 3.47(a) and 3.47(b) show an example of the distribution for $150 \mathrm{GeV}$ test beam data and $\mathrm{MC}$ for the second moment of $r$ (the cross-product of the channels in the cluster with the shower 
axis) and the normalized lateral moment, respectively. Figures $3.47(\mathrm{c})$ and $3.47(\mathrm{~d})$ show the mean value of the distributions for the data and $\mathrm{MC}$ across all beam energies, and below the ratio of the mean value of the $\mathrm{MC}$ divided by the mean value from the data. For all beam energies the MC showers are not as wide as the data, with the $\mathrm{MC} /$ data $<1$. For energies greater than $60 \mathrm{GeV}$ the $\mathrm{MC}$ showers are about $90 \%$ the width of the data showers; with a trend towards similar widths at lower energies. The transverse shower shapes are approximately independent of the incoming particle energy above $60 \mathrm{GeV}$, which agrees with the model of particle interactions with matter presented in Section 3.1.

Figure 3.48 shows the distributions for the topological cluster shower shape variables that describe the longitudinal shower shapes. Figures 3.48(a), 3.48(c), and 3.48(e), show example distributions for $150 \mathrm{GeV}$ data and $\mathrm{MC}$ for the center of $\lambda$ moment, the second moment of $\lambda$ (recall $\lambda$ is the dot-product of the channels in the cluster with the shower axis), and the normalized longitudinal moment, respectively. Figures 3.48(b), 3.48(d) and 3.48(f) show the mean value of the distributions for the data and MC across all beam energies, and below with the ratio of the mean value of the $\mathrm{MC}$ divided by the mean value from the data. In all plots the $\mathrm{MC}$ showers are less deep than the data. For the variables $\lambda^{2}$ and the normalized longitudinal moment, the $\mathrm{MC}$ is shorter than the data by about $5 \%$ for the high energy hadrons. As has been the case with the other shower shape moments, there is a tendency for the distributions to agree better at lower energies. The un-normalized longitudinal moments are energy dependent, and represent deeper showers for higher beam energies.

The comparison of the topological shower shape variables for the data and MC indicate that the MC shower descriptions are too dense, narrow, and short as compared to the data. The response at the electromagnetic scale is greater for the MC (by $1-2 \%$ for energies greater than $40 \mathrm{GeV}$ ), possibly indicating this excess energy is 


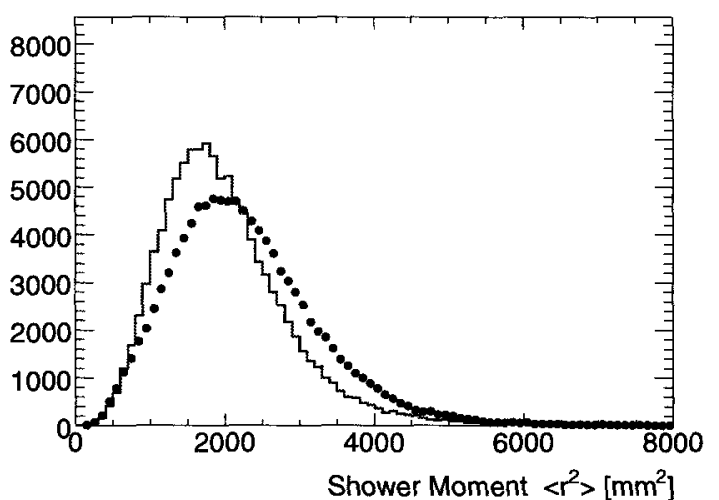

(a)

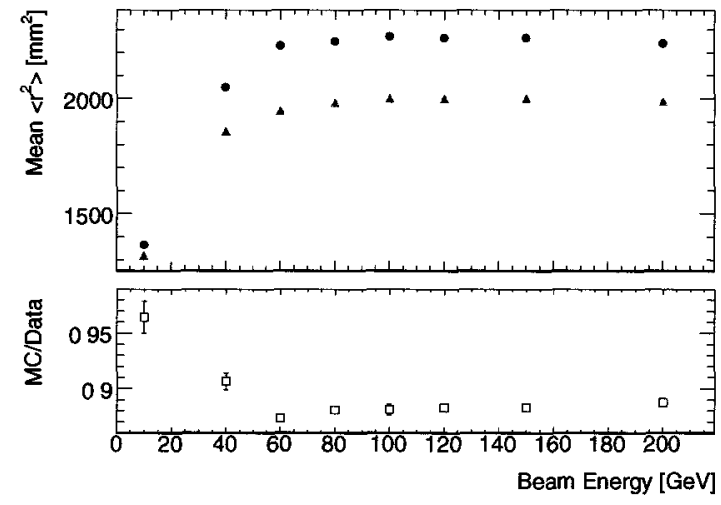

(c)

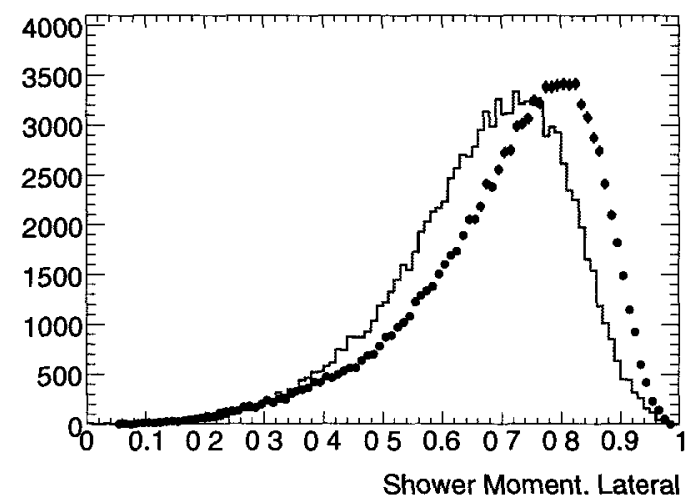

(b)

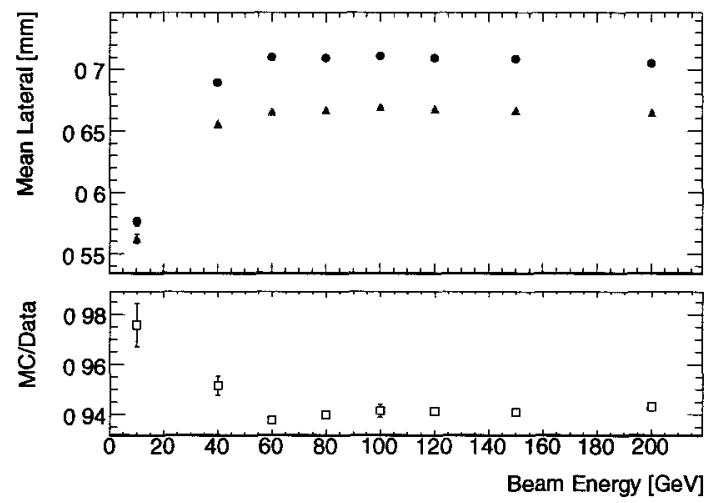

(d)

Figure 3.47: Topological clustering transverse shower shape variables that are used in ATLAS to distinguish electromagnetic and hadronic showers. As an example the distributions for $150 \mathrm{GeV}$ hadron test beam data (black points) and MC (histogram line) are shown in (a) and (b). Figures (c) and (d) show the mean value from each distribution as a function of beam energy for data (circles) and MC (triangles). Below, the open boxes represent the ratio of the MC to the data. The errors shown are statistical only. 


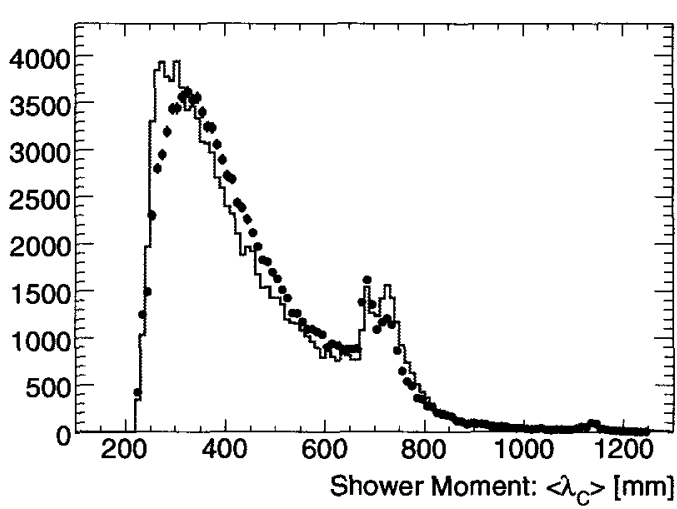

(a)

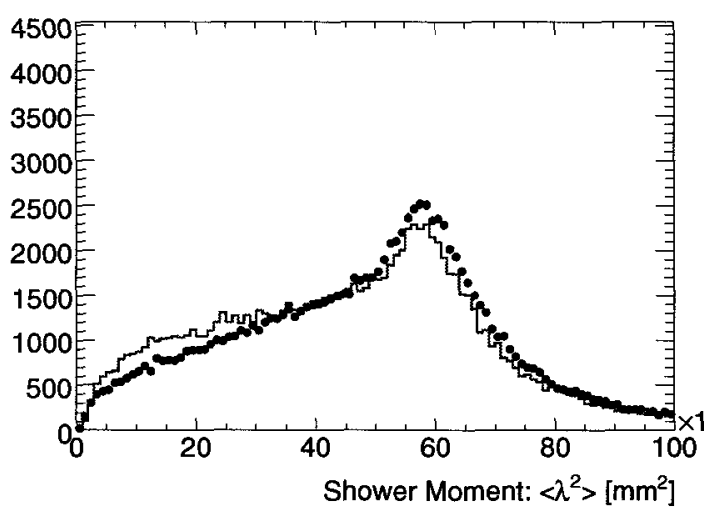

(c)

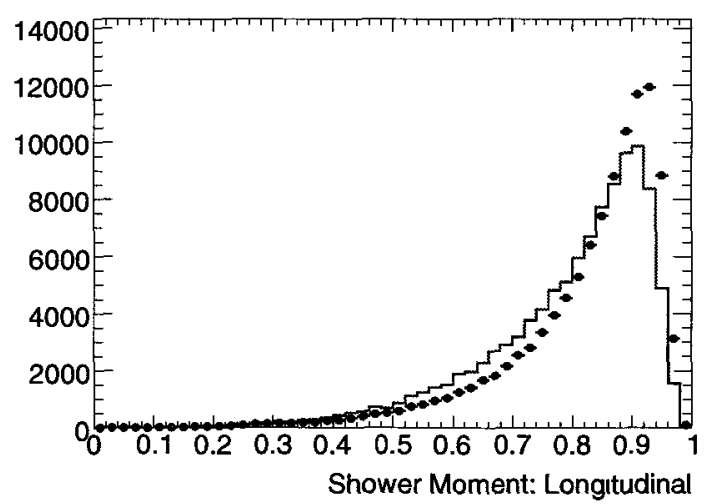

(e)

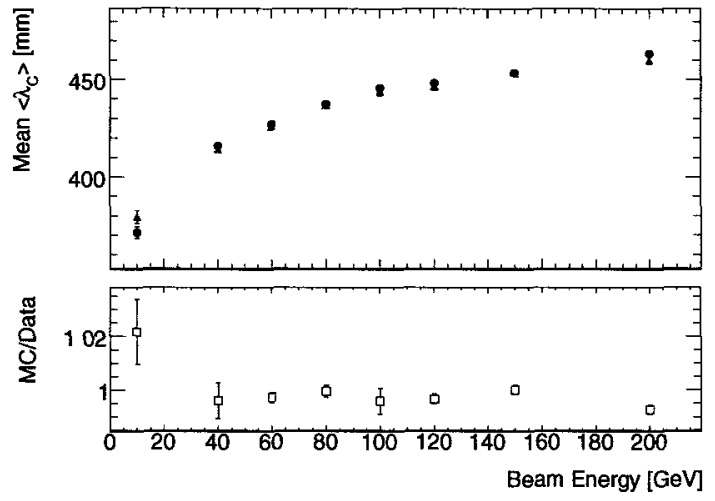

(b)

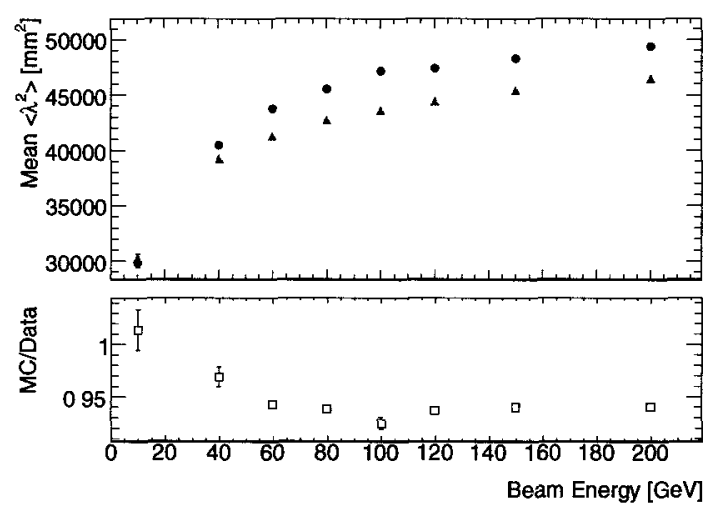

(d)

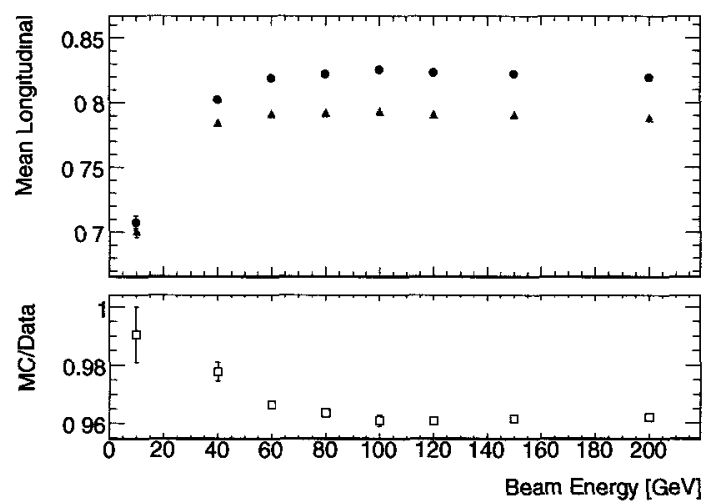

(f)

Figure 3.48: Topological clustering longitudinal shower shape variables that are used in ATLAS to distinguish electromagnetic and hadronic showers. As an example the distributions for $150 \mathrm{GeV}$ hadron test beam data (black points) and $\mathrm{MC}$ (histogram line) are shown in (a), (c), and (e). Figures (b), (d), and (f) show the mean value from each distribution as a function of beam energy for data (circles) and $\mathrm{MC}$ (triangles). Below, the open boxes represent the ratio of the $\mathrm{MC}$ to the data. The errors shown are statistical only. 
being deposited in a narrow cone around the incoming particle near the front of the calorimeter. Other ATLAS test beam studies of the barrel calorimeter also find that the GEANT QGSP_BERT physics list produces particle showers that are more narrow than seen in data [32]. Alternative physics lists are being explored in ATLAS for use in future simulations. One that shows promise is the FTF_BERT physics list which changes the model of the interactions at high energies $(>1 \mathrm{GeV})[32]$.

\subsection{Response of the Forward Calorimeter at High $|\eta|$}

The ATLAS calorimeter system is almost perfectly hermetic, with a few cracks between different calorimeter structures. One of the largest regions without calorimeter coverage is beyond the inner radius of the FCal, where the LHC beam pipe enters ATLAS. The FCal provides calorimeter coverage up to an $|\eta|$ of 4.9. As a particle enters the calorimeter at an impact point close to the inner edge there is an increased chance that some of its energy will not be contained in the FCal, leaking out of the detector along the beam pipe. It is necessary to understand how showers develop near this inner edge region, and understand the energy loss associated with the showers produced by these particles at very high $|\eta|$.

The study of the response of the FCal at high $|\eta|$ has been done with $200 \mathrm{GeV}$ hadron data at test beam impact positions 1,2 , and 3 . As a reminder, these points span a range of approximately $4.3<|\eta|<4.9$. The clustering used is the topological clustering algorithm with the default ATLAS settings previously described, and with the topological splitting algorithm disabled. The analysis was done at the electromagnetic scale. The total number of events after all cuts for each impact position are 


\begin{tabular}{|c|c|c|c|}
\hline & Position 1 & Position 2 & Position 3 \\
\hline \hline Number of Events & 209319 & 120467 & 181738 \\
\hline
\end{tabular}

Table 3.14: The total number of events after all cuts for the hadron data used for the inner edge analysis.

in Table 3.14.

An example of the location versus clustered energy deposition is shown in Figure 3.49 for one $200 \mathrm{GeV}$ hadron event at impact position 1 . The beam pipe is shown as the empty circle in the center. The size of the squares represent the read out channel size. Some channels at the inner and outer edge are smaller, formed by one electrode group. As can be seen from this figure there are energy deposits all around the beam pipe, despite the fact that the particle strikes FCal1 on the right side. The showering of the energy around the beam pipe propogates through all of the modules. In the topological clustering algorithm new clusters across the beam line are formed that are not adjacent to any channels from the primary cluster.

The total clustered energy per event (summed over all clusters) for the $200 \mathrm{GeV}$ hadron data at each impact position is shown in Figure 3.50. For reference the $200 \mathrm{GeV}$ hadron data at position $4 \mathrm{~L}$ is also shown. The beam particles at impact position 3 hit the FCall front face between $100 \mathrm{~mm}$ and $170 \mathrm{~mm}$ from the center of the beam line. As the inner edge of the FCall is at $72.3 \mathrm{~mm}$ it is possible that many of the hadrons showers for position 3 were not fully contained in the calorimeter. There was also additional upstream material in front of the FCal at position 3 compared to position $4 \mathrm{~L}, 5 \mathrm{~cm}$ of aluminum and $10 \mathrm{~cm}$ of polyethylene (roughly $0.25 \lambda$ ). This results in some additional energy loss upstream of the FCal. These differences cause a decrease in the mean reconstructed energy and wider variation in the response at position 3 compared to position $4 \mathrm{~L}$. 

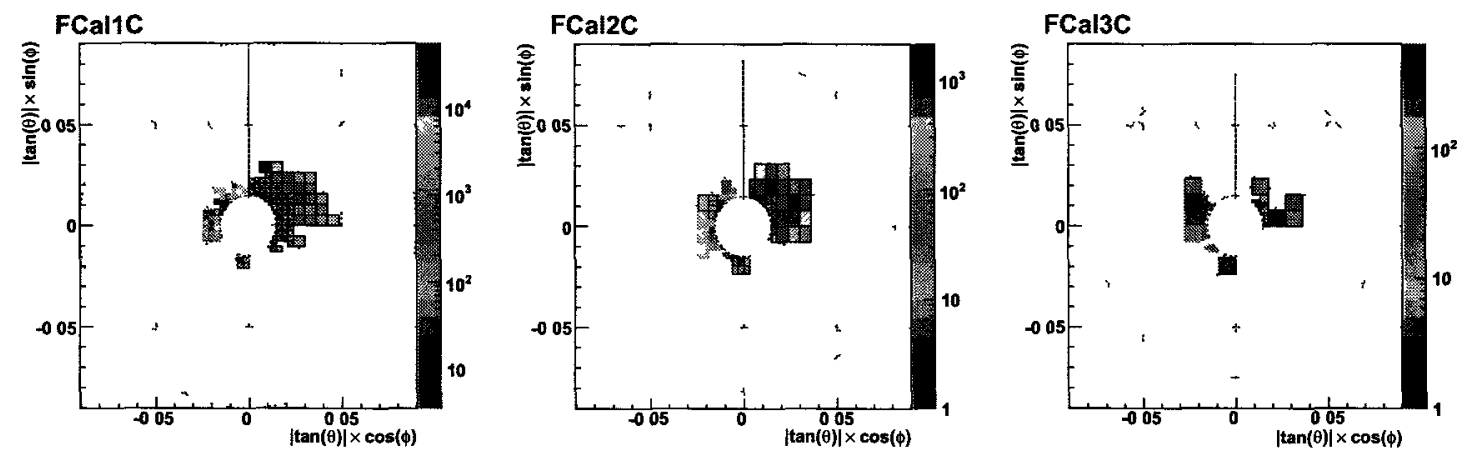

Figure 3.49: Event display of channel positions and energies for channels clustered in one $200 \mathrm{GeV}$ hadron at impact position 1 . The test beam used the $\mathrm{C}$-side modules of the FCal.

The energy spectrum for position 2 has a low energy shoulder at approximately $70 \mathrm{GeV}$. This is an artifact of the uneven population of the beam spot and variation of the response with $R$. This was checked with energy distributions from MC simulations using the same beam particle profile as in the data. A MC with a uniform beam distribution had a smooth low energy tail. The width of the energy distribution in position 2 is significantly greater than that of position 3 , reflecting the energy losses and shower variability associated with particles striking the FCal at increasing $|\eta|$.

For impact position 1, the energy distribution (Figure 3.50(d)) shows a wide main peak at around $140 \mathrm{GeV}$, and then a long low energy tail, caused by the presence of the beam line and the splashing of energy across it.

\begin{tabular}{|l|c|c|c|c|}
\hline & Position 1 & Position 2 & Position 3 & Position 4L \\
\hline \hline Frac Energy FCal1 [\%] & $47.9 \pm 31.3$ & $57.8 \pm 29.2$ & $58.8 \pm 29.2$ & $56.4 \pm 29.5$ \\
Frac Energy FCal2 [\%] & $39.1 \pm 24.9$ & $38.5 \pm 26.4$ & $37.9 \pm 26.3$ & $40.0 \pm 26.5$ \\
Frac Energy FCal3 [\%] & $17.9 \pm 25.4$ & $6.8 \pm 12.2$ & $6.4 \pm 12.0$ & $6.7 \pm 12.3$ \\
Average Number of Clusters/Evt & $2.38 \pm 1.50$ & $1.99 \pm 1.18$ & $1.59 \pm 0.87$ & $1.32 \pm 0.59$ \\
Frac in Max. Energy Cluster [\%] & $93.4 \pm 9.5$ & $97.9 \pm 3.8$ & $99.3 \pm 1.7$ & $99.6 \pm 1.1$ \\
\hline
\end{tabular}

Table 3.15: The fraction of the total clustered energy in FCal1, FCal2, and FCal3. Also shown is the cluster multiplicity and fraction of energy in the maximum energy cluster for the four impact positions. The errors quoted are statistical uncertanties only. The distributions themselves typically do not follow a Gaussian distribution. 


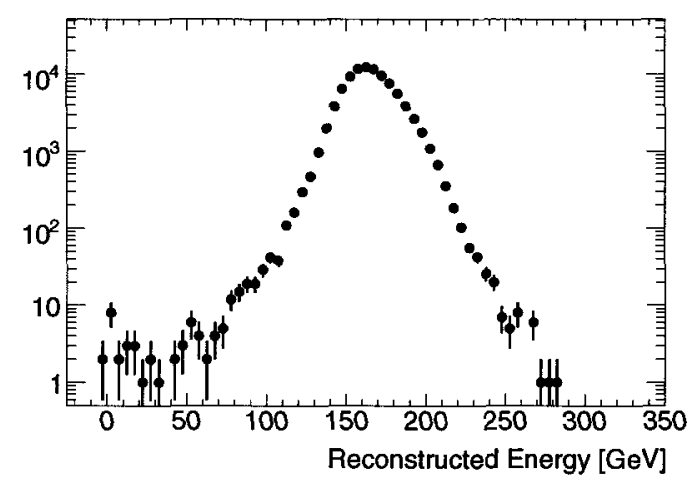

(a) Position $4 \mathrm{~L}$

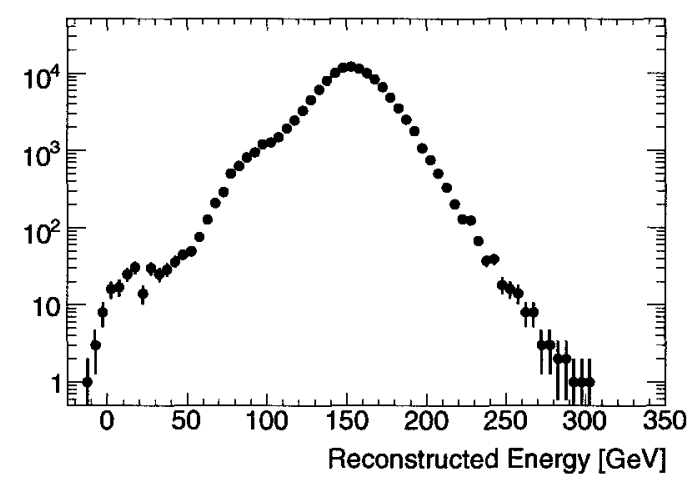

(c) Position 2

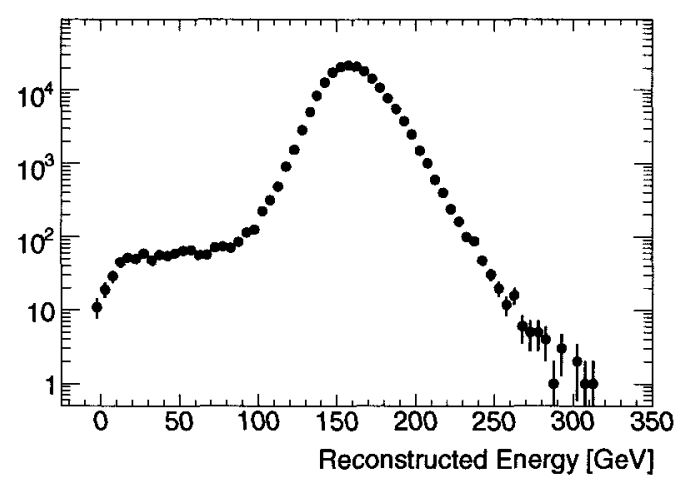

(b) Position 3

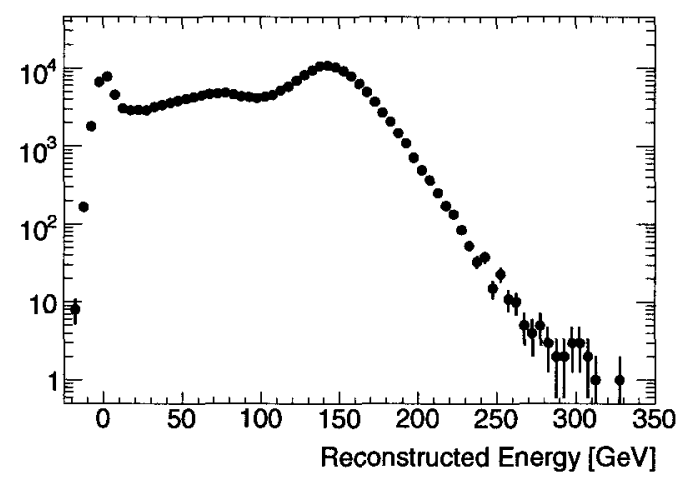

(d) Position 1

Figure 3.50: The total clustered energy at the electromagnetic scale (FCal1+FCal2+FCal3) for $200 \mathrm{GeV}$ hadrons at the five impact positions. 
The properties of the energy deposition can be compared for each position. Along the longitudinal direction the fraction of energy deposited in FCal1, FCal2, and FCal3 is compared; the first three rows of Table 3.15 show this mean value for each module. With the additional material upstream for impact position 3 the showers tend to start earlier than for position 4L, depositing more energy on average in FCal1. Position 1 had slightly more upstream material than the other inner edge positions, $3.0 \mathrm{~cm}$ thick aluminum, which should have caused the showers to start even earlier. One would expect the fraction of the total energy deposited in FCal1 to be higher in position 1 than for the other positions. However, from Table 3.15 one can see the fraction of energy in FCal1 for position 1 is in fact the lowest. Impact position 1 was positioned such that particles would strike on average between $40 \mathrm{~mm}$ and $110 \mathrm{~mm}$ from the center of the beam line, so half of the particles go through the hole in the center of FCal1 $(r<72.3 \mathrm{~mm})$. Although the showers could start earlier with the added upstream material, the particles that do not first interact with the FCal at the front face of FCal1 continue traversing the beam line and can interact with materials of the beam line and cryostat eventually producing showers that deposit more energy in FCal3.

Also shown in Table 3.15 is the average number of clusters per event, and the fraction of energy in the cluster with the maximum energy. Position 1 tends to form a higher multiplicity of clusters, with clusters forming across the beam line that carry some small fraction of the total reconstructed energy. For position $4 \mathrm{~L}$ there tends to be 1.32 clusters per event, with the main cluster containing almost $100 \%$ of the event's energy; other clusters are primarily formed by noise fluctuations. As one gets closer to the inner edge more clusters are formed per event with the consequence of splitting the shower energy among more clusters.

In ATLAS the reconstruction of an object like a candidate electron or hadron is 
unlikely to merge clusters that form on different sides of the beam pipe. This, tied with the leakage, will result in a decrease of the total reconstructed energy of an object which can have adverse effects when calculating particle energies or missing energy. The test beam data is used to propose a cluster-based correction to account for the energy loss. In an attempt to recreate the way objects are reconstructed in ATLAS, only the cluster containing the maximum energy (93.4\%-99.3\% of the total clustered energy) is considered in the proposed correction.

The mean reconstructed energy as a function of the radial distance from the center of the beam line is shown in Figure 3.51. The $x$-axis shows the radial distance $R$ from the center of the beam line using the BPC track projections in $x$ and $y$ to the front face of FCal1, $R=\sqrt{x^{2}+y^{2}}$. The value $R=0$ corresponds to the center of the beam line. The average reconstructed energy (in the cluster with the maximum energy) is shown on the $y$-axis. The inner edge of FCal1 is at $R=72.3 \mathrm{~mm}$. Even below this radius there is some energy deposition because the particle can interact with the material of the beam pipe, and/or graze the FCal beyond its front face (the particle enters with some small angle, 0.88 degrees).

The data in Figure 3.51 represent the data from impact positions 1, 2, 3, and 4L. Impact positions 1,2 , and 3 span a range from $R=40 \mathrm{~mm}$ to $R=170 \mathrm{~mm}$. There is a gap for the region between $R=170 \mathrm{~mm}$ and $R=220 \mathrm{~mm}$ where no test beam data were available (refer back to the test beam impact positions in Figure 3.7). The data from impact position $4 \mathrm{~L}$ span a range of $R=220 \mathrm{~mm}$ to $R=290 \mathrm{~mm}$. The mean reconstructed energy as a function of radius for position $4 \mathrm{~L}$ is flat, reflecting fully contained showers at this sweet spot in the FCal.

There is a steep rise of the average reconstructed energy from about $20 \mathrm{GeV}$ to $160 \mathrm{GeV}$ between $50 \mathrm{~mm}$ and $90 \mathrm{~mm}$, then the slope slowly increases toward a plateau region, which is the average response of the FCal to hadrons at the electromagnetic 


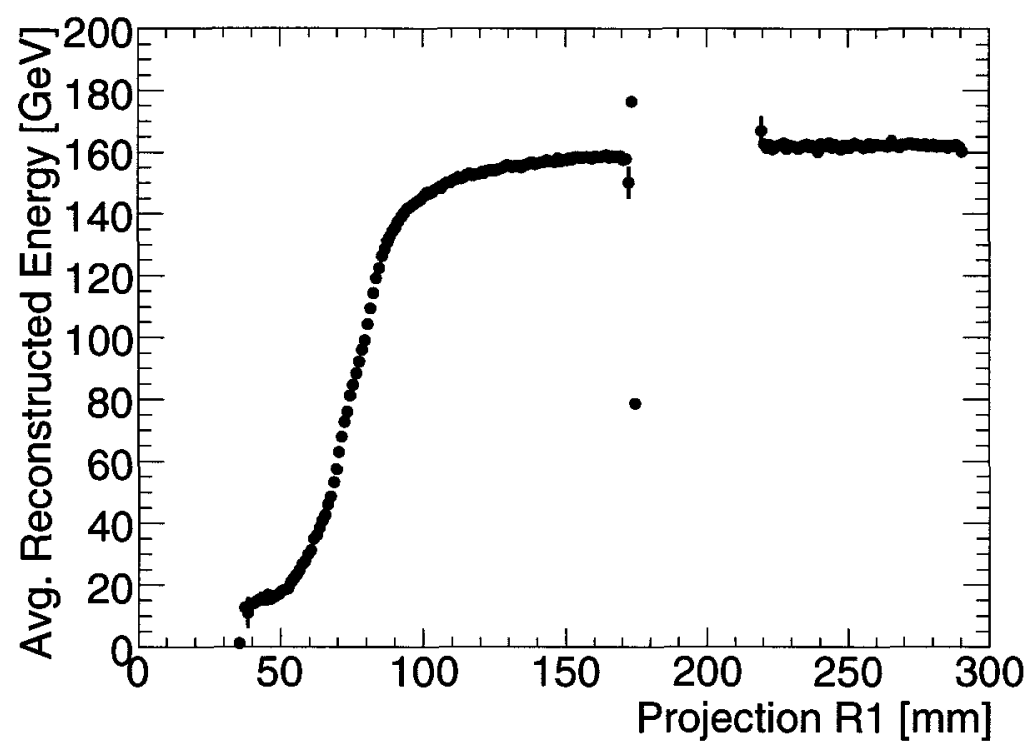

Figure 3.51: The mean reconstructed energy as a function of radial distance from the center of the beam line for $200 \mathrm{GeV}$ hadrons using the data from impact positions 1,2 , 3 , and $4 \mathrm{~L}$. The radial distance Projection $\mathrm{R} 1$ is calculated by projecting the reconstructed tracks from the BPCs onto the front face of FCal1.

scale for showers that are fully contained. There is a slightly reduced response for position 3 (100 $\mathrm{mm}$ to $170 \mathrm{~mm}$ ) with respect to position $4 \mathrm{~L}$, due to the added upstream material.

Figure 3.51 uses tracking information from the BPCs to find the true impact position of the particle, hence the true $R$. Alternatively, calorimeter-only information can be used to define the radial distance. One way of doing this is by using the default cluster center to define the radial distance. This is energy weighted by positive energy channels in the cluster in the $x, y$, and $z$ directions. Alternatively, the cluster center can be calculated using the positive energy channels clustered in FCall only. The plots of the average reconstructed energy in the maximum energy cluster as a function of $R$ using these two calorimeter definitions of the cluster center are in Figure 3.52. The shapes of these distributions are not as smooth as when the tracking information is used. The spikes in both figures represent biases towards the channel centers. The 


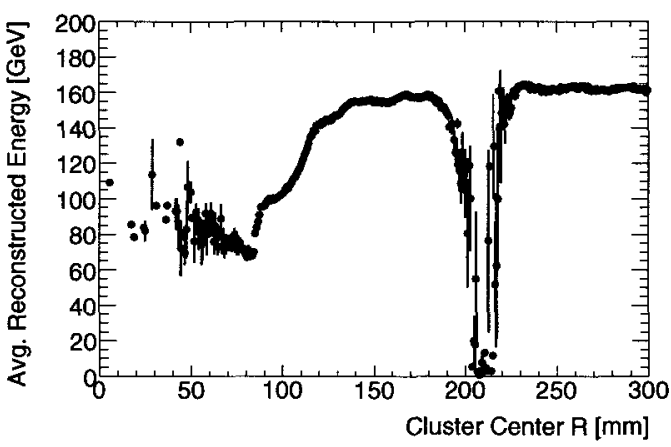

(a)

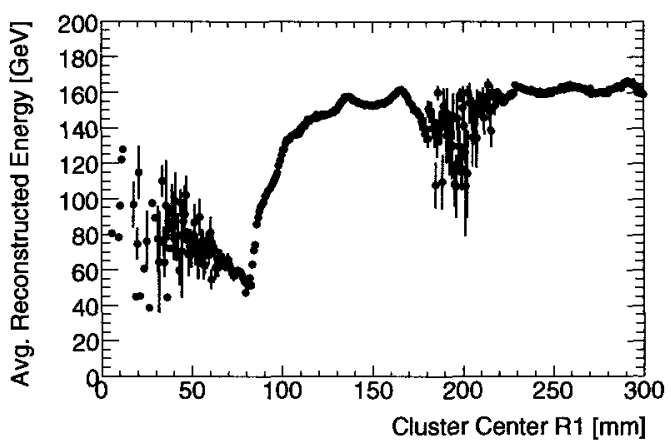

(b)

Figure 3.52: The mean reconstructed energy as a function of radial distance from the center of the beam line for $200 \mathrm{GeV}$ hadrons using the data from impact positions $1,2,3$, and $4 \mathrm{~L}$, using (a) the cluster center as calculated by topological clustering, and (b) only the channels clustered in FCal1.

data in the radial distances from $170 \mathrm{~mm}$ to $220 \mathrm{~mm}$ have large error bars since there are very few cluster centers formed in this region because particles never impact this region of the front face of the calorimeter. However since the cluster center quantity is averaged along all calorimeters in $z$ and the granularity of the channel sampling increases with depth, it is possible that values in this range could be obtained.

\subsubsection{Cluster Correction for Inner Edge Energy Loss}

The distribution presented in Figure 3.51 has a distinct shape and can be used to apply a correction to a cluster once its radial distance from the center of the beam line is known. The radial distance from $50 \mathrm{~mm}$ to $120 \mathrm{~mm}$ has the steepest energy loss with decreasing radius. The energy loss in this region can be fit to the following function:

$$
E(r)=a+\frac{b}{2}\left[1+\operatorname{erf}\left(\frac{r-c}{\sqrt{2} d}\right)\right]
$$

where $r$ is the radial distance, $a$ the offset from zero in energy, $b$ the plateau of energy, $c$ the threshold in $r$ defining the center of the curved region, and $d$ the width of this 
turn on region on either side of the threshold in $r$. The function was fit in the radial range from $55 \mathrm{~mm}$ to $120 \mathrm{~mm}$, to best model the turn-on curve. In the fit $a$ was fixed to zero, to reduce the number of fit parameters and because in theory at $R=0$ the mean reconstructed energy should be near zero. The fit to the data is shown in Figure $3.53(\mathrm{a})$. The fit finds the plateau to be at $b=151.5 \mathrm{GeV}$, which is slightly lower than the peak reconstructed energy due to the upper limit of the fit range. Increasing the fit range beyond $120 \mathrm{~mm}$ results in a poor fit to the data. The fit gives a threshold to be at $c=73.0 \mathrm{~mm}$, close to the inner edge of the FCal at 72.3 $\mathrm{mm}$. The fit for the width was $d=14.5 \mathrm{~mm}$, so over a range of about $30 \mathrm{~mm}$ at the inner-most edge there is a large variation in the possible reconstructed energy of a hadron.

One important aspect to consider for any correction is to determine the energy dependence, if any, of the correction parameters. The function proposed above has an inherent energy dependence, the value of the plateau. However, an alternative function can be used that is normalized by the plateau value, $b$ :

$$
F(r)=\frac{E(r)}{b}=\frac{1}{2}\left[1+\operatorname{erf}\left(\frac{r-c}{\sqrt{2} d}\right)\right] .
$$

This normalized distribution and associated fit are shown in Figure 3.53(b).

Once the fit parameters were obtained the data were corrected by applying the following procedure. For each event the radial distance $R$ to the cluster with the maximum energy was calculated. In the ideal case of the test beam this was done using the projection of the tracks onto the front face of the FCal. This value was substituted into the normalized function defined in Equation $3.35(r=R)$. The function will return a value between 0 and 1 , which represents the fraction of energy with respect to the plateau value. The measured cluster energy $E_{\text {uncorr }}$ was corrected 


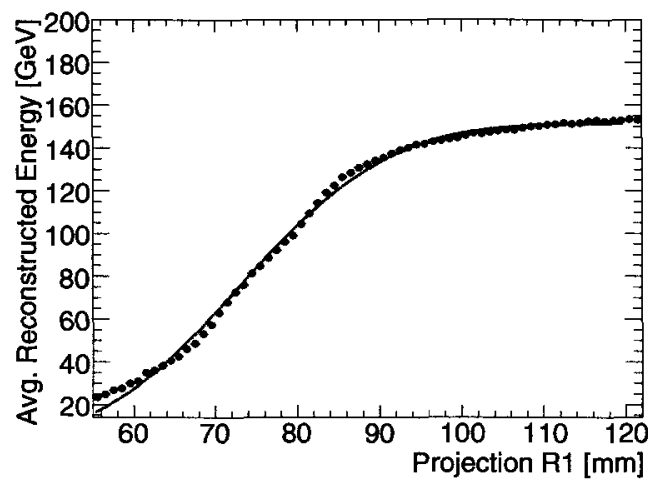

(a)

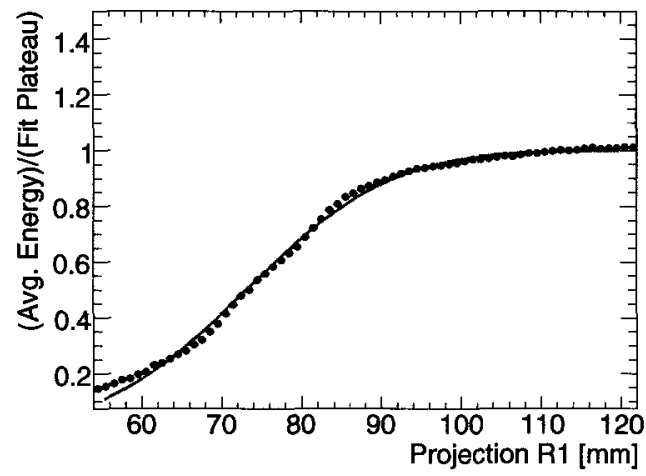

(b)

Figure 3.53: Parameterizing the energy lost at the inner edge of the calorimeter with an error function. Figure (a) shows the fit to the data, where the free parameters are for the plateau, width, and threshold of the curve. Figure (b) is the normalized distribution where the data points have been divided by the fit for the plateau in (a).

according to:

$$
E_{\text {corr }}=\frac{E_{\text {uncorr }}}{F(R)}
$$

The energy distribution for the combined data of impact positions 1, 2, and 3 before and after the correction are shown in Figure 3.54. The mean value of the raw distribution increases closer to the value for the electromagnetic response for $200 \mathrm{GeV}$ hadrons, and there is a decrease in the standard deviation of the distribution.

The uncorrected data cannot be fit to a double Gaussian, because of the low energy tails. But from the raw distributions the mean reconstructed energy and width of the distributions before and after the correction are:

$$
\begin{aligned}
\langle E\rangle_{\text {uncorr }} & =132.6 \mathrm{GeV} \quad \sigma_{\text {uncorr }}=43.3 \mathrm{GeV} \\
\langle E\rangle_{\text {corr }} & =152.8 \mathrm{GeV} \quad \sigma_{\text {corr }}=31.0 \mathrm{GeV}
\end{aligned}
$$

The mean energy is improved by about $13 \%$ and the energy resolution is improved from $32.6 \%$ down to $20.3 \%$. The fraction of events below $50 \mathrm{GeV}$ goes from $7.6 \%$ to $1.2 \%$. The fraction of events above $250 \mathrm{GeV}$ goes from $0.04 \%$ to $2.3 \%$. 

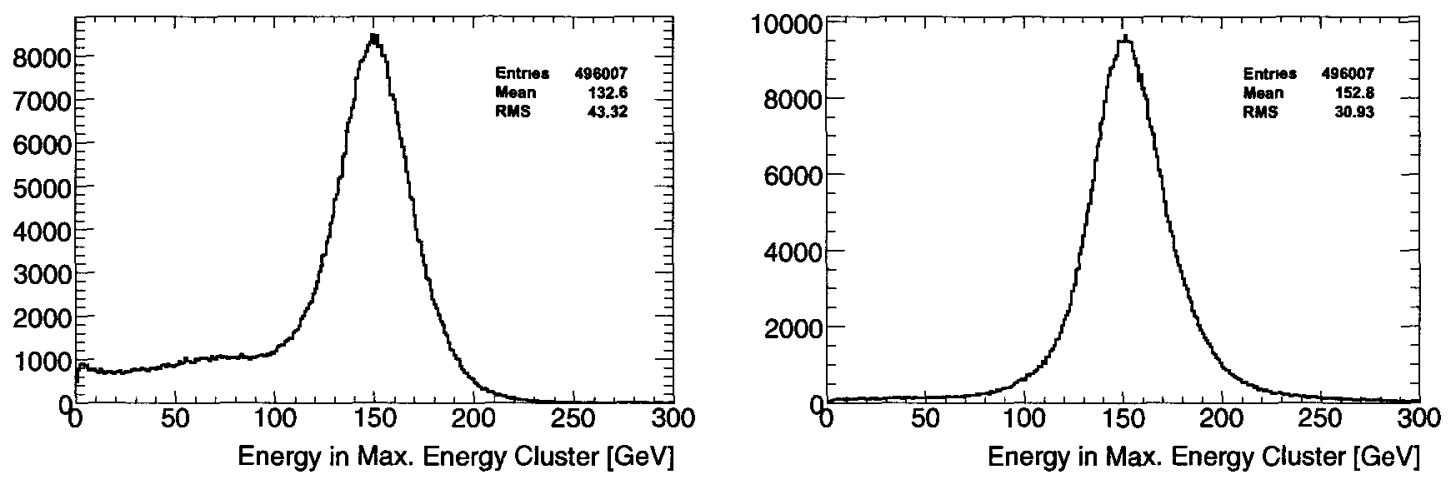

Figure 3.54: Energy distribution for $200 \mathrm{GeV}$ hadrons for position 1, 2, and 3 before (left) and after (right) the inner edge correction, where the radial distance $R$ has been calculated using the BPC projected tracks.

It is necessary to test the energy dependence of the fit parameters $c$ and $d$ in Equation 3.35. MC simulations are required as the inner edge test beam data were only taken for hadrons at an energy of $200 \mathrm{GeV}$. Prior to doing this it is necessary to compare the data and $\mathrm{MC}$ for $200 \mathrm{GeV}$ hadrons at the inner edge.

\subsubsection{Data and Monte Carlo Comparisons of Energy Depo- sition}

MC samples were generated for impact positions 1,2 , and 3 . The average reconstructed energy in the maximum energy cluster as a function of the radial distance from the beam pipe is shown in Figure 3.55 for the data and MC. For the MC the projected radial distance is taken from the true particle generation information. For comparison the data distribution is superimposed as filled circles.

The turn-on curve of the MC is steeper than in the data, reflecting the narrower and denser showers in the MC. The cross-over between the data and $\mathrm{MC}$ is at about $75 \mathrm{~mm}$, which is approximately the inner radius of the FCal.

For particles that strike the front face of FCal1 at $R<75 \mathrm{~mm}$, but interact with 


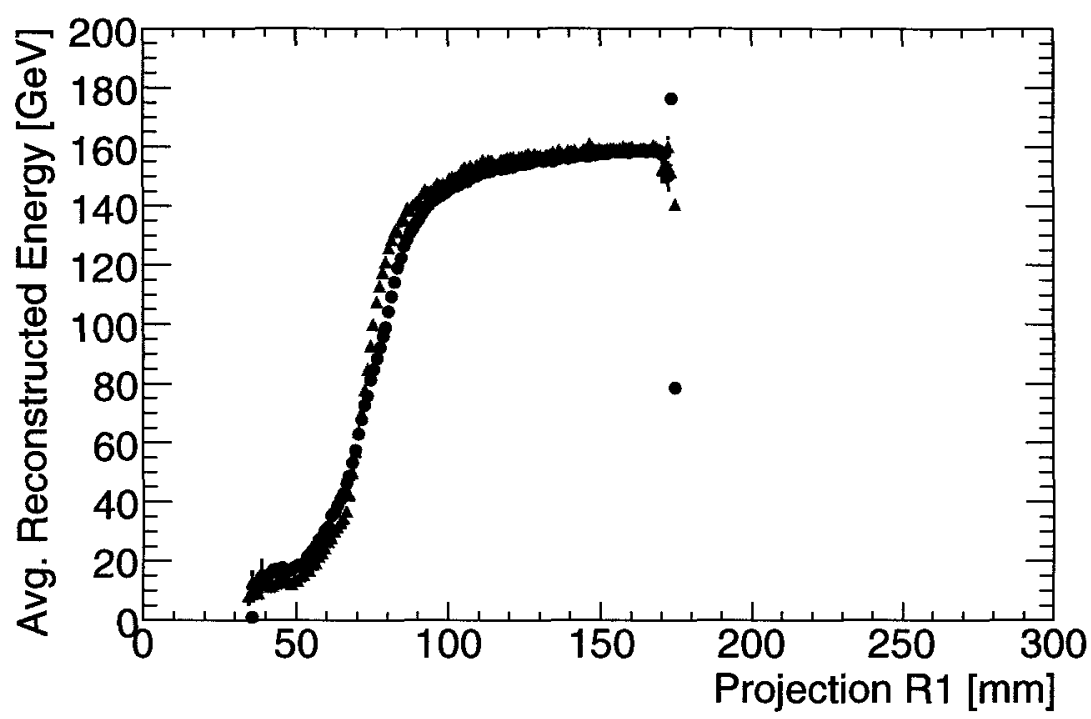

Figure 3.55: The mean reconstructed energy as a function of radial distance from the center of the beam line for $200 \mathrm{GeV}$ hadrons using the data (circles) and MC (lightly shaded triangles) from impact positions 1,2 , and 3.

the material of the beam pipe or liquid argon downstream, the showers that develop in the data will be wider than the MC and will have a higher probability of depositing more energy in the active regions of the calorimeter. The two cases of $R>75 \mathrm{~mm}$ and $R<75 \mathrm{~mm}$ for the $\mathrm{MC}$ and data are demonstrated in Figure 3.56.

The general features of the energy loss as a function of increasing distance to the center of the beam line agree well between data and MC. The goal of the MC is to
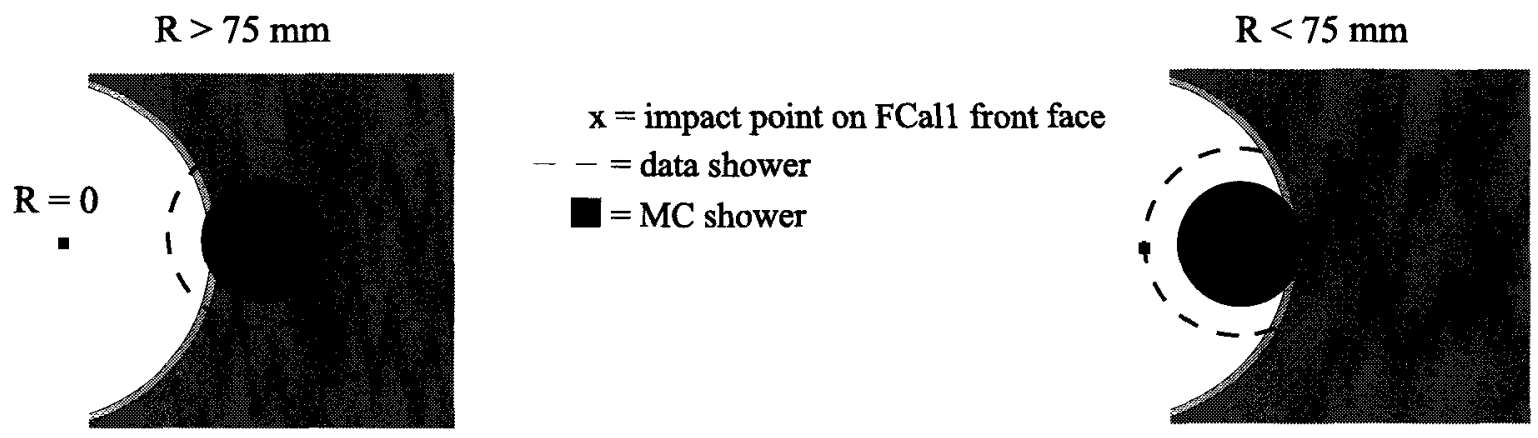

Figure 3.56: Qualitative description of data and MC shower development at the inner edge. 

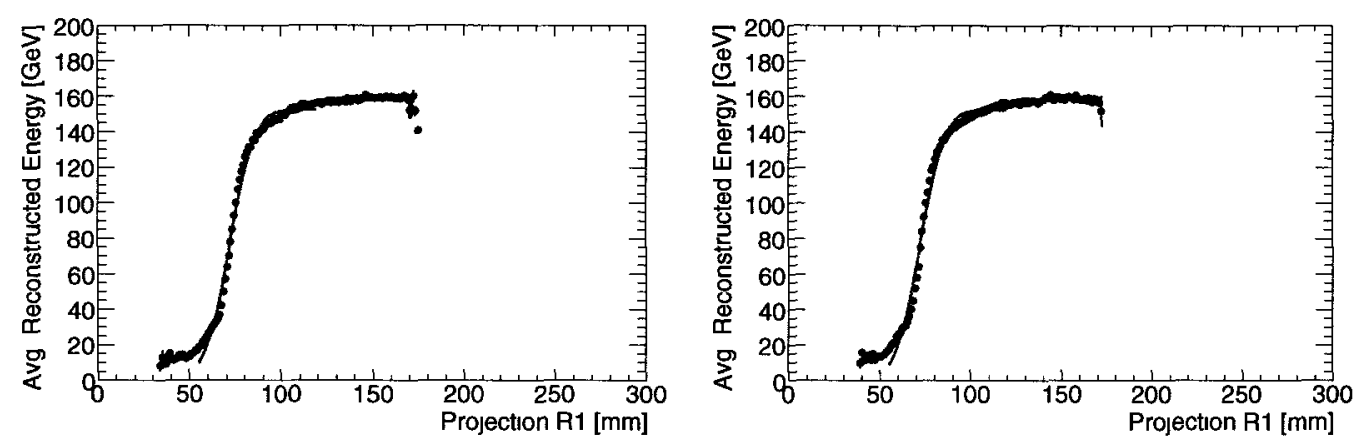

Figure 3.57: The average reconstructed energy versus radial distance from the beam line center for MC with real (left) and square (right) beam profiles.

test if the proposed method of correcting the clusters is energy dependent, and if so, if that dependence can be parametrized.

The default MC simulation in this thesis used the real beam profile. Since there was no data for energies other than $200 \mathrm{GeV}$ for position 1, 2, and 3 a square beam profile was simulated for alternative energies. A comparison is made in Figure 3.57 of MC using the real beam profile versus the square beam profile. The fits to Equation 3.34 for these two samples are virtually identical:

\begin{tabular}{l|c|c} 
& real & square \\
\hline plateau & $152.38 \pm 0.14$ & $152.46 \pm 0.13$ \\
threshold & $71.96 \pm 0.03$ & $72.28 \pm 0.03$ \\
width & $10.85 \pm 0.05$ & $10.85 \pm 0.05$
\end{tabular}

To check if the two parameters in the normalized fit function ( $c$ and $d$ of Equation 3.35) are energy dependent MC data sets were generated using the square beam profile for the inner edge positions 1,2, and 3, for energies (not available with test beam data) of $100 \mathrm{GeV}, 150 \mathrm{GeV}, 250 \mathrm{GeV}, 300 \mathrm{GeV}$, and $400 \mathrm{GeV}$. The energies considered correspond to the energy range of hadrons of interest for ATLAS analyses.

The distributions of the average reconstructed energy as a function of radial dis- 


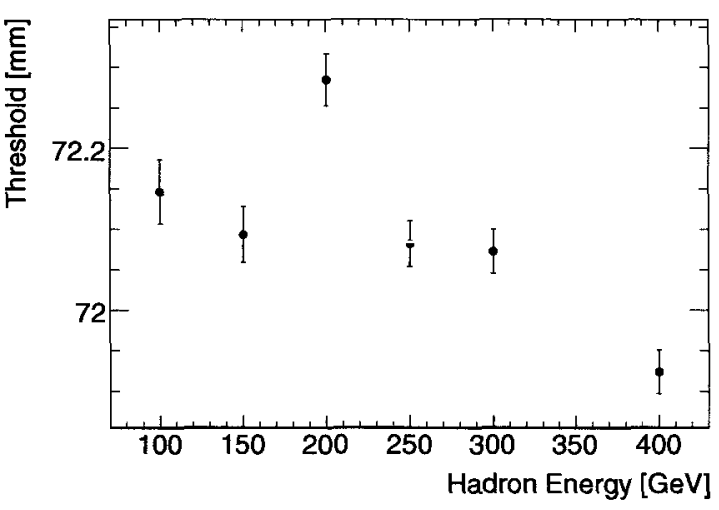

(a)

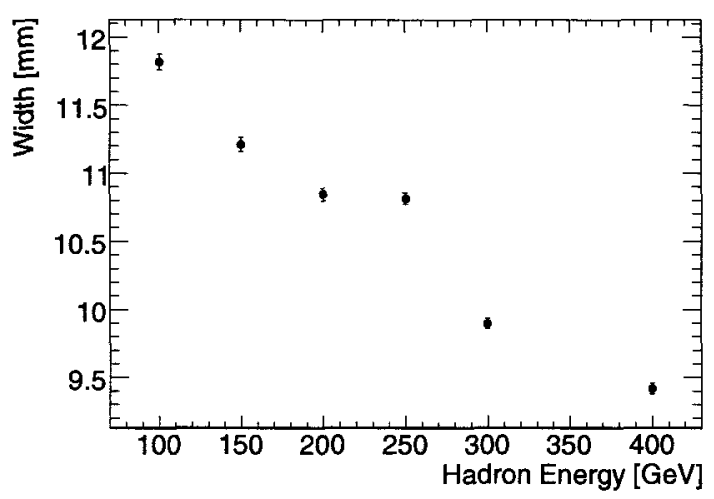

(b)

Figure 3.58: The (a) threshold (parameter c) and (b) width (parameter $d$ ) as a function of hadron beam energy for the inner edge correction function, using $\mathrm{MC}$ simulations of the inner edge positions at a range of energies, where the impact position on the front face of the FCal is calculated using truth information.

tance were fit to the error function, as before. These distributions were then normalized to their plateau values. The fits for the threshold $(c)$ and width $(d)$ as a function of the hadron beam energies are shown in Figure 3.58. In generating these plots the impact point on the front face of FCal1 was calculated using MC truth information.

Figure 3.58(a) shows the fit for the threshold parameter as a function of hadron energy. The distribution is fairly flat, although the $200 \mathrm{GeV}$ and $400 \mathrm{GeV}$ points are slightly away from the average grouping of the other energy points. A linear fit to these points with a slope of zero yields an intercept of $72.1 \mathrm{~mm}$.

The fit for the width parameter as a function of the hadron beam energy is shown in Figure 3.58(b). There is a small energy dependence, with a smaller width for higher energies.

In ATLAS the true impact position of the hadron on the front face of FCall will not be known. One variable that is available is the topological cluster center, calculated for every cluster. If the analysis is redone using the cluster center to calculate the radial distance, the resulting fits for the threshold and width as a function of hadron 


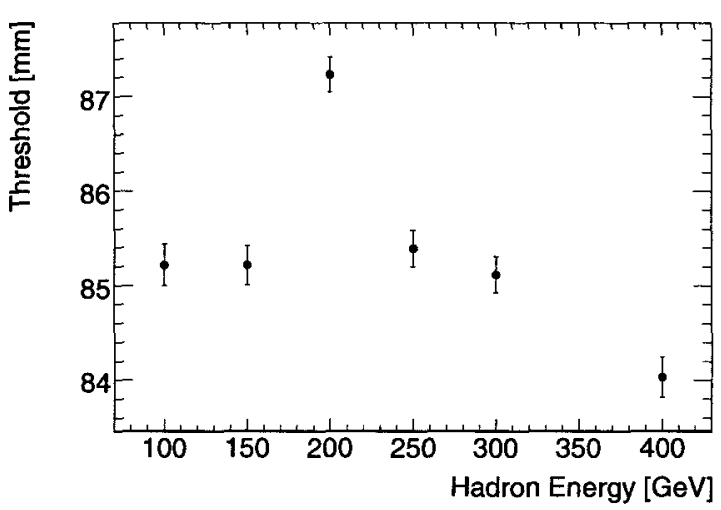

(a)

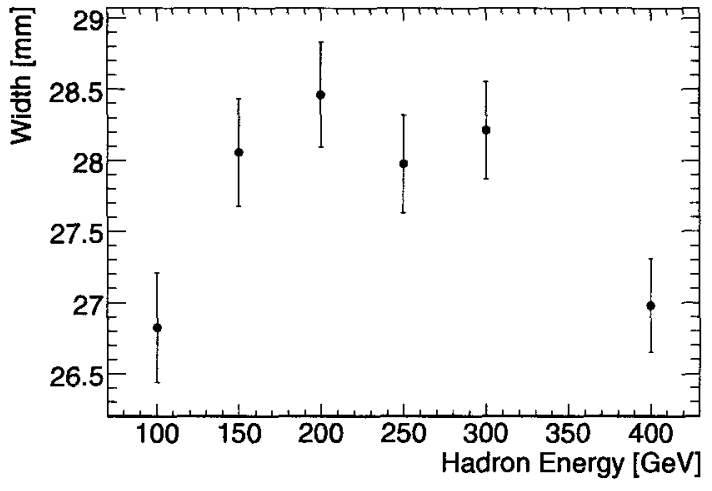

(b)

Figure 3.59: The (a) threshold (parameter $c$ ) and (b) width (parameter $d$ ) as a function of hadron beam energy for the inner edge correction function where the radial distance from the center of the beam line is calculated using the cluster center.

energy are those shown in Figure 3.59.

The uncertainty is larger for these parameters since the distributions of the average reconstructed energy versus radial distance from the center of the beam line are more scattered with biases towards the channel centers (as shown in Figure 3.52(a) for the data).

The threshold (which is the midpoint of the turn-on curve) and width are much larger than when the truth information is used. However, the width is relatively constant across the hadron energies. This flat behavior is likely because it is calculated by averaging over all modules, with a pull towards the peak of the shower.

If we use the average fit results for the threshold and width using the cluster center, and substitute those into the proposed radial correction function, Equation 3.35, this can be used to correct for the energy lost. Using this correction on the $\mathrm{MC}$ for $200 \mathrm{GeV}$ hadrons and calculating $R$ from the cluster center, the overall energy resolution is improved from $36.5 \%$ to $21.3 \%$, and the mean reconstructed energy is improved by $15 \%$.

It is important to note that this method is applicable only under the strict condi- 
tions presented here. It uses the topological cluster with the maximum energy cluster in the event, at the electromagnetic scale. In this analysis the topological cluster splitter has been disabled; the effect of the topological cluster splitter in the context of the inner edge is discussed in the next section. The correction described in this section does not correct for upstream material losses, only losses associated to energy leakage down the beam line and energy outside the maximum energy cluster in the event.

\subsubsection{Data and Monte Carlo Comparisons of Cluster Mul- tiplicities}

In comparing the data and $\mathrm{MC}$ it was shown that the $\mathrm{MC}$ showers are more narrow. This can have a large effect near the inner edge of the FCal, at high $|\eta|$, where showers can deposit energy in the calorimeter all around the beam pipe. The decision to form new clusters from this spray of energy relies on a significant fraction of energy deposition (a channel with energy greater than $4 \sigma$ significance from the noise). Figure 3.60 shows the topological cluster multiplicities in data and $\mathrm{MC}$ for the inner edge hadron data, and position $4 \mathrm{~L}$ for reference. As the particle impact position increases in $|\eta|$ the cluster multiplicity increases. This is due to new clusters being formed around the beam pipe isolated from other clustered channels.

In Section 3.9.3 it was shown that if the default topological cluster splitter was enabled the event multiplicities significantly increased for hadron events. Figure 3.61 shows this same trend for position $1,2,3$, and $4 \mathrm{~L}$ for $200 \mathrm{GeV}$ hadron data and MC using the topological cluster splitter. There is a larger discrepancy between data and MC with increasing $|\eta|$. This is likely due to the narrow shower shapes in the MC that do not deposit enough significant energy around the beam line to seed new 


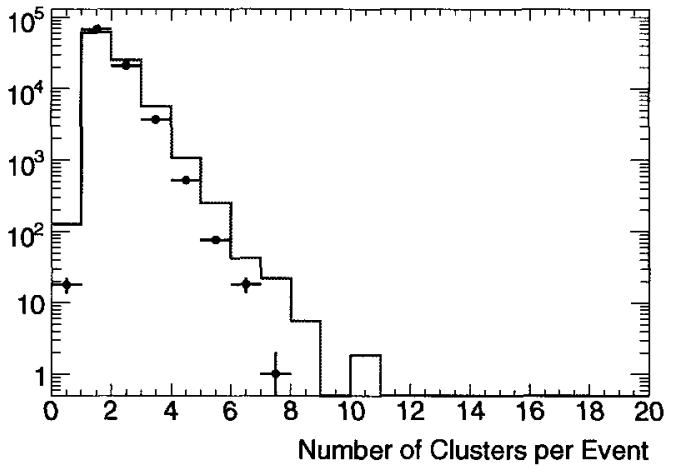

(a) Position 4L $(|\eta| \sim 3.7)$

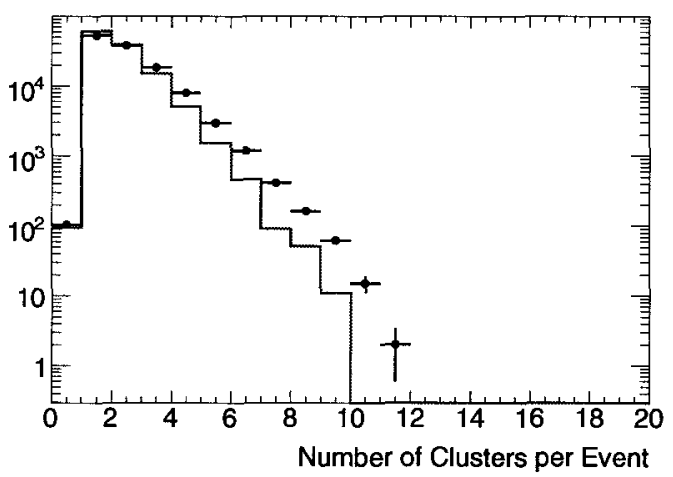

(c) Position $2(|\eta| \sim 4.5)$

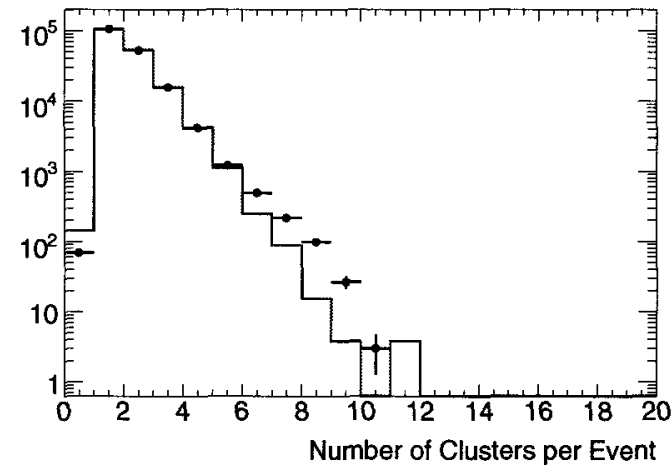

(b) Position $3(|\eta| \sim 4.3)$

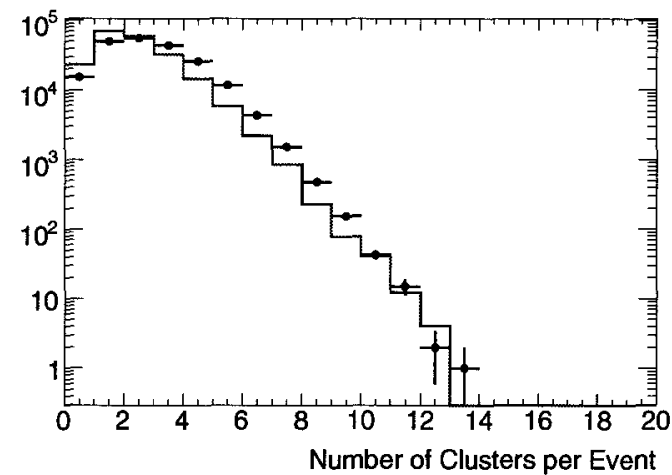

(d) Position $1(|\eta| \sim 4.9)$

Figure 3.60: Cluster multiplicity for the data (points) and MC (line) at increasing values of $\eta$. 


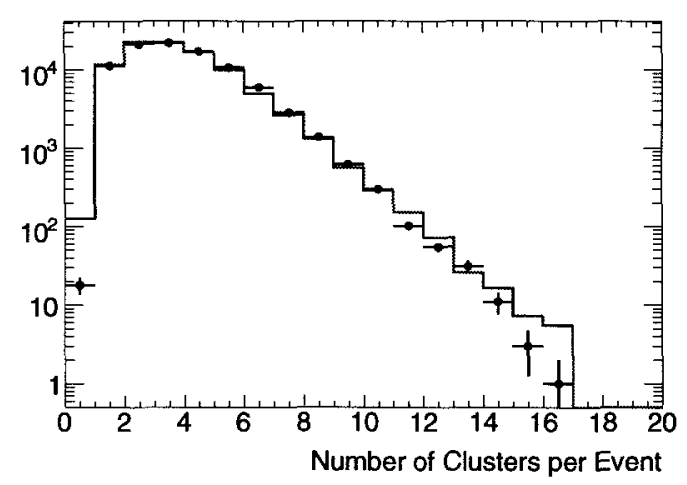

(a) Position 4L $(|\eta| \sim 3.7)$

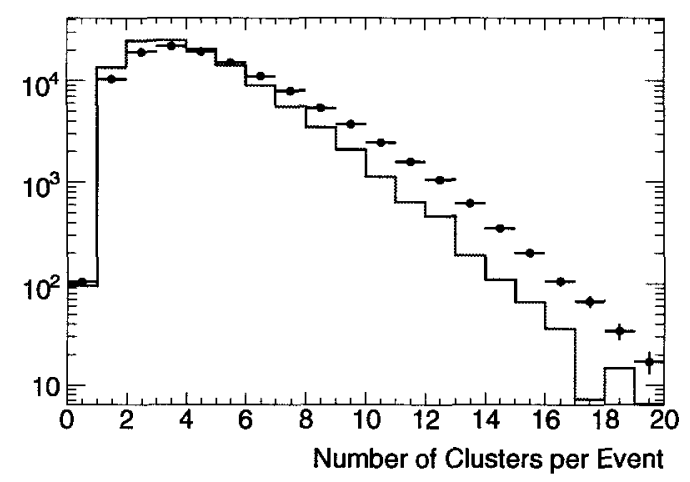

(c) Position $2(|\eta| \sim 4.5)$

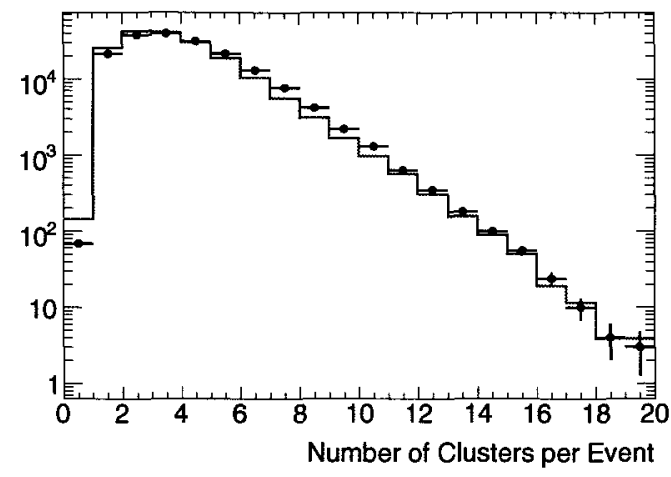

(b) Position $3(|\eta| \sim 4.3)$

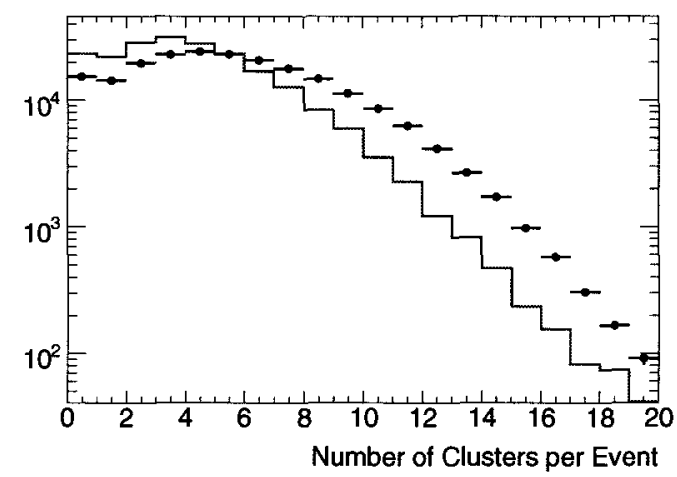

(d) Position $1(|\eta| \sim 4.9)$

Figure 3.61: Cluster multiplicity for the data (points) and MC (line) at increasing values of $\eta$ by applying the topological cluster splitter.

clusters. The proposed correction to energy cut from $500 \mathrm{MeV}$ to $1 \mathrm{GeV}$ to improve the performance of the topological splitter algorithm (Section 3.9.3) was motivated by the average electronic noise in the channels in the FCal. However, this suggestion of modifying the energy cut for the FCal would not correct the discrepancy observed between the data and $\mathrm{MC}$ for the inner edge.

\subsubsection{Summary}

The inner edge of the FCal is a complicated region of the ATLAS calorimeter system, as it is at the highest $|\eta|$ range and suffers from shower leakage. The interaction of particles with the various components, both inactive and active, is difficult to model. 
It has been observed with the test beam data that showers that develop near the inner edge tend to extend around the beam pipe, not just the side of initial particle impact.

\subsection{Summary of the Forward Calorimeter Test Beam Analysis}

A beam test was performed on the FCal using electrons and hadrons with energies in the range of approximately $10 \mathrm{GeV}$ to $200 \mathrm{GeV}$. Using this test beam data the predicted conversion between the detector response in ADC counts and energy for FCall was verified. The intrinsic energy resolution of the calorimeter to electrons and hadrons was found to be within the ATLAS requirements for the ideal location in the FCal with minimal upstream material and optimized test beam clustering.

Using the topological clustering algorithm, which is default in ATLAS, the configuration for electrons and hadrons that optimizes the energy resolution and linearity was found to be $S / N / P=4 / 2 / 0$. Topological cluster moments were examined and give confidence that the likelihood of distinguishing electrons and hadrons will be possible using shower shape variables alone. These shower shapes follow the predicted properties of hadronic and electromagnetic particle interactions with matter. An investigation of the topological cluster splitting routine revealed the need to increase the default energy cut parameter from the current $500 \mathrm{MeV}$ to at least $1 \mathrm{GeV}$ in the FCal.

$\mathrm{MC}$ simulations of the test beam were performed and results indicate the MC and data response at the electromagnetic scale agree within $2 \%$, which is within the systematic errors studied for the data. Despite this good agreement, the MC showers 
tend to be too dense (by about 14\%), too narrow (by about 10\%), and too short (by about $5 \%)$ as compared to the data for high energy hadrons $(\geq 60 \mathrm{GeV})$.

The characteristics of energy loss at high $|\eta|$ near the beam pipe is modelled well in the MC, but as the MC showers are too dense and narrow this causes small differences in the energy loss versus radial distance from the beam line center. The $\mathrm{MC}$ also produces fewer clusters per event than seen in the data at large values of $|\eta|$. A correction to account for energy losses as a function of radial distance was suggested, and improves the energy resolution by about $13 \%$ and reconstructed energy by about $12 \%$ for particles which strike FCal1 at radial distances less than $120 \mathrm{~mm}$ from the center of the beam pipe.

This test beam analysis could be improved by putting more of the features from the data into the $\mathrm{MC}$, such as using the run-by-run and channel-by-channel electronic noise, the real particle tracks, and/or the real beam content including contamination. It would be interesting to calibrate the downstream tail catcher to measure the leakage out of the back or at the inner edge of the FCal. One could apply the shower shape variables as a discriminator for electrons and hadrons to further remove contamination. The analysis could also be repeated for different GEANT4 hadronic physics lists to find the combination of models that best reproduces the data.

Comparison of the energy resolution obtained with data and MC, and the effect of upstream material (position $4 \mathrm{~L}$ versus position $4 \mathrm{H}$ ) is the subject of another ongoing analysis by P. Thompson (University of Toronto). 


\section{Chapter 4}

\section{Determination of the Top Quark Pair Cross-section in ATLAS}

The top quark was first discovered in 1995 at the Tevatron by the CDF and D0 collaborations $[43,44]$. It is the most massive quark, with a mass 35 times that of the bottom quark, the next most massive quark, and plays a particularly important role in the SM, Higgs discovery, and potentially in physics beyond the SM. This chapter opens by reviewing the theoretical importance of the top quark and the current experimental constraints on its properties. It then describes the physics potential of top quark in the ATLAS experiment.

This analysis uses Monte Carlo (MC) samples to study the cross-section of top and antitop pairs produced at the LHC, by studying the decay into final states containing two leptons, two neutrinos, and two $b$ quarks. The primary goal of this work is to study the possible triggers that can be used for the analysis, and propose a method by which variations in the trigger efficiency can be introduced into the cross-section calculation. 


\subsection{The Role of the Top Quark in the Standard Model}

The SM has several parameters that cannot be predicted theoretically and must be measured through experiment. Some of these parameters are ${ }^{1}$ :

- 12 Yukawa couplings,

- the gauge couplings $g_{s}\left(S U(3)_{C}\right), g\left(S U(2)_{L}\right)$, and $g^{\prime}\left(U(1)_{Y}\right)$,

- two parameters of electroweak symmetry breaking: $v, m_{H}$.

Precise measurements of these parameters are used to test the SM and constrain theories that reach beyond it. Values for $v$ can be extracted from studying electromagnetic coupling; it is found to be $v=246 \mathrm{GeV}$ [1]. Recall that the minimum value of the Higgs potential was $v / \sqrt{2}$ which equals approximately $173.9 \mathrm{GeV}$, and is the scale where electroweak symmetry breaks. Note this is roughly the mass of the top quark.

The couplings $g$ and $g^{\prime}$ are related to the electromagnetic coupling constant, and can be calculated using low energy scattering experiments. Recall that the mass of the $Z$ and $W$ bosons were generated in electroweak symmetry breaking and are related to the coupling constants (Equation 1.6). The mass of the $Z$ boson was precisely measured at the LEP experiment at CERN by colliding $e^{+} e^{-}$pairs with center of mass energies around the $Z$ resonance.

The SM $W$ and $Z$ boson masses are related by $M_{W}=M_{Z} \cos \theta_{w}$, where $\theta_{w}$ is called the weak mixing angle. This angle can be extracted from electroweak scattering experiments. At LO the $W$ boson mass can be written in terms of the measurable

\footnotetext{
${ }^{1}$ Not included in this list or discussed in this thesis are the eight parameters of the weak charged current flavour mixing for the quark (Cabibbo-Kobayashi-Maskawa) matrix $[45,46]$ and the beyond SM lepton (Pontecorvo-Maki-Nakagawa-Sakata) matrix $[47,48]$.
} 
quantities:

$$
M_{W}^{2}=\frac{\pi \alpha}{\sqrt{2} G_{F}} \frac{1}{\sin ^{2} \theta_{w}}
$$

where $G_{F}$ is the Fermi constant and is related to the vacuum expectation value by $G_{F}^{-1}=\sqrt{2} v$. The variable $\alpha$ is the electromagnetic coupling constant. At one-loop there is a correction to the $W$ mass that comes from self-energy corrections from virtual fermions produced in the vacuum, shown in Figure 4.1. The correction to the $W$ mass enters as a parameter $\Delta r$ :

$$
M_{W}^{2}=\frac{\pi \alpha}{\sqrt{2} G_{F}} \frac{1}{\sin ^{2} \theta_{w}(1-\Delta r)} .
$$

For the top quark, the contribution to the correction is:

$$
\Delta r^{\mathrm{top}}=\frac{-3 G_{F} m_{t}^{2}}{8 \sqrt{2} \pi^{2}} \frac{1}{\tan ^{2} \theta_{w}}
$$

where $m_{t}$ is the mass of the top quark (similar expressions exist for the other fermions). As the top quark mass is significantly larger than the fermions, it has the largest contribution to the radiative $W$ mass correction.

The Higgs boson also contributes to the $W$ self-energy as shown in Figure 4.2 and adds an additional correction to the mass of the $W$ by:

$$
\Delta r^{\mathrm{Higgs}}=\frac{11 G_{F} M_{Z}^{2} \cos ^{2} \theta_{w}}{24 \sqrt{2} \pi^{2}} \ln \left(\frac{M_{H}^{2}}{M_{Z}^{2}}\right) .
$$

The correction to the $W$ mass is only logarithmic with the Higgs boson mass, but is proportional to the mass of the top quark squared. Turning the equations around slightly, precise measurements of the top quark and $W$ boson masses make it is possible to put constraints on the mass of the Higgs boson. 


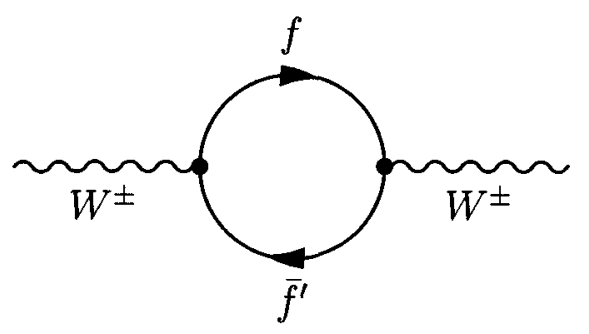

Figure 4.1: Feynman diagrams showing the one-loop correction to the $W$ boson mass arising from fermions in the SM. For the $W$ boson the loop $f$ is a fermion and $\bar{f}^{\prime}$ can be any of the charged weak fermion partners to $f$.
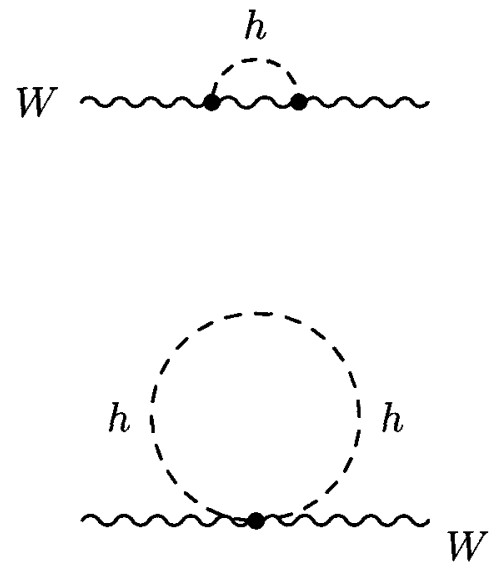

Figure 4.2: Feynman diagrams showing the one-loop correction to the $W$ boson mass arising from the Higgs boson.

The top quark plays an important role in the indirect evidence for the Higgs boson by its contributions to the $W$ mass. The large mass of the top also implies that it has the strongest fermion coupling to the Higgs boson, so evidence of the Higgs boson could come by studying production rates and decay chains of the top quark.

\subsubsection{Top Quark Production}

In a hadron collider, top quark pair $(t \bar{t})$ production is dominated by $q \bar{q}$ annihilation, gluon-gluon fusion, or by quark-gluon interaction. In all cases of $t \bar{t}$ production the initial state particles, called partons, must have enough energy to produce two massive 

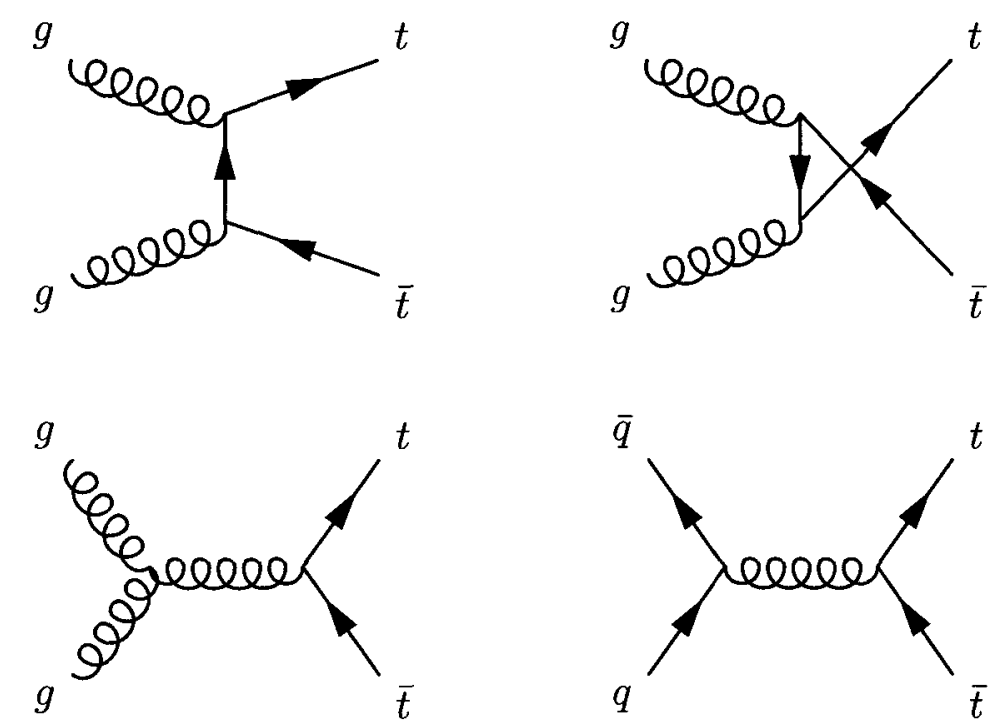

Figure 4.3: Leading order Feynman diagrams for the production of top quark pairs at the LHC.

top quarks. The Feynman diagrams for the production of $t \bar{t}$ at the LHC for leading order (LO) and some of the possible next-to-leading order (NLO) processes are shown in Figures 4.3 and 4.4. For higher order (> LO) processes, top pairs are produced in association with at least one other particle, typically a gluon or quark.

Calculating the cross-section for hadron interaction is complicated and is based on the factorization of QCD into two elements. The first step involves understanding the number and types of partons composing the initial hadron, and the fraction of the total momentum carried by each. The probability for a parton $a$ coming from a hadron $A$ to have a fraction of the total momentum $x_{A}$ is defined as $f\left(x_{A}\right)$. This is called the parton distribution function (PDF). PDFs are models that are tuned from experiment, by making global fits to parameters of cross-section measurements involving hadrons. The second step involves calculation of high energy collisions, such as those depicted in Figures 4.3 and 4.4, where the colliding partons which carry some fraction of the initial hadron energy, are considered free. Perturbative QCD can be used because the strong coupling constant is small at short distances. 

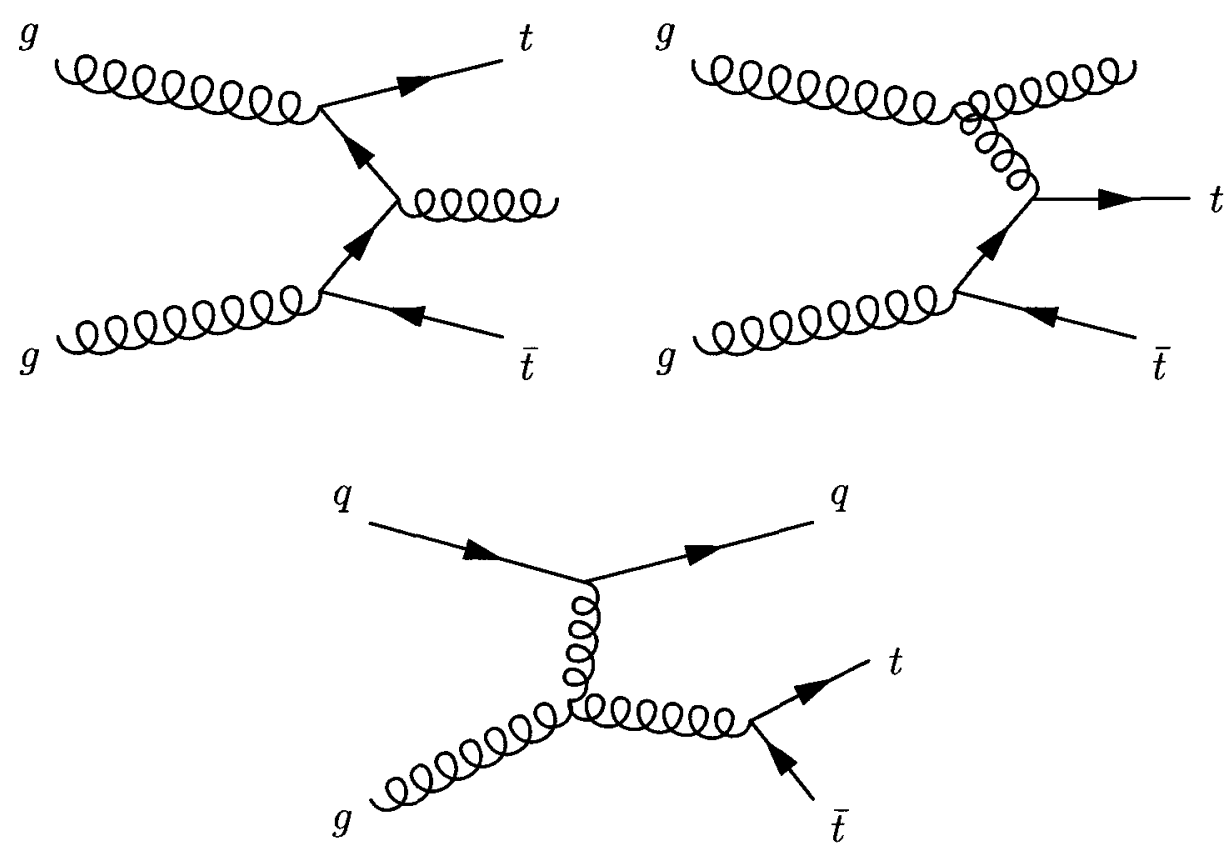

Figure 4.4: Next to leading order Feynman diagrams for the production of top quark pairs at the LHC.

There are currently two high energy colliders that have sufficient center of mass energies to produce top quarks: the Tevatron at Fermilab and the LHC at CERN. The production cross-section for top quark pairs at each of these facilities is shown in Table 4.1 for the top quark with mass of $172.5 \mathrm{GeV}$. For the LHC there are three center of mass energies listed, reflecting the foreseen energy steps of the collider. The LHC is currently colliding protons at a center of mass energy of $7 \mathrm{TeV}$. The crosssections at the LHC are significantly greater than at the Tevatron, allowing for more detailed studies of the top quarks.

\subsubsection{Top Quark Decays}

Once the top quark has been produced, in pairs or singly, it can decay via a variety of channels, as represented by the Feynman diagram in Figure 4.5. Approximately $100 \%$ of the time a top quark will decay into a $W$ boson and $b$ quark. The $b$ quark 


\begin{tabular}{|c|c||c|c|c|}
\hline \hline & $\begin{array}{c}\text { Tevatron }(p \bar{p}) \\
\sqrt{s}=1.96 \mathrm{TeV}\end{array}$ & $\begin{array}{c}\text { LHC }(p p) \\
\sqrt{s}=14 \mathrm{TeV}\end{array}$ & $\begin{array}{c}\text { LHC }(p p) \\
\sqrt{s}=10 \mathrm{TeV}\end{array}$ & $\begin{array}{c}\text { LHC }(p p) \\
\sqrt{s}=7 \mathrm{TeV}\end{array}$ \\
\hline$\sigma_{t \bar{t}}$ & $7.3 \mathrm{pb}$ & $886.3 \mathrm{pb}$ & $402.5 \mathrm{pb}$ & $160.8 \mathrm{pb}$ \\
\hline \hline
\end{tabular}

Table 4.1: The theoretical cross-sections for $t \bar{t}$ production at the Tevatron $(p \bar{p}$ at $\sqrt{s}=1.96$ $\mathrm{TeV}$ ) and the LHC ( $p p$ at $\sqrt{s}=7 \mathrm{TeV}, 10 \mathrm{TeV}, 14 \mathrm{TeV}$ ), based on the next-to-next-toleading order (NNLO) by [49].

will form hadronize resulting in colour connected hadrons, forming what is called a jet. The $W$ boson can decay into two quarks $q \bar{q}^{\prime}$ resulting in more particle jets, or lepton-neutrino pairs. In $t \bar{t}$ events there will be two $W$ bosons produced, if both decay to quark-antiquark pairs there will be four quark jets in addition to the two $b$-jets, this decay channel is called the fully hadronic channel. If one $W$ boson decays to quark pairs and the other to leptons then the event is a semi-leptonic decay. If both $W$ bosons decay to leptons the event is denoted by the dilepton channel. The branching ratios for each of these three $t \bar{t}$ decay channels are shown in Table 4.2.

The dilepton channel in which the charged leptons in the final state are either electron or muon type, is the signature of interest for this thesis. In the ATLAS detector the dilepton channel can be identified by the presence of two (oppositely) charged leptons $(e e, \mu \mu, e \mu)$, at least two jets and missing energy from the two neutrinos escaping without interaction. For the remainder of this thesis the term dilepton channel refers to this signal. Although selection criteria will be applied to identify these events, $W$ decays into a $\tau$ lepton which itself decays into leptons will also be considered, although the event selection cuts will not be tuned explicitly to search for this decay. 


\begin{tabular}{|l|c|r|}
\hline$t \bar{t} \rightarrow(W b)(W \bar{b}) \rightarrow$ & Branching Ratio & Naming Convention \\
\hline \hline$(q \bar{q} b)(q \bar{q} \bar{b})$ & $45.7 \%$ & fully hadronic \\
$(q \bar{q} b)(l \bar{\nu} \bar{b}),(\nu \bar{l} b)(q \bar{q} \bar{b})$ & $43.8 \%$ & semi-leptonic \\
$(l \bar{\nu} b)(\nu \bar{l} \bar{b})$ & $10.5 \%$ & dileptonic \\
\hline \hline
\end{tabular}

Table 4.2: Branching ratios for the different $t \bar{t}$ decay modes, assuming $W \rightarrow l \nu=10.8 \%$ for each $l[1]$.

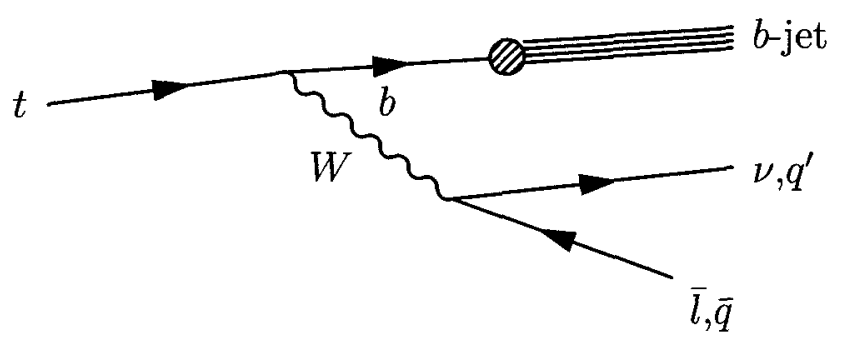

Figure 4.5: Feynman diagram for the decay chain of the top quark. Here $q^{\prime}=c, u$ with corresponding partners $\bar{q}=\bar{s}, \bar{d}$, and $\bar{l}=\bar{e}, \bar{\mu}, \bar{\tau}$ with weak partners $\nu=\nu_{e}, \nu_{\mu}, \nu_{\tau}$. For $\bar{t}$ decays the particles and antiparticles are reversed.

\subsubsection{Current Status of Experimental Results}

The top quark has been directly observed at the Tevatron and very recently at the LHC, so all measurements of top properties have come from the results of these colliders. By combining the results from the two experiments at the Tevatron the top mass is found to be $m_{t}=173.1 \pm 1.3 \mathrm{GeV}[50]$. With this latest result, combined with other SM electroweak precision measurements from SLD, LEP, and the Tevatron, the SM Higgs boson is expected to have a low mass of $115_{-40}^{+58} \mathrm{GeV}$ [51], which is well within the range observable at the LHC.

The top pair cross-section has been measured by both Tevatron experiments, for the different physics channels listed in Table 4.2. Combining the results from the three different decay modes (dileptonic, semileptonic, fully hadronoic) the CDF 
collaboration finds a top pair cross-section of $\sigma_{t \bar{t}}\left(m_{t}=172.5 \mathrm{GeV}\right)=7.50 \pm 0.48 \mathrm{pb}$, consistent with the SM theoretical predictions (Table 4.1) [52]. The D0 experiment finds similar results, consistent with SM expectations.

\subsection{Monte Carlo Data Sets}

MC simulations are used to study the triggering and event reconstruction in ATLAS, and to calculate the expected event yields for $t \bar{t}$ signals and backgrounds. This study is for events at the LHC with $\sqrt{s}=7 \mathrm{TeV}$.

\subsubsection{Generators}

The generation of MC datasets is a complex process, with several steps that involve complicated calculations and modelling. The following describes the general steps for generating MC samples:

1. Matrix Element: Calculate the matrix element for each process to some order in QCD. There are many software packages available to do this. This step calculates the physics signal of interest, in this case $t \bar{t}$ production from the partons of a $p p$ collision (using the PDFs).

2. Parton Shower: The partons from the hard interaction are coloured and radiate gluons as they separate. This sea of colour is described by a parton showering program, motivated by QCD theory. It does not depend on the physics process that generated the initial quarks.

3. Hadronization: The constituents of the parton showers above hadronize to form 
colour-less hadrons, and create particles that are ultimately detected in the detector. The hadronization process is described by models.

4. Underlying Event: The MC must also handle the underlying event, which is the remainder of the non-hard scattered partons of the original interacting protons. The protons collide as colourless objects, then the hard-scattered partons remove colour, and so the remanents of the proton (what is left of it - not exactly a proton anymore by definition) are coloured and the interaction between these coloured states needs to be described. The underlying event is modelled, and is the least understood in the MC generation process. As the interactions of the underlying partons are likely dependent on the energy of the partons, it requires good understanding of the partonic content (described by the PDFs). In ATLAS the underlying event is typically modelled by the JIMMY program.

5. Detector Simulation: The resulting hadrons travel through the detecting medium; these interactions are simulated using GEANT4. This package uses a combination of calculable cross-sections of particles with matter and models based on experimental data.

6. Reconstruction: The reconstruction algorithms are applied to the MC. They are the same software packages used to reconstruct real ATLAS data, clustering regions of potentially interesting physics based on the detector response and applying criteria to define different particle types.

As a final step, some MC generators also simulate the interactions between the other particles in the beam crossing, yielding pileup. The amount of pileup depends 
on the instantaneous luminosity of the colliding beams, which is discussed more in Section 4.7.3. The study in this dissertation is done assuming low luminosity (the early stages of LHC operation), where pileup effects are considered negligible.

Calculating the matrix element in the first step is done to a certain order, most generators consider only the LO, sometimes with additional activity from radiating gluons or quarks. However at the center of mass energies at the LHC there is a lot of energy available to produce top quarks at higher order, therefore it is important to include the full NLO terms for analyses involving the top quark. The ratio of the LO to NLO cross-section for $t \bar{t}$ is approximately 0.67 for the LHC $(\sqrt{s}=14 \mathrm{TeV})$ [49]. Two generators that are used in ATLAS to calculate the matrix elements at NLO for $t \bar{t}$ production are MC@NLO [53] and POWHEG [54]. For some processes where the ratio of the cross-section at some order to a higher order is small, a constant factor called the $k$-factor is applied to the expected cross-section of the MC samples. This ensures the final number of MC predicted events resembles reality (which is to infinite order). The shapes of the kinematical distributions are approximately the same at higher orders, so it is acceptable to multiply by a constant factor.

The MC generator MC@NLO computes the matrix elements for the LO and NLO processes, and feeds the output into the parton showering algorithm. A process at LO that emits radiation at the parton shower level may reproduce a process that is already accounted for at the NLO calculation. To avoid duplicate events the MC@NLO generator assigns some events a weight of -1 , all other events have a weight of +1 . This weight must be taken into account when computing the observed number of events and when examining observable quantities (and must be propagated to the errors appropriately). The generator must communicate the weighting factor to the parton shower, and to date for this reason MC@NLO can only be interfaced to HERWIG [55].

POWHEG employs an alternative technique to avoid double counting between 
NLO events that are identical to events produced by LO plus radiation. When it generates an event at NLO it defines a threshold cutoff equal to the momentum of the hardest emission. In the parton shower any emissions that have energy greater than this threshold are vetoed. All events with the POWHEG generator have a weight of +1 and it can therefore be interfaced with any parton shower following the standard Les Houches format [56]. In ATLAS two POWHEG samples are available with parton showering from PYTHIA and HERWIG.

\subsubsection{Signal Samples}

The MC samples used in this thesis have been centrally produced by the ATLAS collaboration. ${ }^{2}$ GEANT4 v4.9.2 with the QGSP_BERT physics list (described in more detail in Section 3.10) was used for simulation of particle interactions with the detector. The trigger hypotheses were available for each event with the trigger configuration established for early ATLAS data.

In the ATLAS top working group the default matrix element generator for $t \bar{t}$ cross-section measurements is MC@NLO with parton showering from HERWIG and JIMMY for the underlying event. The signal samples for the dilepton $t \bar{t}$ analysis are listed in Table 4.3. The $k$-factors shown are the ratio of the matrix element generator values (calculated at NLO for MC@NLO and POWHEG, and LO for AcerMC) to those calculated at NNLO, based on the calculations by [49] for $7 \mathrm{TeV} p p$ collisions (Table 4.1). The branching ratio for the decay of $W \rightarrow l \nu$ in MC@NLO is 0.111 and for the POWHEG generator is 0.108 , thus the numbers for the cross-sections and $k$-factors are slightly different.

Along with the slightly different cross-section the different MC signal samples use

\footnotetext{
${ }^{2}$ The MC samples have been simulated using Athena 15.6.x and digitized/reconstructed using Athena 15.6.9.8, and are the so-called ' $\mathrm{mc08} 7 \mathrm{TeV}$ ' samples.
} 


\begin{tabular}{|c|c|c|}
\hline \hline Matrix Element Generator + Parton Shower & $\sigma[\mathrm{pb}]$ & $k$-factor \\
\hline MC@NLOv3.1 + HERWIG & $\mathbf{8 0 . 2 0 1}$ & $\mathbf{1 . 0 9}$ \\
\hline POWHEG + HERWIG v6.510 & 79.118 & 1.10 \\
POWHEG + PYTHIA v6.417 & 79.118 & 1.10 \\
AcerMC v3.5 + HERWIG/JIMMY & 57.98 & 1.50 \\
\hline
\end{tabular}

Table 4.3: The top MC signal samples used in the analysis. The default sample is the first one listed in bold font, the other samples (below the line) are the signal samples used in the evaluation of systematics. These are generated with alternative generators and/or parton showering algorithms. The cross-sections and $k$-factors are based on NNLO calculations by [49]. All of the samples only include $t \bar{t}$ decays to the semi-leptonic and dileptonic final states at the appropriate branching ratios (the fully hadronic channel is not included in these samples). In all cases a top mass of $172.5 \mathrm{GeV}$ was assumed. The cross-section $\sigma$ is the effective cross-section of the sample after the generator filter efficiency has been multiplied by the generator cross-section (for example, in these samples the generator is filtered to produce only events from all but the fully hadronic channel).

different parton shower models, which can yield slightly different kinematical distributions. Figures 4.6 to 4.9 show some generator-level distributions of selected kinematical quantities for the MC@NLO, POWHEG+HERWIG, POWHEG+PYTHIA, and AcerMC top signal samples in the dilepton channel (generated with $m_{t}=172.5$ $\mathrm{GeV}$ ). These plots demonstrate the subtle differences between the MC samples that will later be used to asses systematics in the cross-section measurement. In each set of figures the larger plot at the top shows the normalized distributions, and the small figure below shows the ratio of the three alternative MC samples with respect to the default MC@NLO sample. In these plots, and the following discussion, references are made to momentum and energy in the transverse plane $p_{\mathrm{T}}, E_{\mathrm{T}}$. The transverse plane is along the $x$ and $y$ axis (the beam lies along the $z$ axis). The transverse momentum 
and energy are defined as: ${ }^{3}$

$$
\begin{aligned}
& E_{\mathrm{T}}=E \sin \theta \\
& p_{\mathrm{T}}=|\vec{p}| \sin \theta=\sqrt{p_{x}^{2}+p_{y}^{2}} .
\end{aligned}
$$

As a reminder in the following all quantities are shown at truth level.

The true $p_{\mathrm{T}}$ and $\eta$ distribution of the top quarks are shown in Figure 4.6(a) and (b), respectively. There are two top quarks per event; for each top quark the variables shown reflect the final state top quark after any and all radiation has taken place and before its decay into a $W$ boson and $b$ quark. The NLO samples agree well with each other. The AcerMC sample, the only LO matrix element generator has, on average, top quarks with higher $p_{\mathrm{T}}$ than the NLO generators. At NLO the initial energy of the parton is shared between not only the top quark pairs, but in some cases also an additional radiated gluon or quark. The POWHEG+HERWIG sample tends to have a larger fraction of low $p_{T}$ tops than the MC@NLO sample. The $\eta$ distribution of the top quarks shows a larger discrepancy between the LO and NLO generator, with the LO process having more top quarks in the central region $(|\eta| \sim 0)$.

Figures 4.7(a) and (b) show the lepton $p_{\mathrm{T}}$ and $\eta$ distributions for charged leptons at the generator level. The charged leptons are those directly produced from the $W$. In reality, the charged leptons radiate photons at small angles, sequentially losing energy, however most of this energy will be reconstructed in the calorimeters since the photons are emitted with small opening angles. The parton shower should have a minimal effect on the leptons, and this is evident by the agreement of the NLO generators. The AcerMC LO samples tend to produce electrons in the more central region of the detector following the same behavior as the top quark.

\footnotetext{
${ }^{3}$ In particle physics the energy of a particle is represented as a vector with components based on
} 

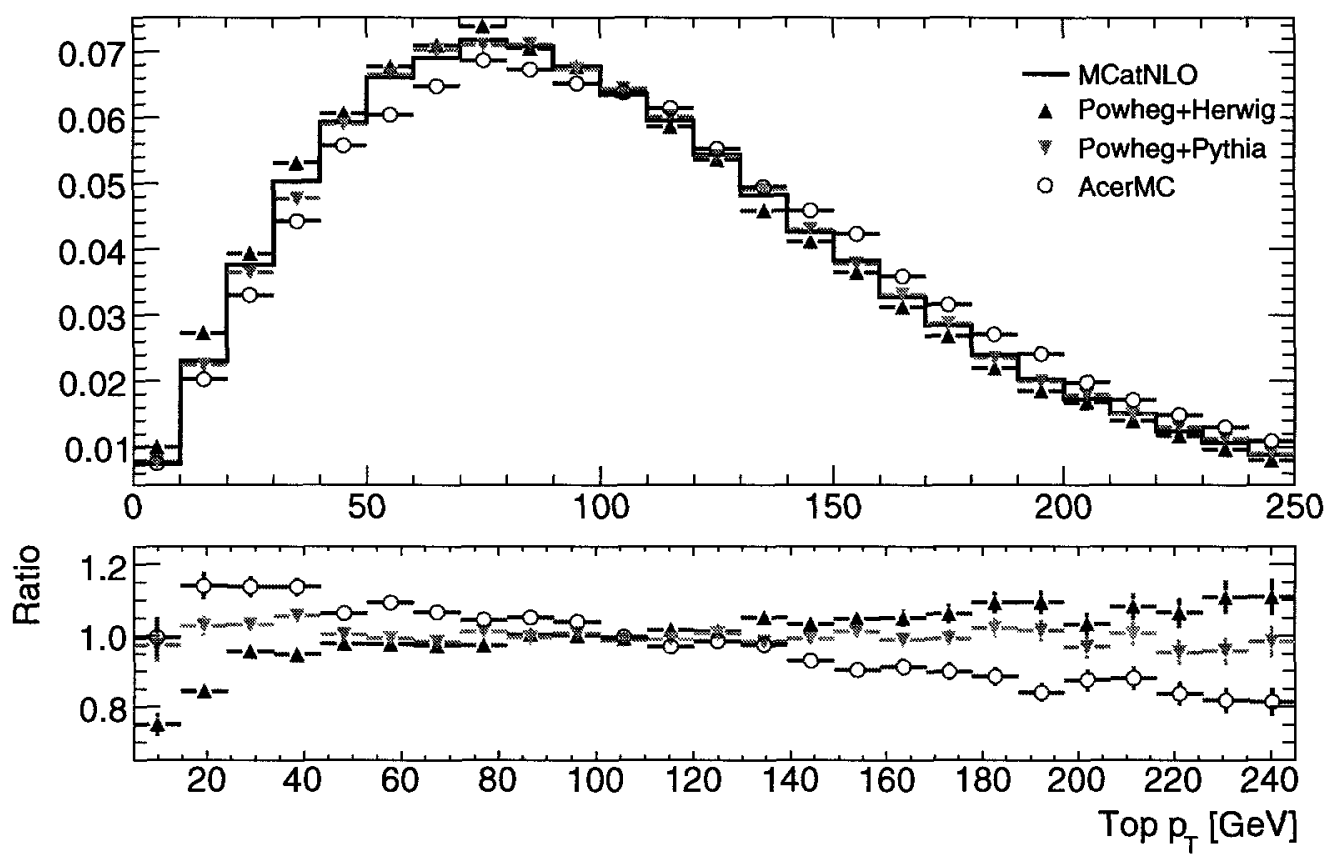

(a)
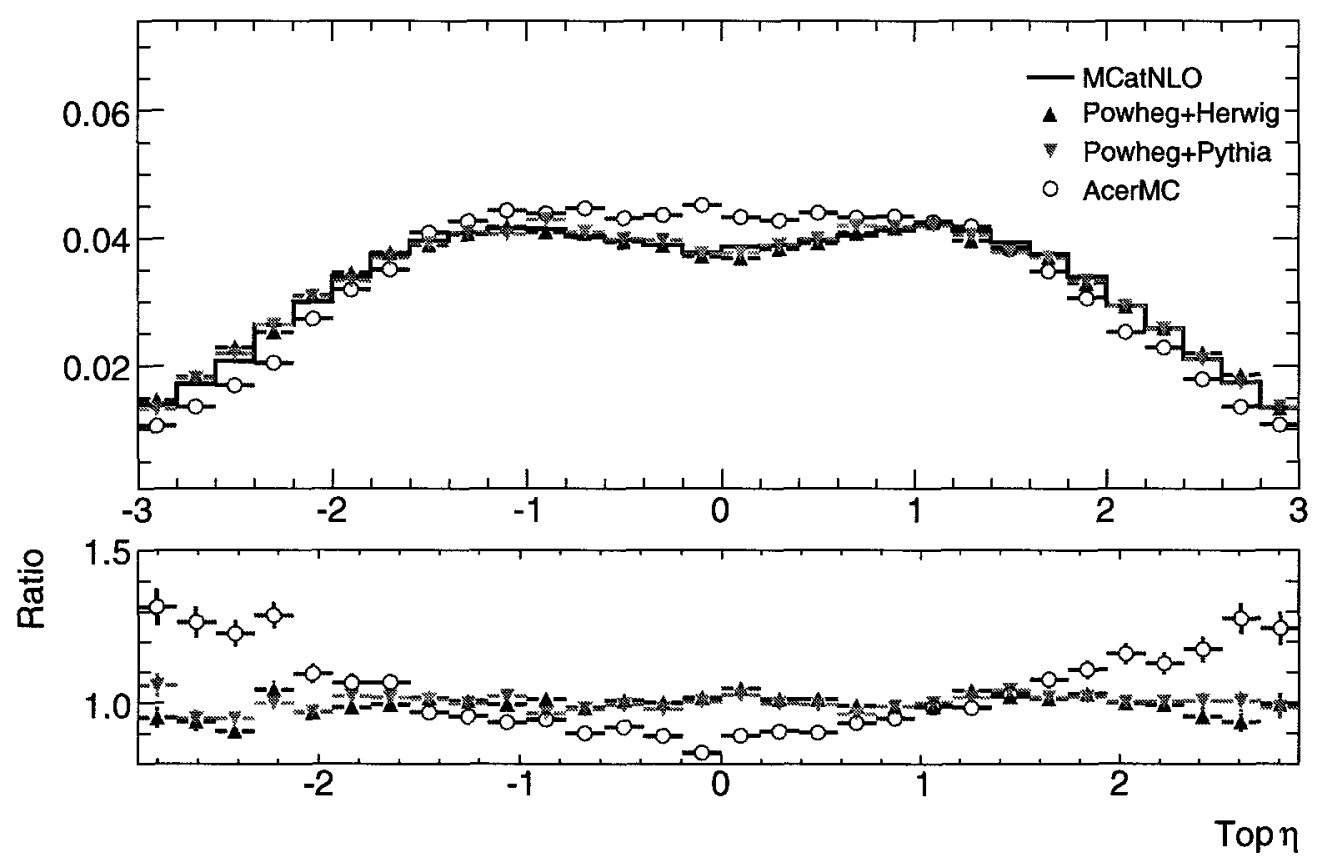

(b)

Figure 4.6: Top quark (a) $p_{\mathrm{T}}$ and (b) $\eta$ distributions for top quarks in the dilepton channel. There are two top quarks per event. 

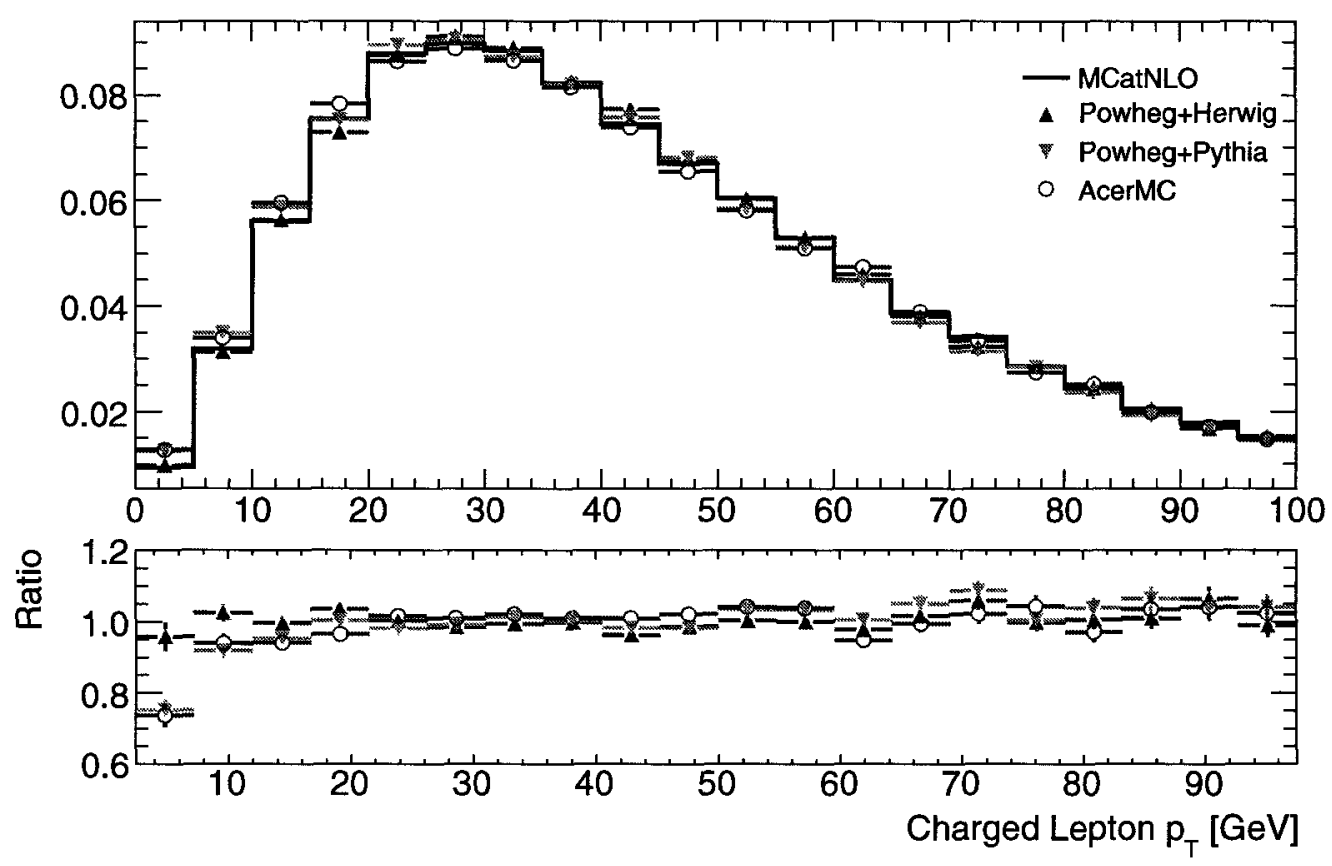

(a)
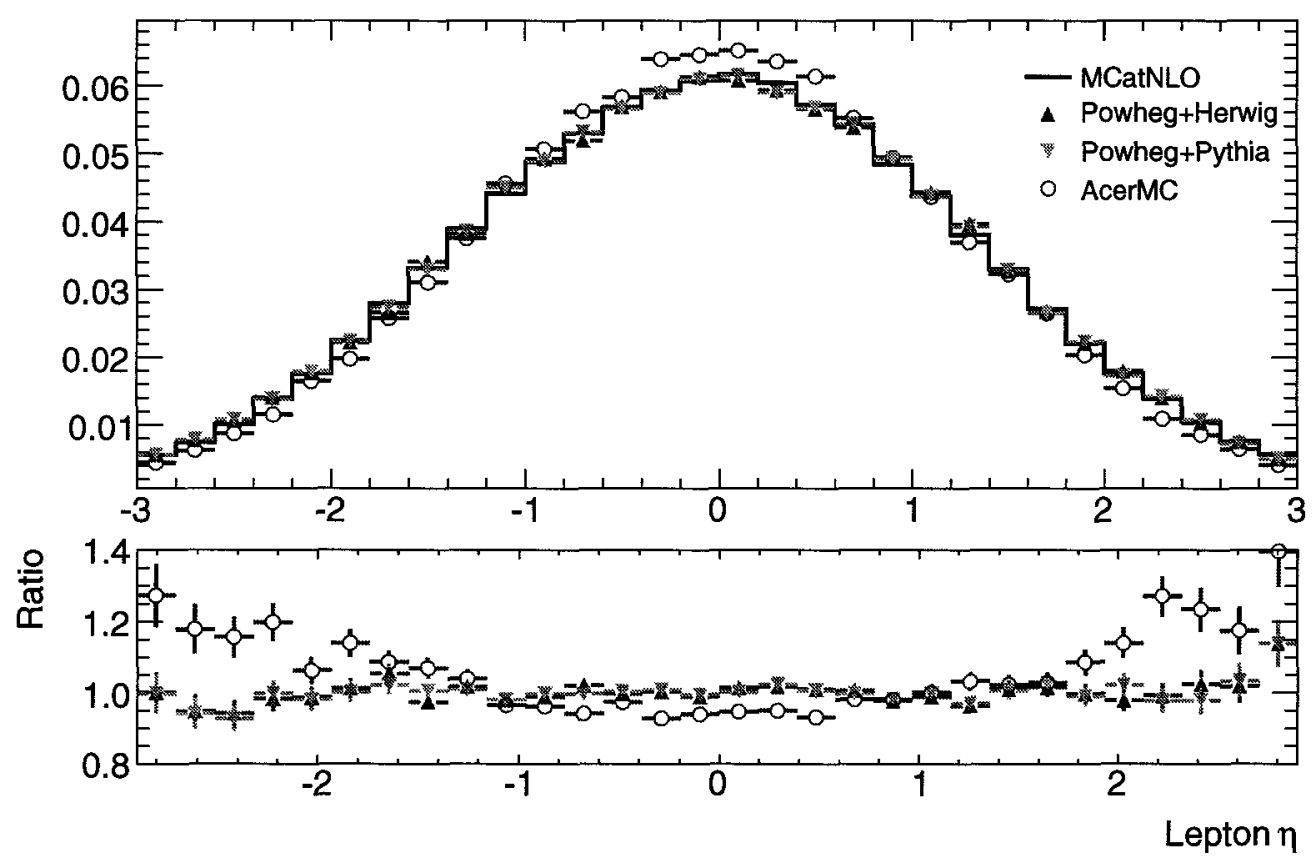

(b)

Figure 4.7: The (a) $p_{T}$ and (b) $\eta$ for charged leptons $(e, \mu, \tau)$ from $W$ in $t \bar{t}$ dilepton events. There are two charged leptons per event. 
Two variables which will be important in identifying $t \bar{t}$ signal events over the background are the summed transverse energy of the neutrinos (often called missing transverse energy as the neutrinos escape the detector without interaction) and the invariant mass of the dileptons, shown at truth level for the four different MC samples in Figures 4.8(a) and (b). There is good agreement between the different generators across a range of energies for both of these variables.

Hard quarks or gluons emitted after the generator subsequently radiate in the parton shower, creating a shower of coloured objects that later combine to form hadrons. These hadrons are grouped together to form a jet, using a jet algorithm called AntiKt4. This jet algorithm will be discussed in Section 4.4.4. The true jet transverse momentum and $\eta$, and jet multiplicity are shown in Figure 4.9. In this case the differences between the parton showers are more evident, as there is a visible difference between the POWHEG samples. POWHEG+HERWIG tends to produce more jets than POWHEG+PYTHIA and MC@NLO. The ratio of the number of jets in the MC@NLO to POWHEG+HERWIG samples appears to be positively correlated with jet multiplicity ranging from a ratio of about 0.6 at low jet multiplicities linearily rising to a ratio of 1.2 in the 12 jet bin. This trend is reversed for the POWHEG+PYTHIA sample with respect to the MC@NLO sample. The POWHEG+HERWIG sample also seems to have a higher multiplicity of low $E_{\mathrm{T}}$ jets.

The results shown above are in agreement with the findings presented in [57] using older MC samples and Athena software.

the total energy, $\theta$, and $\phi$ coordinates. 

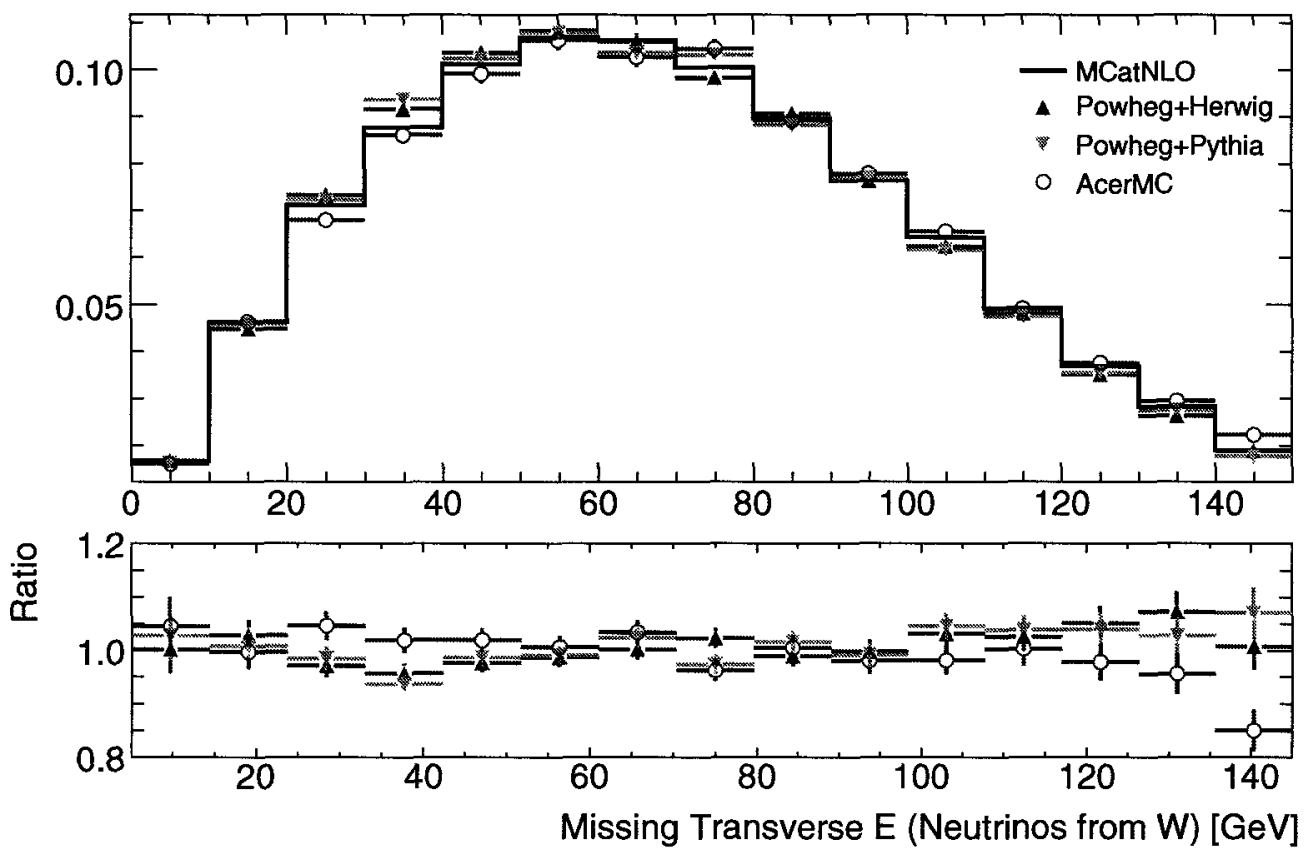

(a)
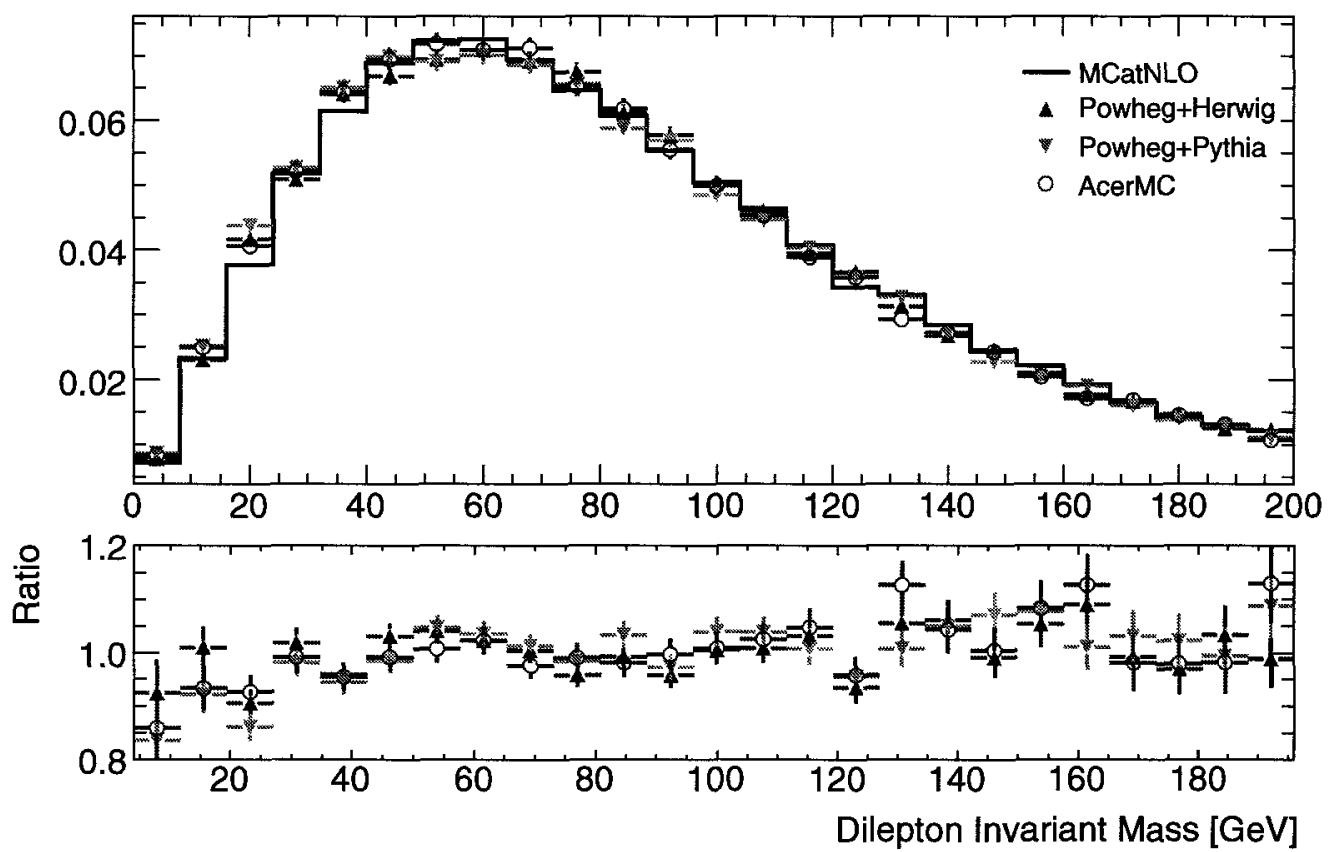

(b)

Figure 4.8: (a) The true total event missing transverse energy from the two neutrinos from the $W$ decays, and (b) the invariant mass of the two charged leptons also coming from $W$ decays. 


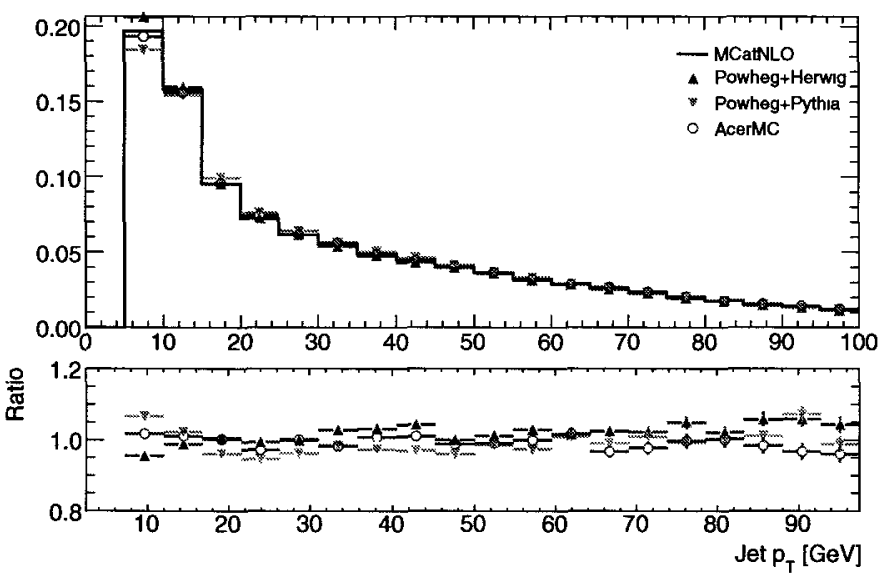

(a)
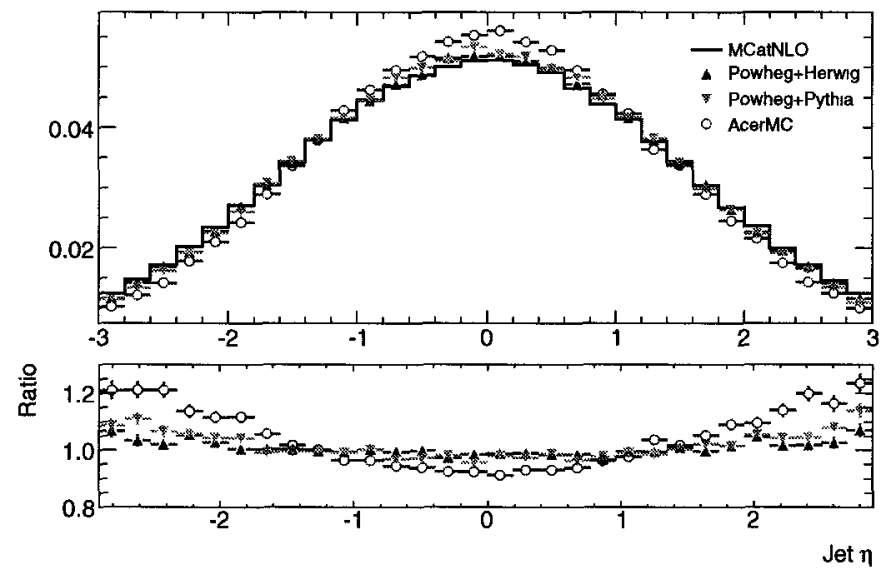

(b)

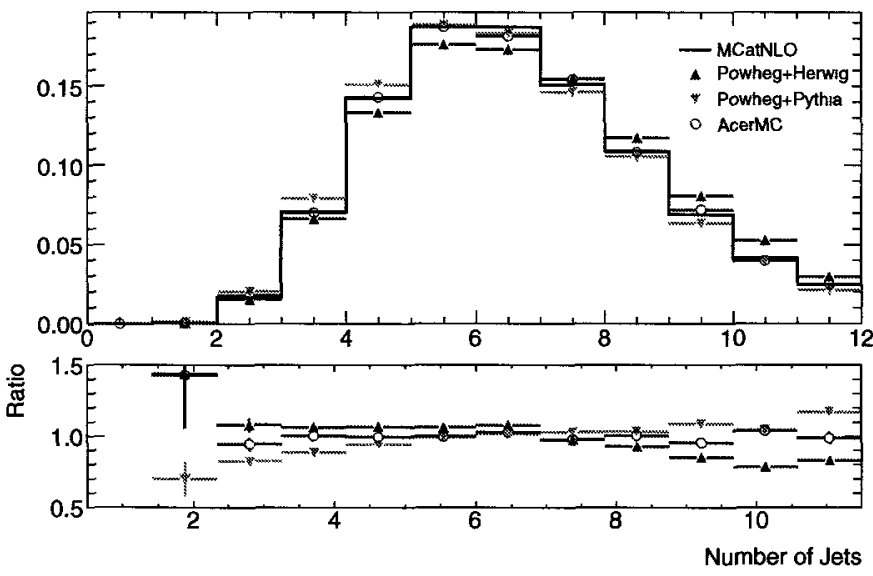

(c)

Figure 4.9: True jet a) transverse momentum, b) $\eta$, and c) number of jets, using the AntiKt4 jet algorithm. 


\subsubsection{Background Samples}

There are several other physics processes that have similar final state characteristics as the signal. The backgrounds considered in this analysis are listed in Table 4.4. Some of these backgrounds do not explicitly contain identical final state objects as the signal, however detector and reconstruction limitations can create fake signatures, such as hadrons being misidentified as electrons in the analysis. Some of these processes are backgrounds because they have very large cross-sections compared to the signal. It is the goal of the analysis described in this thesis to reduce these backgrounds and optimize selections of the physics signal of interest.

The $W \rightarrow l \nu+$ jets process has a large cross-section, and when the number of additional jets produced in the parton shower is two or more this can lead to similar signatures in the detector, especially if one of the jets is mis-reconstructed as a lepton.

It is a similar situation for $Z \rightarrow l l+$ jets, however the best method to suppress this background is by making a cut on the invariant mass of the leptons to ensure it is outside of the $Z$ mass window. A good measurement of the missing transverse energy can also reduce this background.

Although the cross-sections for the diboson backgrounds, $W W, W Z, Z Z$, are small, they have signatures that are very similar to the signal. The contribution from this background can be reduced by discriminating $b$ jets from light jets.

There are backgrounds from other top processes, such as the semi-leptonic $t \bar{t}$ that is different only in the way the $W$ decays.

Additional information about the signal and background samples used, including the ATLAS nomenclature are listed in Appendix A. Only previously observed SM processes are considered in this analysis. 


\begin{tabular}{|l|c|c|c|}
\hline \hline Sample & Matrix Element Generator + Parton Shower & $\sigma[\mathrm{pb}]$ & $k$-factor \\
\hline$W \rightarrow l \nu+$ jets & ALPGEN v2.11+ HERWIG & 26064.9 & 1.22 \\
$Z / \gamma \rightarrow l l+$ jets & ALPGEN v2.11+ HERWIG & 2540.4 & 1.22 \\
$W W \rightarrow l \nu l \nu+$ jets & ALPGEN v2.11+ HERWIG & 3.655 & 1.21 \\
$W Z \rightarrow l l+$ jets & ALPGEN v2.11+ HERWIG & 1.377 & 1.21 \\
$Z Z \rightarrow l l+$ jets & ALPGEN v2.11+ HERWIG & 0.835 & 1.22 \\
$t \bar{t}$ (semi-leptonic) & MC@NLO v3.1+ HERWIG & 64.1 & 1.09 \\
$t \bar{t}$ (fully hadronic) & MC@NLO v3.1+ HERWIG & 64.046 & 1.15 \\
single top $t-$ channel $\rightarrow(e, \mu, \tau)$ & MC@NLO v3.1 + HERWIG & 21.456 & 1 \\
single top $s-$ channel $\rightarrow(e, \mu, \tau)$ & MC@NLO v3.1 + HERWIG & 1.4069 & 1 \\
single top $W t$-channel & MC@NLO v3.1 + HERWIG & 14.581 & 1 \\
QCD & PYTHIA & $9.8 \times 10^{10}$ & - \\
\hline \hline
\end{tabular}

Table 4.4: A list of the background samples considered in this analysis. The cross-sections listed are for $p p$ collisions at $7 \mathrm{TeV}$. The $k$-factor is calculated with respect to NNLO (note there is no $k$ factor calculated for the QCD sample which has a large cross-section and a large theoretical uncertainty) [33].

\subsection{Introduction to the Experimental Calculation of the Cross-section}

MC samples are used to calculate object reconstruction and trigger efficiencies, and event yields for the $t \bar{t}$ dilepton channel and backgrounds. Assuming the SM predictions are correct, at a center of mass energy of $7 \mathrm{TeV}$ the ATLAS detector should collect approximately 3000 dilepton events (including $\tau$ decays) for an integrated luminosity of $200 \mathrm{pb}^{-1}$. However, in the analysis described in the following sections, this is not the final number of events analyzed. Detector acceptance, reconstruction and trigger inefficiencies reduce the final number of events.

\subsection{Reconstructed Object Identification}

The $t \bar{t}$ dilepton channel analysis will require the identification of electrons, muons, jets, and neutrinos in the form of missing energy. There are several algorithms and 
techniques that can be used to identify each particle type, and most rely on the correlation of quantities between the different detector subsystems. The preferred reconstruction method for one physics channel may not be ideal for another, due to different kinematic and event topologies. This section will explore the identification of these reconstructed objects.

The distance between two objects can be measured in $\eta-\phi$ using a geometrical separation defined as:

$$
\Delta R=\sqrt{\Delta \eta^{2}+\Delta \phi^{2}}
$$

where $\Delta \eta=\eta_{1}-\eta_{2}$ and $\Delta \phi=\phi_{1}-\phi_{2}$.

The term 'isolated' is used to refer to an object that is not geometrically (in $\Delta R$ space) close to any other reconstructed object.

Throughout this section the efficiency of a reconstructed object is defined as:

$$
\text { efficiency }=\frac{\text { number of true reconstructed objects }}{\text { number of true objects }}
$$

where the number of true reconstructed objects are those reconstructed objects that fall within some small $\Delta R$ cone of a true object of the same type. The value of $\Delta R$ depends on the object of interest and will be defined for each object in the following sections. The definition of purity is:

$$
\text { purity }=\frac{\text { number of true reconstructed objects }}{\text { number of reconstructed objects }}
$$

There is a trade-off between having a good efficiency and high purity. Typically, these quantities are anticorrelated and the required level of each is dependent on the measurement of interest. Both are important quantities while assessing the validity of a reconstructed object. The statistical error computed on the efficiency and purity 
is discussed in Appendix B.

\subsubsection{Tau Leptons}

In this analysis $\tau$ leptons are not explicitly selected. The $W$ boson has about equal probability to decay to a $\tau$ lepton and its neutrino partner, as either of the other two charged leptons, therefore the dilepton $t \bar{t}$ analysis could include this decay to increase statistics. Because of their large mass, $\tau$ leptons can decay by several modes, including states involving leptons:

$$
\begin{gathered}
\tau \rightarrow \nu_{\tau}+\bar{\nu}_{e}+e \quad(17.4 \%) \\
\tau \rightarrow \nu_{\tau}+\bar{\nu}_{\mu}+\mu \quad(17.8 \%)
\end{gathered}
$$

The numbers in brackets correspond to the branching ratio for these two channels [1]. The $\tau$ lepton can also decay to produce $u, d$, and $s$ quarks resulting in the creation of hadrons like pions or kaons.

The decay of the $\tau$ lepton into two neutrinos plus either a muon or electron will result in kinematics resembling the physics signal of interest. Therefore in this analysis when probing the true event origins these leptonic $\tau$ decays are considered as signal.

\subsubsection{Electrons}

The basic (and ideal) signature of an electron in the ATLAS detector is a track starting in the pixel detector, that moves outward through the SCT and TRT detectors, leading to an isolated region of energy deposition in the electromagnetic calorimeter. Electrons differ from photons by the presence of a charged track in the inner 
detector. Backgrounds to electron identification include hadrons that have a high electromagnetic energy fraction, photon conversions that happen within the inner tracking detectors, and semileptonic decays of heavy flavored quarks.

The following describes the standard electron reconstruction for $|\eta|<2.5$. In the electromagnetic calorimeter, prototype clusters are formed using the sliding window algorithm, which uses a fixed sized window in $\eta-\phi$ space to find clusters. The input to sliding window clustering are towers of size $\Delta \eta \times \Delta \phi=0.025 \times 2 \pi / 256 \mathrm{rad}$. Preclusters combine $5 \times 5$ of these towers, placed to have a local maximum of $E_{\mathrm{T}}$. Preclusters with $E_{\mathrm{T}}$ energy greater than $3 \mathrm{GeV}$ form a seed for the cluster. The electromagnetic sliding window cluster is formed by summing all of the calorimeter channels in a window of $3 \times 7$ units for the barrel $(5 \times 5$ units for the EMEC) with a center defined by the precluster [58]. The electromagnetic sliding window clusters are kept as electron candidates and used for further investigation.

In ATLAS, the group specializing in the identification of electrons have provided several levels of identification cuts for general use, making use of the inner detector and calorimeter quantities. These levels range from a loose to a tight selection, with increasing requirements on the definition of an electron. Many of these selection cuts depend on the electron's $E_{\mathrm{T}}$ and $|\eta|$ of the candidate electron. The definition of each electron selection level follows:

- Loose Electron: The most basic electron reconstruction level. This only uses variables from the calorimeter. A cut is made on the fraction of energy (leakage) deposited in the hadronic calorimeter, requiring it to be a small value (ranging between $1 \%$ and $6.5 \%$ ). Cuts are made using the ratio of energy deposited in the middle layer of the electromagnetic calorimeter in a window of $\eta \times \phi=3 \times 7$ to that deposited in a window of size $7 \times 7$, for isolated electrons this ratio 
should be near one. A cut is also made on the lateral width of the shower in the middle electromagnetic layer.

- Medium Electron: In addition to the Loose requirements, cuts are now made on inner detector quantities and the first layer of the electromagnetic calorimeter. The strips in the first layer of the calorimeter have fine segmentation and are used to study shower shapes. This layer is also used to search for two shower maximum in a small window around the channel with the highest transverse energy; this is done to remove backgrounds from $\pi^{\circ} \rightarrow \gamma \gamma$. The electron is required to have a minimum of one pixel and seven silicon hits on a track that originates from the parton collision point within $1 \mathrm{~mm}$. The track from the inner detector is projected to the calorimeter cluster and must agree within $\Delta \eta<0.1$.

- Medium Isolated Electron: Identical to the Medium Electron selection, but an additional requirement is placed on isolation variables to remove non-isolated electrons in jets. The isolation is defined as the ratio of the transverse energy deposited inside a cone of size $\Delta R=0.2$ and the total transverse energy of the electron, for an isolated electron this should be near one. More information about electron isolation can be found in [59]

- Tight Electron: These electrons pass all of the Medium Electron calorimeter cuts and have additional constraints on tracking variables. The track must match the cluster within a $\Delta \eta<0.005$ and $\Delta \phi<0.02$, and the ratio of the calorimeter energy to the track momentum must be within some range, for the $E_{T}-\eta$ space in this analysis the range is between 0.8 and 2.5 . There is also a minimum number of TRT hits required for a tight electron. In addition, there must be one hit in the vertexing layer ( $b$-layer) to reduce fakes from photons 
converting early within the inner detector.

In this analysis, the identification of electrons will require tracks in the inner detector, therefore a fiducial volume cut is made, requiring electrons to be reconstructed with an $|\eta|<2.47$. It is possible to reconstruct electrons in the forward region as was described in Section 3.9.2, however the leptons in $t \bar{t}$ tend to be produced in the central region (Figure 4.7(b)), and since $t \bar{t}$ events contain many objects, including at least two jets, it has been decided to restrict electron reconstruction to the region spanning the inner detector. The region of $1.37<|\eta|<1.52$ is also excluded to avoid the calorimeter transition region between the barrel and endcap, where there is known to be reduced reconstruction and identification efficiency.

To calculate the efficiency in Equation 4.3 the reconstructed electrons are required to match to a true electron that originates from a $W$ boson or $\tau$ lepton. The matching is based on the geometrical proximity ${ }^{4}$ selecting the closest match in $\Delta R$. Figure 4.10(a) shows the $\Delta R$ between the reconstructed electron and the matched true electron (shown for Loose, Medium, and Tight electrons). The match is considered a success if $\Delta R<0.05$; this cut is superimposed as a dashed line on the figure. As the electron selection gets tighter the outliers of the $\Delta R$ distribution are reduced. Figure 4.10(b) shows the difference in $p_{\mathrm{T}}$ between the reconstructed electrons and the closest true electron for all values of $\Delta R$. A plot of $\Delta R$ versus $\Delta p_{\mathrm{T}}$ for Loose electrons is shown in Figure $4.10 \mathrm{c}$ ), and demonstrates that not all of the matched electrons with large $\Delta p_{\mathrm{T}}$ are removed with the $\Delta R$ cut. Future developments of matching may want to consider the difference between the reconstructed and truth matched $p_{\mathrm{T}}$. The reconstructed electrons tend to have larger reconstructed $p_{\mathrm{T}}$ than the true electrons, this is likely because the true electrons are the final state electrons after all radiation has been emitted, whereas in the reconstruction the calorimeter is

\footnotetext{
${ }^{4}$ Using one of the official ATLAS matching tools for the selection (MCTruthClassifierTool [60]).
} 


\begin{tabular}{|c|c|c|}
\hline Variable & Cut Range & Justification \\
\hline $\begin{array}{l}\qquad|\eta| \\
\qquad|\eta| \\
p_{\mathbf{T}} \\
\text { Isolation } \\
\text { B-layer } \\
\text { Level }\end{array}$ & $\begin{array}{c}<2.47 \\
1.37<|\eta|<1.52 \\
>20 \mathrm{GeV} \\
\text { EtCone } 20<\left[4 \mathrm{GeV}+(0.023) p_{\mathrm{T}}\right] \\
\text { min. one hit } \\
\text { Medium }\end{array}$ & $\begin{array}{l}\text { range of inner detector } \\
\text { remove calorimeter crack } \\
\text { improve rejection of backgrounds } \\
\text { reject contributions from non- } W \text { or } \tau \text { sources } \\
\text { reduce electrons from photon conversions }\end{array}$ \\
\hline Truth matching & $\Delta R<0.05$ & $\begin{array}{l}\text { match reconstructed to true electron } \\
\text { (for efficiency and purity calculation only) }\end{array}$ \\
\hline
\end{tabular}

Table 4.5: Reconstructed electron selection criteria. These cuts are not applied to the truth candidates in the calculation of the efficiency and purity, however true electrons must be produced from a $W$ boson or lepton decay of a $\tau$.

sensitive to collecting the energy of photons which are emitted with a small angle in the electron direction.

The electrons of interest are those coming from $W$ boson decays or leptonic $\tau$ decays. These electrons should be isolated, versus non-isolated electrons that may be included in jets. The sum of the transverse energy in the electromagnetic calorimeter in a cone of size 0.2 (centered on the electron center) minus the electron transverse energy is defined as the variable EtCone20. The isolation requirement is such that:

$$
\text { EtCone20 }<c_{1}+c_{2} p_{\mathrm{T}}
$$

where $c_{1}=4 \mathrm{GeV}$ and $c_{2}=0.023$, and has been optimized by the ATLAS top group [61]. The reconstructed electrons are also required to have $p_{\mathrm{T}}>20 \mathrm{GeV}$, which will later be used to suppress contributions from background processes and fakes. To reduce fake electron signals from photon conversions the candidate electron is required to have at least one hit in the pixel b-layer. Unless otherwise stated, in the rest of this thesis, a reconstructed electron must meet these requirements, which are also listed in Table 4.5. 


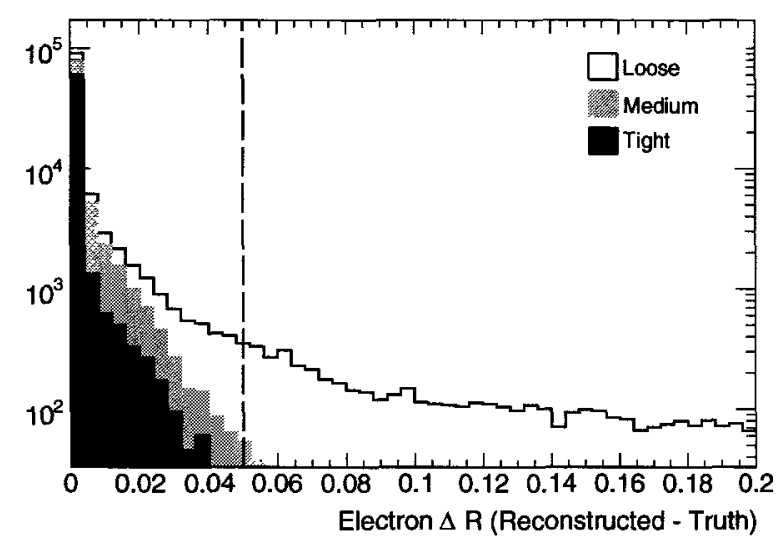

(a)

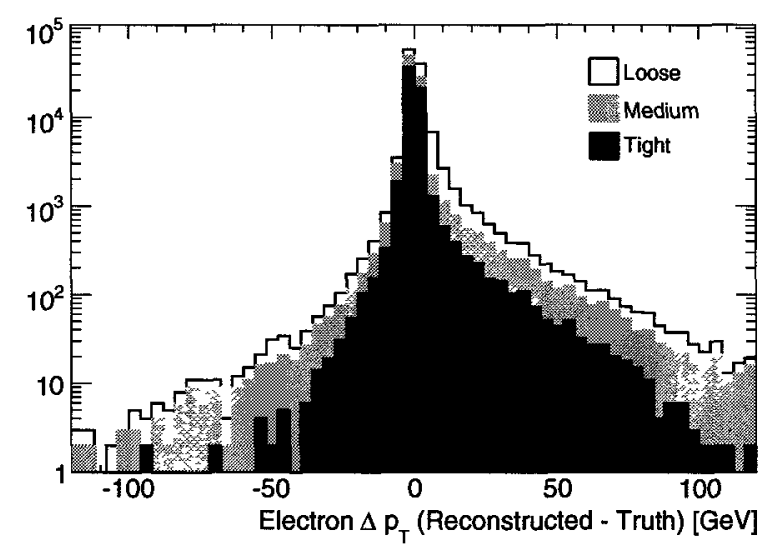

(b)

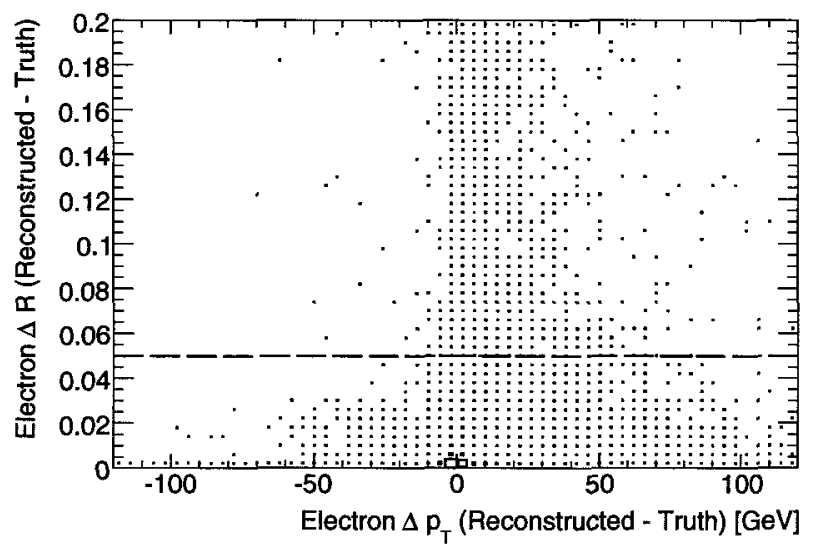

(c)

Figure 4.10: The reconstructed electrons are matched to true electrons for efficiency and purity calculations. These plots show the (a) $\Delta R$ and (b) $\Delta p_{\mathrm{T}}$ between the reconstructed and true electrons. The grey dashed line at $\Delta R=0.05$ shows the cut that is applied in the analysis for calculating the efficiency and purity. Also shown is a plot of (c) $\Delta R$ versus $\Delta p_{\mathrm{T}}$ for Loose electrons. 


\begin{tabular}{|c|c|c|}
\hline \hline Electron Type & Efficiency [\%] & Purity [\%] \\
\hline Loose & $54.3 \pm 0.2$ & $97.8 \pm 0.1$ \\
Medium & $52.8 \pm 0.2$ & $98.4 \pm 0.1$ \\
Medium Isolated & $48.9 \pm 0.2$ & $98.4 \pm 0.1$ \\
Tight & $44.2 \pm 0.2$ & $99.0 \pm 0.1$ \\
\hline
\end{tabular}

Table 4.6: Reconstructed electron efficiency and purity after the selection criteria in Table 4.5 have been applied, but varying the electron classifications, using $t \bar{t}$ dilepton events. These numbers also take into account detector acceptance, which tends to reduce the overall efficiency. The true electrons are only those from $W$ bosons or leptonic $\tau$ decays.

The efficiency and purity (Equations 4.3 and 4.4) were calculated using reconstructed electrons and true electrons originating from a $W$ boson or leptonic $\tau$ decay. The reconstructed electrons must meet the criteria in Table 4.5, except the selection level which is varied. The results for Loose, Medium, Medium Isolated, and Tight electrons are shown in Table 4.6. In going from a Loose electron to a Tight electron the efficiency of reconstructing the signal electrons decreases by about $10 \%$, while the purity only increases by about $1 \%$. In the final analysis electrons of type Medium are used, they offer a good balance between the efficiency and purity for reconstructing signal electrons.

These efficiencies also take into account detector acceptance, as the fiducial volume cuts are applied to the reconstructed electron but not the true electron.

\subsubsection{Muons}

Ideally muons will leave a signature in the inner detector, calorimeter, and muon spectrometer. Basic muon identification involves reconstructing these tracks and ensuring their flight pattern is consistent with originating from the interaction point (as opposed to the downward path of cosmic ray muons). More stringent muon reconstruction demands a match between the track reconstructed in the muon spectrometer 


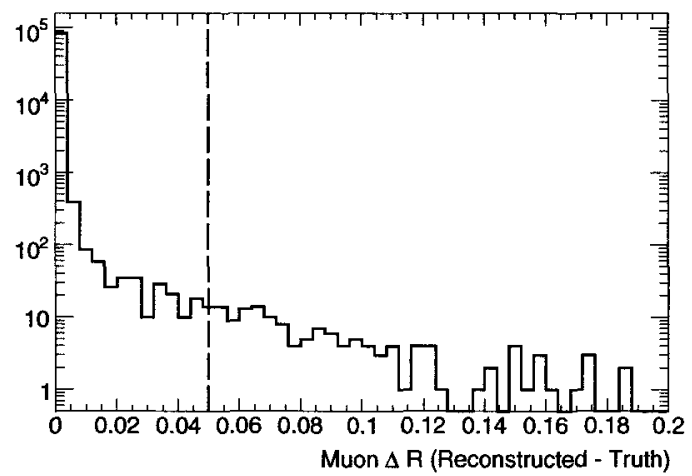

(a)

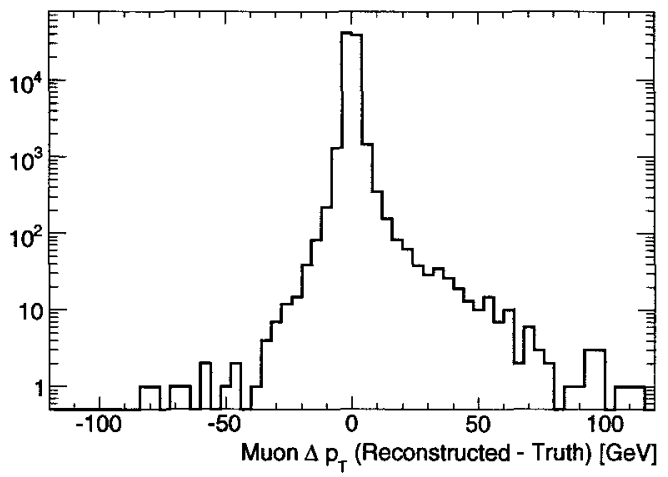

(b)

Figure 4.11: The reconstructed muons are matched to true muons for efficiency and purity calculations. These plots show the a) $\Delta R$ and b) $\Delta p_{\mathrm{T}}$ between the reconstructed and true muons. The grey dashed line at $\Delta R=0.05$ shows the cut that is applied in the analysis for calculating the efficiency and purity.

and inner detector, and requires energy deposition in the calorimeter consistent with a minimum ionizing particle.

The default ATLAS muon reconstruction algorithm uses tracks found in the inner detector and muon spectrometer to make a global fit to the hits in these tracks, taking into account losses and scattering in the calorimeter. For low $p_{\mathrm{T}}$ muons tracks, or regions of insufficient muon spectrometer coverage, tracks are fit using an algorithm which extrapolates from inner detector tracks to the muon spectrometer. The $\Delta R$ and $\Delta p_{\mathrm{T}}$ between reconstructed and true muons are shown in Figure 4.11.

As reconstructed muons are derived from combined inner detector and muon spectrometer measurement, only tracks for which $|\eta|<2.5$ are reconstructed. There are some inefficiencies around $|\eta|=0$ and $|\eta|=1.2$ where the muon spectrometer has reduced coverage. Unless otherwise stated in the text, muons are required to have $p_{\mathrm{T}}>20 \mathrm{GeV}$. A requirement is also placed on the isolation of the muon to reduce the number of reconstructed non-isolated muons coming from jets. The calorimeter isolation variable (EtCone30) is the difference in $E_{\mathrm{T}}$ between energy contained with a 


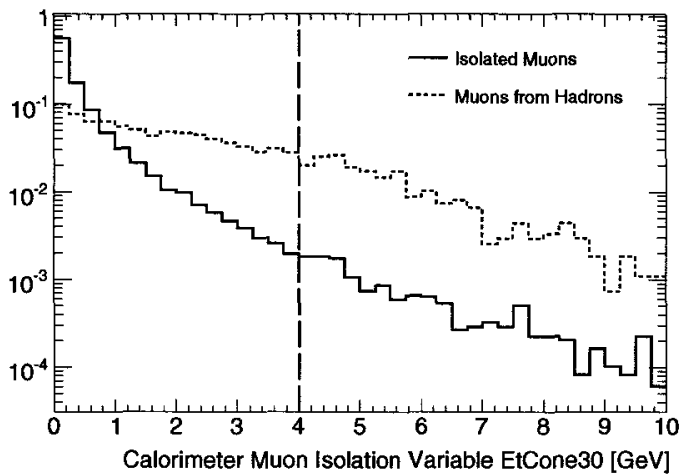

(a)

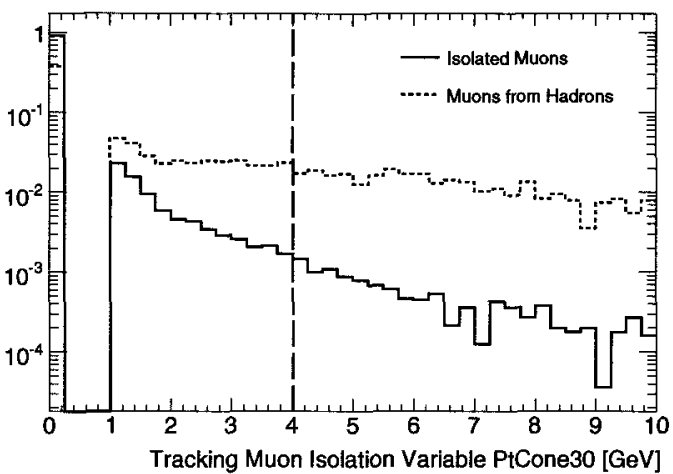

(b)

Figure 4.12: Calorimeter isolation $E_{\mathrm{T}}$ shown to the left, and track isolation in $p_{\mathrm{T}}$ shown to the right for isolated muons coming from $W$ bosons or $\tau$ leptons and non-isolated muons from hadron decays in $t \bar{t}$ dilepton events. In the analysis a good reconstructed muon is required to have calorimeter and track isolation less than $4 \mathrm{GeV}$ (shown by the vertical dashed line).

cone of size $\Delta R=0.3$ and $\Delta R=0.15$ centered on the muon. There is a track isolation criteria (PtCone30) in which the $p_{\mathrm{T}}$ of all the tracks within $\Delta R=0.3$ centered on the muon track (excluding the muon track) are summed together. Both the calorimeter and track isolation sum variables are required to be less than $4 \mathrm{GeV}$. Distribution of the calorimeter and track isolation are shown in Figure 4.12 for isolated and nonisolated muons in $t \bar{t}$ dilepton events. The muons in those figures were required to have an $|\eta|<2.5$ and were matched to a true muon with $\Delta R<0.05$ (a $p_{\mathrm{T}}$ cut was not applied as many of the non-isolated muons have low momentum).

Good reconstructed muons are required to fulfill the criteria listed in Table 4.7. The muon reconstruction efficiency was found to be $62.7 \pm 0.2 \%$, with a purity of $100 \%$. 


\begin{tabular}{|c|c|l|}
\hline \hline Variable & Cut Range & Justification \\
\hline $\begin{array}{c}|\eta| \\
p_{\mathrm{T}} \\
\text { Isolation }\end{array}$ & $\begin{array}{c}<2.5 \\
20 \mathrm{GeV}\end{array}$ & $\begin{array}{l}\text { limit of inner detector, require track match } \\
\text { improve rejection of backgrounds } \\
\text { reduce fakes (from jets) }\end{array}$ \\
\hline Truth matching & $\Delta R<0.05$ & $\begin{array}{l}\text { match reconstructed to true muon } \\
\text { (for efficiency and purity calculation only) }\end{array}$ \\
\hline
\end{tabular}

Table 4.7: Reconstructed muon selection criteria. These cuts are not applied to the truth candidates in the calculation of the efficiency and purity, however true muons must be produced from a $W$ boson or lepton decay of a $\tau$.

\subsubsection{Jets}

Jets are compound objects composed of many different types of particles, including charged and neutral hadrons and leptons. The particles in a jet are the final state products from the fragmentation of a coloured object. The net kinematical properties of a jet reflect the properties of the initial gluon or quark. The characteristics of a reconstructed jet depend on how these individual particles are collected together, therefore the definition of a reconstructed jet is defined by the algorithm that creates it. In general, the grouping of particles into jets is done by their geometric proximity to each other. In ATLAS there are several steps taken to form a jet:

Define input objects: These are the basic constituents that will be used to find jets. These are either jet tower clusters or topological clusters. Jet towers are formed from a $\Delta \eta \times \Delta \phi=0.1 \times 0.1$ grid, summing all electromagnetic and hadronic calorimeter channels that fall within this grid. Topological clusters are formed based on the significance of the energy in a given channel and the proximity to other channels with significant energy deposition (a full description of the topological clustering algorithm was presented in Section 3.7.2).

Jet finding: There are two options used in this analysis to search for jets called the 
cone algorithm and the (anti-) $k_{\mathrm{T}}$ algorithm.

The cone algorithm uses all of the input objects above some seed threshold $\left(E_{\mathrm{T}}>1 \mathrm{GeV}\right)$, and groups them into a cone of size $R_{\text {cone }}=\sqrt{\Delta \eta^{2}+\Delta \phi^{2}}$ centered such that the sum of the transverse energy of all objects within the cone is maximized. Several cones can be made, making what are called protojets, and each can use one or more of the input objects. If cones overlap then the decision to split or merge the protojets is based on the fraction of shared energy to the energy of the softer protojet. If the fraction is large $(>0.75)$ then the two protojets are merged into a new protojet, otherwise they are split. The process is repeated over all protojets until there are no overlap, at which point all remaining protojets become jets. In ATLAS there are two cone sizes available, Cone04 with $R_{\text {cone }}=0.4$ and Cone07 with $R_{\text {cone }}=0.7$.

The (anti-) $k_{\mathrm{T}}$ algorithm searches for jets using the following routine. Note that throughout, the constant $c$ is defined as:

$$
c= \begin{cases}+1 & \text { for the } k_{\mathrm{T}} \text { algorithm }[62,63] \\ -1 & \text { for the anti- } k_{\mathrm{T}} \text { algorithm [64] }\end{cases}
$$

(1) For every possible combination of objects $i$ and $\jmath$, a variable $d_{\imath \jmath}$ is defined:

$$
d_{\imath \jmath}=\min \left(p_{\mathrm{T}, \imath}^{2 c}, p_{\mathrm{T}, \jmath}^{2 c}\right) \frac{\Delta R_{\imath \jmath}^{2}}{R^{2}}
$$

where $p_{\mathrm{T}, 2}$ is the transverse momentum of the $i$ th object, and $\Delta R_{\imath \jmath}=\sqrt{\left(y_{\imath}-y_{\jmath}\right)^{2}+\left(\phi_{\imath}-\phi_{\jmath}\right)^{2}}$ using the rapidity and polar angle of the two objects. The variable $R$ is a parameter of the algorithm, and in ATLAS can have the values of 0.4 or 0.6 with the respective naming convention of (Anti)Kt4 or (Anti)Kt6.

(2) For each object define:

$$
d_{\imath \imath}=p_{\mathrm{T}, \imath}^{2 c} .
$$


(3) If the minimum value of all $d_{\imath \imath}$ and $d_{\imath \jmath}$ is a $d_{\imath \jmath}$ then objects $i$ and $j$ are merged together to form a new object. If the minimum is a $d_{\imath \imath}$ then the $i$ th object is defined as a jet and removed from the list of objects.

(4) The routine is repeated until there are no more objects in the list.

Jet calibration: The jets defined above have their energy and momentum calculated at the electromagnetic scale. Additional calibrations are then applied to account for differences of the calorimeter response to hadronic and electromagnetic showers. In ATLAS the default jet calibration scheme recalibrates the calorimeter channels in the jet using the $H 1$ style weights $\omega\left(\rho_{2}, \vec{X}_{2}\right)$ [65]. These weights $\omega\left(\rho_{2}, \vec{X}_{2}\right)$ depend on the energy density of the channel $\rho_{\imath}=E_{\imath} / V_{\imath}$ and the position of the channel $\vec{X}_{\imath}$. The weights were extracted by minimizing the resolution with respect to the true reconstructed jet energy (using Cone07) on a dijet MC sample. Since the definition of a jet depends on the jet finding algorithm, additional corrections are made to jets found by algorithms other than Cone07. Alternative hadronic calibration procedures are being explored in ATLAS that make use of the shower shape variables and apply corrections at the cluster level.

Jet selection: The jet multiplicity and $p_{\mathrm{T}}$ of reconstructed jets in $t \bar{t}$ dilepton events are shown in Table 4.8, before any cuts are applied, for six different combinations of input objects and jet finding algorithm. The Cone07 has a wide cone, so this jet reconstruction has fewer jets per event, but each jet tends to have slightly higher $p_{\mathrm{T}}$ than the other algorithms. Currently, the default algorithm for use in ATLAS is the AntiKt4Topo as it is theoretically both infrared and collinear safe, results in jets with nice cone-like shapes, and provides the best reconstruction efficiency. 


\begin{tabular}{|l|c|c|}
\hline \hline Jet Reconstruction & Avg. Jet Multiplicity & Avg. Jet $p_{\mathrm{T}}[\mathrm{GeV}]$ \\
\hline AntiKt4H1Topo & 6.2 & 36.7 \\
AntiKt4H1Tower & 6.2 & 36.7 \\
Cone4H1Topo & 6.0 & 38.2 \\
Cone4H1Tower & 6.2 & 42.9 \\
Cone7H1Tower & 4.5 & 51.0 \\
AntiKt6H1Topo & 6.7 & 37.2 \\
\hline \hline
\end{tabular}

Table 4.8: The average number of reconstructed jets per event and average $p_{\mathrm{T}}$ of each, for six different jet reconstruction methods.

To compute the efficiency of jet reconstruction, the Cone04 and AntiKt4 jet finding algorithms were also applied to generated particles, using as input objects to the jet finder all final state stable neutral and charged particles with $|\eta|<5$. The $\Delta R$, and $\Delta p_{\mathrm{T}}$ of the closest matched jet, are shown in Figure 4.13 for the AntiKt jets using topological clusters and Cone04 tower jets, both calibrated with $H 1$ weights. Both algorithms show similar behavior for the $\Delta R$ and $\Delta p_{\mathrm{T}}$ distributions. The $\Delta R$ distributions are wider than for electrons, reflecting the more complex definition of a jet object. From the tails of the $\Delta p_{\mathrm{T}}$ of the closest matched jet shown in Figure 4.13(b), it appears the jet reconstruction algorithm underestimates the true jet energy. A successful match between a reconstructed jet and truth jet occurs if $\Delta R<0.1$.

The final selection for jets is largely analysis dependent, for this analysis $t \bar{t}$ events are required to have a minimum of two jets (at least two are produced as $b$ jets, and possibly there are more jets from higher order processes), and each jet must have $p_{\mathrm{T}}>20 \mathrm{GeV}$ and be within $|\eta|<2.5$. These cuts are listed in Table 4.9.

The reconstruction efficiency of the AntiKt4 (Topo and Tower) algorithm with respect to true particles reconstructed with the AntiKt4, and the same for the Cone4 algorithm, are shown in Table 4.10. The reconstructed jets are expected to satisfy the $\eta$ and $p_{\mathrm{T}}$ cuts in Table 4.9. A true reconstructed jet must also satisfy the truth 


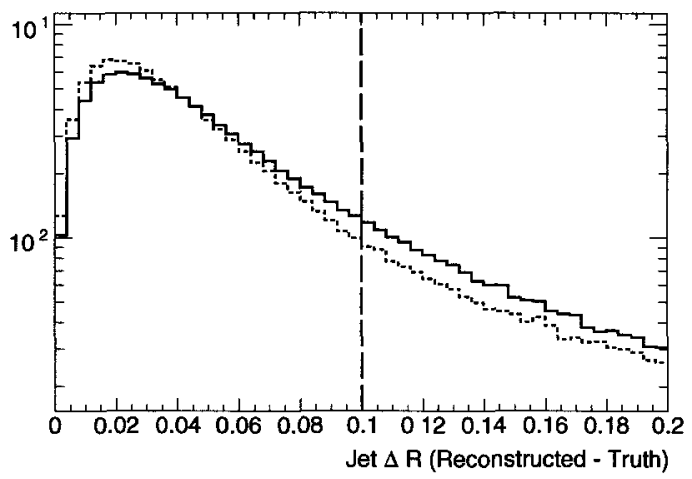

(a)

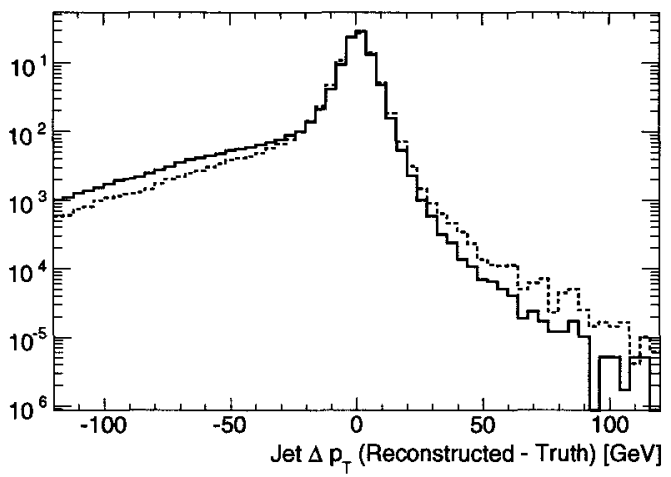

(b)

Figure 4.13: a) $\Delta R$ and b) $\Delta p_{\mathrm{T}}$ between reconstructed jets and true jets, shown for AntiKt4H1Topo jets with respect to AntiKt4 truth jets (solid line) and Cone4H1Tower jets and Cone4 truth jets (dashed line).

\begin{tabular}{|c|c|l|}
\hline \hline Variable & Cut Range & Justification \\
\hline$|\eta|$ & $\begin{array}{c}<.5 \\
p_{\mathrm{T}}\end{array}$ & $\begin{array}{l}\text { range of inner detector (for future } b \text {-jet tagging studies) } \\
\text { improve rejection of backgrounds }\end{array}$ \\
\hline Truth matching & $\Delta R<0.1$ & $\begin{array}{l}\text { match reconstructed to true jet of same algorithm } \\
\text { (for efficiency and purity calculation only) }\end{array}$ \\
\hline \hline
\end{tabular}

Table 4.9: Reconstructed jet selection criteria. These cuts are not applied to the truth candidates in the calculation of the efficiency and purity. In addition to these cuts there is an overlap removal performed between a jet and electron if a jet is within a cone of $\Delta R=0.2$ of a reconstructed electron (only one jet is removed per event), overlap removal is discussed in Section 4.4.5.

matching criteria. The AntiKt4 and Cone4 algorithms, with inputs of either towers or topological clusters behave similarly for reconstructing jets in $t \bar{t}$ dilepton events, with the AntiKt4 algorithm having a slightly better reconstructed efficiency and purity.

\subsubsection{Object Overlap Removal}

In the ATLAS reconstruction software if a region of the detector has a signature of both an electron and jet and/or muon all of these objects will be reconstructed. In certain situations this may lead to fake signatures. An overlap removal between 


\begin{tabular}{|l|c|c|}
\hline \hline Jet Reconstruction & Efficiency [\%] & Purity [\%] \\
\hline AntiKt4H1+Topo & $43.2 \pm 0.1$ & $93.1 \pm 0.1$ \\
AntiKt4H1+Tower & $42.9 \pm 0.1$ & $93.6 \pm 0.1$ \\
Cone4H1+Topo & $41.3 \pm 0.1$ & $90.5 \pm 0.1$ \\
Cone4H1+Tower & $41.2 \pm 0.1$ & $91.4 \pm 0.1$ \\
\hline \hline
\end{tabular}

Table 4.10: Jet reconstruction efficiency and purity for the AntiKt4 and Cone4 algorithms taking as inputs the topological cluster (Topo) and towers. The reconstructed jets must meet the criteria listed in Table 4.9 .

reconstructed objects has been employed throughout this thesis following this method:

1. The proximity of each reconstructed electron was compared to each reconstructed jet. For each electron, if the closest jet was within $\Delta R<0.2$ then that jet was removed from the list of good jets. The electrons considered were those that passed the criteria listed in Table 4.5 (except truth matching).

2. For all of the jets that did not overlap with any electrons and satisfied the good jet reconstruction requirements in Table 4.9 (except the truth matching cut), the distance to each muon was calculated. If the muon and jet overlapped with $\Delta R<0.4$ the muon was considered to be part of the jet and removed from the analysis.

The order of electrons, jets, and then muons was followed based on the underlying physics that might contribute to the overlaps and knowledge about the ability to identify these objects in the detector. It is assumed that small amount of energy deposited in the calorimeter by a single isolated muon would not be sufficient to create a reconstructed jet. 


\subsubsection{Missing Transverse Energy}

The detection of non-interacting particles, such as neutrinos, are made by summing all of the visible energy in the detector and using this to infer the amount of energy carried away by them. When the partons collide it is assumed that they have zero net energy in the transverse plane, however it is unknown what fraction of the proton's energy is carried by the colliding partons, hence it is impossible to know the net energy along the $z$ direction. The transverse missing energy is calculated from the net sum of energy in the $x$ and $y$ planes:

$$
\begin{aligned}
& E_{\mathrm{T}}^{\mathrm{miss}}=\sqrt{\left(E_{x}^{\mathrm{miss}}\right)^{2}+\left(E_{y}^{\mathrm{miss}}\right)^{2}} \\
& E_{x}^{\mathrm{miss}}=-\sum E_{x}, \quad E_{y}^{\mathrm{miss}}=-\sum E_{y}
\end{aligned}
$$

where the sum is over all objects contributing to the calculation in the event. The choice and treatment of these objects is discussed in the following paragraphs. In $t \bar{t}$ dilepton events there are a minimum of two neutrinos per event, so it will not be possible to calculate the energy of the individual neutrinos, only the sum of their energies in the transverse plane.

In this analysis $E_{\mathrm{T}}^{\text {miss }}$ has been calculated using the sum over $E_{x}$ and $E_{y}$ with contributions from three terms:

$$
\sum E_{x, y}=E_{x, y}^{\text {calo }}+E_{x, y}^{\text {muon }}+E_{x, y}^{\text {cryo }}
$$

The first term calculates the contribution of particle energies collected in the channels of the calorimeter. All of the high $p_{\mathrm{T}}$ objects are stored in a list taking the order: $e$, $\gamma$, hadronically decaying $\tau$ leptons, jets, $\mu$. For each object in the list, starting with the first object, the calorimeter channels used to reconstruct the object are identified 
and calibrated to the appropriate scale for that object (for example the application of $H 1$ weights to jet objects). If a channel belongs to more than one object it is only associated to the first object in the list to avoid double counting of energy. If a channel in the calorimeter is not associated to any high $p_{\mathrm{T}}$ object it is still included in the summation, and is calibrated using the $H 1$ weights. $^{5}$

The second term in Equation 4.6, $E_{x, y}^{\text {muon }}$ calculates the amount of energy of each muon. Muons tend to deposit a small amount of energy in the calorimeter so summing the channels in the calorimeter is not sufficient. This energy will already be included in the term $E_{x, y}^{\text {calo }}$. The remaining muon energy not deposited in the calorimeter is calculated using the momentum measurement in the muon spectrometer.

The final term, $E_{x, y}^{\text {cryo }}$, calculates the amount of energy lost in the cryostat. For the barrel this is energy lost between the last electromagnetic layer and the first hadronic layer, and for the endcap it is the energy lost upstream in the cryostat. This correction is in the form of a weight extracted alongside the $H 1$ weights using $\mathrm{MC}$ simulations.

The performance of $E_{\mathrm{T}}^{\text {miss }}$ is made with respect to the true missing transverse energy coming from all non-interacting final stable particles. The difference of the reconstructed and true $E_{\mathrm{T}}^{\mathrm{miss}}$ is shown in Figure 4.14(a). The mean of the distribution is $-0.87 \mathrm{GeV}$, indicating the reconstructed $E_{\mathbf{T}}^{\text {miss }}$ slightly overestimates the value. The linearity, defined as:

$$
\text { linearity }=\frac{\text { true } E_{\mathrm{T}}^{\text {miss }}-\text { reconstructed } E_{\mathrm{T}}^{\text {miss }}}{\operatorname{true} E_{\mathrm{T}}^{\text {miss }}}
$$

is shown in Figure 4.14(b) and shows the reconstructed $E_{\mathrm{T}}^{\text {miss }}$ is about $4 \%$ higher than the truth when $E_{\mathrm{T}}^{\text {miss }}>50 \mathrm{GeV}$, otherwise the reconstruction underestimates $E_{\mathrm{T}}^{\text {miss }}$.

\footnotetext{
${ }^{5}$ In ATLAS terminology this algorithm is the refined missing transverse energy calculation.
} 


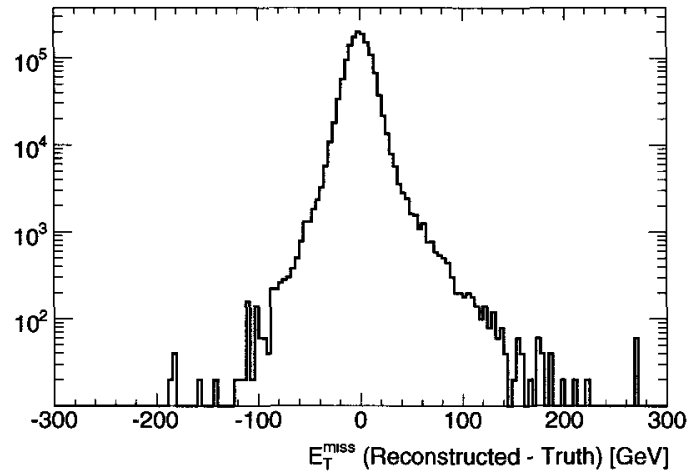

(a)

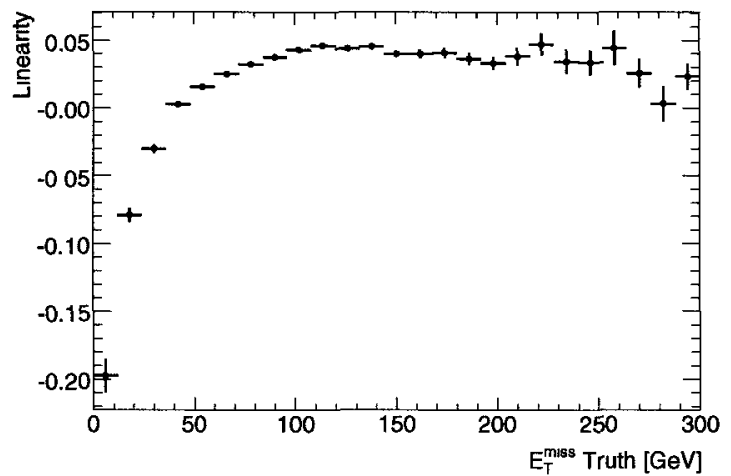

(b)

Figure 4.14: (a) Reconstructed missing transverse energy difference with respect to the truth, (b) and linearity as defined in Equation 4.7.

Issues with $E_{\mathrm{T}}^{\text {miss }}$ potentially arise due to the definition of objects, such as muons. It has already been shown that the efficiency to reconstruct electrons, muons, and jets is below one. For $E_{T}^{\text {miss }}$, muons are only defined with $|\eta|<2.7$, so muons outside of this $\eta$ range contribute to false $E_{\mathrm{T}}^{\mathrm{miss}}$.

The fraction of events that pass as a function of $E_{\mathrm{T}}^{\mathrm{miss}}$ cut is shown in Figure 4.15 for both the reconstructed and true $E_{\mathrm{T}}^{\text {miss }}$. With a $E_{\mathrm{T}}^{\text {miss }}$ cut of approximately $60 \mathrm{GeV}$ about $50 \%$ of the true dilepton events are cut. The choice of this cut depends on the balance between loss of signal and rejection of backgrounds.

\subsubsection{Overlap Removal and Missing Transverse Energy}

The $E_{\mathrm{T}}^{\text {miss }}$ was not recalculated after the overlap removal algorithm was applied. The overlap removal method could be improved by recalculating the missing transverse energy using only true objects defined after the overlap removal procedure. 


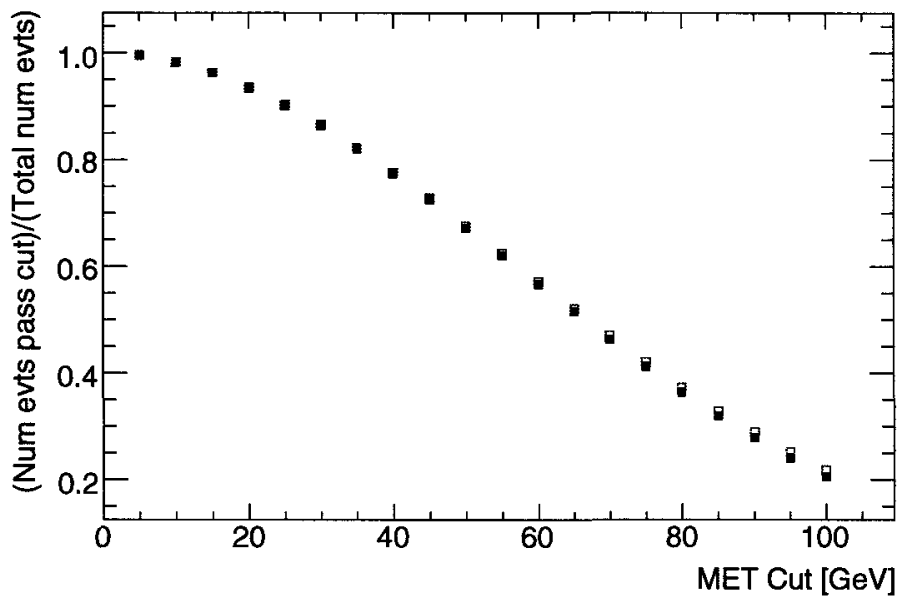

Figure 4.15: Event efficiency as a function of transverse missing energy cut, for reconstructed $E_{\mathrm{T}}^{\text {miss }}$ (black squares) and true $E_{\mathrm{T}}^{\text {miss }}$ (open squares).

\subsection{The ATLAS Trigger}

The LHC is designed to collide protons together in the ATLAS detector every $25 \mathrm{~ns}$, a rate of 40 million collisions per second. The job of the trigger is to decide which events to accept and write to tape for further analysis. In ATLAS this is achieved through three trigger levels, referred to as level 1 (L1), level 2 (L2), and event filter (EF). As one goes through the sequence of triggers from L1 to EF the event rate decreases.

The L1 trigger is used to reduce the event rate from $40 \mathrm{MHz}$ to approximately $75 \mathrm{kHz}$, with a processing time of $2.5 \mu \mathrm{s}$. This is a hardware based trigger, using information from coarse regions of the calorimeter and muon systems. Once an event passes the L1 trigger it is passed to the high level trigger (HLT). The HLT is formed from the L2 and EF triggers, and makes use of the full detector granularity in its hypothesis testing. At L2 the maximum event rate is reduced to $3.5 \mathrm{kHz}$ (with a processing time of $40 \mathrm{~ms}$ ), and in the EF to $200 \mathrm{~Hz}$ (with a processing time of $4 \mathrm{~s}$ ). The term online refers to the trigger decisions being made promptly as the data is 
being read out of the detector. The term offline refers to software that is used after the trigger decisions, and in principle has no time limit, and therefore can apply more complex calibrations and corrections.

The unavoidable first cut to any analysis is that of the trigger. It is important for each analysis to select a trigger that provides the highest efficiency for capturing the signal events. It is also important to understand the efficiencies of these triggers. Since the trigger is required to make split second decisions it is not possible to use complicated calibrations. The approximations used in the calibrations must be understood to minimize any biases introduced, and then any residual biases must be accounted for in the analysis. The trigger efficiency is one key number in any cross-section measurement, it can reduce the observed number of events with respect to the expected number, and can potentially vary with the cuts being applied later in the analysis.

There are many triggers available for use. The trigger menu used in ATLAS is described below in Section 4.5.2. Ultimately, the choice of trigger is channel dependent and a trigger study is required for each analysis. There are triggers defined by the presence of single objects, such as triggers that will pass if one electron with some minimum $E_{\mathrm{T}}$ is detected. Alternatively, there are triggers that make use of a combination of objects, such as the presence of one jet and one electron. The $t \bar{t}$ signal is complex, containing leptons, missing transverse energy, and $b$-jets. For the specific case of the $t \bar{t}$ dilepton channel there are always at least two leptons; in a hadron environment triggering on leptons is the ideal choice. This analysis will explore the single lepton and dilepton triggers (where leptons $=e, \mu$ ). 


\subsubsection{Naming Convention}

In ATLAS the names given to the triggers follow a convention that encodes information about the trigger. The first grouping of letters signifies the trigger level by either L1, L2, or EF. Following the level is a list of the required physics objects for that trigger. This could be an 'e' for a single electron or ' $2 \mathrm{mu}$ ' for two muons. For the L1 trigger the physics objects are in capitals (ex. 'MU') and for the HLT they are in lower case (ex. 'mu'). Associated to each physics object is the minimum $E_{\mathrm{T}}$ or $p_{\mathrm{T}}$ that must be satisfied by the physics object. Sometimes the object must meet some isolation criteria (such that the object is not geometrically near another object). In this case the $E_{\mathrm{T}}$ threshold will be followed by an 'I' (L1) or ' $\mathrm{i}$ ' (HLT). Some triggers have the words 'loose', 'medium, or 'tight' to represent some additional selection criteria on the object, such as those discussed in Section 4.4.2 for electron reconstruction. An example of a trigger name is L2_mu6, which is a L2 trigger that requires one muon with a minimum $p_{\mathrm{T}}$ of $6 \mathrm{GeV}$ to pass.

\subsubsection{Trigger Menu and Trigger Rates}

A trigger menu is a collection of different triggers that are enabled and used to select events to write out for further analysis. The electron and muon triggers available and used in this thesis are listed in Table 4.11. The trigger chain is the name associated to the logical sequence of input triggers that run through the three trigger levels.

There is a maximum total allowed rate at each trigger level, so predictions are made based on minimum bias $\mathrm{MC}$ samples of the contribution to the total rate from each possible trigger. The rate for each trigger depends on the instantaneous luminosity of the LHC, which determines the amount of pileup activity in the colliding proton bunches. Luminosity will be discussed in greater detail in Section 4.7.3. If the 


\begin{tabular}{|c|c|c|c|}
\hline \hline Trigger Chain & L1 & L2 & EF \\
\hline e5_medium & L1_EM5 & L2_e5_medium & EF_e5_medium \\
2e5_medium & L1_2EM3 & L2_2e5_medium & EF_2e5_medium \\
e10_medium & L1_EM5 & L2_e10_medium & EF_e10_medium \\
e10_loose & L1_EM5 & L2_e10_loose & EF_e10_loose \\
e15_loose & L1_EM10 & L2_e15_loose & EF_e15_loose \\
\hline mu4 & L1_MU0 & L2_mu4 & EF_mu4 \\
2mu4 & L1_2MU0 & L2_2mu4 & EF_2mu4 \\
mu6 & L1_MU6 & L2_mu6 & EF_mu6 \\
mu10 & L1_MU10 & L2_mu10 & EF_mu10 \\
\hline \hline
\end{tabular}

Table 4.11: Selected L1, L2, and EF trigger items from the trigger menu that will be used in this analysis. The horizontal row, starting with the name of the trigger chain, represents the input of one trigger hypothesis to the next (that is why for example some L1 triggers are duplicated as they serve as input to several L2 hypotheses).

acceptance threshold for a trigger is low (for example a low $p_{\mathrm{T}}$ cut), then the rate for that particular trigger chain can be too high, flooding the trigger decision and filling the available bandwidth. If a rate becomes too large for a particular trigger item it can be prescaled, or the selection might be tightened, or it might be removed from the menu. A prescaled trigger is one that reduces the number of events that would pass that trigger by some factor to help reduce the overall trigger rate. For example, if a trigger has a prescale of five, then only one in five events that would have passed the trigger are passed to the next analysis stage.

\subsubsection{Electron Triggers}

\section{Level 1:}

At L1 no inner detector information is available, therefore it is not possible to distinguish electrons from photons at this level. The calorimeter creates trigger towers $(0.1 \times 0.1$ in $\Delta \eta \times \Delta \phi$ space $)$ in both the electromagnetic and hadronic calorimeters for $|\eta|<2.5$. Clusters in the electromagnetic and hadronic calorimeter are formed by 
combining $2 \times 2$ of these trigger towers. Properties of these L1 calorimeter clusters are used to quickly classify the amount and type of energy deposition in this region of the detector.

If the minimum $E_{\mathrm{T}}$ is satisfied for any of the triggers the event is said to have passed L1. The pass is recorded for each trigger in which the minimum $E_{\mathrm{T}}$ criteria is satisfied. Associated to each electromagnetic cluster is a region of interest, defined by the $\eta$ and $\phi$ of the $2 \times 2$ trigger tower. The entire calorimeter is scanned for regions of interesting energy deposition in steps of one trigger tower along both $\eta$ and $\phi$.

\section{Level 2:}

The L2 trigger has access to information from the inner tracking detectors. The L2 triggers are given events that pass the $\mathrm{L} 1$ triggers described above. It uses the regions of interest defined by L1, but now has access to the full detector granularity and precision. It runs two algorithms to decide whether to pass the event on to the next trigger level. The first algorithm uses characteristics of the energy deposited in the electromagnetic calorimeter. Using the region of interest from L1 it scans all channels in the region to find the highest $\mathrm{E}_{\mathrm{T}}$ channel and uses this as the seed channel from which an electromagnetic cluster of size $0.075 \times 0.175(\Delta \eta \times \Delta \phi)$ is formed. The following are calorimeter level variables used to make the L2 level decision:

- EM $\mathbf{E}_{\mathrm{T}}=$ transverse energy deposited in the electromagnetic calorimeter (full depth) of size $0.075 \times 0.175(\Delta \eta \times \Delta \phi)$ around the seed channel.

- Had $\mathbf{E}_{\mathrm{T}}=$ transverse energy deposited in the first hadronic calorimeter layer within $0.2 \times 0.2(\Delta \eta \times \Delta \phi)$

- $\mathbf{R}_{\text {core }}=\mathrm{E} 37 / \mathrm{E} 77$, where E37 is the energy deposited in the $3 \times 7$ channels and E77 is the energy deposited in $7 \times 7$ channels in the second electromagnetic 
layer.

The second L2 algorithm uses calorimeter and tracking quantities to match tracks to calorimeter energy depositions to tracks. The quantities in this hypothesis testing are the following:

- $\mathbf{p}_{\mathrm{T}}=$ transverse momentum from the tracking information.

- $\mathrm{E}_{\mathrm{T}} / \mathbf{p}_{\mathrm{T}}=$ the ratio of the $\mathrm{E}_{\mathrm{T}}$ from the calorimeter cluster to $\mathrm{p}_{\mathrm{T}}$ measured by the inner trackers.

- $\Delta \phi=$ difference in $\phi$ between the cluster and extrapolated track to the calorimeter surface.

- $\Delta \eta=$ difference in $\eta$ between the cluster and extrapolated track to the calorimeter surface.

\section{Event Filter:}

Events that pass a L2 trigger are passed to the EF. The EF uses much of the same algorithms as the final offline reconstruction. With respect to L2, it applies improved corrections for detector geometry and energy (such as the crack regions).

\subsubsection{Muon Triggers}

The muon triggers cover $|\eta|<2.4$, and are provided by the RPCs in the barrel region $(|\eta|<1.05)$ and TGCs in the endcap region $(1.05<|\eta|<2.4)$. These detectors have full $\phi$ coverage, except in the regions of $\phi \sim-1,-2$ where support structures of the ATLAS detector lie. There is also a gap in coverage at $\eta=0$ to allow for essential detector services. The magnetic fields, providing curvature to the muon trajectories for momentum measurements, can be split into three regions: (i) $|\eta|<1.4$ with 


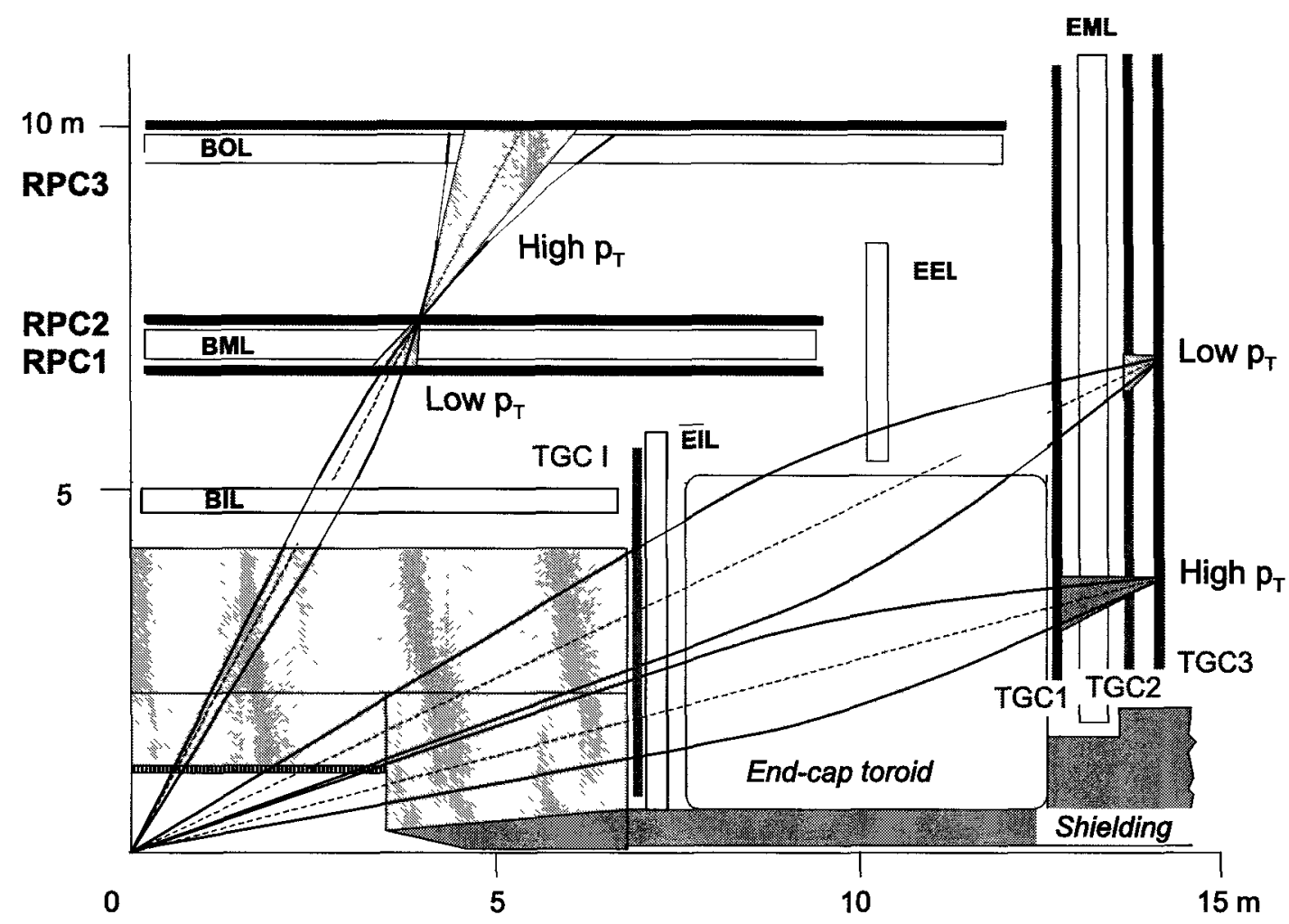

Figure 4.16: Diagram of the muon RPC and TGC trigger layout. The three RPC layers are labeled as RPC1, RPC2, and RPC3. The four TGS layers are labeled as TGCI (innermost), TGC1, TGC2, and TGC3.

the barrel toroids, (ii) $1.4<|\eta|<1.6$ the transition region with an inhomogeneous magnetic field, and (iii) $1.6<|\eta|<2.7$ where the magnetic field is produced by the endcap toroids. Variations in the muon trigger hardware and layout, and changes in the magnetic field can impact the trigger efficiency in each of the different $\eta$ regions, as will be shown in Section 4.5.6. This is different from the case of electrons, where the same detector configuration is provided for the entire $\eta$ range in this analysis.

\section{Level 1:}

The L1 muon trigger decision is hardware based, requiring time and spatial coincidence between the muon trigger detector stations. The layout demonstrating the $\eta$ coverage of the RPC and TGC stations is shown in Figure 4.16. 
In the barrel, there are three stations of RPCs, with increasing radial distance from the interaction point. The barrel toroid lies between RPC2 and RPC3. RPC2 is called the pivot plane, and is the starting point for trigger identification in the barrel. For a hit in the pivot plane a straight line is projected from the hit to the interaction point (denoted as the dashed line in Figure 4.16). The amount of curvature a muon of given momentum would undergo in the known magnetic field is used to define a coincidence window (allowing for both muon polarities) in RPC1 and RPC3 (indicated as shaded region around the dashed projected pivot line in Figure 4.16). If there is a hit in RPC1 within this coincidence window in space and time, a low $p_{\mathrm{T}}$ muon candidate is made. Only then is a search made within RPC3 to locate possible hits within that coincidence window, used to identify high $p_{\mathrm{T}}$ muons. It is important to note that the efficiency of high $p_{\mathrm{T}}$ muon triggers is directly dependent on the ability to first trigger on low $p_{\mathrm{T}}$ muons, as the algorithm first finds hits within RPC1 before searching in RPC3 for the higher momentum tracks.

In the endcap, the TGCs, are located along four planes labeled as TGCI ('inner', with two planes), TGC1 (three planes), TGC2 (two planes), and TGC3 (two planes) in Figure 4.16. Like the RPCs they have a similar method for fast muon triggering using coincidence window measurements. In the TGC, the pivot plane is TGC3, the farthest from the interaction point. Low $p_{\mathrm{T}}$ muons are searched for first in TGC2, and if they pass then a search is made in TGC1 to find high $p_{\mathrm{T}}$ muons. TGCI will be used in the future for high luminosity data to reject backgrounds.

The coincidence windows are defined for six different $p_{\mathrm{T}}$ thresholds. Triggered muons are identified by which $p_{\mathrm{T}}$ threshold they satisfy, and are passed with the threshold and geometrical region of interest to the HLT for further processing. The name of the L1 muon trigger contains the $p_{\mathrm{T}}$ threshold, so L1_MU6 requires a muon with a minimum $p_{\mathrm{T}}$ of $6 \mathrm{GeV}$ to pass. The trigger L1_MU0 does not have any $p_{\mathrm{T}}$ 
requirement, only time coincidence between the muon trigger detectors.

\section{HLT:}

The muon HLT is software based, and starts at L2 seeded by the regions of interest from L1. L2 uses the full muon spectrometer information, adding the MDT and CSC to identify muon tracks and estimate the $p_{\mathrm{T}}$. It then combines the muon spectrometer tracks to tracks from the inner detector. Isolation criteria can be included by using information from the calorimeter. The EF uses similar algorithms as L2, however more time is available to make better track reconstruction and momentum calculations. As with the L1 muon trigger, the naming of the HLT indicates the $p_{\mathrm{T}}$ threshold, so L2_mu10 requires a muon with transverse momentum of $10 \mathrm{GeV}$ to pass.

\subsubsection{Trigger Efficiencies}

Throughout this section the trigger efficiency is defined as:

$$
\epsilon_{T}=\frac{\text { number of objects or events passing selection criteria AND trigger of interest }}{\text { number of objects or events passing selection criteria }}
$$

The two types of trigger efficiencies of interest are:

1. The efficiency for an object (electron, muon, jet, etc.) to be triggered as a function of various kinematic criteria of the object. This is achieved in the MC by looping through all the final reconstructed objects of interest and seeing if the trigger was fired for the event. If the event was triggered it is necessary to loop through all of the trigger regions of interest and match (by a $\Delta R$ criteria) to the reconstructed object. This basic $\Delta R$ matching should be looser for L1 and tighter for the $\mathrm{EF}$, as the granularity and precision increases with each trigger level. It is important to understand any biases the trigger might have 
for particular objects and associated kinematics.

2. The efficiency of an event to pass the trigger criteria. This is ultimately an input into the cross-section measurement. In most cases, the efficiency will be less than one, and so the observed final number of events is reduced.

For the remainder of this section, a reconstructed electron or muon is required to pass the criteria previously listed in Tables 4.5 and 4.7 , except no truth matching is required, and for certain situations the $p_{\mathrm{T}}$ cut is modified.

An example of the $\Delta R$ between the trigger and reconstructed lepton is shown in Figure 4.17 for the e5medium trigger chain with reconstructed electrons, and the mu6 trigger chain with muons, for the three trigger levels. The events contributing to these plots are the true dilepton events. The reconstructed electrons and muons do not have any minimum $p_{\mathrm{T}}$ requirement (the plots reveal the same nature when a $p_{\mathrm{T}}$ cut is introduced). Similar trends hold for the trigger levels of the other triggers of interest (listed in Table 4.11). The $\Delta R$ between a reconstructed object and a L1 item tends to be larger than the other levels, as L1 suffers from reduced detector granularity. Moving up the chain towards the EF more information and better calibration are applied to the trigger reconstruction, bringing each step closer to the final reconstructed object. In the subsequent analysis the trigger requirement is that the trigger object must pass the event and the trigger must match a reconstructed object within $\Delta R<0.1$ for $\mathrm{EF}, \Delta R<0.15$ for $\mathrm{L} 2$, and $\Delta R<0.2$ for L1, for both electrons and muons.

\section{Trigger Turn-On Curves and Object Efficiencies}

The common cut applied at each trigger level is a cut on the total transverse energy for electrons and transverse momentum for muons (electrons are treated as massless objects, so the transverse momentum and transverse energy are equivalent). At each 


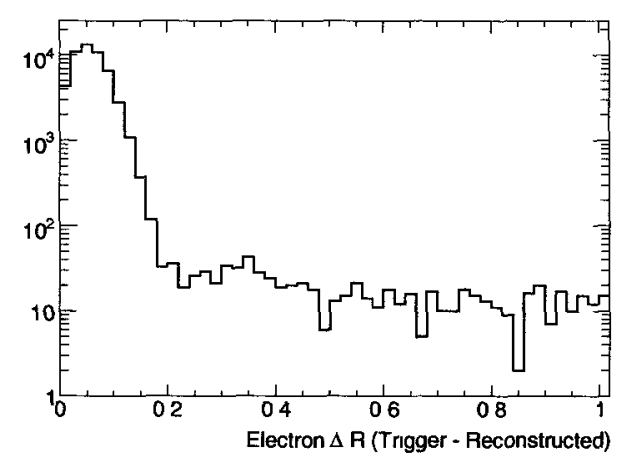

(a) L1

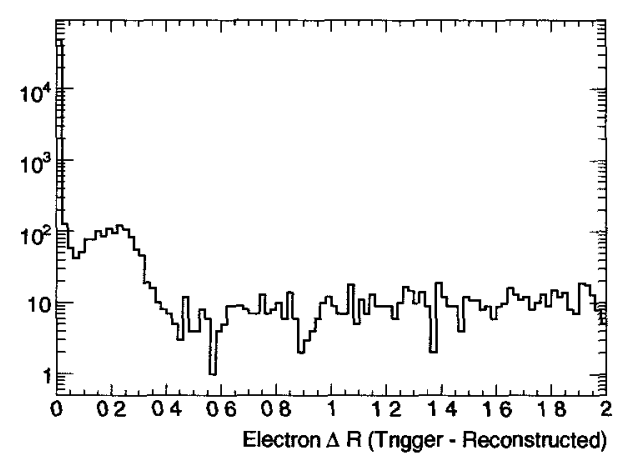

(c) L2

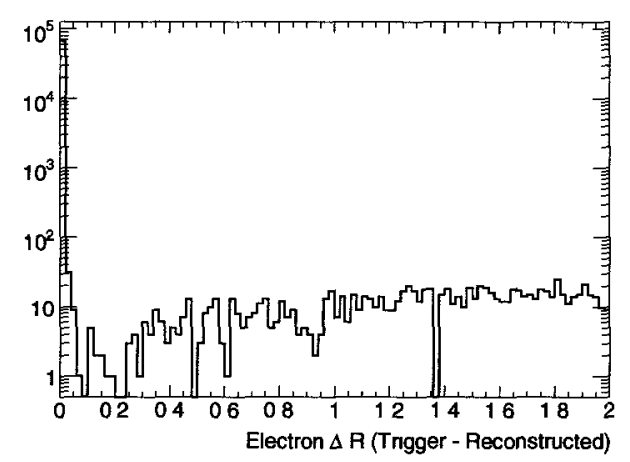

(e) EF

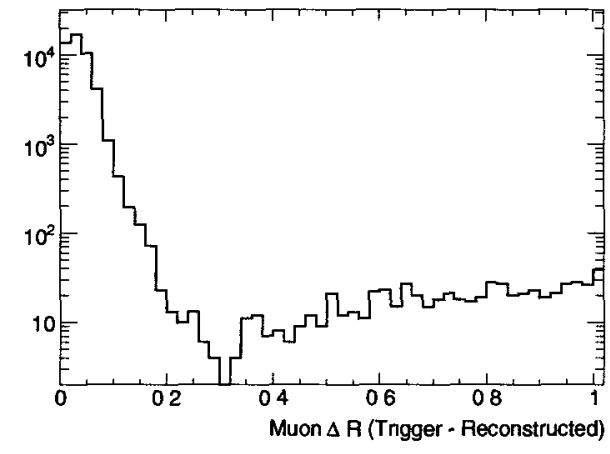

(b) L1

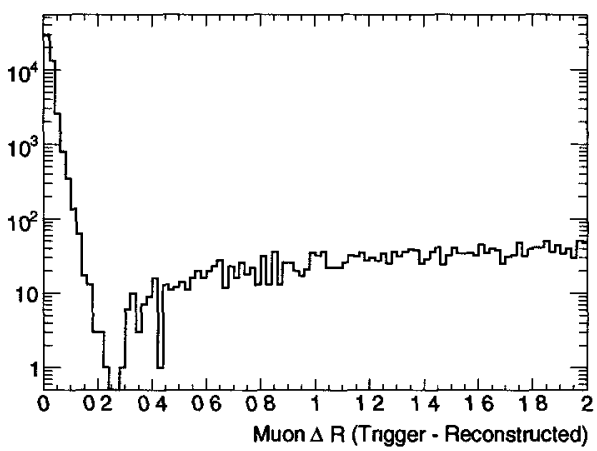

(d) $\mathrm{L} 2$

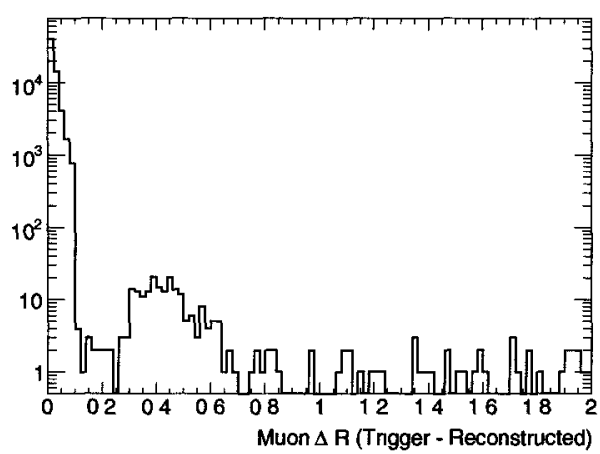

(f) $\mathrm{EF}$

Figure 4.17: Shown on the left is the $\Delta R$ between reconstructed electrons and the closest trigger region for each trigger level in the e5_medium trigger chain for each trigger level. Shown in the right column is the $\Delta R$ between reconstructed muons and the closest trigger region for each trigger level in the mu6 trigger chain. No $p_{\mathrm{T}}$ cuts have been applied to the reconstructed objects. 
level the trigger must make the decision to accept or reject the event in $2.5 \mu$ s at L1, $40 \mathrm{~ms}$ at L2 and 4 seconds at the EF. The extended algorithms applied offline for calibrations and corrections sometimes cannot be applied, which degrades the resolution of the trigger reconstruction leading to differences between the trigger and offline values of the reconstruction $E_{\mathrm{T}}$. It is therefore preferred to apply an offline $E_{T}$ cut that is slightly larger than that of the chosen trigger. For example, if a trigger requires the reconstructed object to have $E_{T}>15 \mathrm{GeV}$, the user should apply an offline selection criteria of $E_{T}>20 \mathrm{GeV}$ to avoid the window of uncertainty in the trigger decision. The choice of offline $E_{T}$ cut is based on examining trigger efficiency turn-on curves, which plot the efficiency as a function of reconstructed $E_{T}$ for each reconstructed object.

The final trigger selection before the reconstruction is applied at the EF level, so efficiencies will be calculated for the EF level with respect to the offline reconstruction. An event that passes the EF must have also passed the earlier L1 and L2 trigger items of their trigger chain.

\subsubsection{Object Trigger Efficiencies: Single Lepton Triggers}

This section examines the object trigger efficiencies that require only a single lepton to pass the trigger criteria.

Trigger object efficiencies are the efficiency of the trigger with respect to the offline reconstruction. These can be channel dependent, as the efficiency for a trigger with isolation requirements may be less for a channel with more activity in the final state. The different dilepton $t \bar{t}$ channels each have similar kinematics, only varying by the production of an electron or muon or tau lepton. It is possible that electrons and muons produced from tau leptons may have slightly different kinematics, but 


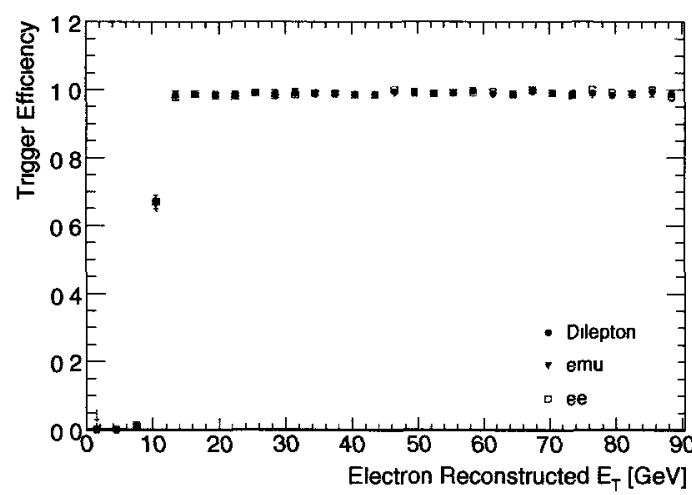

(a) EF_e10_medium

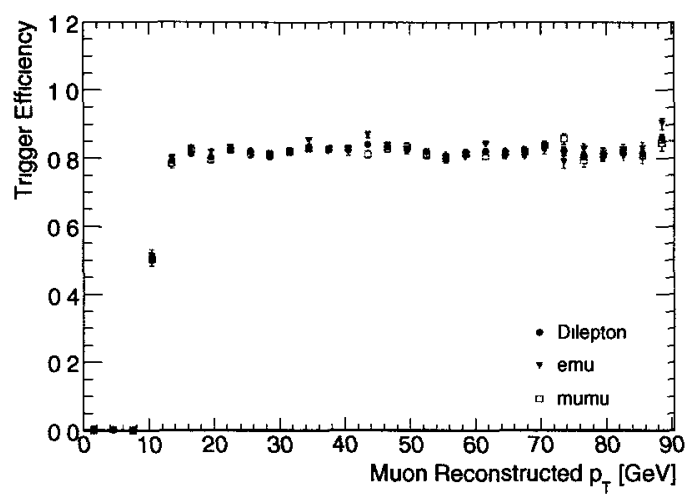

(b) EF_mu10

Figure 4.18: Trigger efficiency turn-on curves for the (a) EF_e10_medium and (b) EF_mu10 triggers, for selected true $t \bar{t}$ dilepton channels (as shown on the legends). The object efficiencies are the same (within statistics) for all of the different $t \bar{t}$ dilepton final states. The dilepton final states have been selected using truth information.

this difference will be ignored. Sample plots of the trigger efficiency versus offline reconstructed electron $E_{\mathrm{T}}$ and muon $p_{\mathrm{T}}$ for different dilepton channels are shown in Figure 4.18. Figure 4.18(a) shows the electron trigger efficiency for the $e e, e \mu$, and all dilepton channels for the EF_e10_medium trigger, and in (b) the muon trigger efficiencies for the $\mu \mu, e \mu$, and all dilepton channels with the EF_mu10 trigger. The dilepton channels have been selected from the truth information. These plots indicate that for the $t \bar{t}$ dilepton channel the electron and muon object trigger efficiencies are the same for the different final states. As a reminder, electrons are treated as massless objects, hence $E_{\mathrm{T}}=p_{\mathrm{T}}$.

The electron triggers have a high efficiency of triggering on reconstructed electrons, near $99 \%$. The muon triggers are less efficient with an efficiency of around $83 \%$. Trigger inefficiencies in the muon trigger are the result of transitions in the magnetic field and regions of reduced trigger detector coverage.

The trigger efficiencies shown in Figure 4.18 are examples of trigger turn-on curves, showing the trigger efficiency as a function of reconstructed $p_{\mathrm{T}}$. In an analysis the 


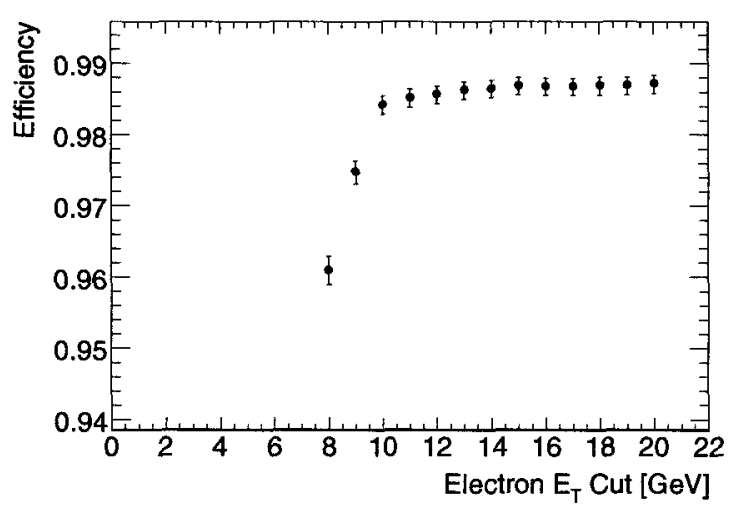

(a) EF_e10_medium

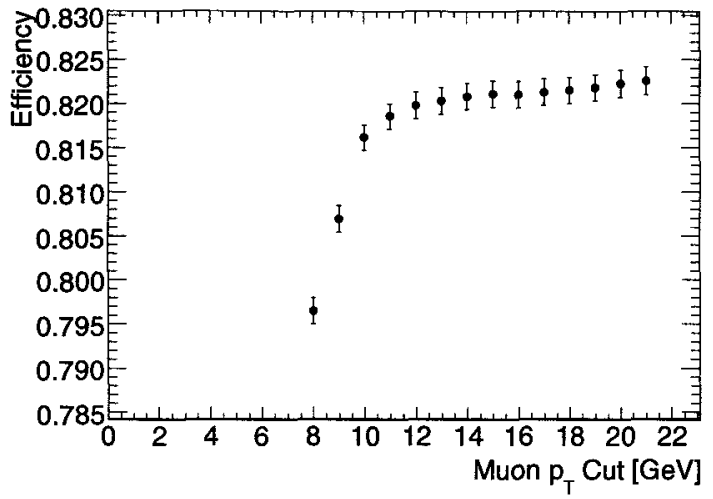

(b) EF_mu10

Figure 4.19: (a) Trigger efficiency for electrons with the EF_e10_medium trigger as a function of $E_{\mathrm{T}}$ cut applied to the reconstructed electron, and (b) the muon trigger efficiency using EF_mu10 trigger as a function of $p_{\mathrm{T}}$ cut applied to the reconstructed muon.

user will apply a $p_{\mathrm{T}}$ cut to the reconstructed objects, this value should be in the trigger turn-on curve plateau to avoid biases near the turn-on region. Figure 4.19 shows the overall trigger efficiency for electrons and muons for the EF_e10_medium and $\mathrm{EF} \_$mu10 triggers as a function of the $E_{\mathrm{T}}$ or $p_{\mathrm{T}}$ cut applied to the reconstructed particle. The EF_e10_medium reaches a plateau when a cut of approximately $12 \mathrm{GeV}$ in $E_{\mathrm{T}}$ is applied to the offline reconstructed electron. For the EF mu10 trigger the plateau is closer to about $14 \mathrm{GeV}$. The object trigger efficiency is shown in Table 4.12. In this table the first column indicates the trigger name, the second column states the $p_{\mathrm{T}}$ cut, and the third column shows the object trigger efficiency at that $p_{\mathrm{T}}$ cut (which falls in the plateau region).

The trigger efficiencies as a function of other lepton kinematic variables are shown in Figure 4.20 for electrons and 4.21 for muons (for one example trigger). In the figures the points indicate the trigger efficiency and the solid histograms show the distributions contributing to the denominator of the efficiency calculation, with arbitrary normalization (to demonstrate the shape).

The electron plots show the trigger efficiency with respect to the electron $E_{\mathrm{T}}, \eta$, 


\begin{tabular}{|l|c|c|}
\hline Trigger Name & Cut Value [GeV] & Trigger Efficiency [\%] \\
\hline \hline EF_e5_medium & 10 & 98.9 \\
EF_e10_medium & 15 & 98.7 \\
EF_e10_loose & 15 & 99.1 \\
EF_e15_loose & 20 & 99.2 \\
EF_mu4 & 10 & 84.3 \\
EF_mu6 & 10 & 82.6 \\
EF_mu10 & 15 & 82.1 \\
\hline \hline
\end{tabular}

Table 4.12: Object trigger efficiency in the plateau region of the trigger turn-on curves for the $t \bar{t}$ dilepton channels, for various cuts to the reconstructed object $E_{\mathrm{T}}$ (electrons) and $p_{\mathrm{T}}$ (muons). For the electron triggers the object is offline reconstructed electrons, and for muons it is offline reconstructed muons. The statistical errors are insignificant and are not shown (of order $10^{-3} \%$ ).

$\phi$, and electron isolation variable defined in Equation 4.5. All four plots show that the electron trigger efficiency is high and flat across the kinematical variables used to select electrons. The dip in the $\eta$ histogram at $|\eta| \sim 1.5$ reflects the exclusion of this region in the selection of reconstructed electrons (recall this is the transition region between the barrel and endcap electromagnetic calorimeters).

The muon plots in Figure 4.21 indicate the trigger efficiencies vary with $\eta$ and $\phi$. In $\eta$, the histogram has a dip at $\eta \sim 0$ as there are no muon spectrometer detectors in this gap region. This affects not only the ability to reconstruct muons, but also in the trigger efficiency with respect to the offline reconstruction. The other features in $\eta$ reflect transitions of the magnetic field. The lack of muon triggers near the ATLAS support structures at $\phi$ around -1 and -2 lead to the inefficiency in these regions. These plots are with respect to the offline reconstruction, which can make use of the inner detector and calorimeter to assist in identifying muon candidates. The L1 trigger which is used to make the initial trigger decision serves as input to all subsequent trigger levels and only has access to the muon spectrometer information; gaps or transitions in muon RPC or TGC coverage will be reflected in these plots. 


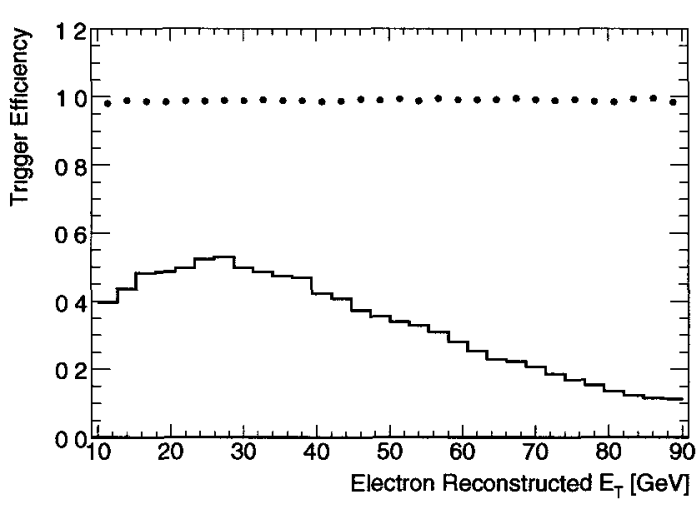

(a)

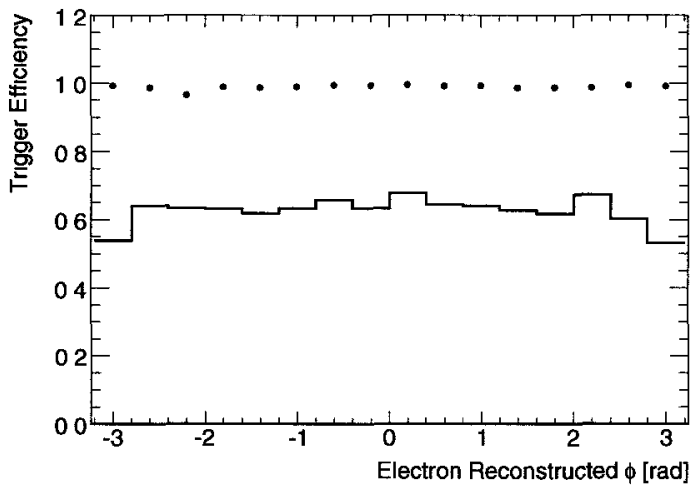

(c)

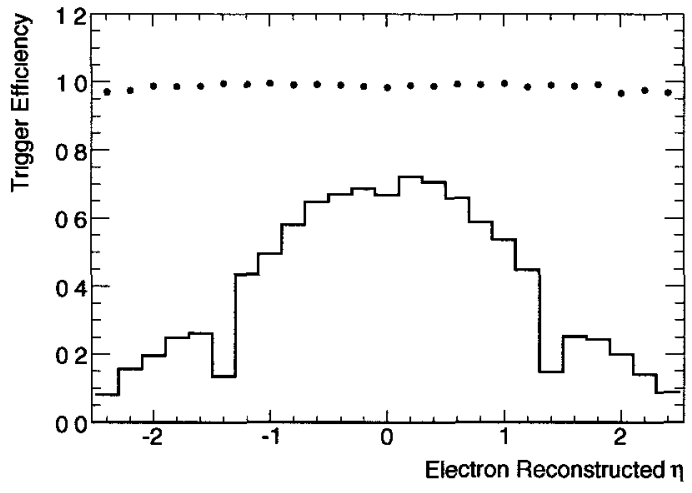

(b)

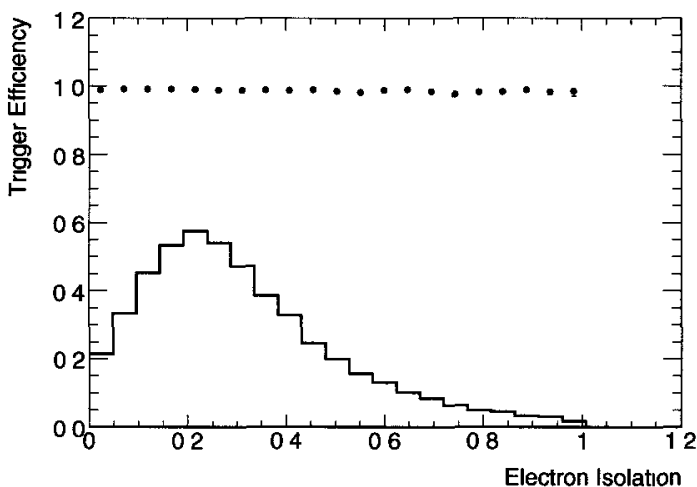

(d)

Figure 4.20: The solid points show the electron trigger efficiency (shown for EF_e5_medium) as a function of reconstructed electron $E_{\mathrm{T}}, \eta, \phi$, and the electron isolation variable defined in Equation 4.5. The qualities of these plots are similar for the other electron triggers (with possible variations on the initial $E_{\mathrm{T}}$ value to select the phase space in the plateau region of the trigger turn-on curve). The solid histograms represent the distributions of the electrons before the trigger requirement, to some arbitrary normalization. 


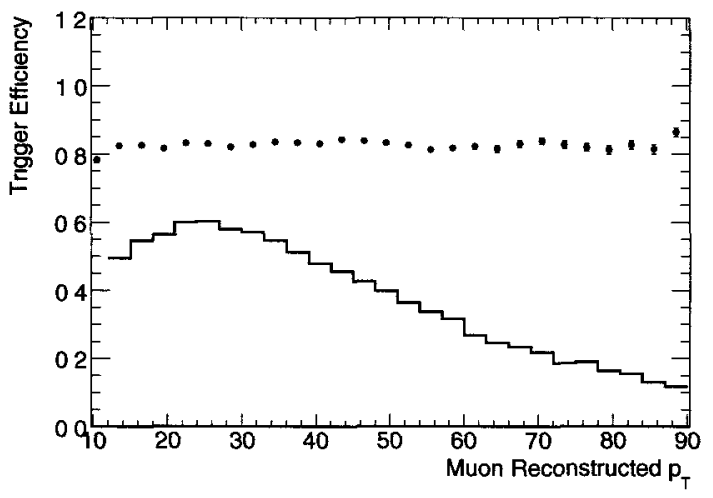

(a)

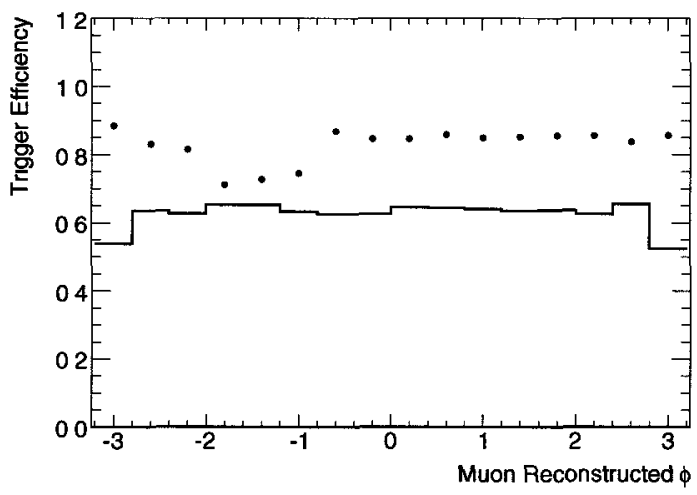

(c)

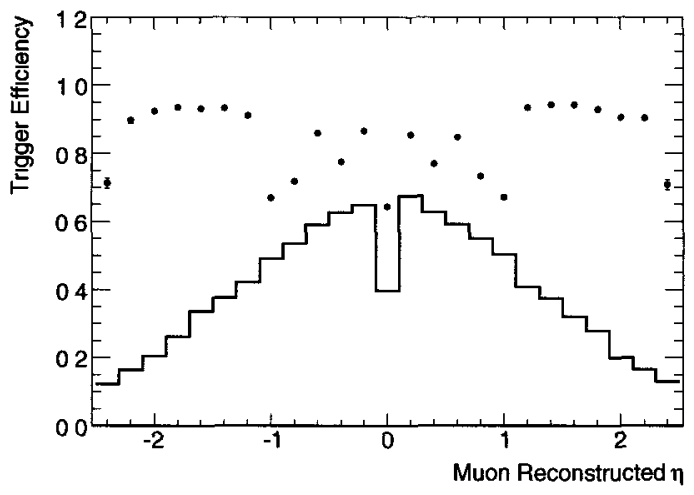

(b)

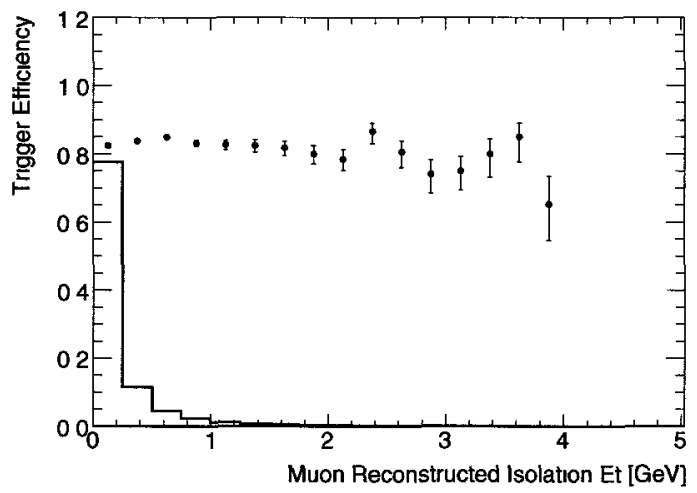

(d)

Figure 4.21: The solid points show the muon trigger efficiency (shown for EF_mu6) as a function of reconstructed muon $p_{\mathrm{T}}, \eta, \phi$, and calorimeter isolation. For these plots the EF_mu6. Not shown is the muon trigger efficiency with respect to the muon $p_{\mathrm{T}}$ isolation variable, which is also flat. The solid histograms represent the distributions of the muons before the trigger requirement, to some arbitrary normalization. 


\subsubsection{Object Trigger Efficiencies: Dilepton Triggers}

The $t \bar{t}$ dilepton signal contains two leptons in the final state, so it is possible to make use of dilepton triggers. For an event to be included in the dilepton trigger efficiency calculation it must contain two reconstructed leptons of that dilepton trigger type. For every event that satisfies this minimum criteria, all of the reconstructed objects are used in the trigger efficiency calculation, Equation 4.5.5. If the two leptons in the event are produced close together, the trigger will not be able to distinguish the two objects, and hence only the single trigger may fire. The dilepton triggers considered here are EF_2e5_medium and EF_2mu4. For the final state containing an electron and muon the trigger that will be proposed in future sections is an AND trigger requiring both EF_e5_medium and EF_mu4 triggers to fire in the event. Since these two trigger systems are independent, the object (electron and muon) trigger efficiencies for the combination trigger is the product of the individual single triggers. This combination trigger does not currently exist in the ATLAS trigger menu, but motivation will be provided in Section 4.6 to consider this as a useful trigger.

The di-electron trigger (EF_2e5_medium) efficiency as a function of electron kinematic variables is shown in Figure 4.22. An $E_{\mathrm{T}}$ cut of $10 \mathrm{GeV}$ is placed on the reconstructed electrons, to ensure the $E_{\mathrm{T}}$ efficiency is in the plateau region of the trigger turn-on curve. The overall electron trigger efficiency is $97.7 \%$ for the dilepton events, which is lower than the probability for the single lepton trigger fire.

The muon trigger efficiencies for the EF_2mu4 trigger are plotted in Figure 4.23. These efficiencies show the same features as the single muon triggers. The object trigger efficiency for EF_2mu4 is $73.9 \%$. 


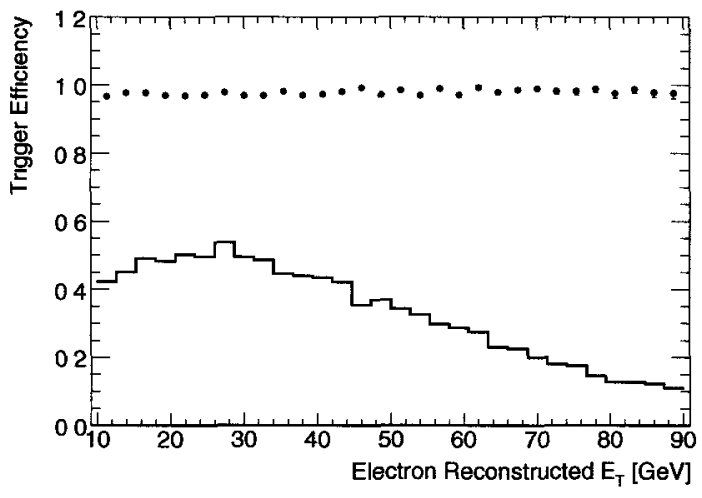

(a)

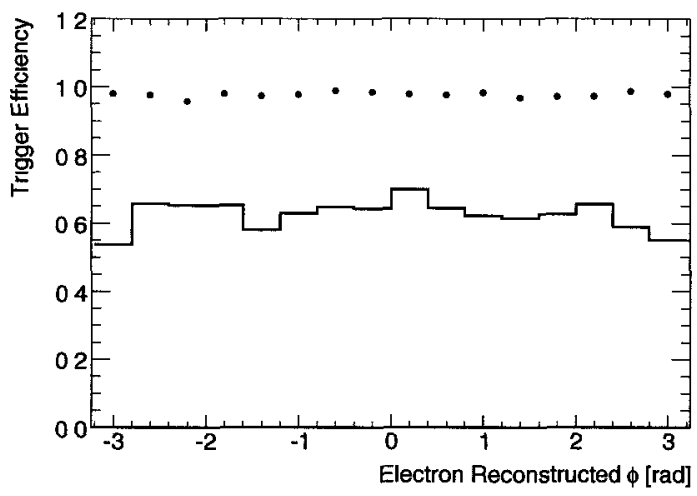

(c)

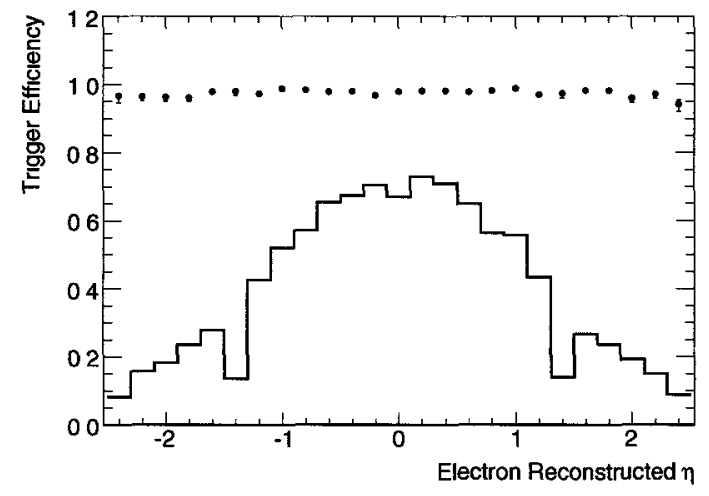

(b)

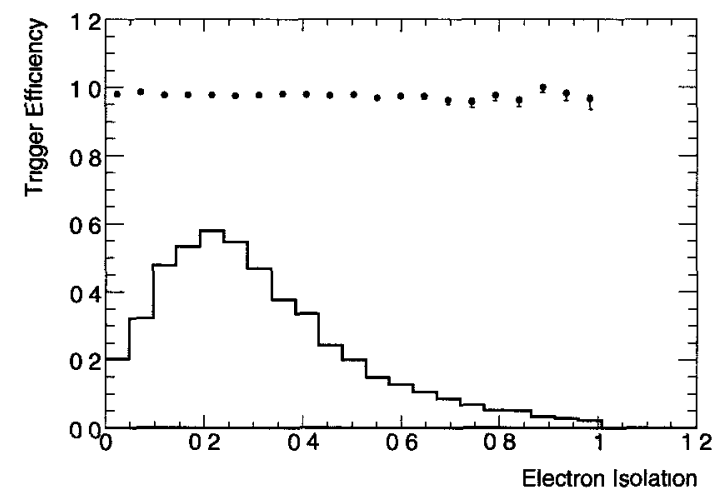

(d)

Figure 4.22: The solid points show the electron trigger efficiency for EF_2e5_medium, as a function of reconstructed electron $E_{\mathrm{T}}, \eta, \phi$, and the electron isolation variable defined in Equation 4.5. The solid histograms represent the distributions of the electrons before the trigger requirement, to some arbitrary normalization. 


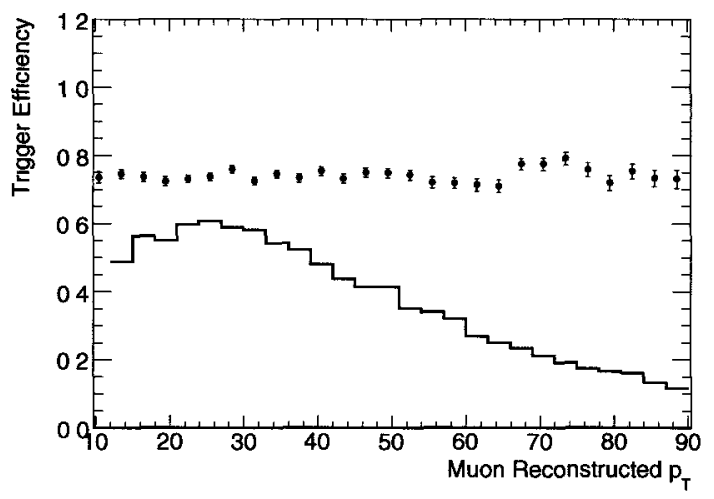

(a)

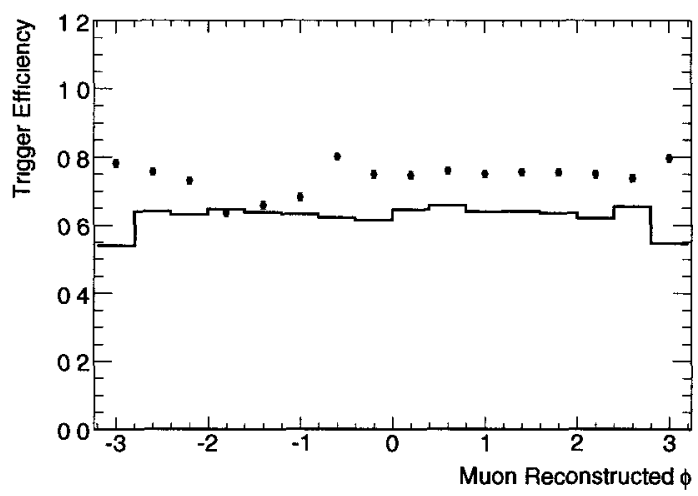

(c)

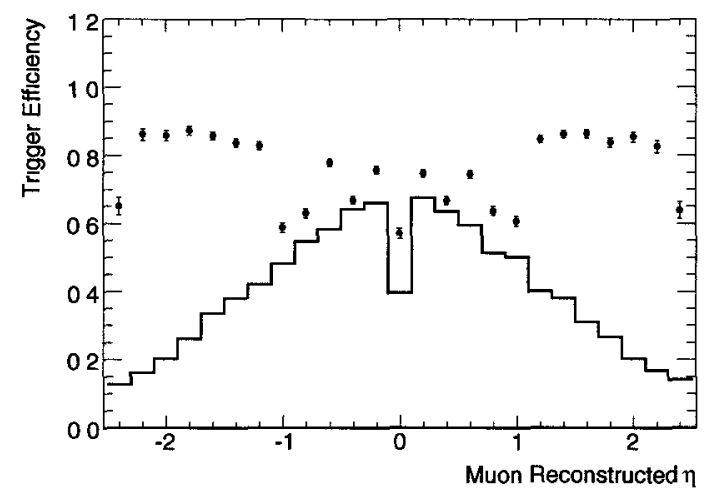

(b)

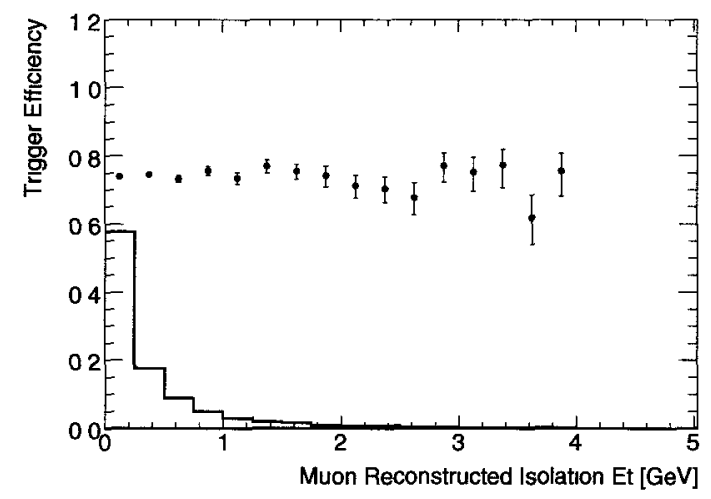

(d)

Figure 4.23: The solid points show efficiency for the EF_2mu4trigger as a function of reconstructed muon kinematics: a) $p_{\mathrm{T}}$ b) $\eta$ c) $\phi$, and d) muon isolation in $E_{\mathrm{T}}$. Not shown is the muon trigger efficiency with respect to the muon $p_{\mathrm{T}}$ isolation variable, however that distribution is flat. The solid histograms represent the distributions of the muons before the trigger requirement, to some arbitrary normalization. 


\subsubsection{Object Trigger Efficiencies Summary}

The electron triggers, requiring either a single or double electron signature, all behave with high trigger efficiency and are flat across the electron kinematic quantities used for the selection of electrons. Therefore, there are no biases in the electron triggers based on the selection of the electron item for the analysis. Later in the analysis, when the event trigger efficiencies are computed this can be applied as a single number to the cross-section calculation, without dependence on the specific electrons in the event.

The situation is different for muons, in which the trigger efficiencies do depend on the $\eta$ and $\phi$ of the muon(s) in the event. The $\eta$ distribution of reconstructed muons is peaked towards the center, however the trigger efficiency in this region is reduced and exhibits a significant structure with respect to the endcaps. There is also the asymmetry in $\phi$ that needs to be taken into account.

A further discussion of the application of trigger efficiencies to individual events is made in Section 4.7.2.

\subsection{Event Selection and Expected Number of Sig- nal and Background Events}

The $t \bar{t}$ channel has a complex final state with leptons, jets, and missing transverse energy. Kinematical quantities of these objects can be used to select the $t \bar{t}$ channel, and hopefully this can be done while minimizing contributions from background processes. The largest source of backgrounds for the dilepton analysis are from the $W \rightarrow l \nu+$ jets and $Z \rightarrow l l+$ jets processes. These both have cross-sections larger than the signal and have final states that resemble the $t \bar{t}$ channel. The signal channel containing two isolated electrons in the final state is called the ee channel. Similar 


\begin{tabular}{|l||c|c|c|}
\hline Variable & $e e$ & $\mu \mu$ & $e \mu$ \\
\hline \hline Number of Leptons & $e^{+} e^{-}$ & $\mu^{+} \mu^{-1}$ & $e \mu(\mathrm{OS})$ \\
Dilepton Invar. Mass Cut & $86 \mathrm{GeV}$ to $96 \mathrm{GeV}$ & $81 \mathrm{GeV}$ to $101 \mathrm{GeV}$ & - \\
Missing $E_{\mathrm{T}}$ cut & $>35 \mathrm{GeV}$ & $>35 \mathrm{GeV}$ & $>20 \mathrm{GeV}$ \\
Min. of Num Jets & two & two & two \\
\hline \hline
\end{tabular}

Table 4.13: Event selection criteria for the $t \bar{t}$ dilepton $e e, \mu \mu$, and $e \mu$ channels. A note on the abbreviations: OS stands for opposite sign, Min stands for minimum, and Invar stands for invariant. The leptons, either electron or muon, are required to satisfy the criteria in Table 4.5 and 4.7 (except the truth matching criteria).

definitions exist for the $\mu \mu$ and $e \mu$ channels.

The final selection is channel dependent $(e e, \mu \mu, e \mu)$ as the contribution from backgrounds is different for each channel. The $e e$ and $\mu \mu$ channels closely resemble the final state of the $Z \rightarrow l l+$ jets background (especially the $Z \rightarrow l l+2$ jet final state), so cuts are placed on the invariant mass of the dileptons around the $Z$ mass.

The event selection criteria for each of the three signal channels of interest are listed in Table 4.13. Each channel requires exactly two opposite sign leptons in the final state (with family reflective of the particular channel). The invariant mass cut and missing transverse energy cut have been optimized by the ATLAS top quark cross-section group to provide the best ratio of the number signal $(S)$ to background $(B)$ events and significance of the signal $(S / \sqrt{S+B})$. It was found the $Z \rightarrow l l+$ jets background was much larger in the $\mu \mu$ channel than the ee channel, in large part due to the higher efficiency of reconstruction muons ( $\sim 63 \%$, Section 4.4 .3$)$ as compared to electrons $(\sim 53 \%$, Table 4.6$)$. Therefore to reduce the contribution from $Z$ events to the $\mu \mu$ channel a larger cut is placed around the invariant mass of the two muons around the $Z$ mass.

In the following study, the number of signal and background events observed are those assuming an integrated luminosity of $200 \mathrm{pb}^{-1}$ (luminosity will be discussed 
in Section 4.7.3). The initial number of events used for the analysis are shown in Table 4.14 for each physics processes. These numbers were then scaled appropriately for $200 \mathrm{pb}^{-1}$. The full MC statistics were used for the $t \bar{t}$ signal sample. For all but the $W \rightarrow l \nu$ and QCD sample, the luminosity scaling factors were less than one. Constraints on computing processing time and storage limited the use of the full available statistics for the background MC data sets. The QCD physics process has an extremely large cross-section $\left(\mathrm{O}\left(10^{11} \mathrm{pb}\right)\right)$, so there is certainly a lack of $\mathrm{MC}$ statistics for this background. Jets in QCD can resemble the signal by faking the signature of electrons and/or muons, and significant missing transverse energy can be present from miscalibrations of the jet energies. The probability for jets to fake electrons and muons must be understood to really quantify the contribution from this background. Even if the fake rate is low, with such a large cross-section the impact could be significant. In this analysis, with the limited statistics available, no QCD events survived any channel event selection. However, future work should further investigate this potential source of background.

Selected event kinematics, such as lepton $p_{\mathrm{T}}$, missing $E_{\mathrm{T}}$, dilepton invariant mass, and jet multiplicity are shown for the signal and backgrounds events, in the ee, $\mu \mu$, and $e \mu$ channels in Figures 4.24, 4.25, and 4.26, respectively. The distributions contain all of the event selection criteria for the dilepton channel. The histograms have been stacked. Note that no trigger requirements have been applied for the data shown in the histograms.

The invariant mass distributions for the $e e$ and $\mu \mu$ channels show a dip at approximately $90 \mathrm{GeV}$, where the cut around the $Z$ boson mass has been applied. For the $e e$ and $\mu \mu$ channels the largest remaining backgrounds are from $Z \rightarrow l l+$ jets. The event selection in the $e \mu$ channel does a good job of reducing all backgrounds.

The choice of trigger can affect the total number of observed events due to trigger 


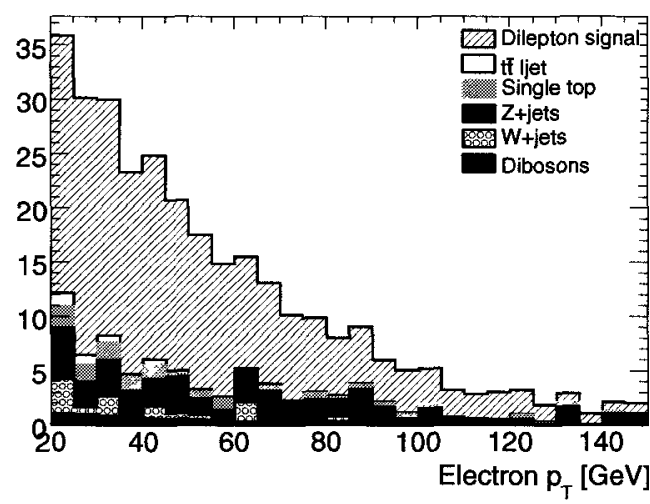

(a)

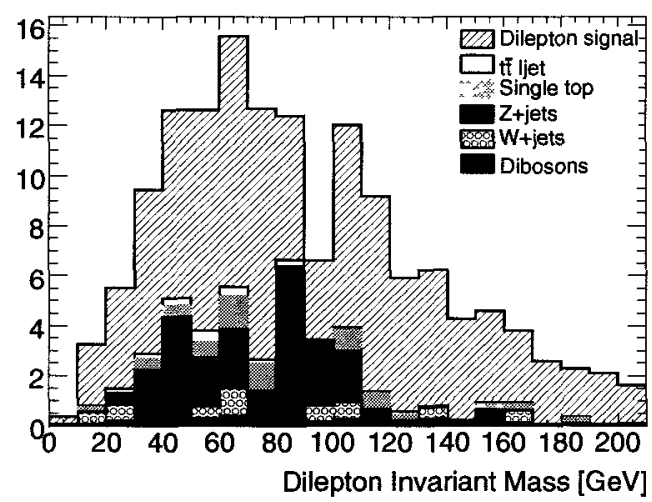

(c)

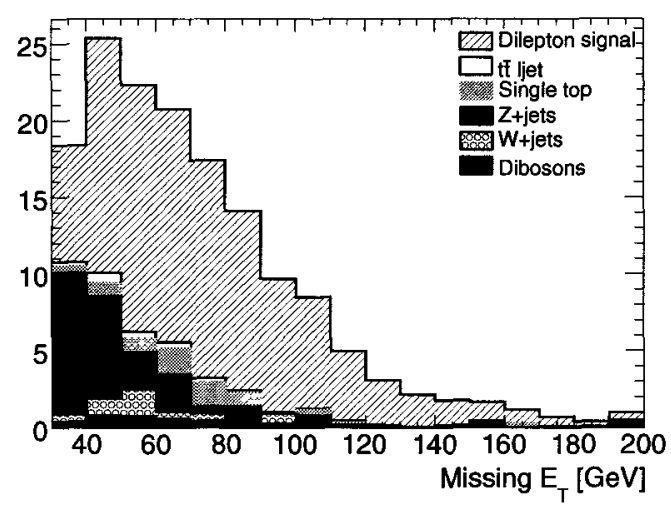

(b)

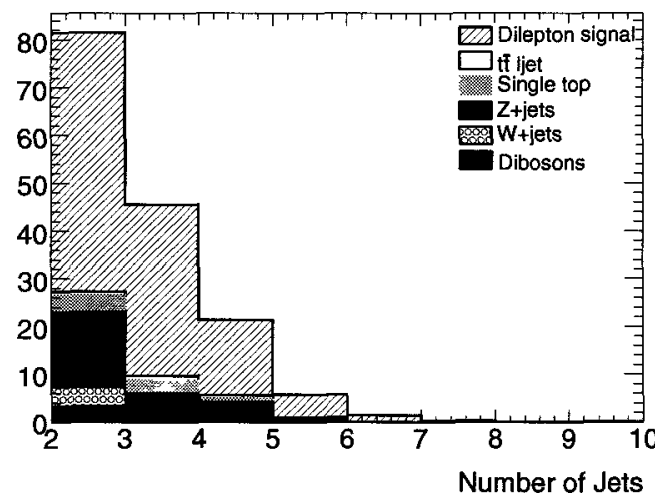

(d)

Figure 4.24: (a) Electron $p_{\mathrm{T}}$, (b) event missing $E_{\mathrm{T}}$, (c) the di-electron invariant mass, and (d) the number of jets per event for the ee channel. 


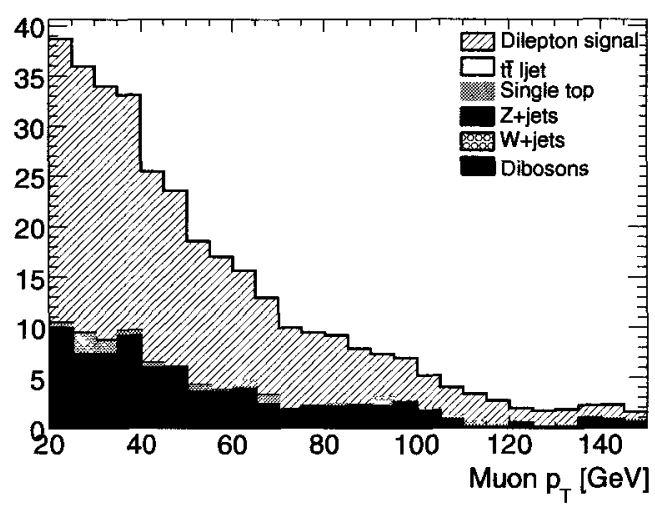

(a)

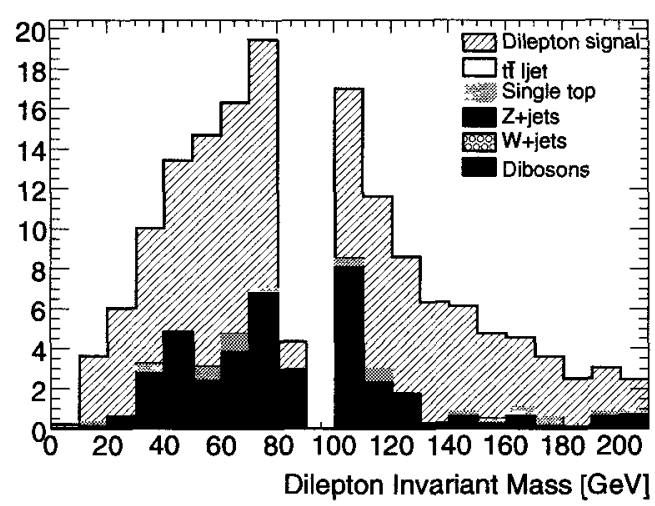

(c)

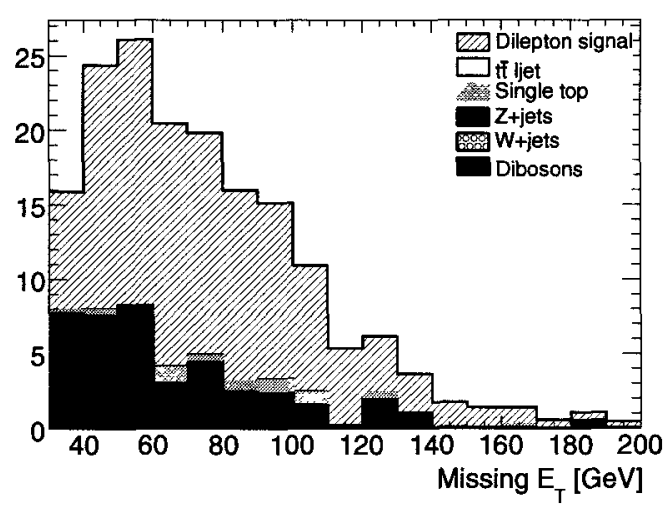

(b)

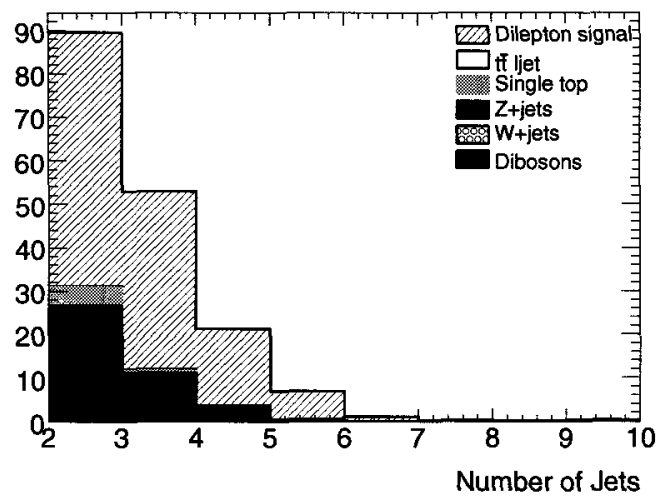

(d)

Figure 4.25: (a) Muon $p_{\mathrm{T}}$, (b) event missing $E_{\mathrm{T}}$, (c) the di-muon invariant mass, and (d) the number of jets per event for the $\mu \mu$ channel.

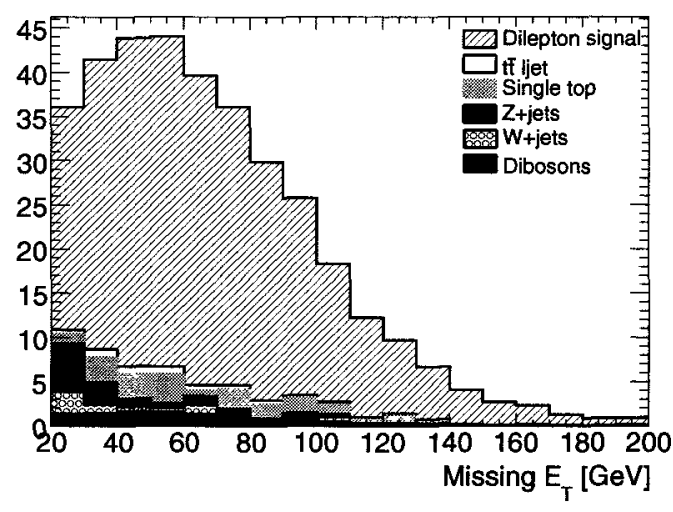

(a)

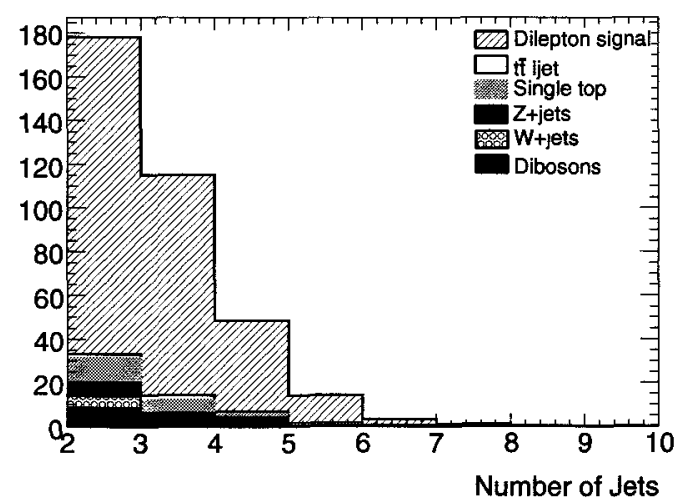

(b)

Figure 4.26: (a) Missing $E_{\mathrm{T}}$ and (b) the number of jets per event for the $e \mu$ channel. 


\begin{tabular}{|r|c|}
\hline & Number of Events \\
\hline \hline$t \bar{t}$ dilepton $+t \bar{t}$ ljet & 773167 \\
Single top & 56469 \\
$t \bar{t}$ fully hadronic & 31006 \\
\hline$W \rightarrow e \nu+$ jets & 481341 \\
$W \rightarrow \mu \nu+$ jets & 480765 \\
$W \rightarrow \tau \nu+$ jets & 479542 \\
\hline$Z \rightarrow e e+$ jets & 261239 \\
$Z \rightarrow \mu \mu+$ jets & 270630 \\
$Z \rightarrow \tau \tau+$ jets & 300835 \\
\hline$W W \rightarrow(l \nu)(l \nu)$ & 99830 \\
$W Z \rightarrow($ incl $)(l l)$ & 34987 \\
$Z Z \rightarrow($ incl $)(11)$ & 21988 \\
\hline QCD & 574341 \\
\hline
\end{tabular}

Table 4.14: The total number of MC events used for the signal and each of the backgrounds. These numbers were scaled to give the expected number of events with $200 \mathrm{pb}^{-1}$ of LHC $\operatorname{data}(\sqrt{s}=7 \mathrm{TeV})$.

inefficiencies, and because the $p_{\mathrm{T}}$ cut applied to the reconstructed objects should be in the plateau region of the trigger turn-on curve. In the discussion of the rest of the thesis an event passing the trigger condition must also have at least one reconstructed lepton match a trigger region of interest of the EF with $\Delta R<0.1$. For single lepton triggers only one match is required, for dilepton triggers the requirement is two matching leptons.

First looking at the ee channel, the triggers considered are the EF_e10_medium and EF_2e5_medium triggers. Using the EF_e10_medium trigger one should apply a minimum $p_{\mathrm{T}}$ cut of $15 \mathrm{GeV}$ to the reconstructed electrons to avoid trigger biases in the turn-on region (Figure 4.19). For the EF_2e5_medium trigger this cut should be $10 \mathrm{GeV}$. Table 4.15 breaks down the signal and background contributions to the $e e$ channel using both of these triggers, with the electron $p_{\mathrm{T}}$ cuts mentioned in the 


\begin{tabular}{|r||c|c|c|c|}
\hline & $\begin{array}{c}\text { EF_e10_medium } \\
\left(p_{\mathrm{T}}>15 \mathrm{GeV}\right)\end{array}$ & $\begin{array}{c}\text { EF_e10_medium } \\
\left(p_{\mathrm{T}}>20 \mathrm{GeV}\right)\end{array}$ & $\begin{array}{c}\text { EF_2e5_medium } \\
\left(p_{\mathrm{T}}>10 \mathrm{GeV}\right)\end{array}$ & $\begin{array}{c}\text { EF_2e5_medium } \\
\left(p_{\mathrm{T}}>20 \mathrm{GeV}\right)\end{array}$ \\
\hline \hline$t \bar{t} \rightarrow$ dilepton & 134.2 & 112.1 & 152.0 & 109.5 \\
\hline$t \bar{t} \rightarrow l$ jet & 5.4 & 2.7 & 12.4 & 2.5 \\
$W \rightarrow l \nu+$ jets & 7.2 & 5.0 & 13.5 & 5.0 \\
$Z \rightarrow l l+$ jets & 31.4 & 24.0 & 38.6 & 22.9 \\
Dibosons & 5.7 & 4.6 & 6.6 & 4.6 \\
Single top & 8.6 & 7.3 & 9.7 & 7.3 \\
\hline signal $(S)$ & $134.2 \pm 1.7$ & $112.1 \pm 1.6$ & $152.0 \pm 1.9$ & $109.5 \pm 1.6$ \\
background $(B)$ & $58.3 \pm 5.0$ & $43.6 \pm 4.4$ & $80.8 \pm 5.8$ & $42.3 \pm 4.3$ \\
$S / B$ & 2.30 & 2.57 & 1.88 & 2.59 \\
$S / \sqrt{S+B}$ & 9.7 & 9.0 & 10.0 & 8.9 \\
\hline \hline
\end{tabular}

Table 4.15: Signal and background event numbers after the ee event selection for $200 \mathrm{pb}^{-1}$ $(\sqrt{s}=7 \mathrm{TeV})$. Backgrounds not listed have been omitted as there were zero events after the event selection. The electron $p_{\mathrm{T}}$ cut is on the reconstructed electrons.

previous sentences, and the ATLAS default electron $p_{\mathrm{T}}$ cut of $20 \mathrm{GeV}$. The effect of increasing the $p_{\mathrm{T}}$ cut for a specific trigger is to reduce the number of events, however the ratio of the number of signal to background events improves. For an electron with a $p_{\mathrm{T}}$ cut of $20 \mathrm{GeV}$, requiring the dilepton trigger, rather than a single lepton trigger, only slightly decreases the number of signal events, while maintaining the same signalto-background ratio. A breakdown of the true dilepton channels contributing to the 112.1 signal events using the EF_e10_medium trigger with an electron $p_{\mathrm{T}}$ cut of 20 $\mathrm{GeV}$ is shown below:

$$
e e=98.5 \quad e \mu=0.4 \quad e \tau=12.9 \quad \tau \tau=0.3
$$

Most of the dilepton events are from the ee channel. In the et channel a leptonic decay of the $\tau$ lepton into an electron is also considered a signal. The small contribution from the $e \mu$ channel likely comes from a jet reconstructed as a fake electron. 
For the $\mu \mu$ channel the two triggers studied are EF_mu10 and EF_2mu4, with two different reconstructed muon $p_{\mathrm{T}}$ cuts for each. The breakdown of the signal and background event numbers are shown in Table 4.16. The event selection essentially removes all $W \rightarrow l \nu+$ jet events, differing from the electron case likely because the purity of the muon reconstruction is near $100 \%$, so there are few jets that will fake a muon. Despite the fact there is a larger invariant mass cut around the $Z$ mass window, there are more $Z \rightarrow l l+$ jets events passing the $\mu \mu$ channel selection compared to the $e e$ channel for single lepton triggers. The true dilepton event types contributing to the $120.2 t \bar{t}$ dilepton events with EF_mu10 (with a $p_{\mathrm{T}}$ cut of $20 \mathrm{GeV}$ ) are:

$$
\mu \mu=105.7 \quad \mu \tau=14.1 \quad \tau \tau=0.4 .
$$

Again, there is a large fraction of events passing the selection from the $\tau$ decays in the $\mu \tau$ channel. For the a muon with a $p_{\mathrm{T}}$ cut of $20 \mathrm{GeV}$, requiring a dilepton trigger significantly reduces the number of signal events. This is due to the inefficiency of the muon trigger. For a single muon trigger in the $\mu \mu$ channel there are two muons in the event that can initiate the trigger, increasing the overall likelihood the event will be selected.

The $e \mu$ channel has both an electron and muon in the final state. The triggers considered for this channel are called the EFOR and EFAND triggers. The EFOR requires either the EF_e10_medium or the EF_mu10 trigger to pass. The EFAND trigger requires both the EF_e5_medium and the EF_mu4 triggers to pass (this combination trigger does not currently exist in the ATLAS trigger menu). Since the event rate for dilepton trigger is less, the minimum $p_{\mathrm{T}}$ of the electrons and muons for this trigger can be low. The breakdown of the signal and background events for these triggers is shown in Table 4.17 for the $e \mu$ channel selection. For the EFOR trigger two 


\begin{tabular}{|r||c|c|c|c|}
\hline & $\begin{array}{c}\text { EF_mu10 } \\
\left(p_{\mathrm{T}}>15 \mathrm{GeV}\right)\end{array}$ & $\begin{array}{c}\text { EF_mu10 } \\
\left(p_{\mathrm{T}}>20 \mathrm{GeV}\right)\end{array}$ & $\begin{array}{c}\text { EF_2mu4 } \\
\left(p_{\mathrm{T}}>10 \mathrm{GeV}\right)\end{array}$ & $\begin{array}{c}\text { EF_2mu4 } \\
\left(p_{\mathrm{T}}>20 \mathrm{GeV}\right)\end{array}$ \\
\hline \hline$t \bar{t} \rightarrow$ dilepton & 148.5 & 120.2 & 127.1 & 87.6 \\
\hline$t \bar{t} \rightarrow l$ jet & 1.2 & 0.3 & 3.0 & 0.2 \\
$W \rightarrow l \nu+$ jets & 0.0 & 0.0 & 2.6 & 0.0 \\
$Z \rightarrow l l+$ jets & 42.4 & 33.9 & 37.0 & 21.8 \\
Dibosons & 5.8 & 4.6 & 5.2 & 3.5 \\
Single top & 7.5 & 5.3 & 6.9 & 4.2 \\
\hline signal $(S)$ & $148.5 \pm 1.8$ & $120.2 \pm 1.7$ & $127.1 \pm 1.7$ & $87.6 \pm 1.4$ \\
background $(B)$ & $56.9 \pm 5.1$ & $44.1 \pm 4.6$ & $54.7 \pm 4.9$ & $29.7 \pm 3.7$ \\
$S / B$ & 2.61 & 2.73 & 2.32 & 2.95 \\
$S / \sqrt{S+B}$ & 10.4 & 9.4 & 9.4 & 8.1 \\
\hline \hline
\end{tabular}

Table 4.16: Signal and background number of events after the $\mu \mu$ event selection for 200 $\mathrm{pb}^{-1}(\sqrt{s}=7 \mathrm{TeV})$. Backgrounds not listed have been omitted as there were zero events after the event selection. The muon $p_{\mathrm{T}}$ cut is on the reconstructed muons.

$p_{\mathrm{T}}$ cuts on the reconstructed leptons are shown, $15 \mathrm{GeV}$ and $20 \mathrm{GeV}$. The EFAND trigger also has two $p_{\mathrm{T}}$ cuts shown, $10 \mathrm{GeV}$ and $20 \mathrm{GeV}$. The $e \mu$ channel has the highest signal-to-background ratio of all three dilepton channels. The $Z \rightarrow l l+$ jets background is reduced, while the single top background increases. The breakdown of the true dilepton events in the EFOR trigger selection (using lepton $p_{\mathrm{T}}$ cuts of 20 $\mathrm{GeV})$ is:

$$
e e=0.0 \quad \mu \mu=0.3 \quad e \mu=265.6 \quad e \tau=17.6 \quad \mu \tau=15.9 \quad \tau \tau=0.9 .
$$

In general for the three channels, the application of a reconstructed lepton $p_{\mathrm{T}}$ cut of $20 \mathrm{GeV}$ does the best to improve the signal to background ratio, rather than using a lower $p_{\mathrm{T}}$ value closer to the edge of the trigger turn-on region. However, the lower trigger $p_{\mathrm{T}}$ does gain statistics for the signal and background events (which might be of interest with small amounts of ATLAS data at the beginning). The dilepton and 


\begin{tabular}{|r||c|c|c|c|}
\hline & $\begin{array}{c}\text { EFOR } \\
\left(p_{\mathrm{T}}>15 \mathrm{GeV}\right)\end{array}$ & $\begin{array}{c}\text { EFOR } \\
\left(p_{\mathrm{T}}>20 \mathrm{GeV}\right)\end{array}$ & $\begin{array}{c}\text { EFAND } \\
\left(p_{\mathrm{T}}>10 \mathrm{GeV}\right)\end{array}$ & $\begin{array}{c}\text { EFAND } \\
\left(p_{\mathrm{T}}>20 \mathrm{GeV}\right)\end{array}$ \\
\hline \hline$t \bar{t} \rightarrow$ dilepton & 361.5 & 300.3 & 348.4 & 252.5 \\
\hline$t \bar{t} \rightarrow l$ ljet & 10.6 & 5.2 & 19.6 & 4.03 \\
$W \rightarrow l \nu+$ jets & 15.7 & 6.9 & 25.5 & 5.4 \\
$Z \rightarrow l l+$ jets & 33.7 & 13.6 & 55.7 & 12.1 \\
Dibosons & 14.6 & 12.2 & 14.4 & 10.5 \\
Single top & 23.8 & 19.1 & 24.0 & 16.3 \\
\hline signal $(S)$ & $361.5 \pm 2.9$ & $300.3 \pm 2.6$ & $348.4 \pm 2.8$ & $252.5 \pm 2.4$ \\
background $(B)$ & $98.3 \pm 6.3$ & $57.1 \pm 4.6$ & $139.3 \pm 7.5$ & $48.3 \pm 4.3$ \\
$S / B$ & 3.68 & 5.26 & 2.51 & 5.23 \\
$S / \sqrt{S+B}$ & 16.9 & 15.9 & 15.8 & 14.6 \\
\hline \hline
\end{tabular}

Table 4.17: Signal and background number of events afterthe $e \mu$ event selection for 200 $\mathrm{pb}^{-1}(\sqrt{s}=7 \mathrm{TeV})$. Backgrounds not listed have been omitted as there were zero events after the event selection. EFOR means either EF_e10_medium or EF_mu10 must pass, and EFAND means both of the EF_e5_medium and EF_mu4 triggers must pass in the event. The lepton $p_{\mathrm{T}}$ cut is on the reconstructed electrons and muons.

combination triggers with the lepton $p_{\mathrm{T}}$ cuts of $20 \mathrm{GeV}$ have a comparable signal-tobackground compared to the single lepton case. These triggers will be a very good option when the LHC is running at higher instantaneous luminosity and many of the single lower $p_{\mathrm{T}}$ lepton triggers considered here will need to be prescaled or tightened due to their large rates.

\subsection{Cross-section Calculation}

In particle physics a cross-section $\sigma$ represents the probability of interaction between partons to produce a defined final outcome. It is quoted in units of area reflecting the effective surface of interaction between the partons. There are several ingredients 
used to calculate the cross-section:

$$
\sigma=\frac{S}{\epsilon \int \mathcal{L} d t}
$$

$S$ is the number of observed signal events (without backgrounds) after all selection and trigger requirements, $\epsilon$ is the efficiency of the selection processes with respect to the true signal, and $\int \mathcal{L} d t$ is the integrated luminosity. Each of these variables are discussed in more detail below. In this analysis $\epsilon$ will be split into two pieces:

$$
\epsilon=\epsilon_{R} \times \epsilon_{T}
$$

where $\epsilon_{R}$ is the reconstruction efficiency with respect to the truth, taking into account detector acceptance losses. And $\epsilon_{T}$ is the trigger efficiency with respect to the reconstruction.

When doing an analysis with data (versus MC) the number of observed 'signal' events has contributions from both the true signal and backgrounds. To find the observed number of signal events one would subtract the expected number of background events, using MC studies or other measurements from data to calculate the expected number of background events in the final sample. In this MC analysis, $S$ is taken to be the final number of signal events after all reconstruction and trigger selections.

\subsubsection{Reconstruction Efficiency}

The reconstruction efficiency was calculated using the MC, and is one overall number calculated as:

$$
\epsilon_{R}=\frac{\text { number of reconstructed events }}{\text { number of true signal events }}
$$




\begin{tabular}{|c||c|c|c|}
\hline & $e e$ & $\mu \mu$ & $e \mu$ \\
\hline \hline$\epsilon_{R}[\%]$ & $5.18 \pm 0.07$ & $5.76 \pm 0.07$ & $13.90 \pm 0.1$ \\
\hline \hline
\end{tabular}

Table 4.18: Reconstruction efficiencies with respect to the true dilepton channel for the $e e, \mu \mu$, and $e \mu$ channels.

the number of reconstructed events is calculated after all selections except for the application of the trigger criteria. The number of true signal events are the number of dilepton events with electrons and muons in the final state (including those that come from leptonic $\tau$ decays).

For each of the three dilepton signal channels the channel reconstruction efficiency is listed in Table 4.18. The reconstruction efficiency for the ee and $\mu \mu$ channel is similar, with differences arising because the $\mu \mu$ channel has a larger cut on the invariant mass of the two muons, and because electrons and muons have slightly different object reconstruction efficiencies. For the $e \mu$ channel the reconstruction efficiency is the greatest because the event selection cuts are relaxed, with a lower missing transverse energy cut and no cut on the invariant mass of the two leptons.

The largest source of systematic uncertainty for reconstruction and identification of the signal channel comes from the jet energy scale uncertainty. Jets are made of electromagnetic and hadronic components. As was discussed in Chapter 3 hadron showers are complicated by the many interactions that are possible, and require calibration to account for invisible energy deposition that is not reconstructed by the calorimeter. Reconstructed jets are then a composition of not only different hadrons, but also electromagnetic objects. In the first year of ATLAS data taking the energy resolution for jets is expected to be $\pm 10 \%$ [33]. For electrons the energy resolution is expected to be $\pm(1-2) \%$, and for muons the transverse momentum resolution will be $\pm 3 \%$ [33] (for the type of reconstructed objects considered in this analysis). There 
will also be an uncertainty from the missing transverse energy calculation, which is directly correlated to the energy resolution of the other objects in the event.

In the analysis a cut was made on the number of jets and the jet $p_{\mathrm{T}}$, and so these large uncertainties on the jet reconstructed energy can influence the number of observed signal events. The effect of jet energy scale uncertainty was studied by varying each element of the jet 4 -vector $\left(E, p_{x}, p_{y}, p_{z}\right)$ by $\pm 5 \%$ (optimistic) and $\pm 10 \%$ (realistic), and propagating this change into the missing transverse energy calculation. Table 4.19 shows the relative change in the $t \bar{t}$ cross-section for each signal channel under these variations. Even an uncertainty of $\pm 5 \%$ on the jet energy scale translates into a variation in the measured cross-section by about $1.5 \%$. The realistic scenario with $200 \mathrm{pb}^{-1}$ of data is a jet energy uncertainty of $\pm 10 \%$, and this uncertainty alone causes a variation greater than $3 \%$ on the measured $t \bar{t}$ cross-section.

A check was also made on the effect of varying the jet reconstruction algorithm, changing the choice from the default AntiKt4Topo to AntiKt4Tower, Cone4Tower, or Cone4Topo causes only a small change in the measured cross-section $(<1.0 \%)$. The other two jet reconstruction algorithms, Cone7Tower and AntiKt6Topo, create jets in the event that are quite different from the nominal; they do cause slightly larger variation in the cross-section, but are not considered a major source of systematic uncertainty with this analysis. The variation due to the jet algorithm is negligible compared to the jet energy scale uncertainty.

\subsubsection{Trigger Efficiency}

In Section 4.6 it was shown that the single lepton and dilepton triggers had similar signal-to-background ratios when a $p_{\mathrm{T}}$ cut of $20 \mathrm{GeV}$ was applied to the lepton trigger object. The single lepton triggers do yield a higher number of signal events, and are 


\begin{tabular}{|l|c|c|}
\hline Jet 4-Vector Variation & Channel & $\Delta \sigma_{t \bar{t}} / \sigma_{t \bar{t}}[\%]$ \\
\hline \hline \multirow{3}{*}{$-5 \%$} & $e e$ & -2.0 \\
& $\mu \mu$ & -1.5 \\
& $e \mu$ & -1.5 \\
\hline \multirow{3}{*}{$+5 \%$} & $e e$ & 1.6 \\
& $\mu \mu$ & 2.0 \\
\hline \multirow{3}{*}{$10 \%$} & $e \mu$ & 1.3 \\
& $e e$ & -3.2 \\
& $\mu \mu$ & -3.6 \\
$+10 \%$ & $e \mu$ & -3.2 \\
\hline \hline
\end{tabular}

Table 4.19: The relative difference in the in the $t \bar{t}$ cross-section measurement with respect to the default when the jet energy 4 -vector is changed by $\pm 5 \%$ and $\pm 10 \%$.

likely a better choice for the trigger while low $p_{\mathrm{T}}$ trigger thresholds are available (at low LHC luminosity). Section 4.5.6 showed the electron and muon trigger efficiencies for the single lepton triggers. It was shown that once an appropriate $p_{\mathrm{T}}$ cut was applied to reconstructed electrons that was above the trigger turn-on region, then the electron trigger efficiency was approximately flat for all electrons. For muons it was shown that the trigger efficiency did strongly depend on the muon $\eta$ and $\phi$. To account for these dependences a two-dimensional histogram was generated in $\eta-\phi$ space for the electron and muon trigger efficiency, and are shown Figure 4.27.

This section discusses the application of the object trigger efficiencies for the single lepton triggers in the cross-section measurement. The $t \bar{t}$ signal channel and trigger 


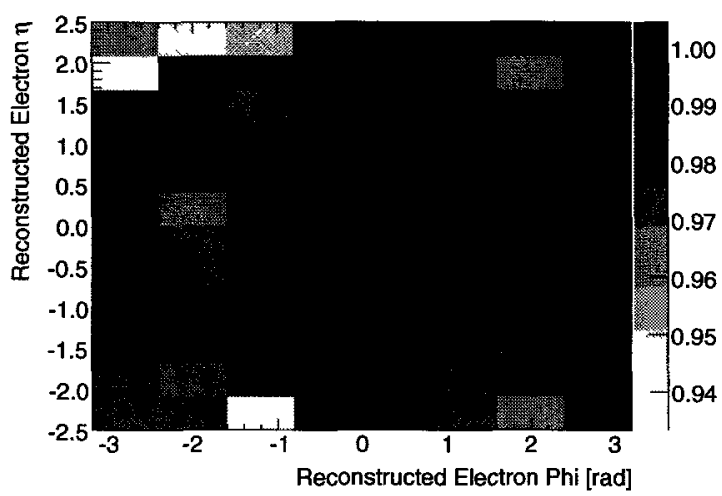

(a) EF_e10_medium

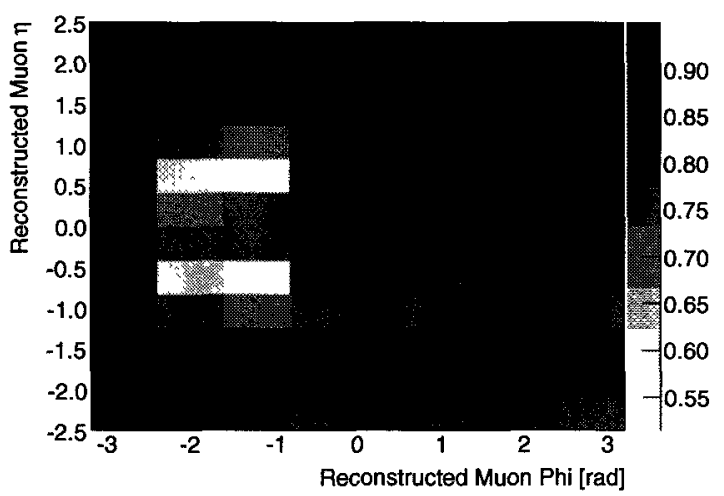

(b) EF_mu10

Figure 4.27: Two-dimensional trigger efficiency for EF_e10_medium and EF_mu10 in $\eta-\phi$.

chosen for each channel are:

$$
\begin{aligned}
e e & : \text { EF_e10_medium } \\
\mu \mu & : \text { EF_mu10 } \\
e \mu & : \text { EF_mu10 or EF_e10_medium }
\end{aligned}
$$

In all cases the reconstructed electrons and muons are required to have $p_{\mathrm{T}}>20 \mathrm{GeV}$. To account for variations in the trigger efficiency with geometric location the crosssection in Equation 4.8 is modified to included event-by-event trigger efficiencies:

$$
\sigma=\frac{S}{\epsilon \int \mathcal{L} d t} \rightarrow \frac{S}{\epsilon_{R} \epsilon_{T} \int \mathcal{L} d t}
$$

where:

$$
\epsilon_{T}=\frac{\sum_{i=1}^{n_{r}} \epsilon_{T, i}}{n_{r}}
$$

$\epsilon_{T, i}$ is the trigger efficiency for event $i .^{6}$ The sum $i$ is over all events that pass the reconstruction requirements for that channel $n_{r}$ (note that the trigger condi-

\footnotetext{
${ }^{6}$ The MC@NLO event weights have also been taken into account, event by event.
} 


\begin{tabular}{|c||c|c|c|}
\hline & $e e$ & $\mu \mu$ & $e \mu$ \\
\hline \hline$\epsilon_{T}[\%]$ & 99.9 & 96.7 & 99.8 \\
\hline \hline
\end{tabular}

Table 4.20: Triggers efficiencies (with respect to the reconstruction) for the $e e, \mu \mu$, and $e \mu$ channels. The statistical errors are on the order of $10^{-2} \%$.

tion is not required here). The event trigger efficiency is calculated by summing over all reconstructed objects of that trigger type in the event, so all electrons for EF_e10_medium, all muons for EF_mu10, and all electrons and muons when either EF_mu10 or EF_e10_medium are required:

$$
\epsilon_{T, \imath}=\left(1-\prod_{o=1}^{n_{o}}\left(1-\epsilon_{T, o}\right)\right)_{\imath}
$$

The efficiency for the $o^{\text {th }}$ reconstructed object $\epsilon_{T, o}$ is drawn from the 2D $\eta-\phi$ trigger efficiency map, generated as described in Section 4.5.6. For example, if the analysis uses the EF_mu10 trigger and there is only one reconstructed muon in the event then the trigger efficiency for the event is $\epsilon_{T, \imath}=\epsilon_{T, 0}$. For more than one muon in the event the trigger efficiency for that event increases.

The final trigger efficiencies $\epsilon_{T}$ used in the cross-section measurement for each of the channels is shown in Table 4.20 .

In ATLAS the object trigger efficiency maps for electrons and muons will be extracted from samples of $Z \rightarrow e e$ and $Z \rightarrow \mu \mu$ using one lepton as a tag and the other as a probe to determine the efficiency. A process like $Z \rightarrow l l$ is likely to be used to extract the trigger efficiency as it has a clear signal and large cross-section (with respect to $t \bar{t}$ ) and two leptons of the same kind. The user would need to ensure that any differences in the trigger efficiency between the $Z$ and $t \bar{t}$ samples be accounted for in the application of one efficiency to the other. The value for $\epsilon_{T}$ in the cross- 
section Equation 4.10 would be calculated as described above using MC samples (to determine the efficiency of the trigger with respect to the reconstruction), only using the real object trigger efficiency in $\eta-\phi$ space (instead of MC generated efficiency maps as has been done here).

The systematic uncertainties that were investigated for the trigger efficiency were carried out by generating and applying the $\eta-\phi$ trigger efficiency maps from the $Z$ samples, and by changing the $\Delta R$ matching criteria between the trigger and reconstructed objects from 0.1 to 0.05 and 0.15 . Taking into account these variations the trigger efficiency for the ee channel did not change, however for the $\mu \mu$ and $e \mu$ channels the systematic uncertainty from trigger efficiencies was found to change the $t \bar{t}$ cross-section by $\pm 0.7 \%$ and $\pm 0.9 \%$, respectively.

\subsubsection{Luminosity and Associated Systematic Uncertainty}

The luminosity $(\mathcal{L})$ is an extremely important parameter for any cross-section measurement, as it is a measure of the rate of interactions between the colliding protons. Since the field of particle physics deals with physics at an extremely small scale (the scale of fundamental particles) in practice this area is quoted in units of barn, where 1 barn $=10^{-28} \mathrm{~m}^{2}$.

The instantaneous luminosity is given by:

$$
\mathcal{L}=\frac{\mu n_{b} f_{r}}{\epsilon_{\mathcal{L}} \sigma_{\text {inel }}},
$$

and is calculated using the number of bunch crossings at the interaction point $\left(n_{b}\right)$ in one accelerator revolution period $\left(1 / f_{r}\right)$, from calculations or measurements of the average number of observed interactions per bunch crossing $(\mu)$, and the efficiency $\left(\epsilon_{\mathcal{L}}\right)$ of the detector and algorithm used to measure the total inelastic cross-section 
$\left(\sigma_{\text {inel }}\right)$. Known inputs from the accelerator settings are $n_{b}$ (which has a maximum value of 2808 bunch crossings) and $f_{r}$ that has a value of $11 \mathrm{kHz}$ in the LHC. The denominator $\epsilon_{\mathcal{L}} \sigma_{\text {inel }}$ is discussed in more detail later, but can be calculated using either MC predictions or from accelerator parameter information.

In ATLAS there are several detectors available to independently measure $\mu$, and hence the luminosity. The following is a description of some of the detectors used for luminosity measurements in ATLAS:

Minimum Bias Trigger Scintillator (MBTS) : This detector is composed of scintillator paddles ( $2 \mathrm{~cm}$ thick) arranged in disks perpendicular to the beam direction. There are two detector arrays located on either side of the IP just before the endcap calorimeter cryostat (at $z= \pm 3.56 \mathrm{~cm}$ ) labelled A side and C side. The primary goal of the MBTS is to trigger on minimum collision activity in the forward region $(2.04<|\eta|<3.84)$. A hit in the MBTS is defined as having a signal above some predefined threshold corresponding to a single particle. For the luminosity measurement the trigger is required to coincide in time with bunches that are configured to collide in ATLAS, and can require a single hit on one or both side $\mathrm{A}$ and $\mathrm{C}$. To remove contamination from backgrounds, the difference in time between the recorded hits in the MBTS on side $\mathrm{A}$ and $\mathrm{C}$ must be less than $10 \mathrm{~ns}$ to be consistent with particles originating from the IP.

\section{Luminosity measurement using Čerenkov Integrating Detector (LUCID)}

: The primary goal of LUCID is to provide to ATLAS with online and offline luminosity measurement. Located at $z= \pm 17 \mathrm{~m}$, these two Čerenkov detector modules are composed of $161.5 \mathrm{~m}$ long tubes, each with a $15 \mathrm{~mm}$ diameter, near the beam pipe, pointing to the IP (at $|\eta|=5.8$ ). The Čerenkov light measured 
in a gas mixture is collected by photomultiplier tubes (PMTs). The amplitude of the PMT signal is proportional to the number of particles entering the tube.

Liquid Argon Endcaps : Calorimeter channels with significant energy in the EMEC inner wheel $(2.5<|\eta|<3.2)$ and FCal $(3.1<|\eta|<4.9)$ are used to determine events with significant energy depositions. A channel with significant energy is required to have energy greater than five times the expected electronic noise, and have energy greater than $250 \mathrm{MeV}(1200 \mathrm{MeV})$ for the EMEC (FCal). For an event to be used in the luminosity calculation it first must pass a MBTS trigger, and there must be at least two channels with significant energy on both side $\mathrm{A}$ and $\mathrm{C}$. The average time is calculated for each side using all channels with significant energy. The final criteria on a good luminosity event is that the difference in time between side $\mathrm{A}$ and $\mathrm{C}$ be less than $5 \mathrm{~ns}$.

Inner Detector : The inner detector is used to count the rate of events the pass certain track requirements. One method counts the number of events that have two or more tracks reconstructed with the inner detector with $p_{\mathrm{T}}>100 \mathrm{MeV}$. These tracks must be consistent with coming from the primary vertex and meet a minimum number of hits in each of the inner detector layers.

For each detector the basic idea is that the event rate (accounting for efficiencies, acceptance) is proportional to the average number of interactions per bunch crossing. There are additional corrections made to account for non-linearities between the event rate and $\mu$. The backgrounds to the detectors from sources such as beam halo or beam gas interactions, are determined by studying the rates for LHC bunch crossings that are not configured for collisions.

The following describes two methods of calculating the luminosity that have been used in ATLAS for the data taking period of 2010. The first method calculates the 


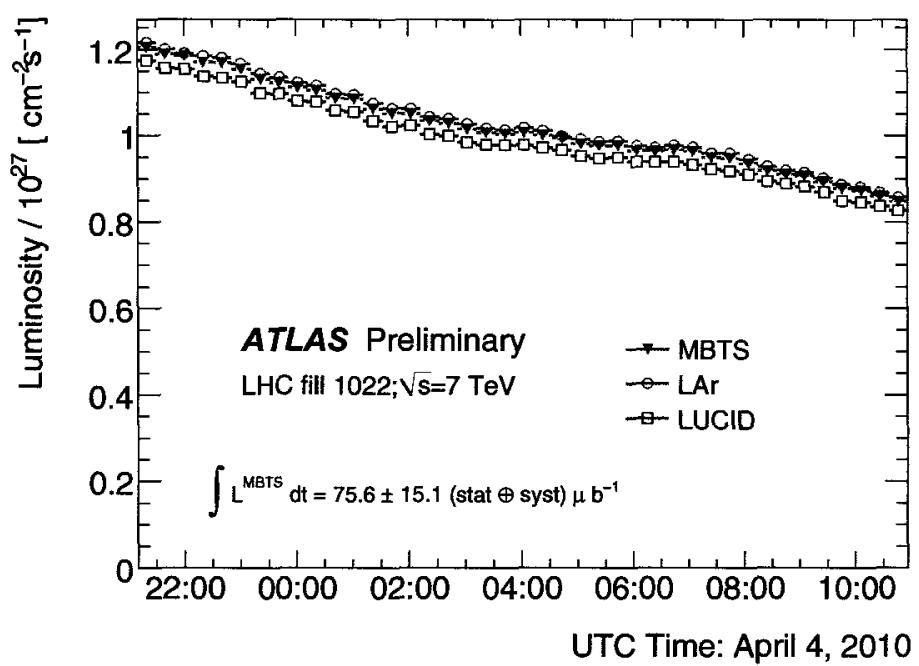

Figure 4.28: Instantaneous luminosity as a function of time using three ATLAS luminosity detectors, with normalization to Pythia MC predictions, during a run on April 4, 2010. This figure has been taken from [66].

denominator in Equation 4.12 from the inelastic cross-section and associated efficiency for a specific detector and algorithm. Unfortunately the inelastic cross-section for $p p$ collisions at $7 \mathrm{TeV}$ has not as yet been measured so at this time the value must come from MC predictions, and there are large uncertainties associated to the $\mathrm{MC}$ predictions stemming from large generator differences in the cross-sections, particle multiplicities and kinematics. It is unknown if the full envelope of values from the various $\mathrm{MC}$ generators even encompasses the true value. To account for these uncertainties, the denominator of Equation 4.12 using $\mathrm{MC}$ predictions is assigned a systematic error of $20 \%$. Figure 4.28 shows an example of the instantaneous luminosity calculation as a function of time using the $\mathrm{MC}$ predicted normalization, for MBTS, LUCID, and the liquid argon endcaps with ATLAS data taken on April 4, 2010. In this plot statistical errors are too small to be seen, and the systematic error is independent of time. The different methods track each other well.

A second method for calculating the normalization of the luminosity comes from 
measurements of the accelerator properties. It is called van der Meer scan [67]. One beam is deflected in the horizontal or vertical plane with respect to the other beam, and the count rate as a function of net displacement in that plane is measured with the luminosity detectors. Each of the distributions in the horizontal $(R(x))$ and vertical planes $(R(y))$ is bell-shape, with the peak centered at zero corresponding to head-on collisions between both beams. For this method the luminosity is calculated as:

$$
\mathcal{L}=\frac{n_{b} f_{r} I_{1} I_{2}}{2 \pi \Sigma_{x} \Sigma_{y}}
$$

using the same $n_{b}$ and $f_{r}$ from Equation 4.12 , and the number of particles per bunch in beam 1 and $2\left(I_{1}\right.$ and $\left.I_{2}\right)$, and where $\Sigma_{x}$ is defined as:

$$
\Sigma_{x}=\frac{\int R(x) d x}{\sqrt{2 \pi} R_{x}(0)}
$$

$R_{x}(0)$ is the rate at zero displacement (the peak height). The same definitions apply for $\Sigma_{y}$ in the vertical plane. The theory assumes the particle densities in $x$ and $y$ are independent. In ATLAS three scans were performed in the spring of 2010 in both the horizontal and vertical planes to extract the luminosity using this method. It was found $R(x)$ and $R(y)$ were best fit to double Gaussians with a flat background (measured from rates of non-colliding bunches). The luminosity measurement was consistent for the different luminosity detectors and algorithms. Calculation of the luminosity using this van der Meer normalization yields a systematic uncertainty of $11 \%$, with the largest source of systematic uncertainty on the luminosity measurement coming from the intensity of the beams [66].

Luminosity is measured instantaneously and then integrated over time. The product of the integrated luminosity and the cross-section for process $A$ gives the expected 
number of events from $A$ in time $d t$ : Num evts $\mathrm{A}=\int \mathcal{L} d t \times \sigma_{A}$

For this thesis it is assumed that the uncertainty on the integrated luminosity is $10 \%$. This is a conservative number, given that this analysis assumes $200 \mathrm{pb}^{-1}$ of integrated luminosity, and an uncertainty of $11 \%$ using the van der Meer method is limited to information from three luminosity scans, and with $\sim 3 \mathrm{pb}^{-1}$ delivered to the experiment.

\subsubsection{Variation with Monte Carlo Generator}

The choice of generator slightly affects the kinematics of objects in top events (recall Section 4.2.2), which can result in variations of the total number of observed events. The different generators also have slightly different branching ratios for the $W$ leptonic decays, therefore slightly different cross-sections for the dilepton channel. For example, in MC@NLO (with a branching ratio of $W \rightarrow l \nu_{l}=11.1 \%$ ) the branching ratio for $t \bar{t} \rightarrow(W b)(W b) \rightarrow\left(e \nu_{e} b\right)\left(e \nu_{e} b\right)=(0.111)(0.111)=1.23 \%$, where as for POWHEG (with a branching ratio of $W \rightarrow l \nu_{l}=10.8 \%$ ) the branching ratio for the same $t \bar{t}$ decay would be $1.17 \%$. The Acer LO MC generator is not considered here, as it is known to have an imprecise calculation of the cross-section and very different kinematics from the NLO generators that better represent the current understanding of the $t \bar{t}$ production at $7 \mathrm{TeV}$. The final systematic uncertainty associated with the choice of generator for each of the channels is taken to be:

$$
e e: \Delta \sigma_{t \bar{t}} / \sigma_{t \bar{t}}= \pm 1.6 \% \quad \mu \mu: \Delta \sigma_{t \bar{t}} / \sigma_{t \bar{t}}= \pm 1.1 \% \quad \mu e: \Delta \sigma_{t \bar{t}} / \sigma_{t \bar{t}}= \pm 1.6 \%
$$

and is the difference of the measured cross-section using the POWHEG samples with respect to the cross-section obtained using the default MC@NLO sample. 


\begin{tabular}{|l|c|c|c|}
\hline & $e e$ & $\mu \mu$ & $e \mu$ \\
\hline \hline reconstruction efficiency & $\pm 3.2 \%$ & $+3.3 \%,-3.6 \%$ & $+2.5 \%,-3.2 \%$ \\
trigger efficiency & $\pm 0.0 \%$ & $\pm 0.7 \%$ & $\pm 0.9 \%$ \\
generator choice & $\pm 1.6 \%$ & $\pm 1.1 \%$ & $\pm 1.6 \%$ \\
luminosity & $\pm 10.0 \%$ & $\pm 10.0 \%$ & $\pm 10.0 \%$ \\
theoretical uncertainty & $\pm 6.0 \%$ & $\pm 6.0 \%$ & $\pm 6.0 \%$ \\
\hline total & $\pm 12.2 \%$ & $+12.2 \%,-12.3 \%$ & $+12.1 \%,-12.2 \%$ \\
\hline \hline
\end{tabular}

Table 4.21: Summary of the systematic uncertainties to the top pair cross-section measurement, $\Delta \sigma / \sigma$.

\subsubsection{Summary of Uncertainties}

The systematic uncertainties considered in this analysis are the uncertainty associated to the reconstruction and trigger efficiency, generator choice, and luminosity. An additional systematic is assigned from the theoretical calculation, based on [49], to which the samples were scaled with the $k$-factors. Based on the uncertainty in that same reference, the theoretical uncertainty in the calculation of the $t \bar{t}$ cross section is taken to be $\pm 6 \%$. In the paper the absolute uncertainty is quoted with respect to $\sigma_{t \bar{t}}$ at the LHC with $\sqrt{s}=14 \mathrm{TeV}$, but the same relative uncertainty has been assigned here for $\sigma_{t \bar{t}}$ at $\sqrt{s}=7 \mathrm{TeV}$.

Table 4.21 breaks down the systematic uncertainties on the cross-section measurement. The error due to the reconstruction efficiency is taken solely as the variation of the jet energy scale by $\pm 10 \%$, which is significantly larger than any other variations in the reconstruction algorithms. The total systematic uncertainty is the sum of each added in quadrature.

This analysis uses only MC generated events. A cross section measurement using data requires subtraction of the expected number of background events to find $S$. This estimation can be made using data driven measurements or can be based on MC 
predictions. Since the number of background events are not subtracted in the analysis, the uncertainty associated with the cross-section for each of these backgrounds has not been discussed.

If only the statistics for $200 \mathrm{pb}^{-1}$ were considered, then the statistical error on each channel would be:

$$
\begin{array}{r}
e e: \sqrt{112} \rightarrow \Delta \sigma_{t \bar{t}} / \sigma_{t \bar{t}}= \pm 9.5 \% \\
\mu \mu: \sqrt{120} \rightarrow \Delta \sigma_{t \bar{t}} / \sigma_{t \bar{t}}= \pm 9.1 \% \\
e \mu: \sqrt{300} \rightarrow \Delta \sigma_{t \bar{t}} / \sigma_{t \bar{t}}= \pm 5.8 \%
\end{array}
$$

However, combining the results of the three dilepton channels will reduce the overall statistical error on the measurement.

\subsubsection{Comparison of the Cross-section Calculation}

The MC@NLO sample has been used to study the $t \bar{t}$ cross-section in the dilepton channel. There are two independent MC@NLO samples that cover the complete phase space of SM $t \bar{t}$ decays, one containing the semileptonic and dileptonic decays, and the other containing the fully hadronic channel. Combining the cross-sections of these samples the total $t \bar{t}$ cross-section as predicted by MC@NLO (for $m_{t}=172.5$ $\mathrm{GeV}$ ) is:

$$
\begin{array}{rcccc}
\text { (no } k \text {-factor) } & \frac{\sigma_{\text {semi+dilep }}[\mathrm{pb}]}{80.201} & \frac{\sigma_{\text {fully hadronic }}[\mathrm{pb}]}{64.046} & \frac{\sigma_{t \bar{t}}[\mathrm{pb}]}{144.247} \\
(k \text {-factor }) & 87.419 & & 73.653 & 161.072
\end{array}
$$

The fraction of the total cross-section that is due to the semileptonic and dileptonic 
$t \bar{t}$ decays is $55.60 \%$ (and $54.27 \%$ accounting for the $k$-factor).

Using the formalism developed in this thesis and Equation 4.10, the $t \bar{t}$ crosssections for each of the three signal channels were calculated and the following numbers obtained:

$$
\begin{aligned}
& e e: 161.0 \pm 15.3 \text { (stat) } \pm 19.6 \text { (sys) } \mathrm{pb} \\
& \mu \mu: 160.4 \pm 14.6 \text { (stat) } \pm 19.6 \text { (sys) pb } \\
& e \mu: 161.0 \pm 9.3 \text { (stat) } \pm 19.6 \text { (sys) pb }
\end{aligned}
$$

These agree within the statistical (stat) and systematic (sys) uncertainties to the generated MC@NLO input value of $161.072 \mathrm{pb}$. The statistical error assumes only $200 \mathrm{pb}^{-1}$ of data has been studied, and does not use the full MC statistics scaled to that luminosity (which would yield a negligible statistical error).

The current best theoretical prediction for the $t t$ cross-section is $\sigma_{t \bar{t}}=160.79 \pm 9.64$ $\mathrm{pb}$ [49]. This is very close to the number that is given by the generator used in this study (MC@NLO).

\subsubsection{Summary and Outlook}

An investigation of the top pair cross section measurement using the dilepton channel with the ATLAS detector has been carried out using MC datasets, for $\sqrt{s}=7 \mathrm{TeV}$ with $200 \mathrm{pb}^{-1}$ of data. The largest source of systematic uncertainty is from the determination of the luminosity. This measurement is expected to improve from $11 \%$ to $4-5 \%$ by the end of 2011 . A large statistical error will be present with only 200 $\mathrm{pb}^{-1}$ of data, but combining the dilepton channels with each other, and possibly other $t \bar{t}$ channels will reduce the error.

The $e \mu$ channel is the golden channel for the dilepton search. It has a branching 
ratio two times that of the $e e$ and $e \mu$ channel, and suffers significantly less background (mostly due to the large $Z$ boson background in the other two channels). As a result, this channel has the highest reconstruction efficiency, and has a signal-to-background ratio of nearly twice that of the other channels. This gives further motivation to include a specific 'emu' trigger, requiring both an electron and a muon in the ATLAS trigger menu ${ }^{7}$.

As the instantaneous luminosity of the detector increases towards $10^{32} \mathrm{~cm}^{-2} \mathrm{~s}^{-1}$ (which may be achieved by early 2011) and eventually $10^{34} \mathrm{~cm}^{-2} \mathrm{~s}^{-1}$ (design luminosity) the trigger rates for the low energy and momentum threshold triggers will increase. The low threshold single lepton triggers considered in this analysis are EF_e10_medium and EF_mu10, and are the default triggers used for the ATLAS top cross-section measurement with early ATLAS data. At higher luminosities, these triggers are expected to be either prescaled or removed from the ATLAS trigger menu, in favour of lower rate triggers with higher thresholds such as EF_e20_medium and EF mu20. Moving to a higher threshold in the trigger implies that a higher cut will need to be applied to the reconstructed lepton $p_{\mathrm{T}}$ to avoid biases from the trigger turn-on region. The dilepton triggers presented here (EF_2e5_medium, EF_mu10, EF_e5_medium AND EF_mu6) have low threshold cuts, but they also have a low trigger rate, and even at higher luminosity they are not expected to be prescaled or modified. Using these triggers means that the same $p_{\mathrm{T}}$ cut of $20 \mathrm{GeV}$ can be applied to electrons and muons, as has been done in this analysis.

\footnotetext{
${ }^{7}$ Such as EF_e5_medium and EF_mu4.
} 


\section{Chapter 5}

\section{Conclusion}

The field of particle physics is one that has grown over the decades with collaboration of people all over the world. Theorists work to develop theories and push the precision of their predictions. The boundaries of technology are pushed to create more powerful accelerators and precise detectors to test these theories. Scientific experimentalists analyse and understand the output from the machines to search for signatures of old and new physics, that then feed back to the theoretical community. This cycle has been ongoing for decades, and with the LHC era pushing experimental data to new energy regimes, this cycle is expected to continue for many more decades to come.

The work in this thesis has presented in detail some of these elements. Every piece of the ATLAS detector must be studied separately, and then as a combined system to take advantage of the full potential of this giant detector. In this thesis, a detailed study of the ATLAS FCal was done using test beam data and MC studies. This thesis also studied the potential to make a top pair cross-section measurement in the ATLAS detector within the first year of data taking. This measurement involves the reconstruction of electrons, muons, jets, and missing transverse energy, and so it requires all subsystems of the ATLAS detector be understood and operational. 


\section{Appendix A}

\section{Monte Carlo Data Set Information for Top Physics}

Tables A1 to A6 provide additional information about the samples used in the $t \bar{t}$ crosssection analysis. The sample identification numbers are those used by the ATLAS collaboration. The ATLAS reconstruction tag associated to all of the samples is r1302.

\begin{tabular}{|l|c|c|c|c|}
\hline Process & Sample ID & Generator + Parton Shower & $\sigma[\mathrm{pb}]$ & $k$-factor \\
\hline \hline$t \bar{t} \rightarrow\left(l \nu, q q^{\prime}\right),(l \nu, l \nu)$ & 5200 & MC@NLO + HERWIG/JIMMY & 80.201 & 1.09 \\
\hline$t \bar{t} \rightarrow\left(l \nu, q q^{\prime}\right),(l \nu, l \nu)$ & 5860 & POWHEG + HERWIG & 79.118 & 1.10 \\
$t \bar{t} \rightarrow\left(l \nu, q q^{\prime}\right),(l \nu, l \nu)$ & 5861 & POWHEG + PYTHIA & 79.118 & 1.10 \\
$t \bar{t} \rightarrow\left(l \nu, q q^{\prime}\right),(l \nu, l \nu)$ & 5205 & AcerMC + HERWIG/JIMMY & 57.98 & 1.50 \\
\hline \hline
\end{tabular}

Table A.1: Additional Monte Carlo sample information for the $t \bar{t}$ samples. Cross-sections are for $p p$ collisions at $7 \mathrm{TeV}$. The $k$-factor is the ratio of the expected cross-section at NNLO calculated in [49] to that of the matrix element generator. 


\begin{tabular}{|c|c|c|c|c|}
\hline Process & Sample ID & Generator + Parton Shower & $\sigma[\mathrm{pb}]$ & $k$-factor \\
\hline$Z \rightarrow e e \mathrm{~Np} 0$ & 107650 & ALPGEN + HERWIG/JIMMY & 661.9 & 1.22 \\
\hline$Z \rightarrow e e \mathrm{~Np} 1$ & 107651 & ALPGEN + HERWIG/JIMMY & 133.3 & 1.22 \\
\hline$Z \rightarrow e e \mathrm{~Np} 2$ & 107652 & ALPGEN + HERWIG/JIMMY & 40.3 & 1.22 \\
\hline$Z \rightarrow e e \mathrm{~Np} 3$ & 107653 & ALPGEN + HERWIG/JIMMY & 11.2 & 1.22 \\
\hline$Z \rightarrow e e \mathrm{~Np} 4$ & 107654 & ALPGEN + HERWIG/JIMMY & 2.7 & 1.22 \\
\hline$Z \rightarrow e e \mathrm{~Np} 5$ & 107655 & ALPGEN + HERWIG/JIMMY & 0.8 & 1.22 \\
\hline$Z \rightarrow \mu \mu \mathrm{Np} 0$ & 107660 & ALPGEN + HERWIG/JIMMY & 657.7 & 1.22 \\
\hline$Z \rightarrow \mu \mu \mathrm{Np} 1$ & 107661 & ALPGEN + HERWIG/JIMMY & 132.8 & 1.22 \\
\hline$Z \rightarrow \mu \mu \mathrm{Np} 2$ & 107662 & ALPGEN + HERWIG/JIMMY & 39.6 & 1.22 \\
\hline$Z \rightarrow \mu \mu \mathrm{Np} 3$ & 107663 & ALPGEN + HERWIG/JIMMY & 11.1 & 1.22 \\
\hline$Z \rightarrow \mu \mu \mathrm{Np} 4$ & 107664 & ALPGEN + HERWIG/JIMMY & 2.8 & 1.22 \\
\hline$Z \rightarrow \mu \mu \mathrm{Np} 5$ & 107665 & ALPGEN + HERWIG/JIMMY & 0.8 & 1.22 \\
\hline$Z \rightarrow \tau \tau \mathrm{Np} 0$ & 107670 & ALPGEN + HERWIG/JIMMY & 657.4 & 1.22 \\
\hline$Z \rightarrow \tau \tau \mathrm{Np} 1$ & 107671 & ALPGEN + HERWIG/JIMMY & 133.0 & 1.22 \\
\hline$Z \rightarrow \tau \tau \mathrm{Np} 2$ & 107672 & ALPGEN + HERWIG/JIMMY & 40.4 & 1.22 \\
\hline$Z \rightarrow \tau \tau \mathrm{Np} 3$ & 107673 & ALPGEN + HERWIG/JIMMY & 11.0 & 1.22 \\
\hline$Z \rightarrow \tau \tau \mathrm{Np} 4$ & 107674 & ALPGEN + HERWIG/JIMMY & 2.9 & 1.22 \\
\hline$Z \rightarrow \tau \tau \mathrm{Np} 5$ & 107675 & ALPGEN + HERWIG/JIMMY & 0.7 & 1.22 \\
\hline
\end{tabular}

Table A.2: Z backgrounds. Additional Monte Carlo sample information for the $Z \rightarrow l l$ samples. Cross-sections are for $p p$ collisions at $7 \mathrm{TeV}$. The $k$-factor corrects the crosssections to NNLO precision [33]. Np stands for number of partons. The samples include the full Drell-Yan contributions. 


\begin{tabular}{|c|c|c|c|c|}
\hline Process & Sample ID & Generator + Parton Shower & $\sigma[\mathrm{pb}]$ & $k$-factor \\
\hline$W \rightarrow e \nu \mathrm{Np} 0$ & 107680 & ALPGEN + HERWIG/JIMMY & 6913.3 & 1.22 \\
\hline$W \rightarrow e \nu \mathrm{Np} 1$ & 107681 & ALPGEN + HERWIG/JIMMY & 1293.0 & 1.22 \\
\hline$W \rightarrow e \nu \mathrm{Np} 2$ & 107682 & ALPGEN + HERWIG/JIMMY & 377.1 & 1.22 \\
\hline$W \rightarrow e \nu \mathrm{Np} 3$ & 107683 & ALPGEN + HERWIG/JIMMY & 100.9 & 1.22 \\
\hline$W \rightarrow e \nu \mathrm{Np} 4$ & 107684 & ALPGEN + HERWIG/JIMMY & 25.3 & 1.22 \\
\hline$W \rightarrow e \nu \mathrm{Np} 5$ & 107685 & ALPGEN + HERWIG/JIMMY & 6.9 & 1.22 \\
\hline$W \rightarrow \mu \nu \mathrm{Np} 0$ & 107690 & ALPGEN + HERWIG/JIMMY & 6935.4 & 1.22 \\
\hline$W \rightarrow \mu \nu \mathrm{Np} 1$ & 107691 & ALPGEN + HERWIG/JIMMY & 1281.2 & 1.22 \\
\hline$W \rightarrow \mu \nu \mathrm{Np} 2$ & 107692 & ALPGEN + HERWIG/JIMMY & 375.3 & 1.22 \\
\hline$W \rightarrow \mu \nu \mathrm{Np} 3$ & 107693 & ALPGEN + HERWIG/JIMMY & 101.1 & 1.22 \\
\hline$W \rightarrow \mu \nu \mathrm{Np} 4$ & 107694 & ALPGEN + HERWIG/JIMMY & 25.7 & 1.22 \\
\hline$W \rightarrow \mu \nu \mathrm{Np} 5$ & 107695 & ALPGEN + HERWIG/JIMMY & 7.0 & 1.22 \\
\hline$W \rightarrow \tau \nu \mathrm{Np} 0$ & 107700 & ALPGEN + HERWIG/JIMMY & 6835.8 & 1.22 \\
\hline$W \rightarrow \tau \nu \mathrm{Np} 1$ & 107701 & ALPGEN + HERWIG/JIMMY & 1276.8 & 1.22 \\
\hline$W \rightarrow \tau \nu \mathrm{Np} 2$ & 107702 & ALPGEN + HERWIG/JIMMY & 376.6 & 1.22 \\
\hline$W \rightarrow \tau \nu \mathrm{Np} 3$ & 107703 & ALPGEN + HERWIG/JIMMY & 100.8 & 1.22 \\
\hline$W \rightarrow \tau \nu \mathrm{Np} 4$ & 107704 & ALPGEN + HERWIG/JIMMY & 25.7 & 1.22 \\
\hline$W \rightarrow \tau \nu \mathrm{Np} 5$ & 107705 & ALPGEN + HERWIG/JIMMY & 7.0 & 1.22 \\
\hline
\end{tabular}

Table A.3: W backgrounds. Additional Monte Carlo sample information for the $W \rightarrow l \nu$ samples. Cross-sections are for $p p$ collisions at $7 \mathrm{TeV}$. The $k$-factor corrects the crosssections to NNLO precision [33]. Np stands for number of partons. 


\begin{tabular}{|l|c|c|c|c|}
\hline Process & Sample ID & Generator + Parton Shower & $\sigma[\mathrm{pb}]$ & $k$-factor \\
\hline \hline$W W \rightarrow(l \nu)(l \nu) \mathrm{Np} 0$ & 107100 & ALPGEN + HERWIG/JIMMY & 3.23 & 1.21 \\
$W W \rightarrow(l \nu)(l \nu) \mathrm{Np} 1$ & 107101 & ALPGEN + HERWIG/JIMMY & 1.71 & 1.21 \\
$W W \rightarrow(l \nu)(l \nu) \mathrm{Np} 2$ & 107102 & ALPGEN + HERWIG/JIMMY & 0.87 & 1.21 \\
$W W \rightarrow(l \nu)(l \nu) \mathrm{Np} 3$ & 107103 & ALPGEN + HERWIG/JIMMY & 0.36 & 1.21 \\
$W Z \rightarrow($ incl $)(l l) \mathrm{Np} 0$ & 107104 & ALPGEN + HERWIG/JIMMY & 1.05 & 1.21 \\
$W Z \rightarrow($ incl $)(l l) \mathrm{Np} 1$ & 107105 & ALPGEN + HERWIG/JIMMY & 0.73 & 1.21 \\
$W Z \rightarrow(\mathrm{incl})(l l) \mathrm{Np} 2$ & 107106 & ALPGEN + HERWIG/JIMMY & 0.43 & 1.21 \\
$W Z \rightarrow(\mathrm{incl})(l l) \mathrm{Np} 3$ & 107107 & ALPGEN + HERWIG/JIMMY & 0.20 & 1.21 \\
$Z Z \rightarrow(\mathrm{incl})(l l) \mathrm{Np} 0$ & 107108 & ALPGEN + HERWIG/JIMMY & 0.80 & 1.21 \\
$Z Z \rightarrow(\mathrm{incl})(l l) \mathrm{Np} 1$ & 107109 & ALPGEN + HERWIG/JIMMY & 0.39 & 1.21 \\
$Z Z \rightarrow(\mathrm{incl})(l l) \mathrm{Np} 2$ & 107110 & ALPGEN + HERWIG/JIMMY & 0.18 & 1.21 \\
$Z Z \rightarrow(\mathrm{incl})(l l) \mathrm{Np} 3$ & 107111 & ALPGEN + HERWIG/JIMMY & 0.06 & 1.21 \\
\hline \hline
\end{tabular}

Table A.4: Diboson backgrounds. Additional Monte Carlo sample information for the diboson $(W W, Z Z, W Z)$ samples. Cross-sections are for $p p$ collisions at $7 \mathrm{TeV}$. The $k$-factor corrects the cross-sections to NNLO precision [33]. Np stands for number of partons.

\begin{tabular}{|l|c|c|c|c|}
\hline Process & Sample ID & Generator + Parton Shower & $\sigma$ [pb] & $k$-factor \\
\hline \hline$t \bar{t} \rightarrow\left(q q^{\prime}\right)\left(q q^{\prime}\right)$ & 105204 & MC@NLO + HERWIG/JIMMY & 64.046 & 1.15 \\
single top $W t$-channel & 108346 & MC@NLO + HERWIG/JIMMY & 14.581 & 1.0 \\
single top $t$-channel $(e$ channel) & 108340 & MC@NLO + HERWIG/JIMMY & 7.152 & 1.0 \\
single top $t$-channel $(\mu$ channel) & 108341 & MC@NLO + HERWIG/JIMMY & 7.176 & 1.0 \\
single top $t$-channel $(\tau$ channel) & 108342 & MC@NLO + HERWIG/JIMMY & 7.128 & 1.0 \\
single top $s$-channel $(e$ channel) & 108343 & MC@NLO + HERWIG/JIMMY & 0.4685 & 1.0 \\
single top $s$-channel $(\mu$ channel) & 108344 & MC@NLO + HERWIG/JIMMY & 0.4684 & 1.0 \\
single top $s$-channel $(\tau$ channel) & 108345 & MC@NLO + HERWIG/JIMMY & 0.4700 & 1.0 \\
\hline \hline
\end{tabular}

Table A.5: Top backgrounds. Additional Monte Carlo sample information for the single top and all hadronic $t \bar{t}$ samples. Cross-sections are for $p p$ collisions at $7 \mathrm{TeV}$. The $k$-factor corrects the cross-sections to NNLO precision [33]. 


\begin{tabular}{|c|c|c|c|c|}
\hline Process & Sample ID & Generator + Parton Shower & $\sigma[\mathrm{pb}]$ & $k$-factor \\
\hline \hline QCD J0 & 105009 & PYTHIA & 97529070000 & - \\
QCD J1 & 105010 & PYTHIA & 673020000 & - \\
QCD J2 & 105011 & PYTHIA & 41194700 & - \\
QCD J3 & 105012 & PYTHIA & 2193250 & - \\
QCD J4 & 105013 & PYTHIA & 87848.7 & - \\
QCD J5 & 105014 & PYTHIA & 2328.56 & - \\
QCD J6 & 105015 & PYTHIA & 33.8461 & - \\
\hline \hline
\end{tabular}

Table A.6: QCD di-jet backgrounds. Additional Monte Carlo sample information for the QCD samples. Cross-sections are for $p p$ collisions at $7 \mathrm{TeV}$. There is no $k$-factor quoted here as it has not been calculated for QCD. The different QCD J $x$ samples were generated for a different $p_{\mathrm{T}}$ ranges, J0: 8-17 GeV, J1: $17-35 \mathrm{GeV}$, J2: 35-70 GeV, J3: 70-140 GeV, J4: $140-280 \mathrm{GeV}, \mathrm{J} 5: 280-560 \mathrm{GeV}, \mathrm{J} 6: 560+\mathrm{GeV}$. They must be scaled independently and then added together to get the correct spectrum. 


\section{Appendix B}

\section{Efficiency Uncertainty}

This appendix describes techniques which are commonly used to calculate errors associated with an efficiency measurement. Throughout this chapter the efficiency is defined as:

$$
\epsilon=\frac{k}{n},
$$

where $k$ is the number of events selected out of $n$ total chances. The following description can be applied to total event statistics or individual bins in a distribution.

\section{B.1 The Standard Methods}

In this section it is assumed that each event is unweighted, the treatment of weighted events is presented in Section B.2

\section{B.1.1 Binomial Statistics}

The quantity $k$ is binomially distributed, events either pass or fail some criteria. The probability density function for the Binomially distributed variable $k$ given some true 
efficiency $\epsilon$ and total number of events $n$ is:

$$
P(k ; n, \epsilon)=\frac{n !}{k !(n-k) !} \epsilon^{k}(1-\epsilon)^{n-k} .
$$

The expectation value and variance for this discrete variable $k$ are:

$$
\begin{aligned}
& E(k)=\hat{k}=n \epsilon \\
& V(k)=\sigma_{k}=E\left(k^{2}\right)-[E(k)]^{2}=n \epsilon(1-\epsilon) .
\end{aligned}
$$

To calculate the error associated with the efficiency defined in Equation B.1, the total number of events $n$ are assumed to be known with certainty $\left(\sigma_{n}=0\right)$. By propagation of error the variance associated with the efficiency is:

$$
V(\epsilon)=\sigma_{\epsilon}^{2}=\frac{\epsilon}{n}(1-\epsilon) .
$$

One issue with this result is that the value of the true efficiency is required in evaluating the variance of the efficiency, which is ultimately the quantity being calculated. Also, if the efficiency is either zero or one then the variance is zero, regardless of the statistics.

\section{B.1.2 Poisson Statistics}

Poisson statistics are the limiting case of the Binomial distribution when $n \rightarrow \infty$ and $P(k ; n, \epsilon) \rightarrow 0$ such that the total efficiency remains the same. The probability density function describing the outcome $k$ given $n$ trials and some efficiency $\epsilon$ is:

$$
P(k ; n, \epsilon)=\frac{\lambda^{k} e^{-\lambda}}{k !}
$$


where $\lambda=n \epsilon$. The expectation value and variance of $k$ are:

$$
E(k)=\lambda \quad V(k)=\lambda .
$$

Poisson statistics are often used to quote errors associated to histogram bins, making use of the expectation value and variance for a Poisson distribution by citing bin contents as

$$
k \pm \sqrt{k} \text { and } n \pm \sqrt{n}
$$

(bin indices have been neglected throughout). If the efficiency is defined as in Equation B.1 and propagation of errors performed on the two (assumed independent) variables with Poisson errors as defined in Equation B.3, then the variance on the efficiency would be:

$$
V(\epsilon)=\sigma_{\hat{\epsilon}}^{2}=\epsilon^{2}\left(\frac{1}{k}+\frac{1}{n}\right)
$$

This technique has the same flaws as the Binomial technique, in that when $\epsilon=0$ the variance is zero, and the true efficiency is used to evaluate the variance of the estimated efficiency.

\section{B.1.3 Bayesian Statistics}

The goal of this chapter is to evaluate the efficiency of some measurement and make some quantitative statement reflecting the certainty of the efficiency given the measurements of $k$ and $n$. Using Bayesian statistics the probability for an efficiency given measurements of $k$ and $n$ can be written as:

$$
P(\epsilon ; k, n)=\frac{P(k ; n, \epsilon)}{P(\epsilon ; n)} C
$$


where $P(k ; n, \epsilon)$ is the Binomial probability density for $k$, as was previously defined in Equation B.2. $P(\epsilon ; n)$ encodes any prior knowledge of $\epsilon$. The efficiency value will be constrained to lie between zero and one, and has equal probability for any of these values in between before any measurement is taken. $P(\epsilon ; n)$ is therefore taken to be a uniform distribution in the range of zero and one. The variable $C$ is the overall normalization constant to ensure the integral over all space of the probability density function is equal to one, and is $C=1 /(n+1)[68,69]$. By substituting these variables into the Bayesian formula the probability to measure an efficiency $\epsilon$ is given by:

$$
P(\epsilon ; k, n)=\frac{n !}{k !(n-k) !} \epsilon^{k}(1-\epsilon)^{n-k}(n+1)
$$

in the range $[0,1]$, and zero outside of this range.

The expectation value of $\epsilon$ and mode (where $d P / d \epsilon=0$ is the mode) of this distribution are calculated to be:

$$
\begin{aligned}
E(\epsilon) & =\hat{\epsilon}=\frac{k+1}{n+2} \\
\text { mode } & =\frac{k}{n} .
\end{aligned}
$$

Using this technique it is not the mean that returns the familiar form of the efficiency, but rather the mode. For large values of $k$ and $n$ they are approximately equivalent. The variance of the efficiency is:

$$
V(\epsilon)=\frac{(k+1)(k+2)}{(n+2)(n+3)}-\frac{(k+1)^{2}}{(n+2)^{2}} .
$$

In this analysis the value of the efficiency quoted is the most probable value, and asymmetric errors are taken about the mode that span the region of $\pm \sigma$. This 
technique removes the problems associated with the Binomial and Poisson method, however it does create a small bias in using the mode for the calculated efficiency instead of the mean. Notice that even if $n=0$ the mean value of the distribution will be equal to $1 / 2$ and the variance will be $1 / 12$, which are the mean and variance of a uniform distribution in the range $[0,1]$. For $n=0$ the efficiency as defined using the mode has no meaning and no value will be quoted for the efficiency or its uncertainty.

\section{B.2 Weighted Events}

The Bayesian method for calculating the errors associated with an efficiency method suffers the problem that for certain values of $k$ greater than $n$ (which can happen with negatively weighted events) the variance can be negative. There is a ROOT function that will calculate Bayesian errors for the user, enclosing $68.3 \%$ of the distribution (TGraphAsymmErrors: : BayesDivide [9]), however it can not be used if the entries are weighted. This is a problem for the $t \bar{t}$ analysis as approximately $15 \%$ of the MC@NLO $t \bar{t}$ events have a negative weight. Currently there is no suggested prescription in ATLAS for properly handling this problem. If one simply uses the sum of the weights for $k$ and $n$ then evaluation of the statistical error will not properly take into account the full statistics of the sample. For example, if Poisson statistics are assumed, if 1000 events are sampled but the sum of weights is 800 then the quoted value should be $800 \pm \sqrt{1000}($ not $800 \pm \sqrt{800})$. Despite this problem this is the method taken in this analysis, and the values of $k$ and $n$ used to calculate the statistical error in Equation B.6 are the sum of the weights. 


\begin{tabular}{|c|c|c|c|c|c|c|}
\hline & \multicolumn{2}{|c|}{ Poisson } & \multicolumn{2}{c|}{ Binomial } & \multicolumn{2}{c|}{ Bayesian } \\
& No EW & EW & No EW & EW & No EW & EW \\
\hline \hline$k$ & 1600 & 1218 & 1600 & 1218 & 1600 & 1218 \\
$n$ & 1719 & 1301 & 1719 & 1301 & 1719 & 1301 \\
$\epsilon$ & 0.9308 & 0.9362 & 0.9308 & 0.9362 & 0.9308 & 0.9362 \\
\hline$\sigma_{\epsilon}$ & \pm 0.0323 & \pm 0.0373 & \pm 0.0061 & \pm 0.0068 & ${ }_{-0.0066}^{+0.0056}$ & ${ }_{-0.0075}^{+0.0001}$ \\
\hline \hline
\end{tabular}

Table B.1: Example of different ways to calculate the error on an efficiency calculation. $\mathrm{EW}=$ event weight taken into account.

\section{B.3 Example}

An example of the overall efficiency as calculated using Poisson, Binomial, and Bayesian statistics is shown in Table B.1. In the table the acronym EW stands for event weight, so 'No EW' implies that the event weight is not taken into account. When the event weight is not taken into account, the full statistics are used to calculate the error, so for a given statistics method the overall error is smaller. The Poisson and Binomial errors are symmetric. The Bayesian errors are symmetric about the expectation value of the distribution, but the mode is used to quote the efficiency, and asymmetric errors are taken about this average.

The three methods of calculating the error on the efficiency are also applied to individual bins. An example of a histogram using these errors on the individual bins is shown in Figure B.1. The error bars using Poisson statistics are significantly larger, and incorrect for reasons previously mentioned. The errors calculated using the Binomial and Bayesian statistics appear similar in many cases. However, in this example there are a few bins where the efficiency is $100 \%(\eta \sim-1.7,+1)$; for these cases the Binomial error on that bin is zero, whereas for the Bayesian statistics there is a non-zero value for the lower error (since the prior of the distribution states the value of the efficiency can not be greater than one). 


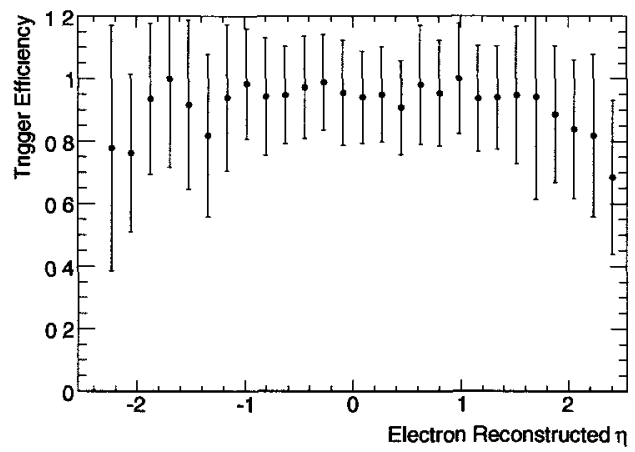

(a) Poisson statistics

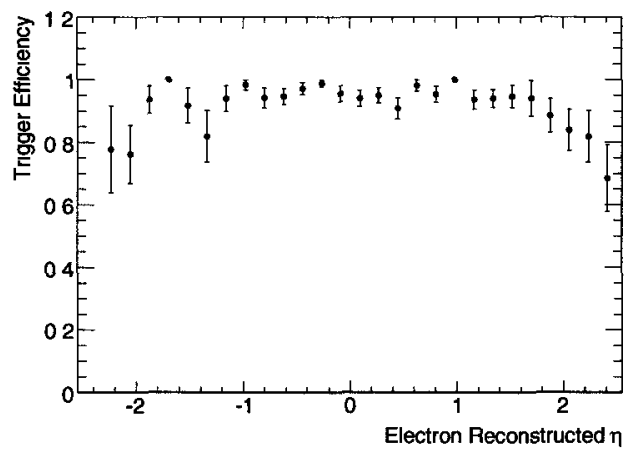

(b) Binomial statistics

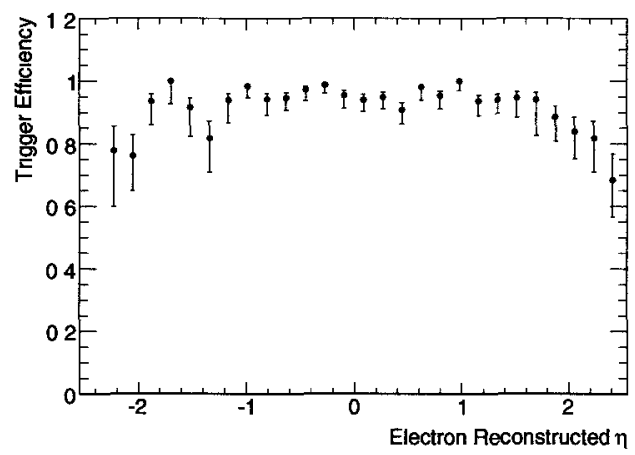

(c) Bayesian statistics

Figure B.1: An example trigger efficiency distribution when the error on the efficiency is calculated using (a) Poisson (b) Binomial and (c) Bayesian statistics. 


\section{References}

[1] K. Nakamura et al., Review of Particle Physics, J. Phys. G 37, 075021 (2010).

[2] S. L. Glashow, Partial-Symmetries of Weak Interactions, Nucl. Phys. 22, 579 (1961).

[3] S. Weinberg, A Model of Leptons, Phys. Rev. Lett. 19, 1264 (1967).

[4] A. Salam, Weak and electromagnetic interactions, in Proceedings in Elementary Particle Theory (The Nobel Symposium), p. 367, Lerum, Sweden, 1968.

[5] R. Fernow, Introduction to Experimental Particle Physics, Cambridge University Press (1986).

[6] CDF and D0 Collaborations, Combination of Tevatron searches for the standard model Higgs boson in the $W^{+} W^{-}$decay mode, Phys. Rev. Lett. 104, 061802 (2010).

[7] ATLAS Collaboration, The ATLAS Experiment at the CERN Large Hadron Collider, JINST 3, S08003 (2008).

[8] ATLAS Collaboration, ATLAS Computing: Technical Design Report, ATLASTDR-017, CERN-LHCC-2005-022 (2005).

[9] R. Brun and F. Rademakers, ROOT An Object Oriented Data Analysis Framework, Nucl. Inst. and Meth. A 389, 81 (1997), See also http://root.cern.ch/.

[10] Worldwide LHC Computing Grid, 2010, http://lcg.web.cern.ch/LCG/.

[11] T. Maeno, PanDA: distributed production and distributed analysis system for ATLAS, J. Phys.: Conf. Ser. 119, 062036 (2008). 
[12] M. Branco, Don Quijote - Data Management for the ATLAS Automatic Production System, in Computing in High Energy Physics and Nuclear Physics 2004, p. 661, Interlaken, Switzerland, 2004, CERN Document Server 865674.

[13] W. Leo, Techniques for Nuclear and Particle Physics Experiments, SpringerVerlag (1987).

[14] H. Messel and D. F. Crawford, Electron-photon Shower Distribution Function, Pergamon Press (1970).

[15] D. Perkins, Introduction to High Energy Physics, Cambridge University Press (2000).

[16] C. W. Fabjan and R. Wigmans, Energy measurement of elementary particles, Rep. Prog. Phys. 52, 1519 (1989).

[17] A. Artamonov et al., The ATLAS Forward Calorimeters, JINST 3, P02010 (2008).

[18] A. Savine et al., Position Measurements and Response Uniformity of the LAr Prototype for ATLAS, in Proceedings of the 7th International Conference on Calorimetry in High Energy Physics, p. 361, World Scientific, 1998.

[19] J. Archambault et al., Energy Calibration of the ATLAS Liquid Argon Forward Calorimeter, JINST 3, P02002 (2008).

[20] A. Savine, Private Communication, (2007).

[21] L. Nagel and D. Pederson, SPICE (Simulation Program with Integrated Circuit Emphasis), University of California, Berkley (1973).

[22] J. Rutherfoord, The Shape of the ATLAS Forward Calorimeter Current Pulse, ATLAS-LARG-PUB-2006-004 (2006).

[23] M. Schram, Performance of the Atlas Forward Calorimeter and search for the invisible Higgs via vector boson fusion at ATLAS, $\mathrm{PhD}$ thesis, Carleton University, 2008. 
[24] W. Cleland and E. Stern, Signal processing considerations for liquid ionization calorimeters in a high rate environment, Nucl. Instr. and Meth. A 338, 467 (1994).

[25] T. Virdee, Experimental Techniques, CERN-OPEN-2000-261 (1998).

[26] J. Armitage et al., Electron signals in the Forward Calorimeter prototype for ATLAS, JINST 2, P11001 (2007).

[27] J. Archambault, Private Communication, (2007).

[28] T. McCarthy, A Study of Hadronic Calibration Schemes for Pion Test Beam Data in the ATLAS Forward Calorimeter, Master's thesis, Carleton University, 2010.

[29] L. Heelan, Performance of the ATLAS forward calorimeter, J. Phys.: Conf. Ser. 160, 012058 (2008).

[30] ATLAS Collaboration, ATLAS Detector and Physics Technical Design Report, CERN/LHCC/99-14/15 (1999).

[31] T. Barillari et al., Local Hadronic Calibration, ATL-LARG-PUB-2009-001 (2009).

[32] E. Abat et al., Response and Shower Topology of 2 to $180 \mathrm{GeV}$ Pions Measured with the ATLAS Barrel Calorimeter at the CERN Test bam and Comparison to Monte Carlo Simulations, ATL-CAL-PUB-2010-001 (2010).

[33] ATLAS Collaboration, Expected performance of the ATLAS experiment: detector, trigger and physics, CERN-OPEN-2008-020 (2008).

[34] W. Lampl et al., Calorimeter Clustering Algorithms: Description and Performance, ATL-LARG-PUB-2008-002 (2008).

[35] GEANT4 Collaboration, Geant4 a simulation toolkit, Nucl. Inst. and Meth. A 506, 250 (2003).

[36] GEANT4 Collaboration: GEANT4 physics lists, http://www.geant4.org/ geant4/support/proc_mod_catalog/physics_lists/referencePL.shtml . 
[37] GEANT4 Collaboration, Geant4 physics lists: use cases: http://www.geant4. org/geant4/support/physicslists/referencepl/usecases.shtml.

[38] G. Folger and J. Wellisch, String parton models in Geant4, in Proceedings of International conference on Computing in High Energy and Nuclear Physics, La Jolla, California, 2003.

[39] H. W. Bertini, Low-Energy Intranuclear Cascade Calculation, Phys. Rev. 131, 1801 (1963).

[40] J. Birk, Theory of the Response of Organic Scintillation Crystals to Short-Range Particles, Phys. Rev. 86, 569 (1952).

[41] S. Amoruso et al., Study of electron combination in liquid argon with the ICARUS $T P C$, Nucl. Inst. and Meth. A 523, 275 (2004).

[42] J. Archambault, Monte Carlo studies of the Forward Calorimeter and of the Supersymmetric Top Quark, PhD thesis, Carleton University, 2009.

[43] CDF Collaboration, Observation of Top Quark Production in Pbar-P Collisions, Phys. Rev. Lett. 74, 2626 (1995).

[44] D0 Collaboration, Observation of the Top Quark, Phys. Rev. Lett. 74, 2632 (1995).

[45] N. Cabibbo, Unitary symmetry and Leptonic Decays, Phys. Rev. Lett. 10, 531 (1963).

[46] M. Kobayashi and T. Maskawa, CP Violation in the Renormalizable Theory of Weak Interaction, Prog. Theor. Phys. 49, 652 (1973).

[47] M. N. Z. Maki and S. Sakata, Remarks on the Unified Model of Elementary Particles, Prog. Theor. Phys. 28, 870 (1962).

[48] B. Pontecorvo, Neutrino experiments and the question of leptonic-charge conservation, Sov. Phys. JETP 26, 984 (1968).

[49] U. Langenfeld, S. Moch, and P. Uwer, Measuring the running top-quark mass, Phys. Rev. D 80, 054009 (2009). 
[50] The Tevatron Electroweak Working Group for the CDF and D0 Collaborations, Combination of CDF and DO Results on the Mass of the Top Quark, FERMILAB-TM-2427-E (2009).

[51] ALEPH, CDF, D0, DELPHI, L3, OPAL, SLD Collaborations, the LEP Electroweak Working Group, Tevatron Electroweak Working Group, SLD electroweak and heavy flavour groups, Precision Electroweak Measurements and Constraints on the Standard Model, CERN-PH-EP/2008-020 (2009).

[52] CDF Collaboration, Combination of CDF top quark pair production cross section measurements with up to $4.6 \mathrm{fb}^{-1}$, CDF Note 9913 (2009).

[53] S. Frixione and B.R.Webber, Matching NLO QCD Computations and Parton Shower Simulations, JHEP 06, 029 (2002).

[54] P. Nason, POWHEG [online], June 2010, Available from: http://mobydick.mib.infn.it/ nason/POWHEG/ .

[55] G. Corcella and all, HERWIG 6.5, JHEP 01, 010 (2001).

[56] J. Alwall et al., A standard format for Les Houches Event Files, Comput. Phys. Commun. 176, 300 (2007).

[57] A. Shibata et al., Understanding Monte Carlo Generators for Top Physics, ATLCOM-PHYS-2009-334 (2009).

[58] W. Lampl et al., Calorimeter Clustering Algorithms: Description and Performance, ATL-LARG-PUB-2008-0012 (2005).

[59] J. Hartert and I. Ludwig, Electron isolation in the ATLAS experiment, ATLCOM-PHYS-2010-070 (2010).

[60] MCTruthClassifier, https://twiki.cern.ch/twiki/bin/view/AtlasProtected/MCTruthClassifier 2010.

[61] ATLAS Collaboration, Search for top pair candidate events in ATLAS at $\sqrt{7}=$ $7 \mathrm{TeV}$, ATLAS-CONF-2010-063 (2010).

[62] S. Catani et al., Longitudinally-invariant $k_{\perp}$-clustering algorithms for hadron hadron collisions, Nucl. Phys. B 406, 187 (1993). 
[63] S. Ellis and D. Soper, Successive combination jet algorithm for hadron collisions, Phys. Rev. D 48, 3160 (1993).

[64] M. Cacciari et al., The anti-k $k_{t}$ jet clustering algorithm, JHEP 0804, 063 (2008).

[65] I. Abt et al., The tracking, calorimeter and muon detectors of the H1 experiment at HERA, Nucl. Inst. and Meth. A 386, 348 (1997).

[66] ATLAS Collaboration, Luminosity Determination Using the ATLAS Detector, ATL-CONF-2010-060 (2010).

[67] S. van der Meer, Calibration of the effective beam height in the ISR, CERN-ISRPO-68-31 (1968).

[68] T. Ullrich and Z. Xu, Treatment of Errors in Efficiency Calculation, arXiv:physics/0701199 (2007).

[69] M. Paterno, Calculating Efficiencies and Their Uncertainties, FERMILAB-TM2286-CD (2004). 